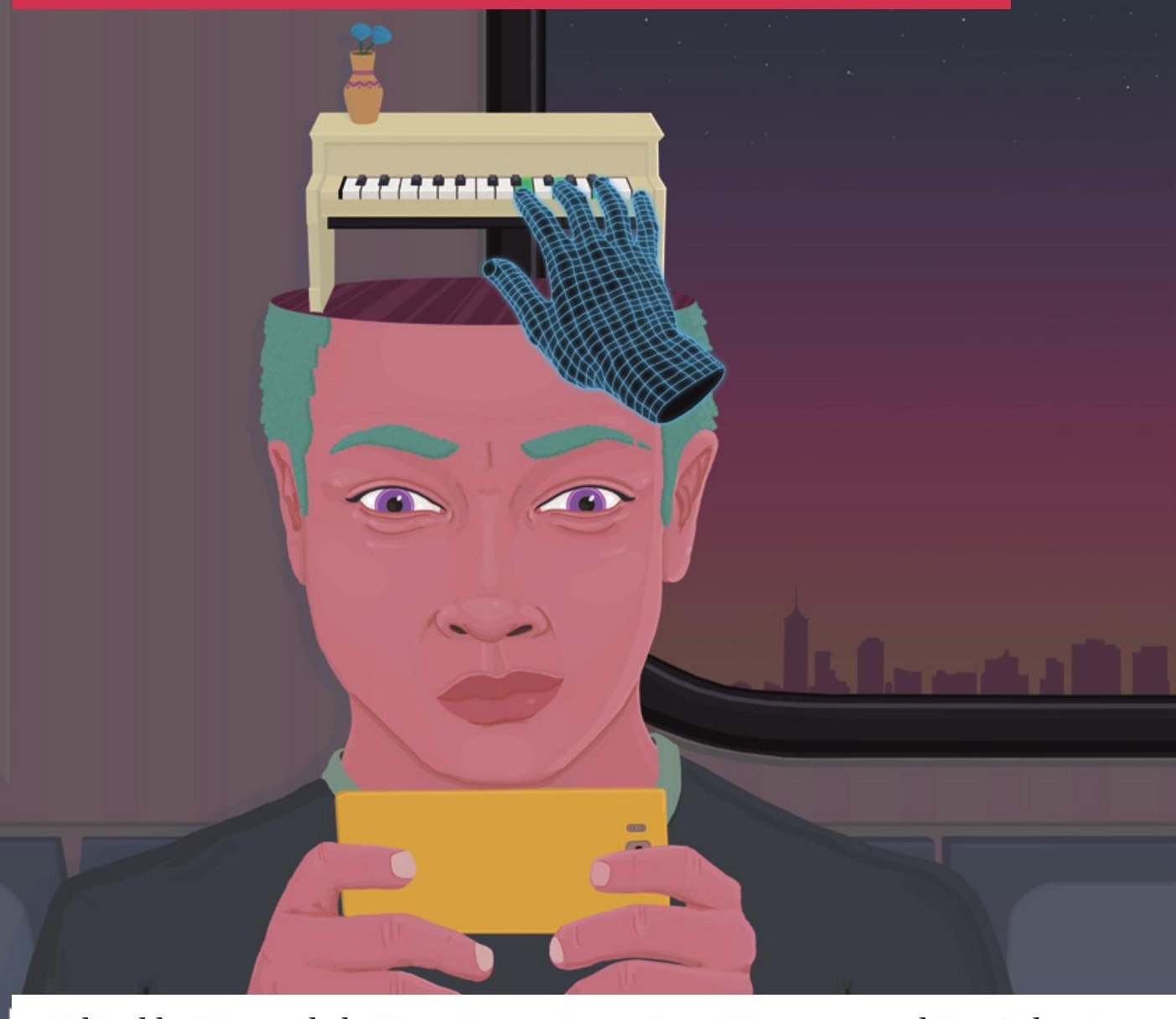

Edited by Teresa de la Hera, Jeroen Jansz, Joost Raessens and Ben Schouten

\title{
Persuasive Gaming in Context
}


Persuasive Gaming in Context 



\title{
Persuasive Gaming in Context
}

\author{
Edited by \\ Teresa de la Hera \\ Jeroen Jansz \\ Joost Raessens \\ Ben Schouten
}


This work is part of the research program 'Persuasive Gaming. From Theory-Based Design to Validation and Back' with project number 314-99-106 which is partly financed by the Netherlands Organization for Scientific Research (NWO).

Cover illustration: Miguel Méndez

Cover design: Coördesign, Leiden

Lay-out: Crius Group, Hulshout

$\begin{array}{ll}\text { ISBN } & 9789463728805 \\ \text { e-ISBN } & 9789048543939 \\ \text { DOI } & 10.5117 / 9789463728805 \\ \text { NUR } & 775 \mid 811\end{array}$

\section{(c) $\underset{\mathrm{BY}}{\mathrm{SC}} \Theta$}

Creative Commons License CC BY NC ND

(http://creativecommons.org/licenses/by-nc-nd/3.0)

@ T. de la Hera, J. Jansz, J. Raessens, B. Schouten / Amsterdam University Press B.V., Amsterdam 2021

Some rights reserved. Without limiting the rights under copyright reserved above, any part of this book may be reproduced, stored in or introduced into a retrieval system, or transmitted, in any form or by any means (electronic, mechanical, photocopying, recording or otherwise).

Every effort has been made to obtain permission to use all copyrighted illustrations reproduced in this book. Nonetheless, whosoever believes to have rights to this material is advised to contact the publisher. 


\section{Table of Contents}

1. Persuasive Gaming: From Theory-Based Design to Validation and Back. An Introduction

Teresa de la Hera, Jeroen Jansz, Ruud Jacobs, Ben Schouten, Joost

Raessens \& Martijn Kors

\section{Part I The Study of Persuasion Through Digital Games}

Introduction to Part I

Joost Raessens \& Teresa de la Hera

2. Persuasive Games, A Decade Later

Ian Bogost

3. Playing an Automated World

Miguel Sicart

4. Looking Beyond Persuasion Through Rule-Based

Representations in Digital Games: Designing Games to Shape,

Reinforce, or Change Attitudes

Teresa de la Hera \& Joost Raessens

5. Creating Stealth Game Interventions for Attitude and Behavior Change: An 'Embedded Design' Model

Geoff Kaufman, Mary Flanagan \& Max Seidman

\section{Part II Designing Persuasive Games}

Introduction to Part II

Ben Schouten \& Martijn Kors

6. A Breathtaking Journey. Appealing to Empathy in a Persuasive Mixed-Reality Game

Martijn Kors, Gabriele Ferri, Erik D. van der Spek, Cas Ketel, \& Ben Schouten 
7. Macro, Micro, and Meta-Persuasive Play to Change Society Lindsay D. Grace

8. VilDu?! A Game for Sexually Abused Children: How Openness Facilitated a Clear Design Direction

Menno Deen \& Eline Muijres

9. Designing for Persuasion through Embodied Experiences in Virtual Reality

SunJoo Ahn

\section{Part III Assessing the Effectiveness of Persuasive Games}

Introduction to Part III

Jeroen Jansz \& Ruud Jacobs

10. The Present of Persuasion: Escalating Research into Persuasive Game Effects

Ruud Jacobs \& Jeroen Jansz

11. Designing for Transfer

Mette Wichmand

12. Striving 'Against All Odds' to Reduce Prejudice toward Immigrants and Refugees

Chad Michael Wertley \& Jordan Soliz

13. Mitigating Bias and Improving Professional Decision-Making through Persuasive Training Games

Yu-Hao Lee, Norah E. Dunbar, Claude H. Miller, Elena Bessarabova, Matthew Jensen, Scott Wilson, Javier Elizondo, Judee Burgoon, \& Joseph Valacich 


\title{
1. Persuasive Gaming: From Theory- Based Design to Validation and Back. An Introduction
}

Teresa de la Hera, Jeroen Jansz, Ruud Jacobs, Ben Schouten, Joost Raessens \& Martijn Kors

\begin{abstract}
This chapter offers a multifaceted reflection on persuasive gaming divided into three pillars: persuasiveness, design, and validation. The first section on persuasiveness is a critical review of previous and current persuasive gaming theory and analysis. It argues that the contemporary gaming landscape needs to expand theoretically and presents a multidimensional persuasive approach as one way in which this can be done. The following section on the design of persuasive games looks at research on design principles, which are the defining characteristics of persuasive games. The final section on validation discusses existing studies on the effects of persuasive games and the case-based assessment of the impact of new games.
\end{abstract}

Keywords: procedural rhetoric; persuasive games; persuasive game design; persuasiveness; persuasive gaming effects

\section{Situating research on persuasive gaming}

The rapid developments in new communication technologies constantly create new opportunities in the media and entertainment industry. These developments have facilitated the popularization of digital games, which has translated into an exponential growth of the game industry in the last decades. Digital games have become part of our daily routines thanks to the ubiquitous presence of mobile devices, the simplification of game

Hera, T. Dela, J. Jansz, J. Raessens, B. Schouten, Persuasive Gaming in Context. Amsterdam: Amsterdam University Press, 2021 DOI 10.5117/9789463728805_CHO1 
interactions (for example through the use of touchscreens), and the diversification of genres that better respond to the different variety of expectations of the 2.6 billion players worldwide (Entertainment Software Association, 2018, p. 2). This is what game scholar Jesper Juul has dubbed the 'casual revolution', 'a breakthrough moment in the history of video games' (2010, p. 2). The ongoing ludification of culture has transformed the domain of play and games into something that is much more than a temporary and somewhat trivial escape from the seriousness of life (Raessens, 2014).

The ubiquitous presence of digital games has resulted in an expansion of the applications of these games from mere entertainment purposes to a great variety of serious purposes. Although Clark Abt published his book Serious Games already in 1970, it was only from the early years of the 21st century that research on the serious applications of digital games gained special relevance and attention from academics (Mateas \& Chen, 2006; Ritterfeld, Cody, \& Vorderer, 2009). Among serious games, defined as digital games used for purposes beyond mere entertainment (Mateas \& Chen, 2006), one can find educational games, games for health, political games, advergames, ecogames, games for change, and many others. This innovative application of digital games in the past two decades has not only gained the attention of game developers and players but has also become the focus of interest of scholars. Since then, funding agencies have also invested a significant amount of resources in supporting the study of the application of serious games in many different domains.

In this edited volume, we narrow the scope of attention by focusing on what game theorist Ian Bogost (2007) has called 'persuasive games', that is, gaming practices that combine the dissemination of information with attempts to engage players in particular attitudes and behaviors. This implies a focus on the-mostly positive-effects of persuasive games as intended by their designers. As an aside, this also means that this volume does not address the negative effects often attributed to entertainment games, for example regarding violence and addiction (Elson \& Ferguson, 2014; Kneer, Jacobs, \& Ferguson, 2018; Raessens \& Goldstein, 2005).

Bogost's Persuasive Games: The Expressive Power of Videogames (2007) was not the first attempt to explain the persuasive potential of digital games. While authors such as Gonzalo Frasca (2007) had already theorized on how games could be used and were being used for persuasion, Bogost's book became a landmark because it was seen as the starting point of the procedural school that often used a utopian discourse about the possibility of designing digital games to change the attitude or behavior of players, including discourses supporting the idea that digital games could change 


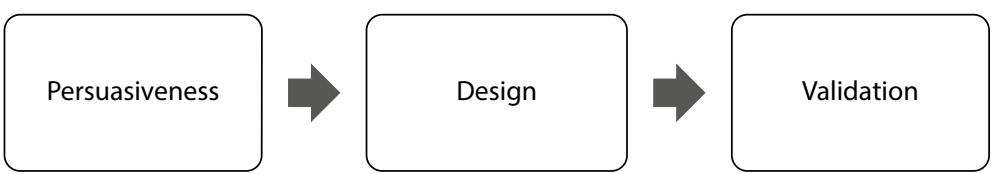

Figure 1.1: The research pillars Persuasiveness, Design, and Validation, and their subsequent relations.

the world for the better (McGonigal, 2011). Bogost's optimistic perspective on the persuasive potential of procedural rhetoric - that is, the capacity of digital games to persuade players through rule-based representations-was criticized by Sicart in his paper Against Procedurality (2011), which initiated a vivid academic debate about the persuasive potential of digital games.

This volume aims to contribute to this debate by offering a multifaceted reflection on persuasive gaming, that is, on the process of these particular games being played by players. The purpose is to better understand when and how digital games can be used for persuasion by further exploring persuasive games and some other kinds of persuasive playful interaction as well. The book critically integrates what has been accomplished in separate research traditions to offer a multidisciplinary approach to understanding persuasive gaming that is closely linked to developments in the industry by including the exploration of relevant case studies. As combining the contributions of different theoretical traditions has been rather uncommon in game studies, this volume intends to cross boundaries in research and practice.

We organized the contributions to this volume under three pillars, with each pillar amounting to an accumulation of expert knowledge (see Figure 1.1). The first pillar on persuasiveness critically assesses previous and recent theory and research on persuasive gaming and proposes a multidimensional persuasive approach as a theoretical extension that is needed in the contemporary gaming landscape. The second pillar, design, highlights research on design principles, which are understood to be the defining properties of persuasive games. The final pillar on validation incorporates both previous research on effects of persuasive games and the case-based evaluation of new games and their impact. The arrows in Figure 1.1 indicate the relationships between the three pillars, underlining that ideas about persuasiveness inspire design principles of games and that these games can be validated with respect to their impact. The feedback arrow shows that in the ideal case, the results of validation research are incorporated in theories about persuasiveness and in game design principles.

In the following sections we will discuss each pillar in detail. 


\section{Persuasiveness}

The study of persuasive communication began in ancient Greece and has a history of more than two millennia. The ancient scholars Plato and Aristotle framed rhetoric as a technique for oral persuasion, which was followed by the notable Roman scholars Quintillian and Cicero. It was Cicero who described rhetoric as a 'speech designed to persuade' (quoted in Burke, 1969, p. 49). Many centuries later, the development of mass media facilitated the broadening of the concept of rhetoric beyond oratory.

Although the focus of the study of persuasive communication has been predominantly focused on verbal strategies, the development of film, television, and visual advertisement have favored the expansion of the term. The philosopher Kenneth Burke (1987-1993) was the first to acknowledge the persuasive potential of nonverbal domains. 'Wherever there is persuasion', he wrote, 'there is rhetoric. And wherever there is "meaning", there is "persuasion"' (Burke, 1969, p. 172). Burke's work gave rise to the study of persuasiveness in many different domains, which also increased interest in visual rhetoric, understood as the art of using imagery and visuals persuasively.

Research on how audiences are persuaded by audio-visual media content is currently dominated by Petty and Cacioppo's Elaboration Likelihood Model (1986). Although a few game scholars have used ELM in their research (Malliet \& Martens, 2010), most scholars have employed a different approach by identifying the unique properties of digital games that require special attention in order to understand the way they convey meaning (e.g., Bogost, 2007; De la Hera, 2013; Ferrari, 2010; Frasca, 2007). In Bogost's classic volume (2007), procedural rhetoric was presented as the prime mechanism responsible for successful game-based persuasion.

Since the publication of Bogost's first two books (2006, 2007), procedural rhetoric has been the focus of attention of many scholars working on persuasive strategies in digital games (e.g., De la Hera, 2017; Ferrara, 2013; Ferrari, 2010; Flanagan, 2010; Heide \& Nørholm, 2009; Seiffert \& Nothhaft, 2015; Swain, 2007). What interests proceduralists is the way in which symbol manipulation of processes that initially appear unexpressive may result in a higher order of expression. However, some authors have identified shortcomings in these proceduralists' assertions (De la Hera, 2019; Heide \& Nørholm, 2009; Nelson, 2012).

In his book, Bogost (2007) claimed that digital games are a unique medium for persuasion not comparable to traditional media. This claim was criticized 
by authors such as Miguel Sicart (2011), who doubted the unique persuasive potential of procedural rhetoric taking into consideration the interactive nature of digital games and the fact that the player is required to make decisions and create a personal experience.

The section on persuasiveness in this volume starts by revisiting the debate about the value of procedural rhetoric in order to build upon the lessons learned and further develop our understanding of persuasiveness in relation to digital games. Predicting back in 2007 a near future in which games would be a primary tool for persuasion, Bogost critically reflects in Chapter 2 upon his predictions in this volume, acknowledging that not everything happened in the way he expected. Sicart also takes a different approach in his contribution (Chapter 3 ) in which he critically reflects on the playful design of mobile applications and the implications that this design approach has for our daily practices and routines.

This volume also delves into the middle-ground perspective of game scholars such Mark J. Nelson, who has stated that the 'proceduralism and play-centrism debate is too simple' (2012, para. 2) and that the two approaches are complementary. This approach is the starting point of Chapter 4 in which De la Hera and Raessens argue that additional perspectives are necessary to understand how persuasive games convey their intended meaning. Scholars defending this approach state that although procedural statements are useful in understanding how meaning can be authored in the rules of the game, it is important to acknowledge that other persuasive dimensions can complement procedural rhetoric in conveying meaning through digital games (see Figure 1.2) (De la Hera, 2019).

A similar approach is taken by Kaufman and his colleagues in Chapter 5 in which they defend the idea that game-based interventions are enhanced when the persuasive message of the game is not the focal point of the design but rather is interweaved within the game's content or the context of play. Moreover, if we aim to understand how persuasive games can realize the outcomes intended by their designers, it is clear that not only the context in which games are played but especially the role that players take in the process of persuasion should be taken into consideration. In an earlier publication, Raessens (2009) emphasizes this by using the concept of dispositif as developed within film studies to argue that the process of making meaning within digital games 'is really influenced by the ways in which configurations of technology, user positioning, desire, media text, and context take shape in specific games' (2009, p. 507). 


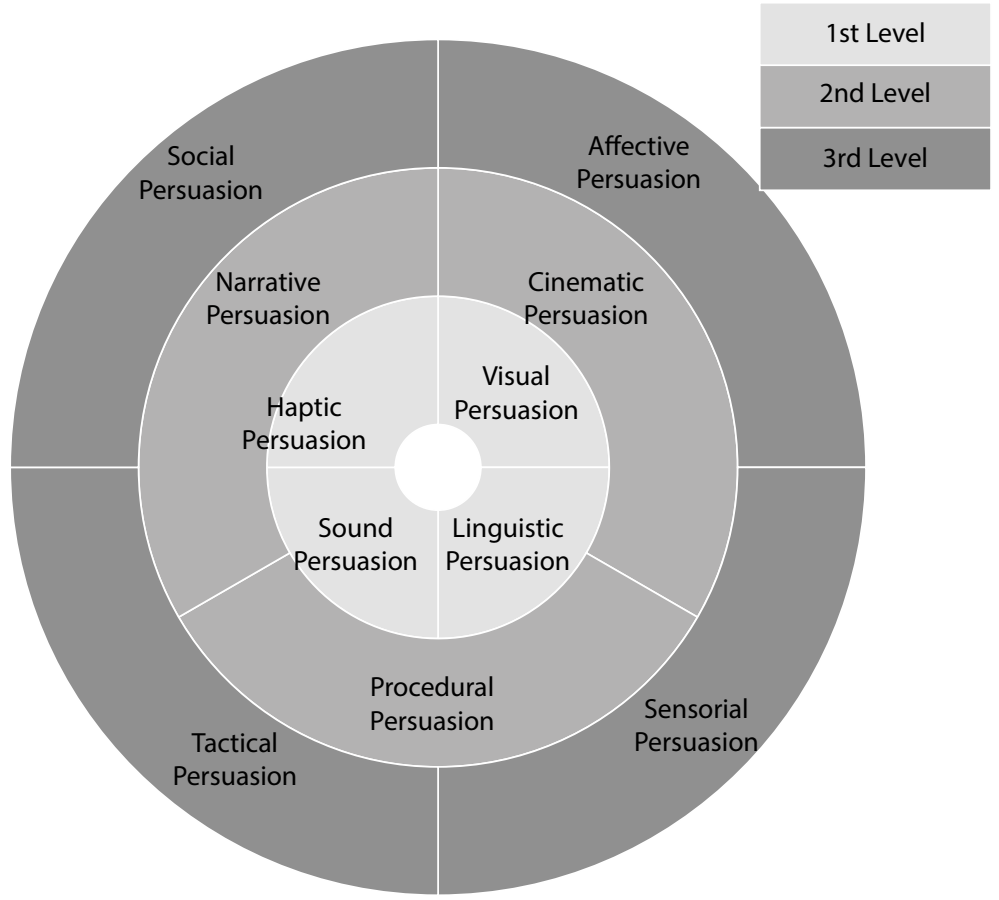

Figure 1.2: Theoretical model: persuasive communication through digital games (from De la Hera, 2019).

\section{Design for persuasive games}

In this section we will shift our focus from a descriptive to a more prescriptive perspective by discussing how we can design for persuasive gameplay. Modern game design is often guided by the influential MDA model that formalizes three distinct layers: Mechanics, Dynamics, and Aesthetics $(\mathrm{Hu}-$ nicke, Leblanc, \& Zubek, 2004). The Mechanics are the game affordances that invite specific players' actions and behaviors and are determined by the algorithms and representation of data in the game. The Dynamics concern the interactive processes unfolding between the game and the player while the Aesthetics entail the player's experiences elicited by the game, including his or her emotional responses.

Although game design generally prioritizes the game's mechanics, the MDA model helps us to understand that designing a game does not only entail considerations on the level of mechanics: 'By moving between MDA's three levels of abstraction, we can conceptualize the dynamic behavior of game systems' (Hunicke et al., 2004, p. 5) that results in player experiences (aesthetics). 


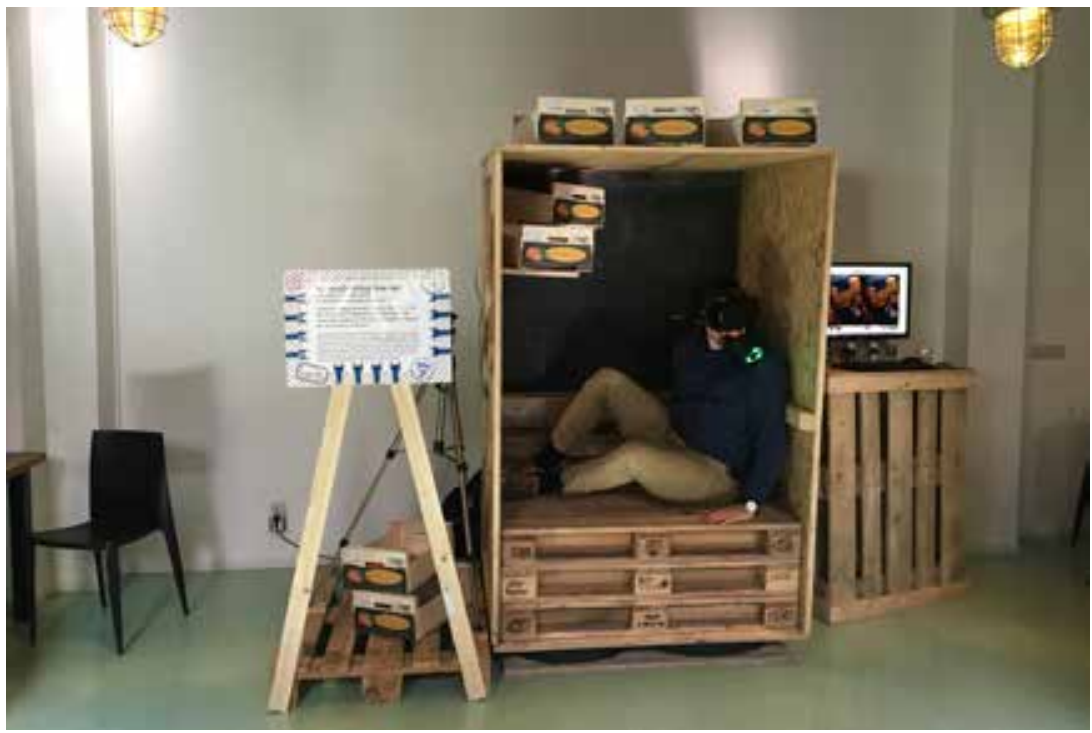

Figure 1.3: Design, using virtual reality, for empathy-arousing persuasive games (from Kors et al., 2016).

Here, we will redress the MDA balance towards a focus on the player, which is consistent with the argument developed in the previous sections of this volume. Consequently, the emphasis is on the dynamics and aesthetics, which prepares the ground for developing a design theory as well as a design practice that aim to translate player experiences into design requirements. The designer and the player each have a different perspective. The game designer takes the mechanics as the starting point, cascading to the other layers. The player's perspective focuses on the level of aesthetics, that is, designing a game that is appealing for its players. In between is the layer of dynamics where, arguably, the perspectives of designer and player meet (Ferri, Hansen, Heerden van, \& Schouten, 2018).

Our focus on the active or engaged player also has consequences for game design that aims to realize persuasive goals. As research underlines, persuasive communication is a process of learning and internalization (Fogg, 2003; Ryan \& Deci, 2000) rather than a process of simply transferring a message (e.g., a thought, an idea, an opinion) to an audience. When we translate this to the context of game design, we see that processes of persuasion are shaped predominantly at the level of dynamics. When we subsequently take the aesthetics into account, we must acknowledge that user experiences can be different from player to player and are often dependent on players' characteristics, while they may also be influenced by the context of play (Hansen et al., 2019). These multiple influences are taken into account 
in game design practices such as profiling, user studies, play session and monitoring practices, and also lately by allowing more open design strategies (e.g., game jams), where the lines between designer and player blur. Game designers Zimmerman and Chaplin (2013) have already observed that co-design and participatory design strategies were becoming increasingly popular among designers. Designing persuasive games with the aesthetics in mind also spurs the exploration and employment of novel interactions and technologies, thus changing the persuasive game design landscape over the past decade. With the advent of mobile computing, ubiquitous computing, and immersive technologies, persuasive games are no longer bound to the computer screen. For instance, designers and researchers have a keen interest in exploring persuasive games as part of the smart home (Gamberini et al., 2012) or the use of virtual reality to have players feel and understand the struggles of another (Kors, Spek, Ferri, \& Schouten, 2018).

Another example is the virtual reality (VR) game A Breathtaking Journey discussed in Chapter 6 (see Figure 1.3) in which players virtually inhabit the perspective of a refugee to gain a better understanding of their situation and conditions. Designer Martijn Kors and his colleagues describe how the details in the design enabled players to deeply engage with the role of people seeking refuge in Europe. It is a mixed-reality game that is meant to arouse empathy for refugees. The VR game A Breathtaking Journey was developed in a context of civic engagement, initiated by Amnesty International. The collaboration with Amnesty aimed to explore how interactive media could help motivate people to change or reinforce attitudes towards human rights-related issues. As a future design opportunity, the designers identify empathic relationships with one's own avatar as a still understudied possibility for persuasion.

The rapid development of game design, including its practices and principles, is reflected in the variety of methodologies used. Game designer Lindsay Grace (Chapter 7) argues that designers should go beyond the artifact of the game by taking different levels of persuasive play into account. Grace distinguishes three levels of persuasion: micro, macro, and meta-persuasion. At the first level, games and playful interactions can be seen as artifacts that aim to employ persuasive play. At the level of macro-persuasion, Grace looks at the cultural and societal impact of games and play in fostering citizen participation, for example, or supporting the formation of a community. Meta-persuasion is the least obvious of the three, originating partly as the byproduct of macro and micro-persuasion in applications of games and play in non-play contexts, such as in the construction of fake news or the application of gamification strategies in a commercial environment. 
Where Grace presents different levels of game design, Menno Deen concentrates in Chapter 8 on the process of co-design. He argues that producing a phenomenological narration of the design process enables designers to reflect on design decisions, which may result in suggestions for possible strategies for designing persuasive games at the micro level. Moreover, this methodology illuminates implementation issues that can only be identified in the actual co-design practice. Using co-design is particularly helpful in designing games that deal with contemporary problems related to gender identities, LBGHT issues, and the abuse or discrimination of minorities, for example. Deen's argument is underlined by discussing the design process of VilDu?!, a game or therapeutic tool for sexually abused children that is used in clinical practice.

In the final chapter of this section on design, Sun Joo Ahn develops yet another perspective on the context of games by presenting immersive virtual environments (IVEs). She discusses the importance of three different characteristics of virtual reality for persuasion: presence, shared experiences through perspective-taking, and compressing or accelerating time under virtual conditions. The research in this chapter can be linked to the case of $A$ Breathtaking Journey because it shows that the result with respect to the impact of embodied experiences in IVEs are promising.

\section{Validating the effects of persuasive games}

Persuasive games are designed with the purpose of realizing particular goals. In Bogost's classic volume (2007), procedural rhetoric was presented as the prime mechanism responsible for successful game-based persuasion. Since then, De la Hera (2019) has developed a theoretical argument in favor of a wider set of persuasive dimensions (see Figure 1.2) that were also used to disentangle the design of some games to determine their persuasive properties (Jacobs, Jansz, \& De la Hera, 2017). In the past decade, a handful of validation research tracks have emerged to investigate the theoretical claims made. These tracks are concerned with the games' effects on players, aiming to answer the question: Do persuasive games actually 'work'? In other words, do persuasive games succeed in realizing their intended outcome?

Most validation researchers embedded their work in the established field of persuasion research (Perloff, 2017; Petty \& Cacioppo, 1986), conceptualizing persuasion as a process of cognitive elaboration in which the game's message is reflected upon consciously to a lesser or greater degree. Research on the outcomes of persuasive games has generally focused on a change in players' 


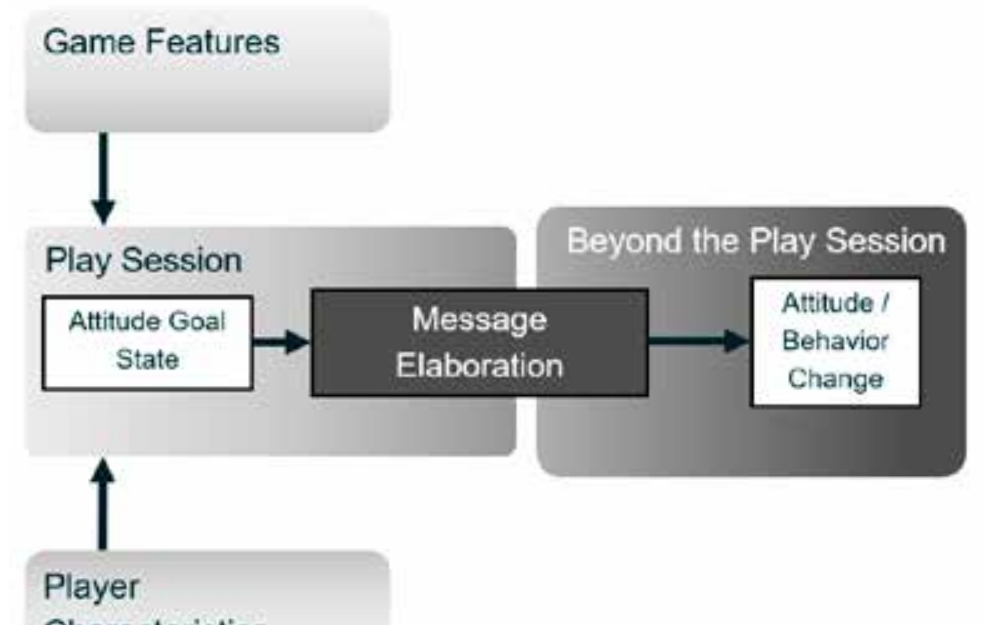

Figure 1.4: The process of persuasion from a validation perspective (adapted from Jacobs, 2017).

attitudes because attitudinal change precedes behavioral outcomes (e.g., Ajzen, 1991; O'Keefe, 2002; Petty \& Wegener, 1999). The types of attitudes involved depends on what the game is about and how the argument is presented. Following Jacobs (2017), we use the game's attitude goal state (AGS) to refer to all attitudes a certain game intends to influence. The AGS can consist of one attitude (e.g., 'refugees deserve support'), but it can also be more abstract, for example when a multi-layered topic (such as climate change) is addressed. In almost all cases, game designers embed the AGS purposively in the design of the game (Siriaraya, Visch, Vermeeren, \& Bas, 2018).

Figure 1.4 summarizes the key aspects of persuasive gaming in context from a validation perspective. Successful play causes players to elaborate on their perception of the AGS. This elaboration continues beyond the immediate context of play, resulting in attitude change, which may in turn lead to a change in behavior. Next, the figure shows that the occurrence of elaboration is also dependent on how the game is designed (its features) and what kind of person the player is. With respect to these characteristics, Rita Orji and her colleagues have proposed matching the gameplay and experience of persuasive games to the players' personalities. For example, players scoring high on an extraversion scale would enjoy games that allow them to personalize their experience more than those scoring high on a neuroticism scale (Orji, Nacke, \& Di Marco, 2017).

The emerging tradition of validation research gives reason for optimism. Many of the persuasive games that have been tested show small but 
noticeable influences on players' attitudes. In Chapter 10, Jacobs and Jansz provide an overview of what has so far been accomplished and what earlier research teaches us about the best way to study the effects of persuasive games. Effects were observed on how players think, both in the short term (Kampf \& Cuhadar, 2015; Peng, Lee, \& Heeter, 2010) and weeks after play has finished (DeSmet et al., 2018; Ruggiero, 2015). As with any kind of mediated intervention, other studies reported a lack of effects, or effects confined to specific game elements (Soekarjo \& Oostendorp, 2015; van't Riet, Meeuwes, van der Voorden, \& Jansz, 2018). Chapters 11 and 12 of this book present the results of in-depth analyses of the effects of two individual games, Urgent Evoke (Wichmand, Chapter 11) and Against All Odds (Wertley \& Soliz, Chapter 12).

It is promising that validation researchers also compared persuasive games with other media-for example, persuasive texts (Gutierrez et al., 2014; Peng et al., 2010; Ruggiero, 2015; Soekarjo \& Oostendorp, 2015), videos (Jacobs, 2016, 2017), or a combination of these (Steinemann, Mekler, \& Opwis, 2015; van 't Riet et al., 2018). These results confirm the persuasive potential of games but also show that in some cases, non-interactive media perform better. It is particularly timely to continue researching the effects of different media because such a comparative approach is close to the day-to-day reality of many people where they are flooded with persuasive attempts employing a rich variety of mediated sources. At the same time, it is necessary to continue research with no-treatment control conditions in order to establish whether particular persuasive games work at all.

The field of validation research would really advance if future studies would focus more on investigating the effects of specific game features. For example, the effects of either using narrative persuasion or procedural rhetoric were studied by comparing two games that aimed to convey the same message: Power and Control (Sain, 2011) and Another Chance (Another Kind, 2015), which were published by Jennifer Ann's Group, a non-profit charity whose aim is to combat teen dating violence (Jacobs, Kneer, \& Jansz, 2019). Another avenue for improvement is foregrounding the context of play to determine differential effects. Previous research established the differences between playing Poverty is Not a Game (iMinds, 2010) at home or in school (Bleumers et al., 2012; De Grove, Van Looy, Neys, \& Jansz, 2012). Lee and his colleagues focus in Chapter 13 on a specific context by analyzing the persuasive powers of games that are used in the training of employees in high-stake professions. But the contextual dependencies may even be more relevant when a specific context has far-reaching consequences, for example when the persuasive game is presented in an environment that partially 
simulates the living conditions of disabled persons (Gerling, Mandryk, Birk, Miller, \& Orji, 2014). One could argue that the player characteristics in Figure 1.4 are another contextual determinant because personality features co-determine the occurrence of elaboration while they simultaneously belong to a reality outside the persuasive game.

\section{Conclusion}

It is more than a decade ago that Ian Bogost published Persuasive Games (2007). Ever since, research on persuasive gaming has developed in multiple directions, with some researchers acknowledging Bogost's emphasis on procedural rhetoric, and others developing additional perspectives or criticizing the focus on game features. The steady growth of this research domain has enabled us to compile this volume based on the acquired theoretical insights with respect to persuasiveness (Part 1), combined with advanced notions about designing persuasive games (Part 2), and including the results from validation research (Part 3). In this introduction, we aimed to show that the three pillars are not independent silos but rather part of the same construction. Hence our emphasis on the feedback relations between all subsequent pillars (see Figure 1.1) is focused on the combination of contributions coming from different theoretical traditions, which results in a multidisciplinary approach to the understanding of persuasive gaming. We now turn to the substance of this volume, that is, the chapters in which a rich variety of scholars discuss their contributions to the blossoming field of persuasive gaming in context.

\section{References}

Abt, C. (1970). Serious Games. New York, NY: Viking Press.

Ajzen, I. (1991). The theory of planned behavior. Organizational Behavior and Human Decision Processes, 5o(2), 179-211. https://doi. org/10.1016/0749-5978(91)90020-T

Another Kind. (2015). Another Chance [Digital Game].

Bleumers, L., All, A., Mariën, I., Schurmans, D., Van Looy, J., Jacobs, A., Willaert, K., de Grove, F. (2012). State of Play of Digital Games for Empowerment and Inclusion: A Review of the Literature and Empirical Cases. https://doi.org/10.2791/36295

Bogost, I. (2006). Unit Operations: An Approach to Videogame Criticism. Cambridge, MA: MIT Press. 
. (2007). Persuasive Games: The Expressive Power of Videogames. Cambridge, MA: MIT Press.

Burke, K. (1969). A Rhetoric of Motives. Berkeley, CA: University of California Press. De Grove, F., Van Looy, J., Neys, J., \& Jansz, J. (2012). Playing in School or at Home? An Exploration of the Effects of Context on Educational Game Experience. Electronic Journal of E-Learning, 10(2), 199-208. Retrieved 10 September 2020 from https://eric.ed.gov/?id=EJ985422.

De la Hera, T. (2013). A Conceptual Model for the Study of Persuasive Games. Proceedings ofDiGRA 2013:DeFragging Game Studies, 1-15. https://doi.org/10.2307/4448051

- (2017). Persuasive Gaming: Identifying the Different Types of Persuasion Through Games. International Journal of Serious Games, 4(1), 31-39. https://doi. org/10.17083/ijsg.v4i1.140

- (2019). Digital Gaming and the Advertising Landscape. Amsterdam: Amsterdam University Press.

DeSmet, A., Bastiaensens, S., Van Cleemput, K., Poels, K., Vandebosch, H., Deboutte, G., Herrewijn, L., Malliet, S., Pabian, S., De Bourdeaudhuij, I. (2018). The Efficacy of the Friendly Attac Serious Digital Game to Promote Prosocial Bystander Behavior in Cyberbullying Among Young Adolescents: A Cluster-Randomized Controlled Trial. Computers in Human Behavior, 78, 336-347. https://doi. org/10.1016/j.chb.2017.10.011

Elson, M., \& Ferguson, C.J. (2014). Twenty-Five Years of Research on Violence in Digital Games and Aggression: Empirical Evidence, Perspectives, and a Debate Gone Astray. European Psychologist, 19(1),33-46. https://doi.org/10.1027/1016-9040/a000147 Entertainment Software Association. (2018). Essential Facts: About the Computer and Video Game Industry. Retrieved 29 September 2020 from https://www.theesa. com/wp-content/uploads/2019/o3/ESA_EssentialFacts_2018.pdf

Ferrara, J. (2013). Games for Persuasion: Argumentation, Procedurality, and the Lie of Gamification. Games and Culture, 8(4), 289-304. https://doi. org/10.1177/1555412013496891

Ferrari, S. (2010). The Judgment of Procedural Rhetoric [PhD Thesis]. School of Literature, Communication, and Culture. Georgia Institute of Technology, Georgia. Retrieved 20 September 2020 from https://smartech.gatech.edu/ handle/1853/33915

Ferri, G., Hansen, N.B., Heerden van, A., \& Schouten, B.A.M. (2018). Design Concepts for Empowerment through Urban Play. In Proceedings of the DiGRA 2018 Conference. Turin, Italy. Retrieved 20 September 2020 from http://www.digra. org/digital-library/publications/design-concepts-for-empowerment-throughurban-play/

Flanagan, M. (2010). Creating Critical Play. In R. Catlow, M. Garrett, \& C. Morgana (eds.), Artists Re:thinking Games, pp. 49-53. Liverpool: Liverpool University Press. 
Fogg, B.J. (2003). Persuasive Technology: Using Computers to Change What We Think and Do. (Vol. 5). https://doi.org/10.4017/gt.2006.05.01.009.0o

Frasca, G. (2007). Play the message. Play, Game and Videogame Rhetoric [PhD Thesis]. IT University Copenhagen. Retrieved 29 September 2020 from https://www.igdb. com/articles/play-the-message-play-game-and-videogame-rhetoric-dissertation Gamberini, L., Spagnolli, A., Corradi, N., Jacucci, G., Tusa, G., Mikkola, T., Zamboni, L., Hoggan, E. (2012). Tailoring Feedback to Users' Actions in a Persuasive Game for Household Electricity Conservation. In: Bang M., Ragnemalm E.L. (eds.), Persuasive Technology. Design for Health and Safety. PERSUASIVE 2012. Lecture Notes in Computer Science, vol. 7284. Berlin/Heidelberg: Springer. https://doi. org/10.1007/978-3-642-31037-9_9.

Gerling, K.M., Mandryk, R. L., Birk, M., Miller, M., \& Orji, R. (2014). The Effects of Embodied Persuasive Games on Player Attitudes Toward People Using Wheelchairs. In Proceedings OfThe 32nd Annual ACM Conference on Human Factors in Computing Systems - CHI '14, pp. 3413-3422. https://doi.org/10.1145/2556288.2556962

Gutierrez, B., Kaatz, A., Chu, S., Ramirez, D., Samson-Samuel, C., \& Carnes, M. (2014). 'fair Play': A Videogame Designed to Address Implicit Race Bias Through Active Perspective Taking. Games for Health Journal, 3(6), 371-378. https://doi. org/10.1089/g4h.2013.0071

Hansen, N. B., Dindler, C., Halskov, K., Iversen, O.S., Basballe, D.A., \& Schouten, B. (2019). How participatory design works: mechanisms and effects. In Proceedings of the 3ist Australian Conference on Human-Computer-Interaction, pp. 30-41. https://doi.org/10.1145/3369457.336946o

Heide, J., \& Nørholm, S. (2009). Playful Persuasion. The Rhetorical Potential of Advergames. Nordicom Review, 3o(2), 53-68. Retrieved 20 September 2020 from https://www.nordicom.gu.se/en/tidskrifter/nordicom-review-22009/ playful-persuasion-rhetorical-potential-advergames

Hunicke, R., Leblanc, M., \& Zubek, R. (2004). MDA:A Formal Approach to Game Design and Game Research. AAAI Workshop - TechnicalReport (Vol. WS-04-04). Retrieved 20 September 2020 from https://users.cs.northwestern.edu/ hunicke/MDA.pdf Iminds. (2010). Poverty is not a Game [Digital Game].

Jacobs, R.S. (2016). Play to Win Over: Effects of Persuasive Games. Psychology of Popular Media Culture, 7(3), 231-240. https://doi.org/10.1037/ppmooo0124

_ (2017). Playing to Win Over: Validating Persuasive Games [PhD Thesis]. ERMeCC-Erasmus Research Center for Media Communication and Culture. Retrieved 20 September 2020 from https://repub.eur.nl/pub/102769/

_ , Jansz, J., \& De la Hera, T. (2017). The Key Features of Persuasive Games: A Model and Case Analysis. In Kowert, R., and T. Quandt (eds.), New Perspectives on the Social Aspects of Digital Gaming: Multiplayer 2. London: Routledge. https:// doi.org/10.4324/9781315629308 
Kneer, J., \& Jansz, J. (2019). Playing Against Abuse: Effects of Procedural and Narrative Persuasive Games.Journal of Games, Self, and Society, 1(1), 97-120. https://doi.org/10.1184/R1/7857578

Juul, J. (2010). A Casual Revolution: Reinventing Video Games and Their Players. Cambridge, MA: MIT Press.

Kampf, R., \& Cuhadar, E. (2015). Do Computer Games Enhance Learning About Conflicts? A Cross-National Inquiry into Proximate and Distant Scenarios in Global Conflicts. Computers in Human Behavior, 52(Suplement C), 541-549. https://doi.org/10.1016/j.chb.2014.08.008

Kneer, J., Jacobs, R.S., \& Ferguson, C.J. (2018). You Could Have Just Asked: The Perception of Motivations to Play Violent Video Games. Studies in Media and Communication, 6(2), 1. https://doi.org/10.11114/smc.v6i2.3389

Kors, M.J.L., Ferri, G., van der Spek, E.D., Ketel, C., \& Schouten, B.A.M. (2016). A Breathtaking Journey: On the Design of an Empathy-Arousing Mixed-Reality Game. In Proceedings of the 2016 Annual Symposium on Computer-Human Interaction in Play - CHI PLAY' '6. https://doi.org/10.1145/2967934.2968110

— Partaker or Victim. Delineating Three Perspectives to Empathic Engagement in Persuasive Games Using Immersive Technologies. In Proceedings of the 2018 Annual Symposium on Computer-Human Interaction in Play Companion, pp. 493501. https://doi.org/10.1145/3270316

Malliet, S., \& Martens, H. (2010). Persuasive Play: Extending the Elaboration Likelihood Model to a Game-Based Learning Context. In R. van Eck (ed.), Interdisciplinary Models and Tools for Serious Games, pp. 210-226. Hersey, PA: IGI Global.

Mateas, M., \& Chen, S. (2006). Serious Games: Games that Educate, Train and Inform. Boston, MA: Thompson.

McGonigal, J. (2011). Reality is broken: Why Games Make Us Better and How They Can Change the World. New York, NY: Penguin.

Nelson, M.J. (2012). Sicart's “Against Procedurality”. Retrieved 23 August 2018, from http://www.kmjn.org/notes/sicart_against_proceduralism.html

O'Keefe, D.J. (2002). Persuasion: Theory and Research. New York, NY: SAGE Publications.

Orji, R., Nacke, L.E., \& Di Marco, C. (2017). Towards Personality-Driven Persuasive Health Games and Gamified Systems. In Conference on Human Factors in Computing Systems - Proceedings, pp. 1017-1027. New York, NY: ACM Press. https://doi. org/10.1145/3025453.3025577

Peng, W., Lee, M., \& Heeter, C. (2010). The Effects of a Serious Game on Role-Taking and Willingness to Help.Journal of Communication, 6o(4), 723-742. https://doi. $\operatorname{org} / 10.1111 / \mathrm{j} .1460-2466.2010 .01511 . \mathrm{X}$ 
Perloff, R.M. (2017). The Dynamics Of Persuasion: Communication and Attitudes in The 21st Century (5th ed.). New York, NY: Routledge. https://doi. org/10.4324/9781315657714

Petty, R.E., \& Cacioppo, J.T. (1986). The Elaboration Likelihood Model of Persuasion. Advances in Experimental Social Psychology, 19, 123-205. https://doi.org/10.1016/ Soo65-26o1(08)60214-2

, \& Wegener, D.T. (1999). The Elaboration Likelihood Model: Current Status and Controversies. In S. Chaiken \& Y. Trope (eds.), Dual Process Theories in Social Psychology, pp. 37-72. New York, NY: Guilford Press. https://doi.org/10.1016/ Soo22-4405(97)00003-4

Raessens, J. (2009). The Gaming Dispositif: An Analysis of Serious Games from a Humanities Perspective. In Serious Games: Mechanisms and Effects. https://doi. org/10.4324/9780203891650

- (2014). The Ludification of Culture. In M. Fuchs, N. Scharpe, R. Paolo, \& S. Fizek (eds.), Rethinking Gamification, pp. 91-114. Lünenburg: Hybrid Publishing Lab.

— , \& Goldstein, J.H. (2005). Handbook of Computer Game Studies. Cambridge, MA: MIT Press.

Ritterfeld, U., Cody, M., \& Vorderer, P. (2009). Serious Games: Mechanisms and Effects. New York, NY: Routledge. https://doi.org/10.4324/9780203891650

Ruggiero, D. (2015). The effect of a persuasive social impact game on affective learning and attitude. Computers in Human Behavior, 45, 213-221. https://doi. org/10.1016/j.chb.2014.11.062

Ryan, R.M., \& Deci, E.L. (2000). Self-Determination Theory and the Facilitation of Intrinsic Motivation, Social Development, and Well-Being. American Psychologist, 55(1), 67-78. https://doi.org/10.1037/0oo3-066X.55.1.68

Sain. (2011). Power and Control [Digital Game].

Seiffert, J., \& Nothhaft, H. (2015). The Missing Media: The Procedural Rhetoric of Computer Games. Public Relations Review, 41, 254-263. https://doi.org/10.1016/j. pubrev.2014.11.011

Sicart, M. (2011). Against Procedurality. Game Studies, 11(3). Retrieved 10 October 2019 from http://gamestudies.org/1103/articles/sicart_ap

Siriaraya, P., Visch, V., Vermeeren, A., \& Bas, M. (2018). A Cookbook Method for Persuasive Game Design. International Journal of Serious Games, 5(1). https:// doi.org/10.17083/ijsg.v5i1.159

Soekarjo, M., \& Oostendorp, H. van. (2015). Measuring Effectiveness of Persuasive Games Using an Informative Control Condition. International Journal of Serious Games, 2(2), 37-56. https://doi.org/10.17083/ijsg.v2i2.74

Steinemann, S.T., Mekler, E.D., \& Opwis, K. (2015). Increasing Donating Behavior Through a Game for Change. In Proceedings of the 2015 Annual Symposium on 
Computer-Human Interaction in Play. New York, NY: ACM Press. https://doi. org/10.1145/2793107.2793125

Swain, C. (2007). Designing Games to Effect Social Change .DiGRA 2007 Conference. Digital Games Research Association. Retrieved 14 October 2019 from digra.org/ wp-content/uploads/digital-library/07311.09363.pdf

Van 't Riet, J., Meeuwes, A.C., Van der Voorden, L., \& Jansz, J. (2018). Investigating the Effects of a Persuasive Digital Game on Immersion, Identification, and Willingness to Help. Basic and Applied Social Psychology, 4o(4), 180-194. https:// doi.org/10.1080/01973533.2018.1459301

Zimmerman, E., \& Chaplin, H. (2013). Manifesto: The 21st Century Will Be Defined By Games. Kotaku. Retrieved 24 May 2018, from https://kotaku.com/ manifesto-the-21st-century-will-be-defined-by-games-1275355204

\section{About the authors}

Teresa de la Hera is Assistant Professor of Persuasive Gaming at Erasmus University Rotterdam, where she is a member of the Research Cluster Gaming Matters. Her expertise is focused on understanding how digital games are used as media for communication and tools for engagement, motivation, and persuasion. She is the author of Digital Gaming and the Advertising Landscape (2019), published by Amsterdam University Press.

Jeroen Jansz is Full Professor of Communication and Media at Erasmus University Rotterdam. He holds the Chair of Communication and Media in the Department of Media \& Communication. His research aims to understand and explain the changing relations between media consumers and media producers with a specific focus on digital gaming.

Ruud Jacobs researched the validation of persuasive games at the Erasmus University Rotterdam as part of the research project Persuasive Gaming. From Theory-Based Design to Validation and Back (NWO). He now works as an Assistant Professor of communication and technology at the University of Twente, investigating why people play serious games.

Ben Schouten is an artist/designer as well as a full professor in playful interaction at the department of industrial design of the Eindhoven University of Technology. His research focuses on play and participatory design for social innovations and citizen empowerment through bottom-up approaches. He 
has co-edited five volumes and conference proceedings and has co-authored more than 100 publications.

Joost Raessens is Chair and Full Professor of Media Theory at Utrecht University and scientific director of the Utrecht Center for Game Research. He is co-editor of the volumes The Playful Citizen (2019) and Playful Identities (2015), both published by Amsterdam University Press, and the Handbook of Computer Game Studies (The MIT Press, 2005).

Martijn Kors is a doctoral candidate at the Industrial Design Department of the Eindhoven University of Technology in Eindhoven, the Netherlands. His design-oriented research focuses on studying the intersections between persuasive game production, system immersion, and the employment of empathy-arousing appeals to shape how players think and feel about issues in reality. 


\section{Part I}

The Study of Persuasion

Through Digital Games 



\title{
Introduction to Part I
}

\author{
Joost Raessens \& Teresa de la Hera
}

This part of the book sheds light on how we can understand persuasion through digital games from a theoretical perspective. The authors in this section discuss different perspectives on how digital games can be used with the intention of influencing the attitude and behavior of players. Their contributions go beyond the utopian and dystopian discourses on persuasive games and the debate on their validity that centered game scholars' attention when this new field of research was emerging more than ten years before this volume was published. Therefore, we reflect critically on the role and the value of digital games to be used as a medium for persuasion, we pay attention to the medium itself and its characteristics, and we provide theoretically grounded propositions on how to work with persuasion within digital games. Together, these chapters not only demonstrate that persuasive games have become part of our contemporary media culture, they also provide a critical discussion of what the role of persuasive games is in our current media landscape. These chapters also pay special attention to the role players have in the process of persuasion through digital games and how their characteristics and performances should be taken into consideration in this process.

Ian Bogost opens the first part of this book by revisiting his claims on procedural rhetoric published in his book Persuasive Games in 2007. In his chapter entitled 'Persuasive Games, A Decade Later', he admits to having been wrong in some of his predictions about the role persuasive games would play in the actual social and political context. Bogost acknowledges that persuasive games have not become the tools of complex knowledge he predicted. He also admits that persuasive games are not being widely used, as he expected, to make arguments about what worldly behaviors are desirable or undesirable and on how to address the big, important problems in the world. The author nonetheless stresses the value of continuing to study and discuss persuasive games as a concept.

In Chapter 3, Miguel Sicart broadens the discussion of the persuasive potential of digital games by paying attention to playful persuasion and critically reflecting on the playful design of services apps. In 'Playing an Automated World', he uncovers the social, political, and ethical consequences of embedding a playful design in these apps, and with this purpose in mind, he claims that by applying methods and concepts from toy and game design, 
this engaging and persuasive software might be eroding forms of labor and social connection.

Teresa de la Hera and Joost Raessens pay attention in Chapter 4 to how players' characteristics should be taken into consideration when designing digital games with persuasive intentions. In 'Looking Beyond Persuasion Through Rule-Based Representations in Digital Games', the authors propose that persuasive goals and game goals should be aligned differently depending on whether the main purpose of the game is to shape, to reinforce, or to change players' attitudes. In addition, De la Hera and Raessens critically discuss the academic debate on procedural rhetoric and reflect on how designing digital games that can be both persuasive and engaging for players.

In the last chapter of this section, Geoff Kaufman, Mary Flanagan, and Max Seidman defend the idea that persuasive games greatly benefit from using a nuanced, less explicit approach to convey their persuasive messages. In 'Creating Stealth Game Interventions for Attitude and Behavior Change', the authors present a model of 'embedded design' that offers concrete strategies to convey persuasive messages in games tackling social issues. The strategies proposed as part of this model are focused on bypassing players' psychological barriers to try to foster a more positive mindset towards the persuasive message. 


\title{
2. Persuasive Games, A Decade Later
}

\author{
Ian Bogost
}

\begin{abstract}
More than a decade ago, Bogost invented the concept of 'procedural rhetoric' - the idea that games and software can make arguments through their mechanics. Even earlier, he founded a studio called Persuasive Games that adopted procedural rhetoric as a design philosophy. These ideas have had some influence on game studies and design, including finding their way into the title of this volume. And yet the promise of persuasive games in the world as a force that would introduce systems literacy to the mass media has not been successful. What happened, and what-if anything — can be done about it?
\end{abstract}

Keywords: procedural rhetoric; persuasive games; design philosophy; procedurality; games as literacy

It has been a decade since I published Persuasive Games, my book on how computer games and simulations make arguments and express ideas (Bogost, 2007). The game studio that I founded (which I also named Persuasive Games) to produce those sorts of games for politics, education, business, and more is now fifteen years old. These milestones are sufficiently notable, for me at least, that I've been giving them considerable thought.

It's not just my idea, of course. In the book, I drew on lots of precedents in theory and design. Clark Abt's work concerned non-computational serious games in the early 1970s that long predated the digital versions that became popular around the turn of the millennium (Abt, 1970). Meanwhile, designers like Chris Crawford were making political games two decades before the publication of Persuasive Games. And simulation games-from Sim City (Electronic Arts, 1993) to the delightful, esoteric titles about farm machinery and cheesemaking that line the coffers of Steam - all try to capture the world and represent it, in part, in game form.

Hera, T. Dela, J. Jansz, J. Raessens, B. Schouten, Persuasive Gaming in Context. Amsterdam: Amsterdam University Press, 2021 DOI 10.5117/9789463728805_CHO2 
My version of the idea was always pretty simple: games and simulations are systems of interlocking parts and behaviors. The world is also made of interlocking parts and behaviors. This parallel structure gives games a unique purchase on representing how things work in the world. And because games are representational, they can also depict how things should work - that is, they can make arguments about which worldly behaviors are desirable or undesirable. This approach to argument seemed different enough from other forms of rhetoric — verbal, visual, and so forth — that I suggested a new category for it: procedural rhetoric, namely rhetoric arising from processes, behavior, and models.

This argument has had some considerable influence. People read the book and, to some extent, still have to read it if they want to pursue the theoretical canon of game studies. As the design philosophy for the studio, it produced some interesting work, including the first official game for a U.S. presidential candidate (which I worked on with my colleague Gonzalo Frasca and his studio in Uruguay), along with games about airport security, consumer debt, disaffected workers, the petroleum industry, suburban errands, a pandemic flu, and tort reform. Millions of people played some of those games, and others were collected or exhibited internationally. I appeared as a guest on the TV program 'The Colbert Report' and received lots of attention.

Yet looking back, I have to admit that this influence has not been as substantial and important as I'd hoped it would be in the late 199os and early 20oos. Indeed, to some extent, there is a reckoning to be had for persuasive games.

The promise - my promise - was that the big, important problems in the world were also complex systems. Issues like the climate, health, economics, and social justice offer examples, but even small-scale phenomena also work like complex systems. Running a local shop or understanding the impact of a new municipal ordinance also muster systemic behavior. At the studio, for example, we made a game about portioning for a franchised ice-cream parlor. The system in this case was quite odd and unique: The texture and viscosity of various ice cream flavors were important, as were the topology of its vat (as workers served from it over the course of a shift) and that phenomenon's interaction with the economics of the franchise, including both customer and worker satisfaction. The idea, at least in my head, was that almost everything involved systemic behaviors that couldn't be explained by simple, declarative statements.

This truth seemed so clear to me in the early 2000 s that I made some remarkable predictions. It's worth emphasizing how long ago that was-before 
Facebook, before iPhone, before YouTube. Blogs were the big thing, along with Google, which hadn't yet gone public. Among my ill-advised notions was the claim before the 2004 U.S. presidential election that every candidate for major office would have their own PlayStation-quality game by 2008 , outlining their policy positions in simulated form - a playable platform. Instead, by 2008, we got YouTube and video-based campaigning, and then Facebook and data-based campaign targeting. By 2016, that data took on a weaponized form in the United Kingdom, the U.S., Myanmar, and elsewhere. The extraction, sale, and manipulation of data seemed to become the standard method for social 'engagement'. As I write this in 2018, it's pretty clear that I could not have been more wrong in thinking that debates fueled by procedural rhetoric would emerge as a new standard in political discourse.

It's not just politics, either. Games were supposed to produce 'system literacy' and help people embrace complexity. The game designer Eric Zimmerman, writing with the journalist Heather Chaplin, imagined the 21st century as the 'ludic century', in which playful sophistication would make prior forms of communication outmoded (Zimmerman \& Chaplin, 2013). Instead, we ended up with ever-simpler discourse, built around shorter and more impatient sound bites. Television condensed into online video. Discourse metastasized in comment sections, then Twitter arguments. This situation seems only to have gotten worse. Moreover, it's unclear how it might be stopped. After the Cambridge Analytica crisis of 2018, which was just one of countless extractions of Facebook data in the interests of social manipulation, Facebook executives, including CEO Mark Zuckerberg, were asked to testify before the U.S. Congress and the U.K. Parliament. Despite this, just a few weeks later at the annual Facebook developers' conference, Zuckerberg was joking about the whole affair. The company's stock price, meanwhile, had recovered from much of the losses suffered from its Cambridge Analytica fiasco, thanks to enormous quarterly profits. Furthermore, in general, very few people have quit Facebook. How could you? It's where two billion people socialize.

Around 2010, I started to realize an inconvenient truth: that in order for people to be persuadable by games, it wasn't enough to have a few interesting games that represented the potential for a revolution in knowledge and understanding. Part of this realization came from my own work in news games, both theoretical and applied. After failing to scale up a promising partnership in game development with the New York Times, largely for organizational-political reasons, I began surveying that field (with my students and thanks to support from the John S. and James L. Knight Foundation). One of the lessons we discussed in the book resulting from 
that work was the need to scale up (Bogost, Ferrari \& Schweizer, 2010). You can't invent televised news broadcasts by trying one or two out and seeing how it goes. An entire social practice must be cultured around the form, involving habits of time and attention. The same is true for games for news, politics, education, or anything else.

And yet, you can't will that sort of scale. The rest of the media environment would have to support it. And that support would require change. In fact, it might even need the decline of the media formats of the 2oth century for systems-driven procedural rhetoric to have any hope of taking its place. Even more positive developments in media failed to take the form of playable systems with procedural rhetorics. Instead, smartphones filled up with the media forms of the 2 oth century: words, images, moving images, and audio. That is, more or less, what everyone makes and consumes for computers. It's the same kind of media that they've been producing since the turn of the century, even if it's tuned and updated for modern means of fashioning and disseminating it. The revolution of systems thinking never came, and by the look of things, it isn't poised to do so anytime soon. Instead, 2oth-century media grew ever more powerful, recombining with computers and smartphones into new versions of themselves.

It's interesting to pause and think about the approach many of us used to discuss the non-entertainment uses of games in those days. We were conducting historical research, to a point, and contemporary criticism, too. But efforts like Persuasive Games were also future forecasts. They imagined a media experience that wasn't yet present; one that might instead be lurking just over the horizon. This is a tricky gambit for a futurist, let alone a critic or scholar. It's one thing to wax philosophical about forthcoming trends and then to collect massive consulting or speaking fees for the privilege of espousing them; it's a little different to do so in the context of research and commentary.

Consider James Paul Gee, for example, whose 2003 book What Video Games Have to Teach Us About Learning and Literacy made a major splash in this field (Gee, 2003). He had already had two full careers in two different disciplines, linguistics and literacy, by the time he turned his focus to games in the early 200os. Gee is a very strategic thinker, as I'd learn when I got to know him in those early days of persuasive games, some fifteen years ago. The position he presented was one about the potential of games as literacy tools, or as models for learning. He never presented learning games as a fait accompli, a goal realized. Rather, he used careful readings of commercial games like Ninja Gaiden (Tecmo, 1988) as evidence for the premise that good 
games provide better teaching than other kinds of learning experiences. Yet what they teach, at least for now, is just how to play the game.

I think Gee's approach to this matter was far more tactical than mine. That is, he knew that realization was a much more complex and squirrely problem than visioning. He'd had a lot more experience at that time than I had, after all. The joke was on me and others who tried so hard to realize our ideas rather than rising above them and viewing the patterns of social behavior that might make them work or not. It's possible that folks like me, supposedly righteous for doing both theory and practice, may have actually closed our eyes to the truth of our successes and failures because we were so head-down trying to realize them.

The promise of persuasive games was tempting, in part because it seemed so structurally plausible. Given complex systems in the world, what better way to depict them - and create tools to revise them - than by translating and representing them with computational systems? This offered a tidy, symmetrical view of the world that might, in retrospect, have betrayed its reliance on logic and reason.

Yet ironically, and in reality, it was emotion and novelty that drove much of the interest in this work. In my own case, a tremendous amount of the value and benefit of persuasive games came not from the ends they supposedly facilitated through procedural rhetoric but from the idea of that promise. Hey, a game about educational funding! A game about contagious diseases! The headlines followed suit: 'It's Not Just Fun and Games', or the like. In this environment, games functioned on a rhetorical register alright, but not on a procedural one; instead, they worked on the level of their impression and concept. Elizabeth Losh calls this rhetorical use of digital tools virtualpolitik; that is, the digital media themselves are not deployed to their functional ends but are held up as evidence of a type of labor and creativity (Losh, 2009). The fact of persuasive games' existence became their primary effect. Talking about the idea of a game on, say, poverty or politics replaced — or at least far outstripped - any exploration of the system in the game itself. In many cases, particularly when my own games have received attention in the press, it's clear that far fewer people ever thought to play them than thought to think about doing so.

When they are played, the effects of persuasive games are often very different than the ones their creators might expect. One of my favorite examples of the genre is Molleindustria's (2006) McDonald's Videogame, a scathing critique of the multinational fast-food industry. The game demonstrates the abject corruption required to maintain the profitability and manageability of a large global food company. It's a terrific example of procedural rhetoric, and both stylish and even fun to boot. 
In the game, players control fields in South America where cattle are raised and soy is grown, a factory farm where cows are fed, injected with hormones and controlled for disease, a restaurant where workers have to be hired and managed, and a corporate office where advertising campaigns and board members set corporate policy.

As play progresses, costs quickly outstrip revenue, and the player must take advantage of more seedy business practices. These include razing rainforests to expand crops, mixing waste as filler in the cow feed, censuring or firing unruly employees, and corrupting government officials to minimize the public outcry against such actions.

But many players-especially those who are technically minded and who enjoy mastering their video games-find themselves lamenting the difficult job of McDonald's executives rather than becoming incensed by their corrupt corporate policies. I've had a number of students make this observation about the game, in fact. 'Wow, I really empathized with the CEO of a big company. They have it rough.'

When Molleindustria released a similar game some years later, it sailed a slightly different tack. The game, Oiligarchy (Molleindustria, 2008), was about the global petroleum industry and its collusion with government at all levels. Paolo Pedercini (the individual creator who publishes his work as Molleindustria) posted a 'postmortem' with text and images that explain the premise of the game: peak oil, supply and demand, imperialism, and so forth. It included the following statement:

This document, written after the release of Oiligarchy, attempts to outline the major game design choices we faced and provide footnotes and additional documentation to the parts that reference real-world situations or events. Since the inception of the Molleindustria project we argue that game design is never an ideologically neutral process: games, as every other cultural product, reflect the designers' beliefs and value systems. And this is particularly visible in games that claim to 'simulate' actual non-deterministic situations (Pedercini, n.d).

In Pedercini's defense, he produced that document in part to fulfill the requirements of his MFA degree, which required this sort of documentation. But even so, the materials exist, and they risk overtaking the work. And at least the game itself was good and worthwhile as a game. Many of my own games have been far less adept procedural arguments than Pedercini's, and yet they have been effective enough successes from the vantage point of virtualpolitik. 
Take one of my own games, a documentary version called Fatworld (Persuasive Games, 2007), about the politics of nutrition. The game was a strange mix of Animal Crossing (Nintendo 2001), the terrific Nintendo series about living in an animal village, and the work of the nutritional historian Marion Nestle. You can create a character that has physical properties like avatars in every game. But then they also have health properties, including girth and medical histories, as well as food allergies and other predispositions. That character then gets dropped into a simulated world in which socioeconomics have an impact on the choices players can make with the character they designed.

So, players can design recipes and meals for their characters, but they have to shop for, and therefore be able to afford, the component parts of those meals. That might be easier or harder depending on one's financial situation. Players can also mess with the world's non-player characters by deciding what they can and can't eat-you could create a Fried Chicken Emporium or a Wheatgrass Hut, or anything in between, although the market may impact the viability of those choices.

Players can also alter public policy, experimenting with regulation politics and subsidies. These subsidies recombine with socioeconomic contexts to create nutrition effects, for example the relationship between fast food and low-income diets. So, you could attempt to ban trans fats (or even vegetables, if you prefer), although political influence is strongly tied to financial access.

What's more, knowledge about your character's health and wellness only becomes available if you can afford healthcare, although policies for subsidized or even socialized medical care are also possible if the will of the community supports it. In this way, the feedback mechanism necessary to do well in the game is subject to the game's own simulated politics, such that adjusting the choices and opportunities for your character may be harder or easier depending on how good and how frequently that health feedback loop can be run.

Then, eventually, your character dies and you can start over again.

This is precisely the kind of game that I've been endorsing for years now-one that embraces the complexity of a sociopolitical issue and in so doing characterizes it honestly. Yet this game did terribly. Part of it was our fault - the budget and timeframe didn't match the ambition-but part of it was related to reception and expectations, just as was the case for Molleindustria's titles.

It's interesting to compare Fatworld to one of its contemporaries, another game-related effort to address nutrition and obesity that followed shortly thereafter: the Apps for Healthy Kids contest. This was one of the initiatives in Michelle Obama's efforts to address diet and wellness from the White House. 
One of the winners, called ZisBoomBah, was 'an innovative website that challenges conventional wisdom and develops tools to empower children and inspire parents to live a fun, active and healthy life'. ZisBoomBah's free online tool 'Pick Chow!' allows children to create meals by dragging and dropping foods. This is clown-town stuff; just another obvious 'choose the carrot, not the candy bar' simulator that couldn't possibly teach anyone anything about the mechanics, let alone the politics, of nutrition and obesity.

And yet it was massively successful. Why? Because addressing the complexity and political intractability of nutrition was not really the White House's goal. Instead, it wanted to signal technological adeptness and literacy. In the Obamas we had an executive branch that knew what an app was and could make a colorful website with hip, big form-fields. Once again, the project works on a different rhetorical register than the procedural.

In both these cases, the games' ability to do the work of procedural representation was short-circuited by media distractions, by orthogonal media situations that resist games' fundamentals while furthering their own power and effect.

A question arises, for Pedercini, for me, and for all designers of persuasive games: If the game is incapable or inadequate when it comes to doing this work on its own, and if the traditional, un-systemic, presumably outmoded media of text and image are necessary or even better, then why are we making games at all? The whole practice risks becoming an aesthetic exercise. And that's only the case when the games are any good!

Many years ago, during the height of the early success of persuasive games (the idea, the book, and the studio), I saw the writer Steven Johnson speak at an industry conference. He had just published his book Everything Bad is Good for You, which took up games and other somewhat reviled popular media that are unexpectedly adept at making people smarter (Johnson, 2005). During his talk, Johnson noted that he'd seen my name and my stuff-persuasive games — and admitted that he just didn't think that games could ever become a really effective persuasive medium, at least not one as effective as language — text and orality, anyway, the bread-and-butter of this writer's universe.

At the time I stewed on this observation, dismissing it as unimaginative naysaying. Yet, since then, I've wondered if Johnson had a point. Today, I spend much more of my time writing words about the world than I do making games that depict its operation. Have I given up? No, not at all. Although, in some ways, the next generation of persuasive games, in theory and in practice, probably needs to come from different voices than those who inaugurated the form. Instead of fatalism, I suggest a kind of stark 
realism for that next chapter. Like it or not, persuasive games remain an aspirational media form. A form with potential, as James Paul Gee would say, of games and learning; a potential yet to be realized.

A while back, it became a tech-industry cliché to talk about 'changing the world'. Everything from an app that orders pet food to a new way to purify water became equally implicated in the process of world-changing. The idea became so comical that it was even sent up on the HBO series Silicon Valley; at a simulated rendition of the TechCrunch Disrupt conference, socially inept entrepreneurs mumbled about their inscrutable products and services, always announcing in the process how it was going to 'change the world'.

That aspiration—really a marketing hook, not a goal—has bled into all aspects of contemporary life now that computers, smartphones, and apps are all pervasive. Games were lured in, too. 'Can games change the world?' read one headline back in 2012, far closer to the heyday of persuasive games' first generation. This is ironic, because that very notion was always anathema to the gambit of persuasive games. The very idea of changing the world as a simple principle is incompatible with the premise that games have a unique power to reject simplicity, demonstrate complexity, and help people to mistrust singular answers. The quandary now poses a paradox, one that the next generation of persuasive-games theorists and designers will have to reconcile and resolve.

Some might read these words and see pessimism or defeat, which are sentiments I really don't mean to embrace. So, let me end with two thoughts. The first is a reminder, one I issue to myself as much as to anyone who might read this. The promise of procedural rhetoric and persuasive games, to me at least, was one of a moderated rationalism. It wasn't meant to descend into brusque positivism, where logic would win out over emotion. That's just rational extremism - something best left to economists. Instead, it was meant to embrace realism. In particular, it was meant not to shy away from the messy, true nature of tough, even intractable social and political systems. To dismiss that approach also means rejecting the project. Instead, we must face it head-on. How can games become the tools of complex knowledge that many of us have promised, while also participating in, and altering, the media circumstances that resist and even destroy complex knowledge? That is a challenge for the future-for me, for you, for all of us who make, play, and advocate for persuasive games.

And that leads me to the second thought, which is a reflection rather than a directive: My self-critique risks forgetting where the bar was set for games about unfamiliar topics, from statements of policy to health appeals 
and from playable education to characterizations of human experience. I didn't invent this idea, of course - versions of those games had been around for decades. Infocom text-adventures had danced with themes of human loss and regret, edutainment titles had become cultural icons, and politicalstrategy games had been discussed in the New York Times. Yet at the start of the new millennium, those games had mostly been forgotten, except as specimens from an alternate timeline.

Even so, at that moment, new tools, audiences, and opportunities opened up. One of the most important contributions of persuasive games as a concept might have been amplifying that moment in time and giving it some lift. The idea that you could make games for learning or business or policy was part of that notion one subsumed into so-called serious games and games for change as well. But those tools were-and remain-largely instrumental. While I don't believe there's anything wrong with instruments, persuasive games also promised something more: that games could embrace the soul of political, social, and personal speech; that they could do so unapologetically and with style and persona, too; and that they could be like the social-realist novel or the lyrical poem or the documentary film. I wasn't responsible for the blossoming of those flowers in the decade thereafter, but I do think that the most important contribution of persuasive games as an idea, a studio, a book, and a theory might have involved watering those buds at a moment when they particularly craved it. And you know, maybe that's enough. Just as procedural rhetoric argues that the important thing is to see how a constellation of influences, causes, and effects conspire to produce complex outcomes, so persuasive games themselves had a role in such a bigger play. One that's not over yet, either.

\section{References}

Abt, C. (1970). Serious Games. New York, NY: Viking.

Bogost, I. (2007). Persuasive Games: The Expressive Power of Videogames. Cambridge, MA: MIT Press.

— , Ferrari, S., \& Schweizer, B. (2010). Newsgames. Journalism at Play. Cambridge, MA: MIT Press.

Electronic Arts (1993). Sim City [Computer Game].

Gee, J.P. (2003). What Video Games Have to Teach Us About Learning and Literacy.

New York, NY: Palgrave.

Johnson, S. (2005). Everything Bad is Good for You: Today's Popular Culture is Actually

Making Us Smarter. New York, NY: Riverhead. 
Losh, E. (2009). Virtualpolitik: An Electronic History ofGovernment Media-Making in a Time of War, Scandal, Disaster, Miscommunication, and Mistakes. Cambridge, MA: MIT Press.

Molleindustria (2006). McDonald's Videogame [Online Game].

Molleindustria (2008). Oiligarchy [Online Game].

Nintendo (2001). Animal Crossing [Console Game].

Pedercini, P. (n.d). Oiligarchy Postmortem. Retrieved 10 October 2018 from http:// www.molleindustria.org/oiligarchy-postmortem/.

Persuasive Games (2007). Fatworld [Online Game].

Tecmo (1988). Nija Gaidem [Console Game].

Zimmerman. E. \& Chaplin, H. (2013). Manifesto: The 21st Century Will Be Defined By Games. Kotaku. Retrieved 10 October 2018 from https://kotaku.com/ manifesto-the-21st-century-will-be-defined-by-games-1275355204.

\section{About the author}

Ian Bogost is the author of Persuasive Games (MIT Press, 2007) and an award-winning game designer. He is the Ivan Allen College Distinguished Chair in Media Studies and Professor of Interactive Computing at the Georgia Institute of Technology, where he also holds an appointment in the Scheller College of Business. Bogost is also Founding Partner at Persuasive Games LLC, an independent game studio, and a Contributing Editor to The Atlantic. 



\title{
3. Playing an Automated World
}

\author{
Miguel Sicart
}

\begin{abstract}
In these days of technological revolution, we have two parallel trendsautomation and gamification/playful design — driving the ways we design technologies for consumption. In fact, play design has been heralded as the approach that will make the computer-mediated forms of work into pleasurable experiences. However, this very assumption is troublesome if we question what we mean by play. In this piece, I will question the assumption that making something feel like play needs to be questioned, that it has some ethical implications that need to be reflected upon, and that even though the assumption might be correct, it is so only if we properly define what we want from playing everything.
\end{abstract}

Keywords: playful design; ethical implications; playful apps; playful persuasion

\section{Introduction}

I can't remember the last time I visited a bank to transfer money. I also can't remember the last time I used an ATM to check my account. I can't recall when I last used my laptop for any banking activity. I can't remember when I last spoke to a taxi dispatcher, and I haven't communicated once with my children's school in person when they're sick.

All these mundane activities have been mediated through apps. This is a 'comfortable' new world in which my interactions with the human beings that mediate services are limited to those cases of catastrophic failures of software systems. The systems I interact with are convenient and are designed to be pleasant, engaging, and playful.

When I describe these systems as playful, I'm referring to a particular quality of their design, a set affordance that is aesthetic-driven that

Hera, T. Dela, J. Jansz, J. Raessens, B. Schouten, Persuasive Gaming in Context. Amsterdam: Amsterdam University Press, 2021 DOI 10.5117/9789463728805_CHO3 
allows users to use these services not just as functional devices but also as pleasurable experiences. In Play Matters (2014), I defined playfulness as an attitude toward the world that brings the benefits of play (expressiveness, appropriation) to activities that are, in fact, not play. Playful services want to feel like playthings, even if they are ultimately serving another purpose.

One recent example of this approach is IKEA Place, an app developed by the Swedish furniture giant that uses augmented reality (AR) to allow users to place furniture from their catalogue in a 'real space'. Users can select the furniture they are interested in and see how it would fit in a space thanks to an AR representation in the camera feed of their mobile phones. IKEA Place is far from being a game; it's a sales instrument designed to streamline the experience of the IKEA catalogue and to make a visit to the shop more efficient or even redundant. Yet the placement of objects in space feels very playful.

In this chapter, I want to critically examine playful design-services apps. I want to question why these are designed to be experienced from a playful, engaging perspective and what social, political, and ethical consequences this has for playable mundane software in different speculative playable futures. I want to explore the idea that this engaging, mundane, comfortable software might be eroding forms of labor and social connection and is doing so by applying methods and concepts from games and toy designs. The chapter addresses the following questions: What happens when services become apps? What happens when these service apps are designed as playful experiences? And what sociopolitical and ethical effects might a playable automated future have?

The chapter addresses issues of gamification and the playful design of service apps from the perspective of the philosophy of technology. To do this, post-phenomenology and the philosophy of information provide the conceptual background that allows me to frame the analysis of these applications from a critical perspective. I will also engage critically with design research, play theory, and game studies. Finally, I will try my hand at a more speculative form of research, imagining possible playable futures that illustrate the role that play might have in shaping how technologies mediate the world. My goal is to conduct an analysis of future dystopias or utopias so that we can ask questions of our technologies, practices, and ideologies before we reach a point at which we have no opportunity to do so. I don't claim to be right or to hold an empirically proven truth. I simply maintain that we need to think things through and to ask questions before we fully embrace the pains and pleasures of playable automated services. 
The chapter is divided into five sections, including this introduction. Section two presents the concept of 'soft automation', which I'll use to connect software-based (self-)services with the broader phenomenon of automation. Section three introduces the problems that arise when using play as an interface to 'soft automated' services. The section will focus on how play's capacity to create order as well as redefining action through formal rules might be a problematic design approach. Section four presents the dystopian summary of a future dominated by playable self-services; using a variation of speculative design thinking, I will present what a future of playable services might look like.

This chapter is an exploration, a probe launched toward the incoming present and the potential futures of play and playable technologies. The point of this chapter is to ask questions, to reflect, to think aloud why we need to think carefully about using play to engage people with automated self-services. This chapter wants to draw a picture of the kind of research that we need to address these issues; the kind of work that's ahead of us.

\section{Soft automation}

It's fashionable these days to be worried about automation (Vallor, 2014). Ranging from concerns about disappearing jobs (Srnicek \& Williams, 2015) to the fear of an AI planet (Bostrom, 2016), we're witnessing a revolution in the way labor is organized and eliminated. And we should be afraid of the quotidian, mundane forms of automation that we're silently allowing to take hold of our daily life.

There is a subtle, insidious, and unexpected form of automation that's changing the way we interact with services. The explosion of AI techniques for natural language processing and image recognition and the ubiquitous presence of smartphones have changed how we interact with services. Through concepts like 'big data', states and corporations alike are finding solutions to make their services more accessible to users by developing software that can be installed on a smartphone, is available $24 / 7 / 365$, and allows users to benefit from engaging with these services when they please.

This has led to a transformation of the service sector toward a self-service sector, in which service providers are increasingly software companies with some customer-facing operations, yet are focused on providing digital access to their services through software applications. As I mentioned in the introduction, banks have led the way in transforming their services into apps. In countries like Denmark, most citizen services can be accessed 
online or via apps, and this trend is extending to all interactions with all services, from the police to the nursery.

From a user perspective, there's much to like in this revolution: there are no queues; we don't need to schedule our lives around opening hours; and the simplicity of an app just makes ordinary transactions fee/ less complicated. Everything is just more comfortable. Yet, like with all comforts, there might be a trade-off that we aren't considering, one that has important implications.

The transition to self-services involves a displacement of labor from specialist workers with privileged access to the inner workings of services to users being deputized to perform service operations with the aid of software. Professions that used to be the stalwarts of the middle class and didn't require a long, academic education, like bank teller or clerk, are disappearing as we users accept doing that work ourselves, without any intermediaries. The promise of these vanishing intermediaries hides the threat of eliminating those service specialists—-such as travel agents-who understood how complex systems operated and could navigate them for us.

I call the transformation of services into self-services via software applications 'soft automation'. Soft because it's based on software, and soft because it doesn't bring forth the fears of the terminator that will kill us or the car-assembling robot that will steal our jobs. What's more, because these software-based services depend on algorithms to translate complex systems into a user-friendly interface, soft automation has the effect of displacing labor from specialist workers to users.

Why should we care about soft automation in a book dedicated to persuasive play and games? Research into games as engagement tools has shown that they can be used to increase satisfaction, engagement, and learning in users (Fuchs, Fizek, Ruffino \& Schrape, 2014; Walz \& Deterding, 2015). There are indications that some forms of gamification and game-based learning increase engagement and improve education results. If we know that games and playable interactions are engaging, what better framework do we have with which to create applications that interface complex systems to users in appealing ways?

Habitica (HbitRPG, 2013) is a classic example of a take on gamification. On their GitHub page, the developers of this project define it as an 'open source habit building program which treats your life like a Role Playing Game' (HabitRPG, 2018). Habitica is essentially a to-do-list application that uses mimicry and make-believe to engage users in keeping track of their habits and potentially changing them. Habitica uses the conventions of computer role playing games to generate this effect: players create a character that levels up and acquires new items as tasks are fulfilled, from going to the gym to taking out the trash. 
Using a game structure derived from role-playing games as a motivational instrument is a very clever design tool: role-playing games are based around the fulfillment of tasks, and personal development can be framed as a way of leveling up. The application of the design and aesthetic language of games makes Habitica an example of classic gamification, namely the application of game structures to activities with the goal of making them pleasurable.

Gamification could be applied to making soft automation processes more engaging by making them playful. It wouldn't be surprising to see game designers and service providers collaborate in the development of pointbased systems that would help users to transition from using services to self-service experiences. But if (or when) this happens, we'll need to question the consequences of framing self-services as playable experiences that use the design vocabularies of games and playthings. These are questions that will have an impact on the way we experience technology as well as in the role of gamification design, serious games, and play design in the future.

\section{Playing services}

Play is a powerful instrument to engage users of digital systems, make them more attached and engaged to their activities, and translate repetitive, boring, and mildly complex tasks into something more pleasurable (Bogost, 2007). The promise of play is that by making things feel like games, and by structuring the activity using game-design techniques, users can play the world and therefore feel more engaged with the activity at hand (Deterding, 2012).

This approach, while controversial (Lieberoth, 2015), has been shown to be somewhat effective (Márquez Segura et al., 2016), but there's a trade-off that I believe we haven't properly discussed yet. Let's go back to classic play theory and read the work of Huizinga ([1938] 1992) and his successors (Henricks, 2016) from the perspective of a culture that wants to have play everywhere.

Huizinga characterized play by describing it as a separate, autotelic activity that imposes order in the world. It's not my intention to discuss here the concept of the magic circle again (Consalvo, 2009). My focus is Huizinga's argument that play is a voluntary activity that takes place in bounded space and time, that has a purpose of its own, and that is separate from the real world. Whatever happens in play is a consequence of the activity itself, and what motivates us to engage with this activity is derived from the pleasures that we can experience when playing. 
When we play a game, for example, its rules help us impose an order to our activity. We play in turns, we know who can win and who can lose, and we have a limited yet meaningful set of actions we can take in order to interact with the game and other players. Of course, things aren't as clear-cut as this description suggests. Goffmanian research on games and play (Goffman, 1961) shows how play is also a social event that requires from players their active participation in maintaining social relations that are both intrinsic to the play activity and related to the social relations of players outside the game (Deterding, 2017).

Huizinga provided a set of concepts to describe and define play, and contemporary play scholars like me are still dealing with them. We've extended Huizinga's core notions of play by addressing the permeability of the magic circle, the relationship between play and games (Malaby, 2007), or the phenomenon of 'bleed' in live action role-playing games (Waern, 2011). And yet, we're still discussing play and games through the concepts presented in Huizinga's Homo Ludens. I don't mean to downplay the importance of these concepts, nor do I want to propose an alternative; instead, I want to highlight that the way we analyze and design play is deeply rooted in issues of separateness, the autotelic purpose of play, and its imposition of order in the world.

Furthermore, despite efforts to describe the morality of games and play (Sicart, 2009), we still need to properly address how an autotelic activity can have an effect in our moral lives. Huizinga famously declared that play is beyond morality, and Caillois and other researchers questioned the ontological status of play once the 'real world' starts having an effect on its structures (Caillois, 2001). Similarly, Goffman pointed out how part of the social act of playing is negotiating what elements external to the activity of playing the game have an influence on playing the game and why these externalities might have catastrophic consequences for the activity of play (Goffman, 1961).

If we want to address the challenges that gamification and persuasive games face in their attempt to make play a more engaging way of interacting with services, it's essential to discuss the ontological processes that happen at play and how they shape the experiences of players/users. Huizinga's description of play, so influential in our culture, provides players with the argument that their actions when playing are solely motivated by their intention to play to experience the pleasures of play itself. The autotelic, separate nature of play, even if qualified through concepts like bleed (Waern, 2011), still justifies the 'it's just a game' argument, i.e., the co-optation of responsibility for the actions taken during play, because play is a separate activity with its own logic and purpose. 
It's precisely this argument that I find to be the most dangerous, unexplored domain when it comes to a playable future. Any plaything, from games to toys to playgrounds, is a technology designed to mediate human experience and to help structure and engage with the order that play requires to become an activity. In other words, playthings shape human experience toward the activity of play. When we apply elements of game or play design outside the domain of games, we're trying to replicate the way playthings mediate human experience. Therefore, we need to understand that process of shaping experience. To do this, I will turn now to post-phenomenology.

Post-phenomenology, a contemporary phenomenological school (Ihde, 2012), has developed a methodological approach that allows for identifying and questioning the role that a particular technology has in the experience of the world (Verbeek, 2008; Aagard, 2017). The experience of the world is always mediated by technology, shaping intentionality and helping construct subjectivities (Rosenberger, 2014). This is a world-construction process: Our experience is that of creating and being in a world in which our experience and our subjectivity become. Games are devices explicitly designed to construct that world by constraining agency and creating obstacles that focus our experience.

Much like games, computers have the capacity to organize our experience based on rules. This limits our action but also enhances it, giving it new potential meaning: running away from zombies, becoming healthier one run at a time. Computational technologies perform an operation in our experience that modifies our experience of the world: computers create worlds, much like play.

As an example, the data tracked by sensors on phones is used by fitness trackers to visualize and present that data using digital maps and graphical representations of movement, speed, and distances. Computer-mediated exercise is an activity that takes place in a computed world. Bodies and motions become those bodies and motions that can be tracked and located in the digital world of the fitness tracker.

Post-phenomenology allows us to see how computing technologies and play arrange our experience in similar ways. The technologies of play and computation not only mediate but also give sense, purpose, and meaning to the experience of the world. But this doesn't explain the risks of the 'it's just a game' argument. To fully explain my concerns, I want to examine what play and its technologies do to the world.

The relationship between computers and play can be described as a process of re-ontologization (Floridi, 2013). Information and communication technologies like smartphones and computers are re-ontologizing 
technologies that transform the nature of the world as experienced precisely because they are information machines: 'the ontology of the information technologies available [...] is the same as (and hence fully compatible with) the ontology of their objects, the raw data being manipulated' (ibid, p. 7). Re-ontologization is the 'source of some of the most profound transformations and challenging problems that we will experience in the close future, as far as technology is concerned' (ibid, pp. 6-7).

What makes computing machines catalyzers of this re-ontologization process is 'the fundamental convergence between digital resources and digital tools. The ontology of the information technologies available [...] is now the same (and hence fully compatible with) the ontology of their objects [...] in the re-ontologized info sphere, there is no longer any substantial difference between the processor and the processed, so the digital deals effortlessly and seamlessly with the digital' (ibid). Computers turn the informational world into a digital environment, and they also afford particular interactions, effectively re-shaping the nature of the world.

Play is also a way of re-ontologizing the world. Playing a game is creating and inhabiting a world, a process of rearranging things and agency so that new relationships, behaviors, and experiences can take place. Videogames give us worlds, from World of Warcraft's Azeroth to Fallout's The Wasteland. Games also give us the communal village of fear and monsters of Werewolf, or the world-about-to-die of Pandemic. Sports give us the contained worlds of stadiums and courts, with their own logic and ethics. Play is a way of creating worlds within this world.

Play is a re-ontologizing activity because it has three characteristics that allow it to re-shape the essence of the world. First, play is appropriative: to play is to take over a situation, a context, a space, and a time and make it the scene or the instrument of play. This act of appropriation is always contextual and open to negotiation: we don't take over the whole of an object or the whole of a situation but only those aspects that we find interesting, relevant, or appropriate for our goals within the activity of playing.

Second, play is autotelic. The purpose of the activity of appropriating the world is always negotiated, expressed, and applied to the situation or object play that takes over. When that purpose is fulfilled, the activity ends. To play is to play for a purpose, which is sometimes fun but sometimes is not. Play has a purpose of its own, a purpose that is explicit, argued for, and is not rigidly determined.

Finally, play is expressive. To play is not to consume or perform actions in a particular order for particular goals; to play is to produce or to perform actions in a particular order with a personal touch, for a personal reason, 
a reason that becomes expression. To play is to make a world in which that being is possible, meaningful, and creative (Sicart, 2014).

Play redefines the nature of the world and radically changes it. It reontologizes the world in more temporary, negotiated, and impermanent ways than computers but still in ways that are creatively, ethically, and politically relevant. To play is to appropriate the world to create $a$ world in which we can play and in which the activity we are engaged with is meaningful and we can express ourselves. These are impermanent worlds that have an impact but not permanence beyond the activity of play.

The computational capacity of re-ontologization is at the heart of soft automation: Software helps us to redefine tasks that require expertise and turn them into self-services in which computational agents are specifically designed to help users achieve their goals. These services are re-ontologized, their nature changed so computational systems can have degrees of agency that aid users in performing tasks.

These transformations need to be shaped in such a way that users find them palatable. Playful design, through gamification practices, helps users leverage these new computational-driven experiences. By turning the rules and algorithms that computers use to re-ontologize the world into game-like rules and playable instructions, playful design makes the experience of a computationally re-ontologized world more accessible and more interesting.

A good example of this process is Siri, Apple's voice-controlled assistant. I have in the past written about Siri as a plaything (Sicart, 2014). However, it's a critical example to return to because it illustrates how play and computation are related and how playfulness can be used to interface with a computational world. Siri is the result of an assemblage of technologies and practices, from voice recognition to cloud computing, that allows us to interact with our mobile phone just using our voice. Siri is the affable voice of a computable world.

And that's why it's so important that Siri has been designed with humor and playfulness as part of its repertoire of answers. Siri is not just a functional interface; it's a playful interface that has opinions. It can reply with limited but charming wit and seems to be willing to listening to us when nobody else wants to. A playful Siri implies an engagement with the computationally re-ontologized world that is in itself playful. It's not just an interface; it's Siri, with its quirks and opinions. It's a joyful interface, re-ontologizing through play the functions of the interface so that engagement with that computational environment is more palatable.

When we make self-services playful, when we create the activity of play, we are reconstructing a world that orients our experience based on the 
autotelic goals and actions afforded by the technologies themselves. In the next section, I will explore some possible playable futures that might be the outcome of the playful re-ontologization of computational self-services.

\section{Playable futures}

I now want to speculate about the playable, soft-automated futures that await us, in order to trace a potential future of playable technologies from the perspectives of play and automation (Frase, 2016). These potential futures could allow us to understand the potential impact of a playable future and reflect upon what a positive playable future would be like. This is a speculative design-thought experiment, with the goal being to consider models of a future in which playful soft automation has succeeded.

First, imagine that soft automation has succeeded in eliminating all intermediary jobs. In order to engage with any service, from ordering food to applying for a new passport or naming a child, people just need the right app on their phone or browser. People seldom need to interact with another person-even healthcare has been automated. The bureaucrats are all behind the curtains of highly automated systems, dealing exclusively with exceptions and malfunctions. They are the elite, shaping the way automation works and the way we experience it. They are the human gatekeepers of the algorithms. But they are as remote and arcane as the algorithms themselves.

Once all services are automated, we need to make them not just Normanfunctional, not just devices designed with Norman's usability principles in mind (Norman, 2002), but also engaging. Usability and practical concerns are not enough for people to change their habits. We need emotional design so that we find pleasure in performing these tasks through apps. This leads to a great play awareness moment: If only we can make things feel like play, people will have better lives and be more engaged in their activities. Work applications, tasks, chores; they can all be experienced as we experience toys, namely as instruments that extend our agency on the world, that give us not just increased engagement but also a feeling of appropriation of the world, of being able to self-determine the purpose of our actions.

We can imagine a future of playable services that are good to experience, that are fun and pleasurable and act like games or toys. The goals of these pleasurable services are to serve and entertain. We already have examples of this kind of service, such as Carrot's animistic designs for mobile apps (http://www.meetcarrot.com). Carrot apps are based on the idea of giving a voice to the processes and activities that the user engages with when using 
those apps. Whether it's checking their to-do lists or taking exercise, there's a character that ruthlessly comments on the users' efforts.

Carrot's application of animistic design is fundamentally playful: by creating a character with a personality that comments on player actions, Carrot designers establish an experiential relation of alterity between the user and the technology (Verbeek, 2006), using mimicry as a playful way of establishing that relationship (Caillois, 2001). In a future in which services have been automated into playful self-services, Carrot's strategy might be a dominant one, namely, to make these services feel and behave like toys so that all these mundane activities become more than tasks we have to complete.

In this future, playable services might engage us more, but they will also bring a certain type of focus on the experience. Playful economic transactions may hide how self-services eliminate a type of job that has often been considered part of the broad middle class. These services can be toys, but they might be the toys of affluence and ignorance, the self-serving self-services that isolate users in playgrounds that are well-defined, secure, and ultimately controlled. The playground can be a panopticon in which we voluntarily submit to ignorance just to experience the pleasures of play. A playable self-service world will hide the processes of re-ontologization through the pleasures of play, reducing our engagement with the meaning and consequences of our interactions, making us play ourselves to death (Postman [1985] 2005).

There is another potential playable future in which automated selfservices are functional, pragmatic applications that don't get in the way of our goals. In this possible future, we aren't expected to be emotionally engaged with these services; they are there, they are functional, and they facilitate our living in computational societies. What is the role of play in this automated society? If the software isn't playful but it works and there's no other option than to use it, why should we even think about play? In a future of efficiency, play can be an instrument to reclaim agency in automated worlds, a way of experiencing the world on our own terms.

Play can be a strategy to experience self-services as playthings. All these soft-automated systems require our complicity to work. Play gives us instruments to challenge them, to reclaim the importance of personal communication, of the pleasures in the mundane, of being recognized not by an algorithm but by a person who knows you. We love the convenience of self-services, yet we yearn for worlds that are more human, more local, and more personal: organic, gluten-free, locally sourced. Play might give us a way of satisfying some of that yearning. 
Take, for example, the application Converse, which is designed to translate languages using sophisticated speech recognition and cloud-computing technologies. Converse presents itself as more than just an instrument; it has a vibrant interface full of animations and audiovisual feedback that makes the act of translation feel like a way of connecting people with each other. Converse isn't a functional instrument but more a pleasurable form of engagement with automated translation services.

Similarly, the application Insight Heart uses augmented reality (AR) to allow users to see and navigate the cardiovascular system using their mobile devices as windows to a medically correct representation of the human anatomy. This is an instrument and a toy that leverages the magic of AR with the functionality of a learning application.

There are also aesthetic or political examples of this future. The mobile application Buy Partisan allows users to scan the barcode of groceries, figure out the political leanings of their producers and manufacturers, and therefore orient consumerism toward supporting political ideas. More aesthetically inclined is Die With Me, a chat application that will only allow users to communicate with others when their devices have $5 \%$ of battery or less. Instead of providing infinite access to communication, this app limits the available time to communicate, while at the same time extending the meaning of chatting on a phone, perhaps with strangers, for a painfully short time before the battery dies and with it the connection to the other person.

These are examples of currently available services. But perhaps the playable future we deserve, the one that would allow us to resist the pressures of soft automation and use playfulness as an instrument for resistance, should instead be found in the results list of the Stupid Shit No One Needs \& Terrible Ideas Hackathon (stupidhackathon.com). This is an event that produces terrible contraptions that make fun of the technologies that define our time. From analog takes on AR like Intestine AR (http://www.jhclaura. com/peep-rack/) to the brilliant Hypochondriapp (http://hypochondriapp. io), which will always provide the worst possible diagnosis for whichever symptoms are typed in, the Stupid Shit Hackathon provides a playful insight into what happens when we play with the computers around us.

Play allows us to define our goals in the experience of the world. In this playable future, we play the self-services: we poison the data needed to record our behavior by being silly; we cheat trackers or poke fun at the unavoidable creepiness of permanent behavioral tracking. Alternatively, we can even try different, new strategies based on the disorderly aspect of play. For example, we could photocopy and fax the screen of our portable 
device showing our bank statements, instead of using the convenient, yet impersonal, email systems embedded in finance applications.

This is a romantic take on play, perhaps a dated vision that evokes the more optimistic dreams of the New Games movement (DeKoven, 2013). I may be naive and think that we can use not just the capacity that play has to engage us but also the capacity that it has to define its own goals and let us have ownership of them and our expectations. In a future of self-services, where we are carefully and functionally guided to press the right buttons, play might be the way of making that world less mechanically mundane.

We are moving toward an automated future, and this might be a ludic century. The combination of both machines and play, I believe, will shape our future, and so thinking about the excesses we might incur could help us find livable middle grounds.

\section{Conclusions}

In this chapter, I wanted to raise some questions about playable futures. Serious games and gamification have provided us with the conceptual tools to apply game-design principles outside the domain of games. Self-services are an obvious target for playful redesigns, as they might leverage their functional mundanity with increased playable engagement. But play, given its autotelic nature, might actually stop us from understanding the societal and cultural impact that soft automation might have.

This chapter charts the poles between the optimism regarding playable technologies and a reflection on how play can be problematic. Play gives us new worlds, and computers give us new comforts. It's easy to be seduced by them, to be persuaded to use these playable services because they are not just services but because they engage not only with our needs but also with our wants. Yet we can't ignore the fact that these take place in the context of accelerating the capitalism that promotes the destruction of labor so that the accumulation of capital can be streamlined. By turning services into self-services, labor is displaced from employed specialists to users. By making users 'play' these services, we isolate them from the effects they might have in culture, society, and the commons. And so we are risking feeding a self-reinforcing, capitalist-optimizing machine.

Therefore, as a small and not very satisfactory conclusion, I want to insist on a basic idea: we need to think about the implications of making things other than games have a game feel. Games are instruments created to craft worlds in which player agency is extended and limited by clear 
rules, justified by pleasurable goals, and encapsulated in the voluntary logic of participating in the game. If we make services feel like play, we may be making them more pleasurable, but we may also be making them more separate, their rules and procedures to be interpreted as arbitrary limitations imposed on the world instead of social contracts that bind our actions to collective goods or personal goals. If we don't take these implications seriously, if we don't think through how a playful world may affect our engagement with society and its technologies, we're risking turning the world into a playground that hides in playable pleasures the harsh realities of capitalistic automation.

\section{References}

Aagaard, I. (2017). Introducing Postphenomenological Research: A Brief and Selective Sketch of Phenomenological Research Methods. International Journal of Qualitative Studies in Education, 6(30), 519-533. https://doi.org/10.1080/09518398.2016.1263884. Bogost, I. (2007). Persuasive Games. Cambridge, MA: MIT Press.

Bostrom, N. (2016). Superintelligence: Paths, Dangers, Strategies. Oxford: Oxford University Press.

Caillois, R. (2001). Man, Play and Games. Urbana and Chicago, IL: University of Illinois Press.

Consalvo M. (2009). There Is No Magic Circle. Games and Culture, 4(4), 408-17. https://doi.org/10.1177/1555412009343575.

DeKoven, B. (2013). The Well-Played Game. Cambridge, MA: MIT Press.

Deterding, S. (2012). Gamification: Designing for Motivation. Interactions, 19(4), 14-17. https://doi.org/10.1145/2212877.2212883.

—. (2017). Alibis for Adult Play: a Goffmanian Account of Escaping Embarrassment in Adult Play. Games and Culture, 3(3), 260-279. https://doi. org/10.1177/1555412017721086.

Floridi, L. (2014). The Fourth Revolution. Oxford: Oxford University Press.

- (2015). The Ethics of Information. Oxford: Oxford University Press.

Frase, P. (2016). Four Futures: Life After Capitalism. London: Verso.

Fuchs, M., Fizek, M., Ruffino, P. \& Schrape, N. (2016). Rethinking Gamification. Lüneburg: Meson Press.

Goffman, E. (1961). Encounters. Two Studies in the Sociology of Interaction. Indianapolis, IN: Bobbs-Merrill.

HabitRPG (2013). Habitica [Mobile App].

- — (2018). Habitica. Retrieved 24 September 2018 from https:/github.com/ HabitRPG/habitica. Accessed 1/3/2018. 
Henricks, T.S. (2016). Play and the Human Condition. Urbana and Chicago, IL: University of Illinois Press.

Huizinga, J. (1971). Homo Ludens. London: Beacon Press.

Ihde, D. (2012). Experimental Phenomenology. SecondEdition. New York, NY: SUNY Press. Lieberoth, A. (2015). Shallow Gamification. Games and Culture, 1o(3), 229-248. https://doi.org/10.1177/1555412014559978.

Malaby, T.M. (2007). Beyond Play: A New Approach to Games. Games \& Culture, 2(2) (2007), 95-113. https://doi.org/10.1177/1555412007299434.

Segura, E.M., Waern, A., Segura L.M., \& Recio, D.L. (2016). Playification. In CHI PLAY '76: Proceedings of the 2016 Annual Symposium on Computer-Human Interaction in Play. New York, NY: ACM Pres, 376-88. https://doi.org/10.1145/2967934.2968099.

Postman, N. (2005), Amusing Ourselves to Death:Public Discourse in the Age of Show Business. London: Penguin Books.

Rosenberger, R. (2014). Multistability and the Agency of Mundane Artifacts: From Speed Bumps to Subway Benches. Human Studies, 37(3), 369-92. https://doi. org/10.1007/s10746-014-9317-1.

Sicart, M. (2009). The Ethics of Computer Games. Cambridge, MA: MIT Press. . (2014). Play Matters. Cambridge, MA: MIT Press.

Srnicek, N., \& Williams, A. (2015). Inventing the Future. London: Verso.

Vallor, S. (2014). Moral Deskilling and Upskilling in a New Machine Age: Reflections on the Ambiguous Future of Character. Philosophy \& Technology, 28(1), 107-24. https://doi.org/10.1007/s13347-014-0156-9.

Verbeek, P.P. (2006). What Things Do. University Park, PA: Penn State University Press.

- (2008). Cyborg Intentionality: Rethinking the Phenomenology of Humantechnology Relations. Phenomenology and the Cognitive Sciences, 7(3), 387-95. https://doi.org/10.1007/s11097-008-9099-x.

Waern, A. (2011). “I'm in Love with Someone That Doesn't Exist!” Bleed in the Context of a Computer Game. Journal of Gaming \& Virtual Worlds, 3(3), 239-57. https://doi.org/10.1386/jgvw.3.3.239_1.

Walz, S.P. \& Deterding S. (2015) (eds.). The Gameful World. Cambridge, MA: MIT Press.

\section{About the author}

Miguel Sicart is Associate Professor at the Center for Computer Game Research at IT University Copenhagen. He is a play scholar with a background in philosophy of technology, literature, and game studies. Sicart is the author of Play Matters (2014), Beyond Choices: The Design of Ethical Gameplay (2013), and The Ethics of Computer Games (2009), all published by the MIT Press. 



\title{
4. Looking Beyond Persuasion Through Rule-Based Representations in Digital Games: Designing Games to Shape, Reinforce, or Change Attitudes
}

\author{
Teresa de la Hera \& Joost Raessens
}

\begin{abstract}
Can digital games be used for persuasion? The results of studies that have tried to validate the persuasive potential of digital games seem to be inconclusive. This is because there are multiple ways and strategies to persuade players through games, and some of these may work to address specific goals while others do not. So, the question is not if digital games can be used to persuade players but how these games can be efficiently designed to intentionally change attitudes. To better explain how digital games can be efficiently designed for persuasion, we will refer to the shortcomings mentioned by Sicart when arguing against procedurality and how we believe these flaws can be addressed to ensure the persuasive efficiency of digital games.
\end{abstract}

Keywords: persuasive games; persuasion; procedural rhetoric; attitude change

\section{Introduction}

The academic debate on the persuasive potential of digital games culminated in 2011 when Miguel Sicart published his paper 'Against Procedurality'. In it, Sicart argued against Ian Bogost's claim (2007) about the unique persuasive potential of digital games. His discourse was focused on countering Bogost's theory on procedural rhetoric, that is, on the exclusive capacity of digital games to persuade through rule-based representations. Bogost claimed that

Hera, T. Dela, J. Jansz, J. Raessens, B. Schouten, Persuasive Gaming in Context. Amsterdam: Amsterdam University Press, 2021 DOI 10.5117/9789463728805_CHO4 
the ability of digital games to explain processes through interactions was a powerful persuasive characteristic, making them unique when it came to persuading players.

Sicart, however, positioned himself against this theory, stating that Bogost was ignoring the creativity of players and the fact that they tend to appropriate game rules (Sicart, 2011), which does not guarantee the transmission of persuasive messages in the way intended by designers. According to Sicart (2011), Bogost was disregarding the fact that players look for a sense of agency, that is, having the perception that different actions in a game result in different game experiences, and that, by extension, they as players have control over what happens in the game through their own performance. From Sicart's perspective, persuasive games designed using a procedural rhetoric approach would be too restricted, limiting players' sense of agency and therefore becoming unattractive to play.

Sicart was not, however, the only one criticizing Bogost's claims. Other authors such as Heide and Nørholm (2009), Ferrari (2010), and Antle (2014) have also argued against Bogost's theory. In addition, Bogost himself, thirteen years after publishing his book on persuasive games, has admitted being wrong in some of his predictions related to their persuasive potential (see Chapter 1 of this volume). Does this mean that digital games cannot be used for persuasion? More precisely, does this mean that digital games cannot be designed to intentionally change the attitudes or behavior of a player?

\section{Can digital games be used for persuasion?}

To answer this question, we also need to answer another: are digital games persuasive in the first place? By this, we mean: do digital games have the capacity to influence the attitudes or behavior of players, whether intentionally or not? Scholars (Flanagan, 2010; Flanagan \& Nissenbaum, 2014; Frasca, 2001; Grace, 2009; Salen \& Zimmerman, 2005; Walz, 2003) seem to agree that the answer is yes: digital games are persuasive.

As cultural artifacts, digital games are full of meaning and, whether or not they are designed with persuasive intentions, they always convey a message that can be interpreted by their players. Depending on players' personal circumstances and the context in which the game is being played, the game will, in one way or another, influence attitudes toward the topic the game is covering (Grace, 2009; Murray, 1999; Salen \& Zimmerman, 2005). If this is true and digital games are persuasive, it seems logical to conclude that they can be used for persuasion, i.e., to intentionally influence the attitudes or 
behavior of players. Scholars do not, however, seem to agree on this. What is unclear, though, is if it is possible to design an appealing digital game for players that can at the same time successfully affect their attitudes in the way intended by designers.

If we pay attention to the results of studies on the effectiveness of persuasive games, providing an answer to this question becomes even more complicated: the results of studies that have tried to validate the persuasive potential of digital games seem to be inconclusive (for a full overview of these studies, read Chapter 10 of this volume). We use the word 'seem' deliberately here, because if we compare the results of different studies we may form the impression that they have contradictory results (Jacobs, 2017; Jacobs, Kneer, \& Jansz, 2019; van 't Riet, Meeuwes, van der Voorden, \& Jansz, 2018a). While some research concludes that persuasive games do indeed work to persuade players in the way intended by designers (e.g., Kampf \& Cuhadar, 2015; Peng, Lee, \& Heeter, 2010; Ruggiero, 2015), other scholars conclude that not all persuasive games are effective in the way intended, or, at least, they are no more effective than traditional media forms (e.g., Van't Riet, Meeuwes, Van der Voorden, \& Jansz, 2018b; Chapter 12), unlike Bogost's original claim.

We do not intend to carry out a detailed analysis of the studies conducted on the effectiveness of persuasive games, as this has already been done by Jacobs and Jansz in Chapter 10. If we raise this question here, it is to make the claim that the main reason for these apparently inconsistent results is that persuasive games can be: used for a great variety of purposes; aimed at changing the attitudes of many different types of target group; and applied in disparate contexts. This means that there are multiple ways and strategies to persuade players through games, and some of these may work to address specific goals while others do not. So, the question is not if digital games can be used to persuade players but how these games can be efficiently designed to intentionally change attitudes. Note that we are focusing my attention on how these games can be 'efficiently designed' and not how they can be 'designed to effectively change the attitudes of players'. This is because the effects of persuasive games depend on many factors besides the design of the game itself-effects that cannot be completely controlled by designers (e.g., the context in which the game is played, the mood of the player when playing the game). We want to argue, however, that some design decisions can be consciously made by paying attention to specific persuasive intentions and goals, which can improve the efficiency of a persuasive game.

To better explain how digital games can be efficiently designed for persuasion, we will refer to the shortcomings mentioned by Sicart when 
arguing against procedurality and how we believe these flaws can be addressed to ensure the persuasive efficiency of digital games. In the next two sections, we will therefore answer the following questions: How can digital games give freedom to players and secure persuasion at the same time? And how can digital games be engaging and persuasive concurrently?

\section{Giving freedom, securing persuasion. Persuasion through digital games goes beyond procedural rhetoric}

One of the shortcomings in Bogost's approach (2007) identified by Sicart (2011) concerned how, from the latter's perspective, building persuasion based on procedural rhetoric would mean limiting players' freedom in the game and, by extension, their sense of agency. Efficiently designing games that meet both requirements - i.e., giving freedom to players and meeting a concrete, persuasive goal-requires a good understanding of how digital games can be used to persuade (De la Hera, 2013). In this regard, we have a different vision to Bogost with respect to the ways in which digital games can be used for persuasion. Our main claim, which coincides with the arguments of other scholars in this field such as Heide and Nørholm (2009) and Nelson (2012), is that digital games have a unique potential to persuade players, but this potential includes — but is not limited to-procedural rhetoric. We contend that other persuasive dimensions of digital games should be taken into consideration, and these dimensions are what make digital games interesting persuasive content.

Teresa de la Hera (2019) proposed a theoretical model that goes beyond procedural rhetoric to explain how digital games persuade players. With this model, De la Hera explains that it is not only the rules of the game that convey meaning but that it is also important to acknowledge that there are other elements in a game that are also relevant to persuasion (De la Hera, 2019). Using this model, the author explains how other elements in a game - such as the visuals, the sound, the story, or the audiovisual treatment-can serve to influence how the content of the game is interpreted by players (2019). The model does more than reflect how elements in the game can be used for persuasion; it also explains strategies that can be used in a game's design to persuade players, such as delivering pleasurable sensorial experiences (e.g., using nice vs. irritating background music), fostering social interactions, or appealing to emotions like fear or happiness. All these persuasive dimensions, explained in much more detail in De la 
Hera's book Digital Gaming and the Advertising Landscape (2019), can be used to persuade a player.

At this point, it is important for us to highlight that our approach is slightly different from the one that Sebastian Deterding (2016) defends. Deterding supports the idea that other persuasive dimensions, such as visual or narrative persuasion, help to frame procedural rhetoric; that is, Deterding still defends Bogost's vision that procedural rhetoric is the backbone of persuasion in digital games, although his discourse supports the idea that persuasion through these games is not reduced to procedural rhetoric and that other dimensions serve to frame this expression. In other words, they add meaning to what is being conveyed through procedural rhetoric. Our perspective on this is different. We contend that these other persuasive dimensions can persuade independently from procedural rhetoric (we give examples of this in the following sections). In other words, procedural rhetoric is only one of the persuasive dimensions that can be used in digital games and is not the persuasive dimension for excellence. For this reason, persuasion through digital games can be achieved through a combination of one of more of these dimensions (including — or not including — procedural rhetoric).

The fact that other persuasive dimensions, such as narrative, sensorial, or sonic persuasion, can be used to influence players' attitudes through digital games helps to support the idea that persuasive games can be open to letting their players feel the sense of agency they require in order to be engaged in the experience and still convey a message that is aligned with the designers' goals (De la Hera, 2019). We support this claim because, if persuasion through digital games is only based on and limited to procedural rhetoric, this means that attempting to persuade players is unnecessary when designing games in which we can predict their performances and so ensure that they experience the game in the manner expected and the persuasive message is conveyed. If persuasion through digital games is the result of a combination of multiple persuasive dimensions, as De la Hera (2019) contends, digital games offer many more possibilities and flexibility for persuasion than procedural rhetoric suggests, allowing for the design of less restricted games than envisioned by those who are against the procedural rhetoric school.

\section{Balancing engagement and persuasion: aligning persuasive goals and game goals}

As we support the idea that persuasion through digital games is not only reduced to procedural rhetoric and that the persuasive options are multiple, 
the following question arises: How can we design persuasive games efficiently? That is: How should we decide which persuasive dimensions to use and how should we use them to identify the right balance between engagement and persuasion?

We know that persuasive games can be applied to a great variety of persuasive goals that can be aimed at multiple target groups and can be played in different contexts and situations (Jacobs, Jansz, \& De la Hera, 2017). For example, we can talk about games that aim to change eating habits (Orji, Mandryk, Vassileva, \& Gerling, 2013); influence children's attitudes toward sports (Staiano \& Calvert, 2011); foster empathy toward refugees (Kors, Ferri, van der Spek, Ketel, \& Schouten, 2016; Raessens, 2010); change players' views in relation to climate change (Raessens, 2018, 2019a); or foster multicultural or intergenerational interactions (Alencar \& De la Hera, 2018; De la Hera, Loos, Simons, \& Bloom, 2017; Loos, De la Hera, Simons, \& Gevers, 2019). We can also talk about mobile games (Winter et al., 2011), online games (Wen, Kow, \& Chen, 2011), virtual reality games (Raessens, 2019b; Reid, 2002), and pervasive games (Walz \& Ballagas, 2007).

Although some scholars in the field of persuasive games have been trying to propose models and frameworks that help us to better understand how to approach the process of persuasive game design (e.g., Kors, Spek, \& Schouten, 2015; Siriaraya, Visch, Vermeeren, \& Bas, 2018), it is almost impossible to come up with a magic formula, or even a magic cookbook, for the design of persuasive games due to the wide diversity of applications and solutions. The cookbook proposed by Siriaraya and colleagues (2018), for example, which is the most complete design approach for persuasive games published to date, includes a detailed list of steps and elements to consider during the design process but still lacks clear directions on how to make design decisions. This is the result, as previously stated, of the complexity of digital games as persuasive contents and their multiple persuasive applications.

That being said, there are still some aspects that could be taken into account when making design decisions about persuasive games. In this chapter, we want to focus on two relevant factors that should be taken into consideration when making decisions about which persuasive strategy to adopt: the level of resistance of players toward persuasion and the prior knowledge of players about the topic covered in the game.

When considering the different levels of player resistance and prior knowledge of the topic being covered in a game, there are three different persuasive goals that games may try to achieve: shaping, reinforcing, or changing the attitudes of players. Our claim is that the design approach should be different depending on the main persuasive goal of the game. 
This is a complex matter in which multiple elements need to be taken into account. In this section, however, our focus is on how different approaches in the relationship between game goals and persuasive goals should be considered.

\section{Shaping new attitudes}

When the target audience of a persuasive game does not have prior knowledge of the topic being covered, the main persuasive purpose is usually to shape a new attitude. In this case, we are normally speaking about players who do not have firm beliefs or established preferences in relation to the subject matter; that is, the game does not face high levels of player resistance toward persuasion. In these cases, the main purpose of the game is typically to convey information that can be useful for a player when it comes to better understanding the question being addressed in the game. When a new topic is introduced and there is no major resistance to overcome, we can design games in which the game goal and the persuasive goal overlap. In games where this occurs, players need to demonstrate that they understand the persuasive message if they are to be successful and progress in the game (Heide \& Nørholm, 2009).

An example of a persuasive game that responds to the need to shape a new attitude is Plague Inc. (Ndemic Creations, 2012). The persuasive goal of this game is to help players understand the different ways in which diseases can spread depending on environmental factors and specific countries' economic statuses. This persuasive goal is clearly linked to the game goal of players, who have to evolve a pathogen present in a selected country from a non-lethal disease into a highly infectious epidemic capable of ending life as we know it. This means that in order to win the game, players need to understand how diseases spread and how this process is accelerated. Depending on the strategy a player decides to adopt, they will be able to achieve their final goal. In this way, they can learn about and understand the reasons why less developed countries have a harder time fighting a disease while richer countries scramble to find a cure.

In this example, the game goal and the persuasive goal are perfectly aligned. These games take the form of a simulation in which the player confronts situations in the game in the same way that this happens in real life. This is a way for players to understand the causes and effects of specific situations. It is also a good approach to introducing players to new topics - or to more detailed insights into subjects about which they are already aware-in an environment in which they can experience these topics 
in the first person. This is the way Bogost (2007) imagined that persuasive games should work. In his view, such games should serve as simulations in which players can explore first-hand the consequences of their decisions or behaviors, and, as we see in the examples described, procedural rhetoric can serve as the backbone for persuasion.

It is probably easy for the reader to recognize the value of this approach. When the game goal and persuasive goal are aligned, it is clear that players need to show that they understand the message conveyed if they are to succeed in the game. Our point here is that these approaches are only successful when we are trying to introduce players to something about which they have no prior knowledge and that deals with persuasive messages to which they do not have any resistance. If players do have prior knowledge of the topic the game covers, it is reasonable to state that, if they are experiencing a simulation of something they fully understand, the causes and effects would not be especially attractive. Furthermore, and more importantly, if as well as having prior knowledge of the persuasive message players also have a resistance to being persuaded, it can also be the case that, in simulations like this one, they pretend to agree with the message in order to win the game. This clearly does not necessarily mean that they have changed their attitude to the topic the game covers.

\section{Reinforcing existing attitudes}

The second case concerns persuasive games that do not need to overcome players' resistance to persuasion. This is because their main purpose is to reinforce attitudes that are already aligned with the idea the game is trying to convey, although they still need to deal with the fact that players already have prior knowledge of the topic that the game covers. In this group of games, we want to highlight those whose main purpose is to support the player who is convinced that a specific behavior or habit is positive for his/her life but who needs extra support or motivation to engage in this habit or behavior.

The best option for games that aim to reinforce existing attitudes is to design a game in which the game goal and the persuasive goal are somehow related to each other, even though they do not overlap. An example of how this can be achieved is the game Papo \& Yo (Minority Media, 2012), a $3 \mathrm{D}$ puzzle-platformer driven by a story that allegorizes what it means for a child to grow up with an alcoholic father. The persuasive goal of this game is to help the player understand that there is nothing a child can do alone to prevent the damage an alcoholic parent can cause and to demonstrate the relevance of seeking help in situations like this. 
The game goal in this case is slightly different but is still connected with the persuasive goal. In the game, the player guides a young boy, Quico, on his quest to find a cure for his best friend, Monster, a gentle, fruit-consuming giant that transforms into a recklessly violent behemoth after he consumes his favorite snack, frogs. The player will find that, no matter what Quico does when Monster enters a frog-induced rage, he will never be able to fight back. The only thing that will work for him is to run and hide and to try to overcome the obstacles blocking his path. The ending of this game is a sequence that teaches Quico that he needs help to fight Monster's addiction, as this is something he cannot do alone. Quico receives help from Lula, a robot that allows him to jump across gaps that are otherwise too wide for him to overcome, and Alejandra, a girl who provides guidance on his journey.

This is a game that addresses a difficult and sensitive topic. In this case, the persuasive goal is to reinforce the idea that there is not much a child can do on their own to fight the terrible damage that an alcoholic parent can cause to a family. Although the players of this game probably understand the relevance of seeking help, anyone who has faced a similar situation knows how hard it is to make a decision to actually ask for support. Children of alcoholic parents usually feel ashamed and even guilty about their situation, and it is common for them to try to hide what they are experiencing. As a result, it is very difficult to decide to look for help or support.

This game is an allegory of this difficult topic, in which narrative persuasion is used to transform the persuasive goal into a game goal that helps the player to reflect on their terrible circumstances. So, we have a game goal that does not completely overlap with a persuasive goal but is connected to it. In this case, procedural persuasion is being used but is clearly framed by narrative persuasion. We can see here how narrative persuasion not only frames procedural persuasion but also completely transforms the players' experience. Accordingly, this game goes beyond the idea of persuasive games being simulations in which the player can experience in the first person how specific performances or choices have concrete consequences. Narrative persuasion is used to give the player the opportunity to look at this issue through a different lens, perhaps relieving part of the emotional overload attached to the real experience.

This is the way Sebastian Deterding (2016) envisions how digital games can be used for persuasion. From his perspective, procedural rhetoric is still the backbone of the persuasive experience, while other persuasive dimensions, such as narrative or visual persuasion, can be used to frame how the message conveyed through procedural rhetoric is interpreted and perceived by players. In the next section, however, we will try to argue how 
persuasion through digital games can also work without procedural rhetoric being the backbone of persuasion, and how other persuasive dimensions can also take on the main role when trying to persuade players.

\section{Changing attitudes}

In persuasive games whose purpose is to change players' attitudes, the overlap between the persuasive goal and the game goal does not work in the same way as it does for games that aim to shape or reinforce an existing attitude. Overcoming informed players' resistance to persuasion is challenging. In this case, we are talking about players who have prior knowledge about the topic the game covers and who have an attitude that is contrary to the one the game is trying to promote. In this case, designing a simulation game in which the player needs to understand the message that the game is conveying in order to win is, in some circumstances, pointless because informed players may make the 'right' choices in the game and pretend to agree with its point in order to win without actually changing their beliefs or actions in the physical world. In this case, a game in which the game goal and the persuasive goal differ completely would better fit the purposes of the game.

We will start with an example of a game that tries to change the attitude of players but still has a game design in which the game goal and the persuasive goal overlap. The game I want to discuss here is Against All Odds (UNHCR, 2006), which was developed for the UNHCR with the aim of promoting empathy and positive attitudes toward refugees by putting players in their shoes. Although it is true that this game is available online and could have been played by a broad audience, ranging from players who already empathize with refugees to those who do not, its main purpose is still to promote positive attitudes and empathy among players who are not particularly sympathetic toward refugees.

We want to use Against All Odds as an example here because previous quantitative studies on the effectiveness of this game have concluded that it is unsuccessful in promoting long-lasting attitude changes when compared to traditional forms of media (Van 't Riet, Meeuwes, Van der Voorden, \& Jansz, 2018; Wertley chapter 12 of this volume). In addition, a qualitative study (Domalewska, 2018) conducted to understand how the game was perceived and interpreted by its players has concluded that, although players understand the message that the UNHCR is trying to convey, they do not seem to personally empathize or feel real emotions toward refugees while playing the game. A majority of participants in this qualitative study were also critical of the way 
in which the game presents refugees' journeys, and most of them also claimed that the game was unhelpful in terms of making them reflect seriously on the refugees' experiences, especially in relation to what they have to deal with and the losses and dangers they face when moving to another country.

If we analyze the persuasive strategy of this game, we can see that its game goal and persuasive goal overlap. In order to win the game, players need to adopt the role of a refugee in a simulation experience in which they will flee a country of conflict to a new country. To succeed in this journey, players will need to make a series of decisions, such as leaving their country, avoiding being seen by the police and the military, and giving answers in an interrogation.

When explaining why they did not empathize with the refugees in this game, the participants referred to the fact that they already had an opinion about this issue before playing the game, suggesting that it did not succeed in changing their opinions. Furthermore, the players also referred to the fact that the game was leading them to the choices they were supposed to make in the game, with many stating that they were actually not making these decisions voluntarily but rather because they needed to make them to continue playing.

When the purpose of a game is to change the attitude of a player, a different game design approach is one in which the game goal and the persuasive goal are completely different. A good example of this is the game SnowWorld (University of Washington Harborview Burn Centre in Seattle, 2011). The persuasive goal of this game is to change the attitude of patients with severe burns toward their rehabilitation and wound care, which can be very painful and stressful. The game was designed based on the scientific claim that context and distractions can influence the way individuals experience pain. SnowWorld is a virtual reality game in which the player is immersed in a snowy forest where the game goal is to fight a snowman. The snowman throws snowballs at the player, who needs to throw them back to hit his/ her assailant. The player also faces penguins that have to be destroyed. The player can control the game with the movement of his/her head, and so the rest of the body is free for rehabilitation and wound care.

The game has been designed in such a way that players are focused on the experience and the feelings triggered by it, which distracts them from the reality of their treatment. In this case, the persuasive strategies are used to keep players busy and motivated enough in the game, which relieves some of the stress arising from the difficult process they are experiencing. Tactical and sensorial persuasion is, in this case, the most relevant persuasive dimension used. Sensorial persuasion consists of designing an experience that engages players through the senses. In this case, they are immersed in an environment of ice and snow to help them to get through their terrible 
treatment. Tactical persuasion, meanwhile, consists of using the rules of a game to create an experience that is challenging enough for players but, at the same time, is adapted to their skills so that they do not become frustrated. The game is deliberately simple, because patients in pain cannot focus on complex mechanics, but it is intensive and demanding enough to keep them engaged. In this case, the game goal and persuasive goal do not overlap.

This is also an example of how a persuasive game can be designed without procedural rhetoric being the main persuasive dimension used in the game. We can see in this case how other persuasive dimensions are more relevant and are put in the service of a persuasive goal. In this case, a persuasive game designed to simulate the experience of going through a healing process would not have helped to achieve the persuasive goal of the designers.

\section{Conclusion}

In this chapter, we have argued how it is possible to look beyond procedural persuasion in digital games, that is, beyond the use of rule-based representations to influence the attitudes of players. Our main point is that procedural persuasion is one of the persuasive dimensions that can be used within digital games, although it is not the most relevant dimension, contrary to the position defended by Bogost in his book Persuasive Games. We have argued that persuasive goals and game goals could be aligned in different ways depending on whether the main purpose of the game is to shape, reinforce, or change the attitudes of players. We have illustrated this with different examples, with the aim being to defend the idea that if we pay attention to players' prior knowledge of the persuasive goal and their resistance to persuasion, we can design games that are efficient in terms of being interesting and attractive for players to play but still achieve their persuasive goals. This approach broadens the understanding of what persuasive games are and how they can be used for persuasion. It is also much more flexible than the position defended by Bogost; it challenges the shortcomings identified by Sicart; and it is used to argue against procedural rhetoric and, by extension, the persuasive potential of digital games.

\section{References}

Alencar, A. \& De la Hera, T. (2018). Gaming in Multicultural Classrooms: The Potential of Collaborative Digital Games to Foster Intercultural Interaction. In 
Social Interactions in Virtual Worlds, pp. 288-310. Cambridge, UK: Cambridge University Press. https://doi.org/10.1017/9781316422823.012.

Antle, A.N., Tanenbaum, J., Macaranas, A., \& Robinson, J. (2014). Games for Change: Looking at Models of Persuasion Through the Lens of Design. In A. Nijholt (ed.), Playful User Interfaces. Singapore: Springer Singapore. https://doi.org/10.1007/978981-456o-96-2_8.

Bogost, I. (2007). Persuasive Games: The Expressive Power of Videogames. Cambridge, MA: MIT Press.

De la Hera, T. (2013). A Conceptual Model for the Study of Persuasive Games. Proceedings of DiGRA 2013: DeFragging Game Studies, 1-15. Retrieved 11 December 2018 from http://www.digra.org/digital-library/publications/a-conceptual-modelfor-the-study-of-persuasive-games/.

. (2019). Digital Gaming and the Advertising Landscape. Amsterdam: Amsterdam University Press.

., Loos, E., Simons, M., \& Bloom, J. (2017). Benefits and Factors Influencing the Design of Intergenerational Digital Games: A Systematic Literature Review. Societies, 7(3), 1-15. https://doi.org/10.3390/soc7030018.

Deterding, S. (2016). The Mechanic is not the (Whole) Message: Procedural Rhetoric Meets Framing in Train \& Playing History 2. 1st International Joint Conference of DiGRA and FDG. Dundee, Ireland. Retrieved 4 December 2018, from https:// mechanicmessage.wordpress.com/.

Domalewska, A. (2018). Persuasive Communication within the Game Against All Odds [Unpublished MA Thesis]. Erasmus University Rotterdam.

Ferrari, S. (2010). The Judgment of Procedural Rhetoric [PhD Thesis]. Georgia Institute of Technology, Georgia. Retrieved 11 May 2018 from https://smartech.gatech. edu/bitstream/handle/1853/33915/ferrari_simon_f_201005_mast.pdf.

Flanagan, M. (2010). Creating Critical Play. In R. Catlow, M. Garrett, \& C. Morgana (eds.), Artists Re:thinking Games, pp. 49-53. Liverpool: Liverpool University Press.

—_, \& Nissenbaum, H. (2014). Values at Play in Digital Games. Cambridge, MA: MIT Press. https://doi.org/10.1177/1461444816631742.

Frasca, G. (2001). Videogames of the Oppressed: Videogames as a Means for Critical Thinking and Debate [MA Thesis]. Georgia Institute of Technology, Georgia. Retrieved 15 May 2018 from https://ludology.typepad.com/weblog/articles/ thesis/FrascaThesisVideogames.pdf.

Grace, L. (2009). The Philosophies of Software. In J. Braman, G. Vincenti and G. Trajvokski (eds.), Handbook of Research on Computational Arts and Creative Informatics, pp. 326-342. https://doi.org/10.4018/978-1-60566-352-4.

Heide, J., \& Nørholm, S. (2009). Playful Persuasion. The Rhetorical Potential of Advergames. Nordicom Review, 3o(2), 53-68. 
Jacobs, R.S. (2017). Playing to Win over: Validating Persuasive Games [PhD Thesis]. ERMeCC-Erasmus Research Center for Media Communication and Culture. Retrieved 12 October 2019 from https://repub.eur.nl/pub/102769/.

., Jansz, J., \& De la Hera, T. (2017). The key features of persuasive games: A model and case analysis. In T. Quandt \& R. Kowert (eds.), New Perspectives on the Social Aspects of Digital Gaming: Multiplayer 2, pp. 153-171. Oxford: Routledge. https://doi.org/10.4324/9781315629308.

., Kneer, J., \& Jansz, J. (2019). Playing against abuse: Effects of Procedural and Narrative Persuasive Games. Journal of Games, Self, and Society, 1(1), 97-120. https://doi.org/10.1184/R1/7857578.

Kampf, R., \& Cuhadar, E. (2015). Do Computer Games Enhance Learning About Conflicts? A Cross-National Inquiry Into Proximate and Distant Scenarios in Global Conflicts. Computers in Human Behavior, 52 (Suplement C), 541-549. https://doi.org/10.1016/j.chb.2014.08.008

Kors, M.J.L., Ferri, G., van der Spek, E.D., Ketel, C., \& Schouten, B.A.M. (2016). A Breathtaking Journey: On the Design of an Empathy-Arousing Mixed-Reality Game. Proceedings of the 2016 Annual Symposium on Computer-Human Interaction in Play - CHI PLAY'16. https://doi.org/10.1145/2967934.2968110.

Kors, M.J.L., Spek, E. Van Der, \& Schouten, B. (2015). A Foundation for the Persuasive Gameplay Experience. Proceedings of the 1oth International Conference on the Foundations of Digital Games. Retreived 20 May 2018 from https:// research.tue.nl/en/publications/a-foundation-for-the-persuasive-gameplayexperience.

Loos, E., De la Hera, T., Simons, M., \& Gevers, D. (2019). Setting Up and Conducting the Co-design of an Intergenerational Digital Game: A State-of-the-Art Literature Review. In J. Zhou \& G. Salvendy (eds.), Human Aspects of IT for the Aged Population. Design for the Elderly and Technology Acceptance, pp. 56-69. Cham: Springer International Publishing.

Minority Media. (2012). Papo \& Yo [Digital Game].

Murray, J.H. (1999). Hamlet on the Holodeck. New York, NY: Free Press.

Ndemic Creations. (2012). Plague Inc.[Digital Game].

Nelson, M.J. (2012). Sicart's “Against Procedurality”. Retrieved 23 August 2018, from http://www.kmjn.org/notes/sicart_against_proceduralism.html.

Orji, R., Mandryk, R.L., Vassileva, J., \& Gerling, K.M. (2013). Tailoring Persuasive Health Games to Gamer Type. In Proceedings of the SIGCHI Conference on Human Factors in Computing Systems - CHI '13 (p. 2467). https://doi. org/10.1145/2470654.2481341.

Peng, W., Lee, M., \& Heeter, C. (2010). The Effects of a Serious Game on Role-Taking and Willingness to Help.Journal of Communication, 6o(4), 723-742. https://doi. org/10.1111/j.1460-2466.2010.01511.x. 
Raessens, J. (2010). A Taste of Life as a Refugee: How Serious Games Frame Refugee Issues. Changes in Museum Practice: New Media, Refugees and Participation., (2005), 94-105. Oxford: Berghahn.

— . (2018). Ecogames: Playing to save the planet. In T. Meiris \& G. Rippl (eds.), Cultural Sustainability: Perspectives from the Humanities and the Social Sciences. London and New York: Routledge.

- (2019a). Collapsus, or How to Make Players Become Ecological Citizens. In R. Glas, S. Lammes, M. de Lange, J. Raessens, \& I. de Vries (eds.), The Playful Citizen. Civic Engagement in a Mediatized Culture. Amsterdam: Amsterdam University Press.

— . (2019b). Virtually Present, Physically Invisible: Alejandro G. Iñárritu's Mixed Reality Installation Carne y Arena. Television \& New Media, 2o(6), 634-648. https://doi.org/10.1177/1527476419857696.

Reid, D.T. (2002). Benefits of a Virtual Play Rehabilitation Environment for Children With Cerebral Palsy on Perceptions of Self-Efficacy: A Pilot Study. Pediatric Rehabilitation, 5(3), 141-148. https://doi.org/10.1080/1363849021000039344.

Ruggiero, D. (2015). The Effect of a Persuasive Social Impact Game on Affective Learning and Attitude. Computers in Human Behavior, 45, 213-221. https://doi. org/10.1016/j.chb.2014.11.062.

Salen, K., \& Zimmerman, E. (2005). Game Design and Meaningful Play. In J. Raessens \& J.H. Goldstein (eds.), Handbook of Computer Game Studies, pp. 59-79. Cambridge, MA: MIT Press.

Sicart, M. (2011). Against Procedurality. Game Studies, n(3). Retrieved 7 May 2018 from http://gamestudies.org/1103/articles/sicart_ap.

Siriaraya, P., Visch, V., Vermeeren, A., \& Bas, M. (2018). A Cookbook Method for Persuasive Game Design. International Journal of Serious Games, 5(1). https:// doi.org/10.17083/ijsg.v5i1.159.

Staiano, A.E., \& Calvert, S.L. (2011). Exergames for Physical Education Courses: Physical, Social, and Cognitive Benefits. Child Development Perspectives, 5(2), 93-98. https://doi.org/10.1111/j.1750-8606.2011.00162.x.

UNHCR. (2006). Against all odds [Digital Game].

University of Washington Harborview Burn Centre in Seattle (2011). SnowWorld [Digital Game].

Van 't Riet, J., Meeuwes, A.C., van der Voorden, L., \& Jansz, J. (2018a). Investigating the Effects of a Persuasive Digital Game on Immersion, Identification, and Willingness to Help. Basic and Applied Social Psychology, 4o(4), 180-194. https:// doi.org/10.1080/01973533.2018.1459301.

. (2018b). Investigating the Effects of a Persuasive Digital Game on Immersion, Identification, and Willingness to Help. Basic and Applied Social Psychology, pp. 1-15. https://doi.org/10.108o/o1973533.2018.1459301. 
Walz, S.P. (2003). Delightful Identification \& Persuasion: Towards an Analytical and Applied Rhetoric of Digital Games. In M. Copier \& J. Raessens (eds.), Level Up. Digital Games Research Conference. Utrecht: Utrecht University.

__., \& Ballagas, R. (2007). Pervasive Persuasive: A Rhetorical Design Approach to a Location-Based Spell-Casting Game for Tourists. Situated Play, Proceedings of DiGRA 2007 Conference, 489-497. Retrieved from http://www.digra.org/dl/ db/07311.17007.pdf.

Wen, J., Kow, Y.M., \& Chen, Y. (2011). Online Games and Family Ties: Influences of Social Networking Game on Family Relationship. In Lecture Notes in Computer Science (including subseries Lecture Notes in Artificial Intelligence and Lecture Notes in Bioinformatics) (Vol. 6948 LNCS, pp. 250-264). https://doi. org/10.1007/978-3-642-23765-2_18.

Winter, S., Richter, K.-F., Baldwin, T., Cavedon, L., Stirling, L., Duckham, M., Kealy, A., Rajabifard, A. (2011). Location-Based Mobile Games for Spatial Knowledge Acquisition. In K. Janowicz (Ed.), Cognitive Engineering for Mobile GIS. Belfast. Retrieved ${ }_{15}$ September 2018 from http://people.eng.unimelb.edu.au/winter/ pubs/winterullocation.pdf.

\section{About the authors}

Teresa de la Hera is Assistant Professor of Persuasive Gaming at Erasmus University Rotterdam, where she is a member of the Research Cluster Gaming Matters. Her expertise is focused on understanding how digital games are used as media for communication and tools for engagement, motivation, and persuasion. She is the author of Digital Gaming and the Advertising Landscape (2019), published by Amsterdam University Press.

Joost Raessens is Chair and Full Professor of Media Theory at Utrecht University and scientific director of the Utrecht Center for Game Research. He is co-editor of the volumes The Playful Citizen (2019) and Playful Identities (2015), both published by Amsterdam University Press, and the Handbook of Computer Game Studies (The MIT Press, 2005). 


\title{
5. Creating Stealth Game Interventions for Attitude and Behavior Change: An 'Embedded Design' Model
}

\author{
Geoff Kaufman, Mary Flanagan \& Max Seidman
}

\begin{abstract}
The chapter will open with examples of transformational games that utilize overt, explicit approaches to attitude and behavior change. While acknowledging the worthwhile intentions of such games and their potential utility for triggering reflection and action, this overview will present the central premise of the chapter: that there are a number of fundamental reasons why explicit approaches can backfire or be of limited utility for persuasion and that the use of more implicit, covert approaches to persuasion can be more effective. The 'embedded design' model presented in this chapter is particularly relevant for games attempting to engage players with sensitive or potentially threatening topics or to address attitudes or behaviors that themselves are implicit or unconscious.
\end{abstract}

Keywords: embedded design; persuasive games; stealth design; attitude change

\section{Introduction}

The past decade has seen the emergence of a plethora of persuasive games that aim to increase players' awareness of critical and timely social issues - and to change players' attitudes and behaviors - through gameplay (Bogost, 2007). Running the gamut from games targeting cognitive biases that reduce the accuracy of judgment and decision-making (e.g., the SIRIUS initiative of the Intelligence Advanced Research Projects Activity program, see Dunbar et al., 2013) to ones intended to encourage behaviors that benefit

Hera, T. Dela, J. Jansz, J. Raessens, B. Schouten, Persuasive Gaming in Context. Amsterdam: Amsterdam University Press, 2021 DOI 10.5117/9789463728805_CHO5 
society (such as recycling in the case of the mobile game Gaea, as studied by Centieiro, Romão, \& Dias, 2011, or the promotion of positive attitudes and altruistic behaviors toward refugees, the focus of Van 't Riet, Meeuwes, Van der Voorden \& Jansz, 2018) or the self (e.g., the reduction of substance abuse and HIV risk, which is the focus of the 'Play2Prevent' program, see Fiellin et al., 2014), this subset of 'serious games' is united by their intention to transform mindsets and actions through the messages they model.

Games themselves are powerful sites for enculturation (Flanagan, 2009). A vast majority of serious games, however, share a common design philosophy: by and large, they present characters, scenarios, situations, and solutions in a direct, matter-of-fact fashion under the ostensibly logical (and wellintentioned) assumption that doing so will automatically encourage and enable players to internalize, and transfer, the game's modeled beliefs and behaviors to real-life contexts.

In light of what is known in contemporary psychology, this approach, we argue, is ill-advised at best and potentially harmful at worst, particularly when dealing with persuasive content that is uncomfortable, psychologically threatening, or counter-attitudinal. A vast body of social psychological theory and research on persuasion and attitude change has long demonstrated that it is a basic human tendency to resist persuasive communications that are perceived as too forceful or forthright in their intentions. For one, being aware that some external agent is aiming to change one's attitudes or behaviors triggers psychological reactance: an aversive state of arousal that arises whenever one perceives that his/her freedom to do or think freely is being threatened (Brehm, 1966). The aversive state of reactance raises individuals' psychological defenses, rendering them less receptive (and, indeed, more resistant) to a persuasive message. What's even more surprising is that psychological reactance will occur even if a person's own beliefs align with the content of the message (e.g., Worchel \& Brehm, 1970).

A second psychological barrier that comes into play in situations of persuasion and play, especially when dealing with attitudes and behaviors of a particularly sensitive nature (such as the hot-button issues of stereotypes and prejudice), is the bias blind spot: the acknowledgment that biases exist but the denial or minimization of one's own susceptibility to those biases (e.g., Pronin, Lin, \& Ross, 2002).

The potentially aversive and defensive reactions triggered by explicit persuasive attempts limit not only the potential efficacy of game-based interventions but also players' enjoyment of them, for the perception of a persuasive agenda is inherently antithetical to players' immersion within a game world (and, indeed, antithetical to the notion of play itself: see de la 
Hera Conde-Pumpido, 2013; Huizinga, 1938). In other words, most persuasive games may fail to engage players or are unable to immerse players in a transformative experience, due to normal psychological human reactions to overtly 'message-driven' interventions.

For this reason, we propose that persuasive games would greatly benefit from using a subtler, stealthier approach to presenting their focal messages or themes. This line of thinking has led us to develop and test novel strategies for persuasion via games or stories (including those discussed in this chapter) to address controversial topics such as public health attitudes and social and cognitive biases in a more nuanced, less direct fashion. In this chapter, we describe our model of 'Embedded Design' that offers key strategies for tackling social issues and including persuasive content in a game in ways that circumvent players' psychological defenses, trigger a more receptive mindset for internalizing the game's intended message, and do so without sacrificing players' enjoyment or the game's replayability.

\section{The 'embedded design' model}

The key premise of the Embedded Design model is that the persuasive impact of game-based interventions is greatly enhanced when interweaving a focal message within the game's content, mechanics, or context of play rather than making the message or the game's persuasive aims the focal point. Through our team's longstanding work in the design and study of games intended to shift attitudes and behaviors, we have uncovered a number of distinct embedding strategies that have proven effective at increasing our games' persuasive impact (see Figure 5.1). This work is informed in part by the Values in Design and the Values at Play methodology, which offers many avenues at which values might emerge in any given game experience (see Flanagan \& Nissenbaum, 2014).

Here, we will focus primarily on three distinct strategies - each representing a unique manifestation of Embedded Design - that have emerged thus far in our work: (1) Intermixing: balancing 'on-message' and 'off-message' content to render the former less overt or threatening and more palatable and approachable; (2) Obfuscating: using framing devices or genres that divert expectations or focus away from the game's persuasive intent; and (3) Distancing: employing techniques, such as the use of fictional or metaphorical representations of key issues or themes in order to increase the psychological gap between players' identities and beliefs and the game's characters and persuasive content. In the sections that follow, we expound 


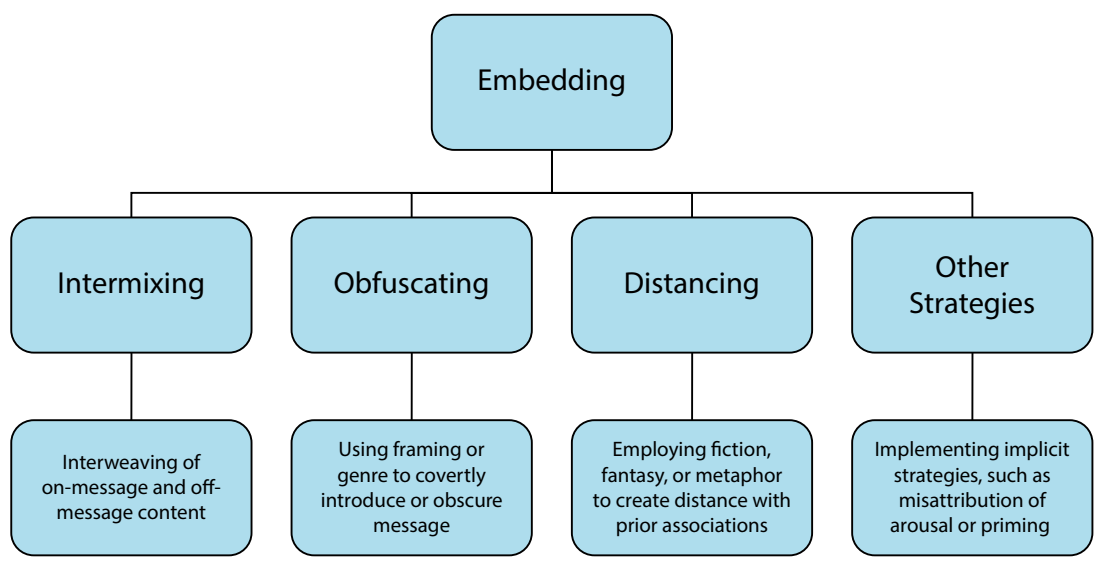

Figure 5.1: The 'embedded design' model.

upon these three strategies and provide concrete examples of our team's game designs to illustrate their implementation and cite the results of empirical investigations that support their efficacy. In addition, we will describe a set of additional embedding strategies that we have begun to explore in our work.

\section{Embedding through 'intermixing'}

One means of embedding persuasive content within a game's design is a strategy we have come to refer to as 'intermixing': balancing or interweaving on-topic content with playful but persuasively off-topic (or off-focus) content that either distracts from the intended message of the game or helps ease player into the game's message or aims. This strategy, when implemented effectively, reduces the likelihood of players experiencing the game as a top-down attitude or behavior change intervention and offsets the serious (or potentially uncomfortable) tone of the 'on-message' components of the game with content that has comparatively more levity or humor.

Our team has implemented and tested the intermixing strategy in several game designs. To cite one illustrative example, Awkward Moment (2012) is a party game for pre- and early-adolescent players that aims to reduce social biases, including gender stereotypes in science, technology, engineering, and math (STEM) domains (see Figure 2). In Awkward Moment, players begin with a hand of five 'reaction cards'; these cards describe potential responses to the game's 'awkward moments', including actions (e.g., 'Scream your head off', 'Write a blog post about it', 'Talk it out'), exclamations (e.g., 'Rats!', 'OMG', 'No way!'), and frames of mind (e.g., 'Get serious', 'Relax', 'Channel your inner warrior'). During each round, one player serves as the 


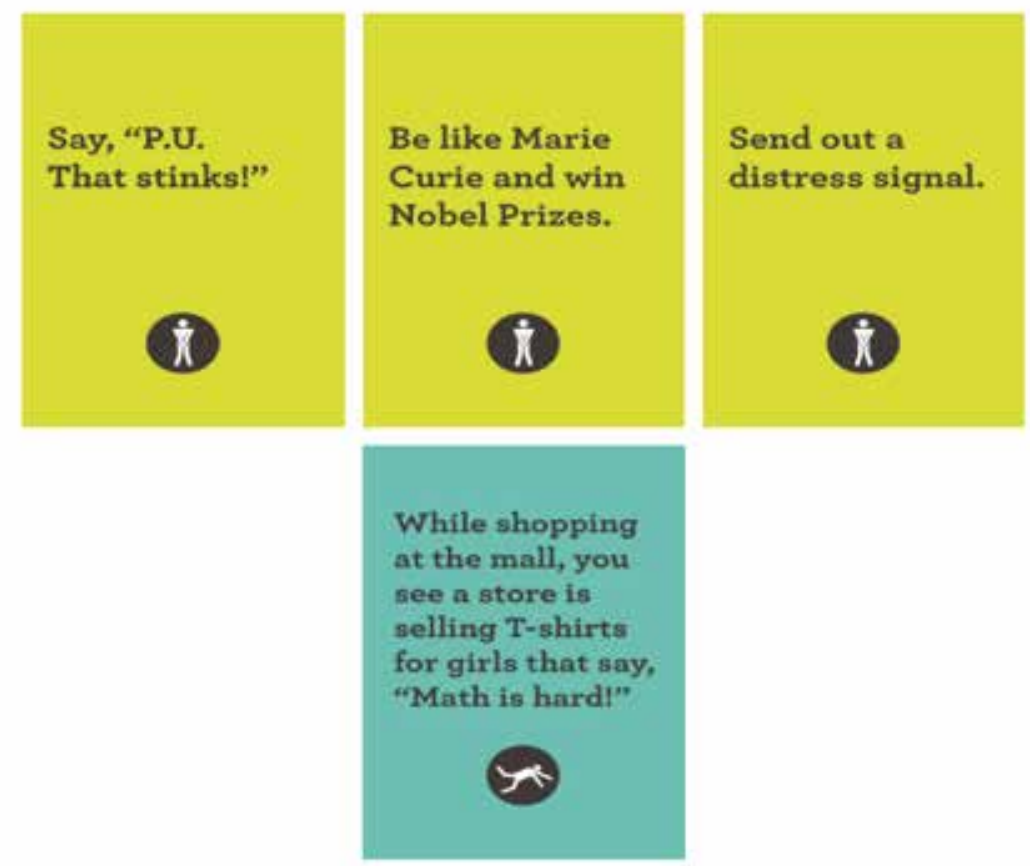

Figure 5.2: Sample Reaction Cards (green) and a sample Moment Card (blue) from Awkward Moment.

'Decider' and draws a 'Moment Card' that poses a hypothetical situation (e.g., 'Somebody hacks your Facebook account and changes your status to "Girls are stupid".'), to which the other players respond by submitting a Reaction Card face-down. The Decider then reads each of the submitted cards and selects a winner for the round. The game aims to stimulate thought and discussion about responses to social and academic dilemmas, particularly situations that involve bias against girls and women in STEM. A subset of the cards in the Moment deck present situations in which a female is a target of stereotypes. In some situations, players imagine being a target themselves. The game's deck of Moment Cards contain examples depicting on-topic scenarios related to gender bias in STEM (see Figure 5.2 for an example) as well as off-topic scenarios presenting awkward situations that do not directly pertain to social biases (e.g., 'You sit on ketchup' or 'There's a secret "Ugly Poll" at school, and you find out you were Number 3 on the list').

A key question that guided the iterative design of Awkward Moment was the ideal ratio of on-topic to off-topic Moments in the game. In line with the intermixing strategy, our empirical research showed that presenting a lower ratio of bias-themed to non-bias-themed Moments proved much 
more effective at shifting players' attitudes and perceptions. One of our controlled experiments (Kaufman \& Flanagan, 2015) revealed that youth participants randomly assigned to play an 'intermixed' version of the game (with approximately $45 \%$ of the Moment Cards depicting gender bias in STEM) exhibited statistically significant higher post-game levels of perspective-taking compared to participants assigned to play an 'overloaded' version of the game (with $75 \%$ of the Moment Cards pertaining to bias). In another experiment, an 'intermixed' version of the game produced a threefold increase in players' likelihood of associating women and science after one gameplay session. In both of these studies, we observed little evidence of players noticing, let alone reacting against, the game's persuasive content because it was not the ostensible subject or focus of the game.

An additional study involving a new version of the game for adults (depicting workplace scenarios) and utilizing the same methodology as the aforementioned experiment revealed the same pattern of results with adult participants. Those participants assigned to play an 'overloaded' version of the game exhibited significantly greater negative affect (including the distinct response of feeling 'fed up' by the end of the game) and a lower level of concern about the issue of social biases compared to participants assigned to either an 'intermixed' game condition or a no-game control condition (Kaufman \& Flanagan, 2018A). In sum, these findings confirm that overrepresenting serious, persuasive content within the game triggered players' reactance - and that this defensiveness prevented them from shifting their mindsets and perceptions after play.

In recent work, we have explored how the intermixing strategy might also be effectively implemented in a game's presentation of diverse characters as a means of reducing gender bias in STEM. In the time travel-themed strategy game The Luminists, players compete to 'restore' the most scientific and technological discoveries that have been undone by the unraveling of time by 'recruiting' real-life STEM role models whose skills and expertise assist them in their quest. In line with prior work demonstrating the beneficial impact of exposure to counter-stereotypical role models for lowering social biases and increasing STEM aspirations and pursuits (e.g., Dasgupta \& Asgari, 2004), the primary underlying goal of the game was to present a host of positive female STEM role models to young female players. At the same time, we predicted that 'intermixing' female and male STEM role model 'luminists' (rather than presenting a higher ratio of female to male luminists) would enhance the efficacy of the game-both by making the intended goals of the game less overt and by reinforcing equity rather than imbalanced participation in STEM between the genders. An initial experimental study involving a 
sample of female youth participants supported this prediction (Kaufman \& Flanagan, 2018B). In this study, we compared two versions of the game that differed in their ratio of male-to-female scientists in the set of eight presented to players - one in which there were equal numbers of male and female scientists and one in which six of the eight scientists were female. The results revealed that, compared to participants in a no-game control condition, participants assigned to play the 'intermixed' version of the game (but not those assigned to the 'imbalanced' version) exhibited significantly higher levels of psychological connection to the game's luminists and, as a result, greater aspirations to pursue computer programming and other STEM careers and higher self-efficacy in STEM.

Intermixing is counterintuitive. On the surface, the strategy may seem as though it would be less effective. Yet despite the fact that players are exposed to less focal content (e.g., fewer scenarios depicting occurrences of bias in Awkward Moment or fewer female role models in The Luminists), they are significantly more likely to accept and internalize (rather than reject and defend against) the game's underlying persuasive aims and messages. Our work to date has shown that the 'intermixing' strategy of balancing or interweaving on-topic, focal aspects of a game with off-message or off-topic content plays a central role in determining the efficacy of our persuasive games.

\section{Embedding through 'obfuscating'}

The second broad Embedded Design strategy that we have employed with great success is 'obfuscating': concealing or obscuring the true persuasive intent of a game by employing devices that divert players' attention and/ or allow for the covert introduction of persuasive themes or elements. One primary example of the obfuscating method is the decision to employ a game genre whose associated goals or expectations do not include the aim to change players' attitudes, beliefs, or behaviors. Indeed, our choice to design the aforementioned Awkward Moment as a fast-paced, social party game was a wholly intentional one. Triggering (and fulfilling) the anticipation of a fun, interactive play experience with an abundance of levity and laughter (achieved in part through the game's 'intermixing' of both serious and silly or absurd Moments and Reactions) created a 'safe' space for players to encounter and react to the game's heavier, on-message content with greater comfort-and greater candor. Our team's extensive playtesting and iteration of the game provided consistent support; through both unobtrusive observations of play sessions and post-game interviews 
with youth testers, playgroups generally approached the game with a strong and sustained spirit of levity and amusement, yet rarely showed signs of subverting the game's more serious moments (or Moments), even among older, more experienced (or even more 'jaded') players at venues such as the PAX or GenCon gaming conventions. Moreover, even when asked directly what they believed the true goals of the game were, players rarely identified the game's primary aim of challenging gender stereotypes in STEM domains but rather focused more broadly on the game's general focus on reacting to a variety of social situations (further evidence of the successful implementation of the 'intermixing' strategy) as well as a number of genreconsistent goals, such as the enjoyment of the game's social dynamics and the amusingly random or serendipitous pairings of Moment and Reaction cards that emerged.

In developing a second game with the same primary aim as Awkward Moment - to combat stereotypes and reduce prejudices-we went even a step further in using the party game genre to obfuscate the underlying goals. Buffalo: The Name Dropping Game (2012) is ostensibly a rapid-fire group trivia game: players flip a card from each of the game's two decks (one containing adjectives and the other nouns) and race to be the first to shout out the name of a real or fictional person who matches the revealed pair of descriptors (see Figure 5.3). What most players do not realize (and, as playtests and interviews have revealed, are quite surprised to learn) is that the game's deceptively simple design was based on an established psychological premise: exposure to a plethora of counter-stereotypical or otherwise unexpected exemplars (to which players are necessarily exposed given the game's random pairings of attributes and social categories) reliably reduces individuals' levels of stereotyping and prejudice. In a given play session, for example, players may be invited to name such diverse exemplars as a 'charismatic techie', 'rugged fashion designer', 'tattooed visionary', and 'Iranian poet'. Indeed, our own controlled experiments investigating the impact of Buffalo (Kaufman \& Flanagan, 2015) revealed that players of a single session of the game, compared to participants in a no-game control condition, exhibited significantly higher levels of social identity complexity (i.e., greater diversity and inclusiveness in their perception of their primary identity groups, which is a predictor of tolerance and egalitarianism; see Roccas \& Brewer, 2002) and universal orientation (i.e., a measure of global non-prejudice; see Phillips \& Ziller, 1997). Thus, despite—or, we would argue, because of - players' general failure to realize or recognize the game's persuasive goals and mechanism, the game successfully shifts players' conceptions of their own and others' identities simply by virtue of playing 

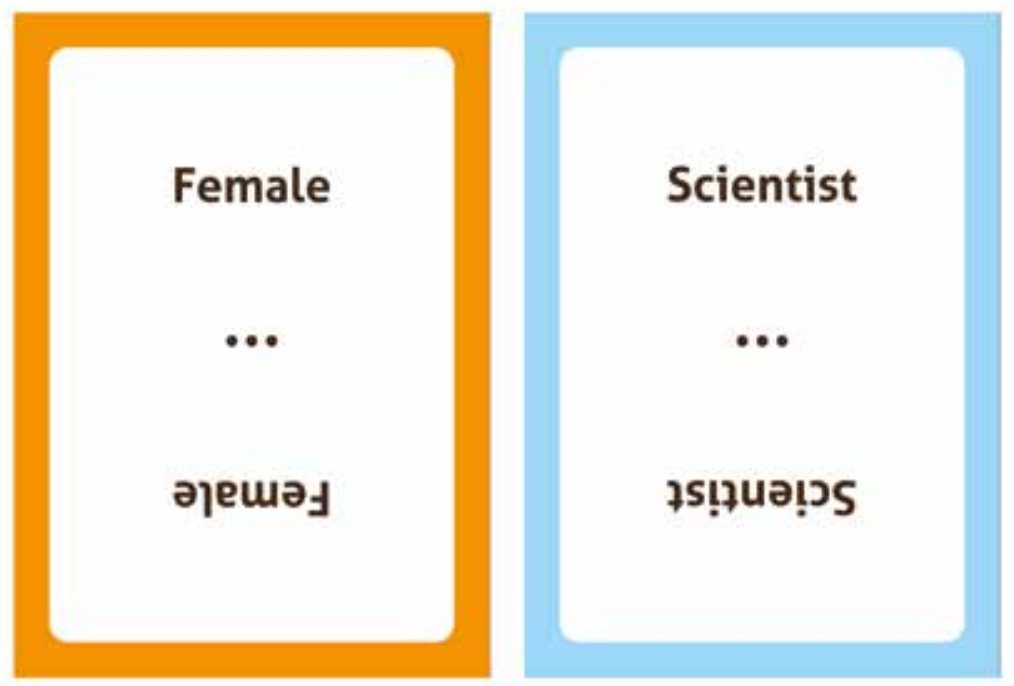

Figure 5.3: Sample card pairing from Buffalo: The Name Dropping Game.

the game and both offering and being exposed to a plethora of exemplars of cross-cutting identity groups and associated traits. Moreover, even in cases when players recognize how their own biases might have influenced their performance in the game (e.g., one playtest participant regretfully reflected on his and his group's failure to name a 'Hispanic lawyer' despite the fact that Sonia Sotomayor had recently been appointed to the Supreme Court), they by and large do not realize that this was, in fact, a focal outcome intended by the game's designers.

With both Awkward Moment and Buffalo, we employed yet another means of obfuscation, one that is particularly rare among persuasive games: we deliberately avoided disclosing the aims of the game in the descriptions provided to players on the game box and in the instructional materials and instead used deliberately neutral language to present and explain the game. This choice of neutral language represents a second obfuscation strategy: the use of framing devices that emphasize features of the game other than its focal subject matter or persuasive aims. Indeed, we predicted that simply revealing before play that either game dealt in some way with social biases and stereotypes could dramatically reduce players' enjoyment of the game or the game's impact, in part because such 'forewarning' would likely raise either conscious or unconscious defenses in players to resist the game's perceived intent. An initial pair of randomized experiments (Kaufman \& Flanagan, 2015) suggested that this was indeed the case. Holding 
all other game elements constant, adolescent players of Awkward Moment who were randomly assigned to a 'stereotype frame' condition (and were informed prior to play that the game dealt with 'awkward social stereotypes'), compared to those assigned to a 'situation frame' condition (who were told the game dealt with 'awkward social situations') reported finding the game significantly less fun and immersive and exhibited significantly less movement in their rejection of gender stereotypes. Likewise, playgroups who were told that Buffalo explored 'pop culture stereotypes' (compared to 'pop culture knowledge') did not show a reduction in their levels of prejudice, as assessed by the measure of universal orientation described above. These findings illustrate the basic premise of the Embedded Design model: persuasive games that overtly telegraph their intended purpose of shifting attitudes and mindsets are likely triggering mindsets in players that hinder the game's enjoyability and blunt its potential positive impact.

In addition to the selection of genres and the employment of framing language that diverts attention away from a game's true 'message', one final obfuscation strategy that we have applied is the delayed revelation of potentially threatening, counter-attitudinal, or alienating features or elements. Specifically, we have explored this technique to encourage greater psychological connection and higher levels of experience-taking with characters (Kaufman \& Libby, 2012): that is, greater immersion into the role and persona of protagonists in narrative and game worlds, particularly ones who belong to social 'outgroups'. This technique has previously proven effective for written narratives: for example, revealing a character's racial or sexual orientation -i.e., outgroup membership-later in a short story (once a psychological connection between reader and character had begun to take root) not only facilitated higher levels of experience-taking but also reduced prejudice levels toward the represented outgroups (Kaufman \& Libby, 2012). That is, initially obfuscating the potentially distancing (or stigmatizing) group membership of the character ultimately increased readers' receptiveness of the character's identity—and profoundly enhanced the persuasive impact of the story.

More recently, our team successfully applied this technique to encourage higher levels of experience-taking among male youth in our strategy board game Monarch, which puts players in the role of sibling princesses competing for the throne. Given the persistence of social norms that discourage 'genderswapping' play, particularly among boys (e.g., Martin, 1990; McCreary, 1994), we anticipated that revealing their character's gender prior to play would reduce experience-taking among male adolescent players. Conversely, in line with prior research, withholding this revelation for several rounds 
(during which players became acquainted with their characters and were gradually exposed to subtle clues about their true identity, including the use of gowns and pageantry as political instruments in the game) should more effectively ease players into their cross-gender roles. This was indeed the case: a controlled experiment (Kaufman \& Flanagan, 2018C) revealed that a sample of male youth randomly assigned to play the 'delayed revelation' version of the game reported higher levels of experience-taking with their princess characters compared to those assigned to an 'immediate revelation' version and, moreover, exhibited greater rejection of stereotypical gender norms (e.g., rejection of the association between 'female' and 'emotional' or 'weak') following gameplay.

\section{Embedding through 'distancing'}

The final Embedded Design strategy that we have explored in our work is the use of 'psychological distance' (Trope \& Liberman, 2010) to create a safe space between individuals and the serious or sensitive themes or topics explored or modeled by a game. By separating players from their real-life identities and prior knowledge, beliefs, and experiences, persuasive games can effectively circumvent players' reticence or reluctance and enhance the game's transformative potential—particularly in subject matter domains that may be uncomfortable or counter-attitudinal. By its very nature, an absorbing, immersive game should trigger a high level of psychological transportation (Green \& Brock, 200o), thereby distancing players from their real-life surroundings and true identities, which prior work has shown sets the stage for higher levels of enjoyment (Green et al. 2004) and persuasion (Green \& Brock, 2000; Green, Brock, \& Kaufman, 2004; Kaufman \& Libby, 2012). Indeed, we would argue that persuasive games that take too overt or literal an approach in their handling of controversial or sensitive topics have inherently less capacity to transport their players (and to provide an enjoyable experience) because they create too little psychological distance to explore those topics in a non-threatening fashion.

Beyond the psychological distance afforded by a highly transporting game, however, there are a number of specific distancing strategies that designers can use to increase the gap between players' real-life experiences and the ideas, encounters, and interactions that await them in the game. These strategies are derived from a number of distinct manifestations of psychological distance revealed by prior work (Trope \& Liberman, 200o). Perhaps the most elementary forms of distance is hypotheticality: rather than presenting situations that are drawn directly from players' real-life 
experiences (or situations that attempt to replicate or mirror those experiences), encouraging players instead to engage in 'what if?' scenarios provides a safe 'buffer' to explore even the most sensitive topics. Indeed, the value of hypotheticality was a key decision point in the design of Awkward Moment: each of the game's Moment Cards present a purely hypothetical situation and invites players to consider a host of alternative ways of responding. Rather than placing the players and their embarrassing moments or experiences with bias in the spotlight, the game allows players to envision and select responses for the unidentified second-person 'you' described in each of the game's Moments.

A second distancing mechanism that we utilize in our work is the fictionalizing of real-life issues and events - that is, the embedding of those elements within symbolic, fantastical, or metaphorical representations. This technique is by no means a new one: the use of fiction to disguise the focus or target of a story may be as old as written language itself. What is distinctive in our approach to using fictional representations in games is our endeavor to systematically compare different levels of explicitness (versus 'embeddedness') in those representations. To cite one example of this approach, our team has designed and studied two versions of the public health board game POX: Save the People (2011) intended to promote positive attitudes and valuations toward vaccination: one version $(P O X)$ that presented a relatively straightforward, realistic narrative about disease spread, and one version (ZOMBIEPOX) utilizing a more fantastical narrative about the spread of a 'zombie plague' (see Figure 5.4). Both games share the same essential rules and mechanics concerning the spread of infectious disease and the modeling of vaccination as an effective strategy for curtailing that spread, but they differ in the level of distance afforded by their representation of disease, infection, and death (or 'un-death' in the case of ZOMBIEPOX). A pair of controlled experiments comparing the impact of these two games on both adult and youth players revealed parity between the games (compared to a no-game control condition) in terms of their impact on players' valuation of vaccination as a public health solution. At the same time, however, players of the zombie-themed version of the game reported higher levels of psychological transportation and higher levels of empathic concern toward individuals with infectious diseases, as assessed by self-report measures (Kaufman \& Flanagan, 2016). Thus, the use of a more distanced, metaphorical representation of disease was not only effective at shifting attitudes toward a real-life health policy issue but, indeed, even more effective than the less distanced, realistic narrative at forging a bond of compassion between players and the real-life individuals symbolized by 

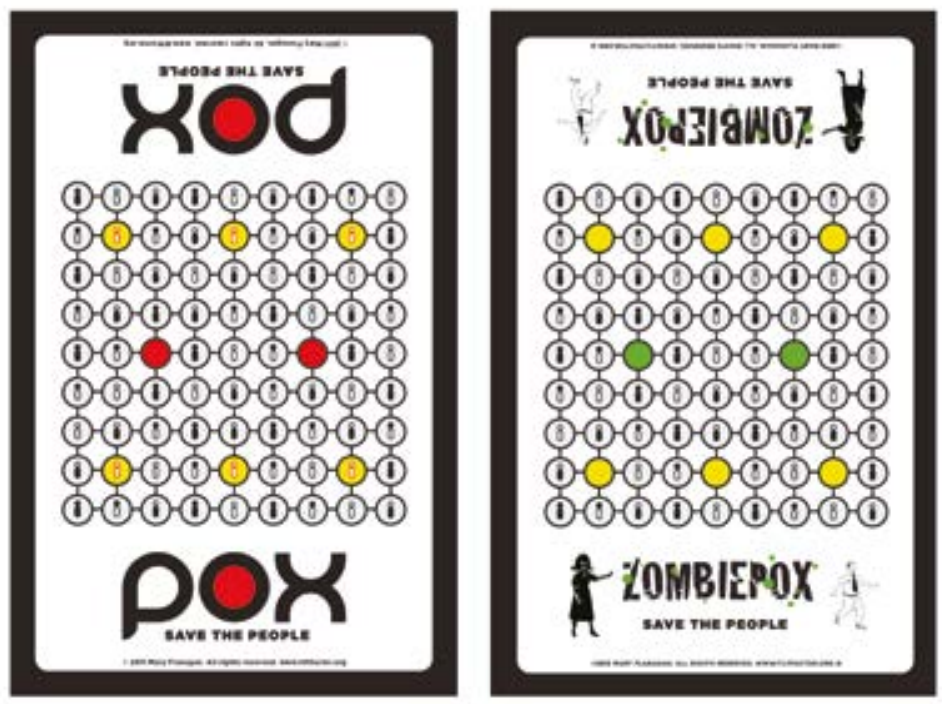

Figure 5.4: POX and ZOMBIEPOX game boards.

the zombies in the game. This finding lends further credence to our view that persuasive games utilizing elements of the Embedded Design model (such as distancing) are likely to be experienced as more transporting by players - and, consequently, more impactful at changing hearts and minds.

In our current work, we are exploring the benefits of fictionalized distancing for individuals creating narrativized accounts of their own real-life experiences - specifically, personal reflections on their experiences being the target of others' stereotypes and biased expectations, judgments, or behaviors. This project will explore the therapeutic and cathartic value of creating interactive 'text adventures' that are based on stressful or traumatic real-life occurrences but provide creators with the safety (and creative license) afforded by the fictionalized re-telling and recounting of those life events. In this stream of research, we will investigate the effects of writing interactive narratives that are more fictionalized versus more strictly autobiographical, as well as the impact of a number of other distancing mechanisms - such as the narrative voice (e.g., a more distanced thirdperson voice versus the less distanced first and second-person perspectives) employed by authors - on the emotional benefits of narrativizing one's own lived experiences. This work has begun to show the benefit of distancing for particular populations, including individuals with lower levels of self-esteem or higher levels of chronic self-focus (Green et al., 2017). 


\section{Additional embedding strategies}

In addition to the strategies described above, we have begun to explore and identify several other embedding techniques in our work. First, we have shown that employing the psychological phenomenon of misattribution of arousal - by which heightened arousal can be incorrectly caused by a situational cue or stimulus put into place in the environment (Schacter \& Singer, 1962) — can be a means of redirecting explanations for aversive arousal triggered by persuasive content to different sources, thus changing the emotional experience associated with that arousal. To illustrate, Geoff Kaufman (one of the authors of this chapter) and collaborators have used this technique in a board game called Outbreak, which aims to increase scientific curiosity among youth populations who are underrepresented in science fields by employing a suspenseful horror theme as a means of nudging players to perceive that any anxiety caused by the science content was, instead, triggered by the game's theming and narrative (To et al., 2018).

In addition, we have begun to explore the design landscape for implicit methods of persuasion: techniques that trigger mindsets, emotions, and goals in covert ways or in the absence of users' conscious deliberation or intention (Gawrwonski \& Bodenhausen, 2006). Foremost among these techniques is priming the situational activation of mental constructs by stimuli that are strategically placed in the current environment. Priming is one of the most ubiquitous concepts in the psychological literature and, indeed, has already begun to be employed in gaming contexts. Riot Games received notable press coverage of their implementation of priming within their popular title League ofLegends: specifically, a set of informal studies showed that altering the font color of game tips presented on loading screens (e.g., using blue text instead of white in a message about cooperation) reduced subsequent rates of hostility among players. In our work, we are employing similar methods in designing interface elements (such as CAPTCHAs) that include images or text to prime positive affective states prior to interactions in online spaces, such as gaming environments and discussion boards (Seering et al., 2019).

\section{Conclusion}

The Embedded Design model offers a number of easily implementable, evidence-based techniques that promise to revolutionize the ways that game developers tackle serious content issues and make more effective and more 
enjoyable games. As illustrated by examples from our own game designs and accompanying empirical work, the more covert, 'stealthy' approaches derived from the Embedded Design model result in persuasive games that are remarkably more transporting and impactful compared to games in which the message or material is presented more overtly or directly (see Kaufman \& Flanagan, 2015). Additionally, the model advances the conversation around the application of psychological principles in games and builds on other theoretical and practical formulations for understanding games, such as models of game design patterns (Bjork \& Holopainen, 2004).

It is important to note that the strategies described here are by no means intended to be comprehensive. Our team has just begun to discover the potential of such techniques. Each new game project we (and others) take on sets the stage for new manifestations and applications of Embedded Design to emerge and, as a result, extend, enhance, and refine the design model introduced here. Moreover, although the game case studies we presented here to exemplify the model were non-digital, the principles and practices suggested by the model are intended to be broadly implemented across all game platforms and media delivery formats. Indeed, the greater flexibility and control afforded by the creation of digital games (e.g., in their revelation of information or representation of characters) open up a world of new possibilities for embedding that designers can consider, implement, and test in their own work (e.g., Christiansen, 2014).

\section{Acknowledgments}

This chapter is an extended version of a paper that originally appeared in the Transactions of DiGRA (ToDiGRA) in 2016. We are extremely grateful to the Digital Games Research Association for granting the permission to present this modified version here.

\section{References}

Bjork, S., \& Holopainen, J. (2004). Patterns in Game Design. Newton Center, MA: Charles River Media.

Bogost, I. (2007). Persuasive Games: The Expressive Power of Videogames. Cambridge, MA: MIT Press.

Brehm, J.W. (1966). A Theory of Psychological Reactance. New York, NY: Academic Press. 
Centieiro, P., Romão, T., \& Dias, A.E. (2011). A Location-Based Multiplayer Mobile Game to Encourage Pro-Environmental Behaviors. In Proceedings of the 8th International Conference on Advances in Computer Entertainment Technology (Lisbon, Portugal, November 2011). New York, NY: ACM Press.

Christiansen, P. (2014). Presence and Heuristic Cues: Cognitive Approaches to Persuasion in Games. In Proceedings of the Digital Games Research Association Conference (Salt Lake City, UT, August 2014). Retrieved 16 May 2018 from http://www.digra.org/digital-library/publications/ presence-and-heuristic-cues-cognitive-approaches-to-persuasion-in-games/.

Dasgupta, N., \& Asgari, S. (2004). Seeing Is Believing: Exposure to Counterstereotypic Women Leaders and Its Effect on the Malleability of Automatic Gender Stereotyping. Journal of Experimental Social Psychology, 4o, 642-658. https:// doi.org/10.1016/j.jesp.2004.02.003.

De la Hera Conde-Pumpido, T. (2013). A Conceptual Model for The Study of Persuasive Games. Proceedings of the Digital Games Research Association Conference (Atlanta, GA, August 2013). Retrieved 3oJune 2017 from http://www.digra.org/digital-library/ publications/a-conceptual-model-for-the-study-of-persuasive-games/.

Dunbar, N.E., Wilson, S.N., Adame, B.J., Elizondo, J., Jensen, M.L., Miller, C.H., ... \& Burgoon, J.K. (2013). MACBETH: Development of a Training Game for the Mitigation of Cognitive Bias. International Journal of Game-Based Learning, 3 , 7-26. https://doi.org/10.4018/ijgbl.2013100102.

Flanagan, M. (2009). Critical play: Radical game design. Cambridge, MA: MIT Press. . \& Nissenbaum, H. (2014). Values at Play in Digital Games. Cambridge, MA: MIT Press.

Fiellin, L.E., Hieftje, K., Duncan, L.R., Sawyer, B.G., \& Fiellin, D.A. (2014). A Videogame for Substance Use Prevention and HIV Risk Reduction in Young Adolescents. Drug and Alcohol Dependence, 140, e58-e59. https://doi.org/10.1177/1740774516637871.

Gawronski, B., \& Bodenhausen, G.V. (2006). Associative and Propositional Processes in Evaluation: An Integrative Review of Implicit and Explicit Attitude Change. Psychological Bulletin, 132, 692-731. https://doi.org/10.1037/0033-2909.132.5.692.

Green, M.C., \& Brock, T.C. (200o). The Role of Transportation in the Persuasiveness of Public Narratives. Journal of Personality and Social Psychology, 79, 701-721. https://doi.org/10.1037/0022-3514·79·5·701.

_., Brock, T.C., \& Kaufman, G.F. (2004). Understanding Media Enjoyment: The Role of Transportation into Narrative Worlds. Communication Theory, 14, 311-327. https://doi.org/10.1111/j.1468-2885.2004.tboo317.x.

__., Kaufman, G., Flanagan, M., \& Fitzgerald, K. (2017). Self-Esteem and Public Self-Consciousness Moderate the Emotional Impact of Expressive Writing about Experiences with Bias. Personality and Individual Differences, 116, 212-215. https:// doi.org/10.1016/j.paid.2017.04.057 
Huizinga, J. (1938). Homo Ludens. Versuch Einer Bestimmung des Spielelements in der Kultur. Hamburg: Rowohlt.

Kaufman, G., \& Flanagan, M. (2015). A Psychologically 'Embedded' Approach to Designing Games for Prosocial Causes. Cyberpsychology:Journal of Psychosocial Research on Cyberspace, 9(3). https://doi.org/10.5817/CP2015-3-5.

-. (2018A). How Awkward! Shifting Workplace Biases with a Social Game. Manuscript under review.

- (2018B). How STEM Role Models Shift Thinking About STEM Careers in a Time Travel Game. Manuscript under review.

- (2018C). Not Just for Girls: A Strategy Princess Board Game that Challenges Gender Stereotypes. Manuscript under review.

- (2016). Lost in Translation: Comparing the Impact of an Analog and Digital Version of a Public Health Game on Players' Perceptions, Attitudes, and Cognitions. International Journal of Gaming and Computer-Mediated Simulations, 5, 1-9. 10.4018/jgcms.2013070101.

Kaufman, G.F., \& Libby, L.K. (2012). Changing Beliefs and Behavior Through Experience-Taking.Journal of Personality and Social Psychology, 103, 1-16. https:// doi.org/10.1037/aoo27525.

Martin, C.L. (1990). Attitudes and Expectations About Children with Nontraditional and Traditional Gender Roles. Sex Roles, 22, 151-166. https://doi.org/10.1007/ BFo0288188.

McCreary, D.R. (1994). The Male Role and Avoiding Femininity. Sex Roles, 31, 517-531. https://doi.org/10.1007/BFo1544277.

Phillips, S.T., \& Ziller, R.C. (1997). Toward a Theory and Measure of the Nature of Nonprejudice.Journal of Personality and Social Psychology, 72, 420-434. https:// doi.org/10.1037/0022-3514.72.2.420.

Pronin, E., Lin, D.Y., \& Ross, L. (2002). The Bias Blind Spot: Perceptions of Bias in Self Versus Others. Personality and Social Psychology Bulletin, 28, 369-381. https:// doi.org/10.1177/0146167202286008.

Roccas, S., \& Brewer, M.B. (2002). Social Identity Complexity. Personality and Social Psychology Review, 6, 88-106. https://doi.org/10.1207/S15327957PSPRo602_01.

Schachter, S., \& Singer, J. (1962). Cognitive, Social, and Physiological Determinants of Emotional State. Psychological Review, 69, 379-399. https://doi.org/10.1037/ hoo46234.

Seering, J., Fang, T., Damasco, L., Chen, M., Sun, L., \& Kaufman, G. (2019). Designing User Interface Elements to Improve the Quality and Civility of Discourse in Online Commenting Behaviors. In Proceedings of the of the 2019 CHI Conference on Human Factors in Computing Systems (Glasgow, UK, May 2019). Retrieved 11 June 2017 from https://www.andrew.cmu.edu/user/jseering/papers/Seering\%20 et\%20al\%202019\%2oPersuasive\%2oCAPTCHAs.pdf. 
To, A., Holmes, J., Fath, E., Zhang, E., Kaufman, G., \& Hammer, J. (2018). Modeling and Designing for Key Elements of Curiosity: Risking Failure, Valuing Questions. Transactions of the Digital Games Research Association, 4. Retrieved 23 May 2017 from http://www.digra.org/digital-library/publications/modeling-and-designingfor-key-elements-of-curiosity-risking-failure-valuing-questions/.

Trope, Y., \& Liberman, N. (2010). Construal-Level Theory of Psychological Distance. Psychological Review, 117, 440-463. 10.1037/a0018963.

Van't Riet, J., Meeuwes, A.C., Van der Voorden, L., \& Jansz, J. (2018). Investigating the Effects of a Persuasive Digital Game on Immersion, Identification, and Willingness to Help. Basic and Applied Social Psychology, 1-15. https://doi.org/1 0.1080/01973533.2018.1459301.

Worchel, S., \& Brehm, J.W. (1970). Effect of Threats to Attitudinal Freedom as a Function of Agreement with the Communicator. Journal of Personality and Social Psychology, 14, 18-22. https://doi.org/10.1037/hoo28620.

\section{About the authors}

Geoff Kaufman is the founder of the eHeart Lab and an Assistant Professor in the Human-Computer Interaction Institute (HCII) at Carnegie Mellon University. His primary research focuses on how experience-taking - the mental simulation of characters' experiences in fictional narratives, virtual worlds, or games - can change individuals' self-concepts, attitudes, behaviors, and emotions.

Mary Flanagan is an inventor, artist, writer, and designer whose design work, installations, and writing forge a unique vision of technology, pop culture, and creativity. She is the author of Critical Play (2009) and Values at Play in Digital Games (2014), both published by the MIT Press.

Max Seidman is a graduate of Dartmouth College with a B.A. in engineering sciences with a focus on human-centered design. When he is not gaming, he spends his time working on games, designing games, and thinking critically about games (with the occasional break to eat and sleep). 


\section{Part II}

Designing Persuasive Games 



\title{
Introduction to Part II
}

\author{
Ben Schouten \& Martijn Kors
}

This part of the book sheds light on the design values that could incite player experiences stemming from players' needs, emotions, or identity concerns and how to translate these into design strategies offering novel opportunities for persuasion. The focus is on current practices where games and playful interactions are embedded in trans-medial applications. We see a trend in which games are more open social systems in which players cooperate, plan, organize, and create in different contexts and environments (education, training, lifestyle). Several mock-ups and prototypes that target specific user groups are presented in this section, resulting in a set of exemplary designs and design guidelines.

In the first chapter of this second part, Martijn Kors and his colleagues share the lessons learned during the design process of $A$ Breathtaking Journey, a mix-reality game that puts players in the shoes of refugees during their flee journey. This study explores from a qualitative perspective the potential of this VR experience to arouse empathy among players. To do so, this chapter establishes theoretical links between two fields, namely one exploring games designed to foster empathic engagement to persuade and the other studying human-computer interaction artifacts designed to elicit empathy. The results of this study delve into three design opportunities in VR environments to foster empathic interactions: visceral engagement, reflective moments, and affective appeals.

In Chapter 7, Lindsay Grace reflects on why games are the right or potentially wrong medium for delivering persuasive messages. In 'Macro, Micro and Meta-Persuasive Play to Change Society', the author investigates why public discourse comes to need games as a vehicle for communication and argumentation. In his chapter, Grace claims that there are three broad categories of persuasive play that should be further explored from both an academic and an industrial perspective. These three categories are macropersuasion, micro-persuasion, and meta-persuasion. The goal of the chapter is to help others in the persuasive games space examine these practices to refine their own work, develop appropriate strategies for combating the unintended results of such work, and provide a topographic view of how such strategies might be applied elsewhere.

In Chapter 8, Menno Deen and Eline Muijres narrate the design process of VilDu?!, a game — or therapeutic tool—for sexually abused children 
that was designed following a research-through-design approach. The lessons learned from the design experience and the game's implementation process in therapy sessions have contributed to a design approach for therapeutic games. The authors focus on three affordances of games useful to facilitate therapeutic conversations about sexual abuse, namely focus, non-normativity, and ambiguity. The conclusions of this chapter also enter the discussion on how games can be used to enrich therapy practices and how they could be implemented.

In Chapter 9, Sun Joo Ahn studies how user experiences in virtual worlds shape and transform individual attitudes and behaviors in the physical world. Embodied experiences in virtual reality (VR) involve the reproduction of sufficiently realistic sensory information so that users are able to see, hear, and feel experiences as if they are going through them at the moment. The question is, however, how these virtual experiences carry over into the physical world to impact attitudes and behaviors beyond playing sessions. Underlying mechanisms of embodied experiences that produce these outcomes are discussed in the context of media affordances or interactions between novel attributes of VR and user perceptions of them. In her chapter, she discusses design solutions to maximize persuasive effects, illustrating this with case studies. She also elaborates on the limitations of embodied experiences and identifies the most appropriate tasks for applying embodied experiences in VR. 


\title{
6. A Breathtaking Journey. Appealing to Empathy in a Persuasive Mixed- Reality Game
}

\author{
Martijn Kors, Gabriele Ferri, Erik D. van der Spek, Cas Ketel, \& \\ Ben Schouten
}

\begin{abstract}
Persuasive games are designed for a variety of objectives, from marketing to healthcare and activism. Some of the more socially aware ones cast players as members of disenfranchised minorities, prompting them to see what they see. In parallel, designers have started to leverage system-immersion to enable players to temporarily feel like another person, to sense what they sense. From these converging perspectives, we hypothesize a stilluncharted space of opportunities at the crossroads of games, empathy, persuasion, and system-immersion. We explored this space by designing A Breathtaking Journey, a mixed-reality game providing a first-person perspective of a refugee's journey. A qualitative study was conducted to tease out empathy-arousing characteristics, provide insights on empathic experiences, and contribute three design opportunities: visceral engagement, reflective moments, and affective appeals.
\end{abstract}

Keywords: mixed-reality game; persuasive games; empathy; research-through-design

\section{Introduction}

Games are no longer designed for entertainment purposes alone and increasingly play a role in marketing, education, healthcare, and political activism. Some games simulate experiences; others aim at persuasion. Games with the latter objective are typically produced with the designer's intent to shape

Hera, T. Dela, J. Jansz, J. Raessens, B. Schouten, Persuasive Gaming in Context. Amsterdam: Amsterdam University Press, 2021 DOI 10.5117/9789463728805_CHo6 
how players think and feel about the represented issue in reality (Bogost, 2007; Kors, van der Spek, \& Schouten, 2015), with the more socially aware ones designed not for expressly commercial or promotional purposes (i.e., advergames [De la Hera, 2019]) but for addressing social and humanitarian issues. These games often have players temporarily take on the role of specific actors-for example the disadvantaged, the marginalized, or the dispossessed - to communicate how it feels to be in a certain condition. In parallel, several HCI (Human-Computer Interaction) solutions have been developed over the years exploring empathy as a desired outcome. In this chapter, we aim to connect the two fields, namely: (1) games that foster empathic engagement to persuade, and (2) HCI artifacts designed to elicit empathy as a main objective. In the following paragraphs, we further unpack these two perspectives on empathy and digital games/artifacts. The first one is slightly more functionalistic and foregrounds empathy as a desirable means to leverage in persuasion. The second brings aesthetic experiences to the forefront and explores artifacts aimed at altering users' perceptions, thus possibly simulating empathic states. We explored the connection of these two fields by designing A Breathtaking Journey, a mixed-reality game providing a first-person perspective of a refugee's journey.

\section{Empathy-oriented persuasive games}

The particular empathy-arousing capacities of games have been recognized in different studies (Belman \& Flanagan, 2010), for example as a result of simulated role-taking (Peng, Lee, \& Heeter, 2010) or players adopting goals of the protagonist (Lankoski, 2007).

Games that are subsumed under the umbrella of empathy games (Bartelson, 2013) may stimulate both the cognitive and affective states of empathy (Boltz, Henriksen, \& Mishra, 2015; Belman \& Flanagan, 2010)—empowering players to explore alternate points of view —and foster a sense of shared similarity and empathic concern for individuals and groups with whom they may not have direct contact. Belman and Flanagan (2010) point to PeaceMaker (ImpactGames, 2007) as an exemplary empathy game:

'Cognitive empathy is involved in gameplay [...]. To make progress [...] players have to consider the perspectives of a variety of stakeholders, rather than only that of their own side. [...] The game requires one to think carefully about the perspectives of a wide range of stakeholder groups [...] Policy decisions that agitate a stakeholder group too much can potentially derail the peace process.' (p. 12). 
With players, journalists, and designers often asserting an uncritical and almost charitable stance concerning the design of such games (e.g., Bartelson, 2013), it is often overlooked that many are also intentionally designed to shape attitudes towards represented issues (Bogost, 2007). As Boltz et al. (2015) argue, 'well-designed empathy games can also encourage [players] to evaluate choices and consequences, and to question the system a game represents.' (p. 8). Games such as PeaceMaker (ImpactGames, 2007) and This War of Mine (11 bit studios, 2014) do not only ask players to empathize with certain actors, they also present the player with arguments that might cause one to think and feel differently about the people, events, or situations that are represented in these games (Belman \& Flanagan, 2010; de Smale, Kors, \& Sandovar, 2017).

\section{Empathy-oriented use of system-immersion}

Empathy has also been conceptualized in the domain of Human Computer Interaction (HCI), in particular with respect to artifacts that-while not being full-fledged games - still leverage a degree of playfulness in engaging their users (Schouten, Deen, \& Bekker, 2010). Specifically, the interplay between system-immersion and phenomenology is opening new design spaces: the rapid and widespread diffusion of VR (virtual reality), AR (augmented reality), and wearables has made it feasible and relatively affordable to create a variety of artifacts that turn an experience into someone else's phenomenological perception. Design research has drawn upon pragmatist phenomenology to argue that interacting with technology in society 'requires us to understand the experiences of [a] person in relation to ourselves and it is [there] that we identify empathy' (Fiore, Wright, \& Edwards, 2005, p. 131). To illustrate this, we highlight two approaches of using system-immersion for empathy-oriented playful HCI: first, immersive journalism, and second, wearable devices that modify the perception of one's own body. As we present our selected examples, we categorize them as playful inasmuch as they are not games. But they are not functionalistic tools either: as Lucero, Karapanos, Arrasvuori, and Korhonen (2014) argue, 'playfulness is a mindset whereby people approach every day, even mundane, activities with an attitude similar to that of paidia' (p. 36). De la Peña et al. (2010) discuss the possibilities of immersive journalism, a form of first-person experience of a journalistic reportage, "[allowing] the participant [...] to actually enter a virtually recreated scenario representing the news story [...] [that] affords the participant unprecedented access to the sights and sounds, and possibly feelings and emotions' (De la Peña et al., 2010, p. 2). In a 
similar vein, Arora, Pousman, and Milk developed Clouds Over Sidra (Arora, Pousman, and Milk, 2015), a 36o-degree film in partnership with the United Nations, following Sidra, a twelve-year-old girl living in the Za'atari camp in Jordan. On the production company's website, co-director Arora describes: by leveraging breakthrough technologies, such as virtual reality, we can create solidarity with those who are normally excluded and overlooked, amplifying their voices and explaining their situations' (Arora et al., 2015). And indeed, filmmaker and digital artist Chris Milk has recently argued for VR constituting an 'ultimate empathy machine' (Milk, 2015, 3:01). When playing Clouds Over Sidra, 'you're sitting there in [Sidra's] room, watching her, you're not watching it through a television screen, you're not watching it through a window, you're sitting there with her [...] when you look down, you're sitting on the same ground that she's sitting on [...] and because of that, you feel her humanity in a deeper way: you empathize with her in a deeper way' (Milk, 2015, para.13).

In addition to using technology to alter the viewpoint of the viewer, technological devices can also be used to stimulate the other senses and thereby alter the viewer's bodily perception, with the objective of making the viewer feel what someone else might feel. Marshall et al. (2011) and Benford et al. (2012) have experimented with devices built around militarystyle gas masks. Marshall et al. report on a horror-themed maze visited by volunteers wearing sensor-equipped gas masks, who were remotely observed by other participants through their video- and audio feeds. Semi-structured interviews showed the remote participants experiencing heightened fear, arguably because of their empathic bond with the volunteers inside the horror labyrinth, which was based on seeing what they saw and hearing them breathe. In a later study (Benford et al., 2012), they present Breathless, a physical installation for three participants wearing breath sensors. Breathless prompts one participant to sit on a swing, another to control its rhythm, and the last one to observe them from afar. All participants were instructed to synchronize their breathing and their movements. The authors concluded that the shared perceptions created an empathic relationship between the participants.

\section{An opportunity space: games, immersion, empathy, and persuasion}

The cases discussed so far suggest that system-immersion may be an effective vehicle to incite and support empathy. As such, it is surprising that systemimmersion, particularly in combination with empathy-arousal as persuasive appeal, is still largely absent from the repertoire of persuasive games. On 
the one hand, we have seen a variety of persuasive games following in the footsteps of PeaceMaker (ImpactGames, 2007), fostering empathy by presenting carefully simulated sequences of strategic actions and reactions. At the same time, Marshall et al. (2011) and Benford et al. (2012) exemplify an approach to empathy that is more grounded in a playful use of systemimmersion. Building on these insights about empathy and persuasion, we launched an exploratory project using RtD (Research through Design; see Zimmerman, Forlizzi, \& Evenson, 2007) to examine this opportunity space, produce relevant concepts, and test them with an experimental game. The following section reports on this project.

\section{A Breathtaking Journey}

A Breathtaking Journey (ABTJ) is a mixed-reality game meant to arouse empathy for refugees. ABTJ was born out of a project with Amnesty International in the Netherlands in 2014 and 2015 in which we set the objective of exploring how interactive media could help motivate people to change or reinforce their attitudes towards human rights-related issues. The design of ABTJ draws inspiration from empathy-supporting 'games for change' such as Hush (Antonisse \& Johnson, 2013) as well as from unusual immersive experiences such as Taphobos (Brown, Gerling, Dickinson, \& Kirman, 2015). ABTJ places the player in the shoes of a refugee who is fleeing from a war-torn country, hiding in the back of a truck to reach a safe haven. The virtual experience of ABTJ, delivered through an Oculus Rift Development Kit 2 head-mounted display (HMD) and over-the-ear headphones, is augmented with a range of physical elements including a respiration mask (housing an anemometer [breathing] sensor and two ultrasonic scent diffusers), a tangible contraption mimicking the inside of a truck, an unbalance motor to simulate movement, and a controlled shutter to drop objects on the player during the game (see Figure 6.1 and $6.2)$.

The augmentation of the virtual experience with real physical elements such as acceleration, wind, temperature, scent, and touchable materials has shown to support a sense of presence (Dinh, Walker, Hodges, Song, \& Kobayashi, 1999) and may therefore strengthen the persuasive effects in similar ways to how narrative-immersion drives (narrative) persuasion (Carpenter \& Green, 2012). We used the Unreal Engine 4 to render the $3 \mathrm{D}$ environment; drive the gameplay and narration; and facilitate the communication between sensors, actuators, and the game system. As an 


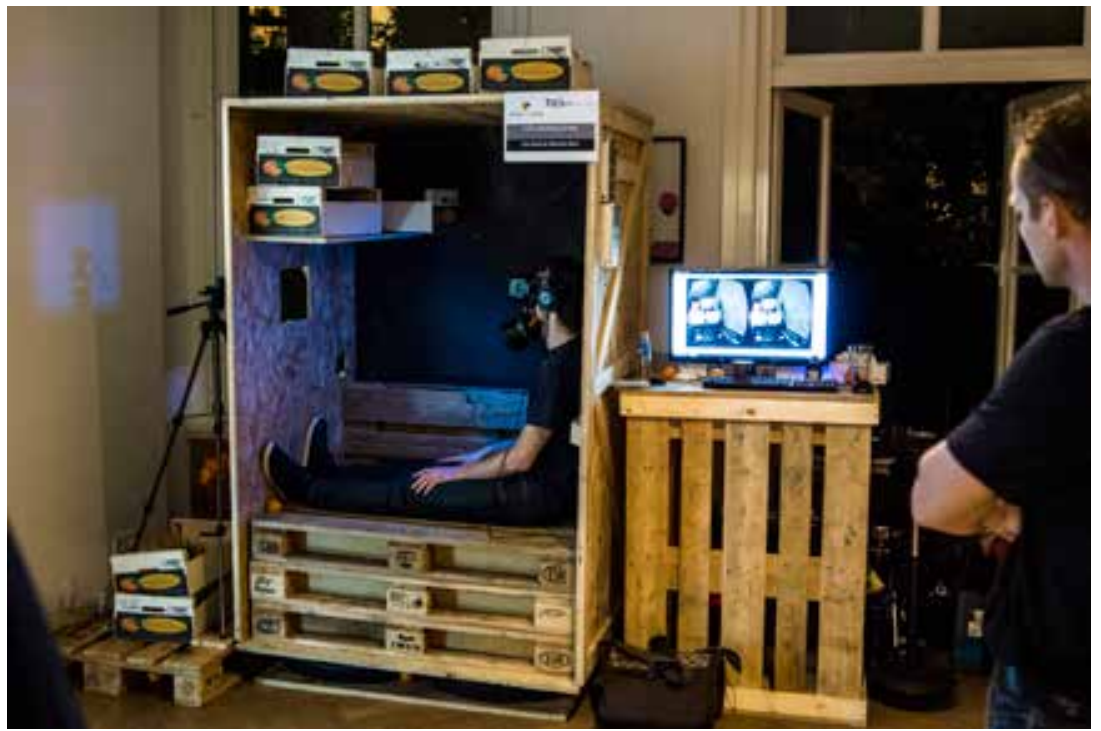

Figure 6.1

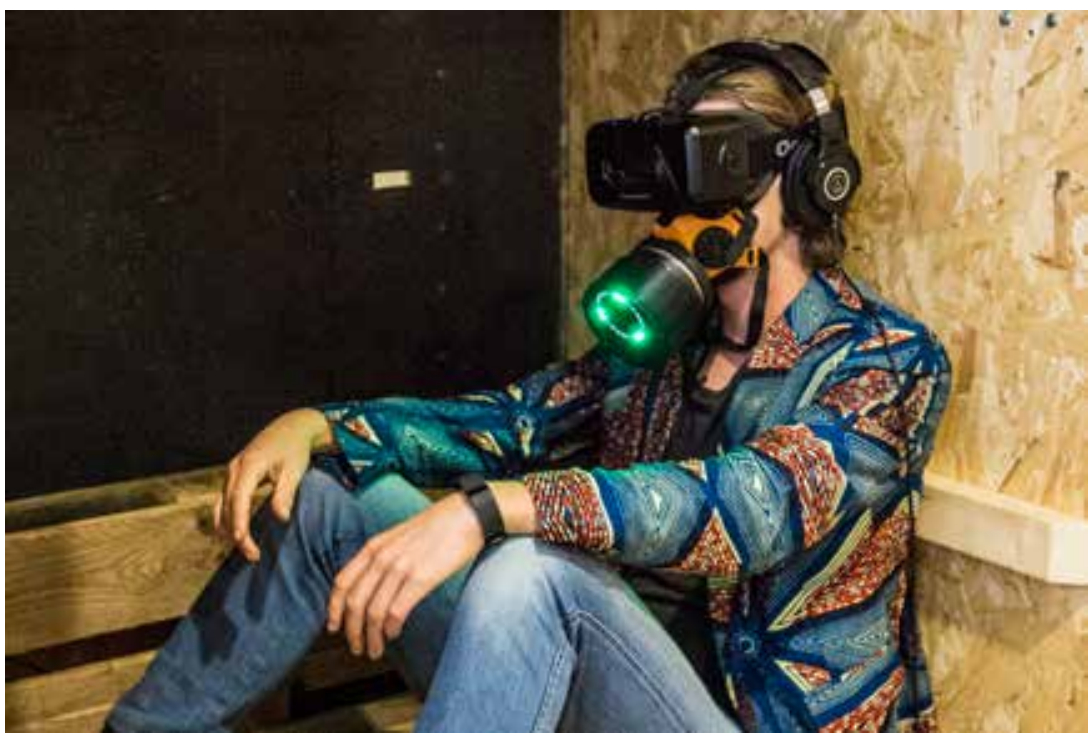

Figure 6.2

RtD project, ABTJ addresses the aforementioned gap between persuasive/ empathy games and 'empathy machines' by designing a game that: (1) generates a sense of presence through embodied and multi-sensory elements, (2) suggests complex emotions and visceral reactions, and (3) stimulates users to interpret ABTJ's narrative. 


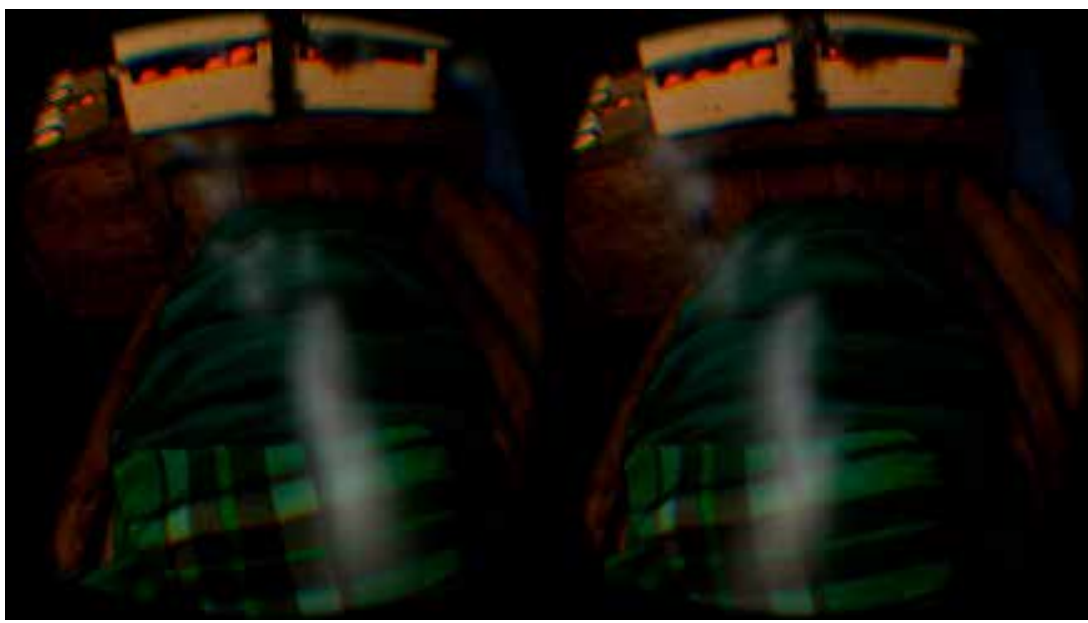

Figure 6.3

\section{Gameplay}

The game is divided into two scenes, which we will refer to as the dream scene (an auto-diegetic narrative introducing the protagonist's background) and the driving scene (a more interactive part representing the protagonist hidden in a truck), spanning five minutes in total. During the entire game, the player is free to move and look around within the physical boundaries of the contraption, while subtle visual elements are rendered in sync with the player's breathing by means of the anemometer (see Figure 6.3).

Although the game has a single script for the embedded narrative to provide each player with a similar experience, the player's choice of character determines the voice used for the auto-diegetic narration. In the dream scene, the player has no virtual body and floats mid-air in a dark room. An abstract movie is projected onto a white virtual wall, and an auto-diegetic voice recounts a raid kidnapping the protagonist's brother. Then the movie shows a boat drifting at sea, with the protagonist's voice explaining how he/she escaped the country, how the boat he/she was on sank not far from shore, leaving the protagonist as one of the few survivors. The movie transitions into the $3^{\mathrm{D}}$ surroundings as the player sees water rising from below to above his or her head (see Figure 6.4), as if being submerged and drowning, after which the environment turns pitch black, and the protagonist explains that continuing the journey through an overland route is the only option left.

The driving scene begins with the player waking up, hidden in a truck that is transporting mandarins (see Figure 6.5). 


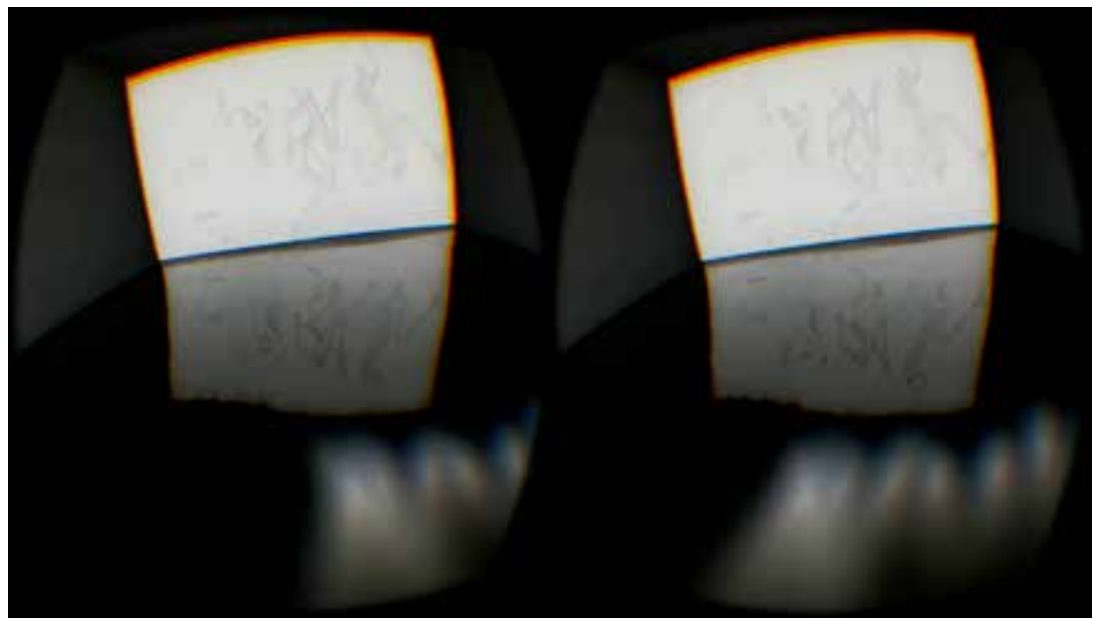

Figure 6.4

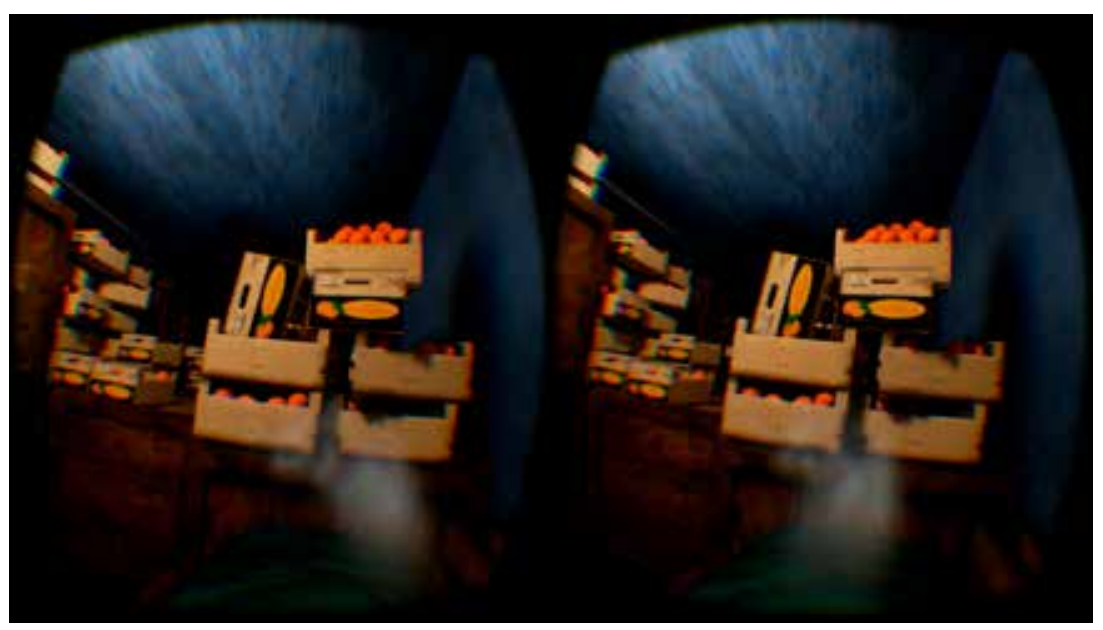

Figure 6.5

The diffusers in the respiration mask are activated and disperse the soothing scent of mandarins, as the unbalance motor starts generating a rocking motion that matches the movement of the virtual truck. A small window on the right side of the truck exists both in the physical and in the virtual world, and players can touch it and peek outside to see a Middle Eastern/ Balkan resembling scenery (see Figure 6.5).

After several minutes the truck is stopped, players hear a patrol questioning the driver, and an auto-diegetic voice tells the player to be quiet and not to breathe (which is actively monitored by the game system). The door on the right side of the truck swings open, the cargo is being 


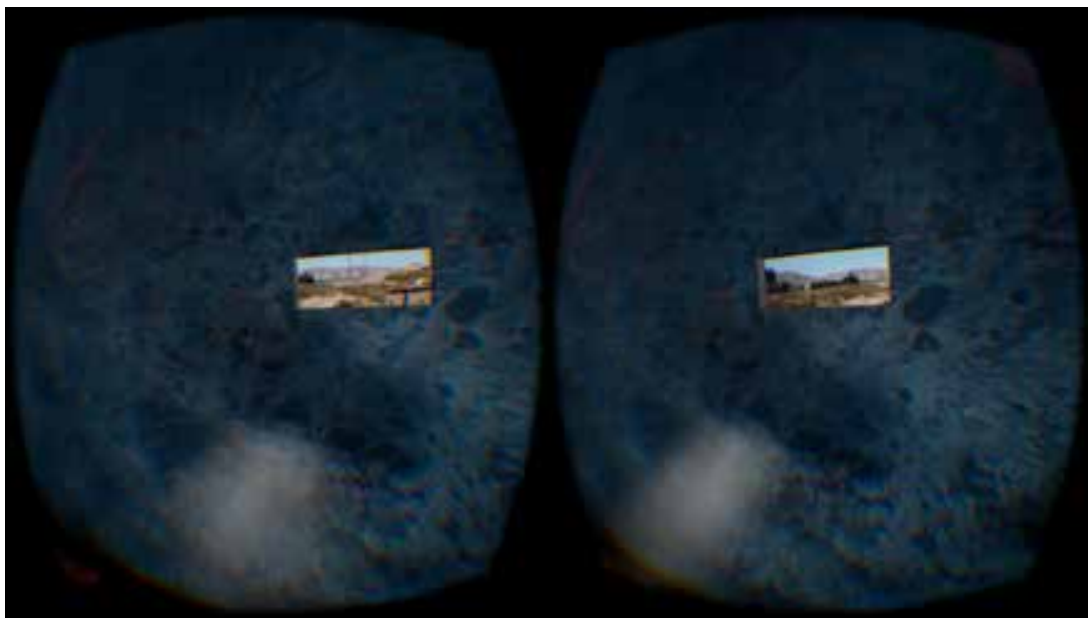

Figure 6.6

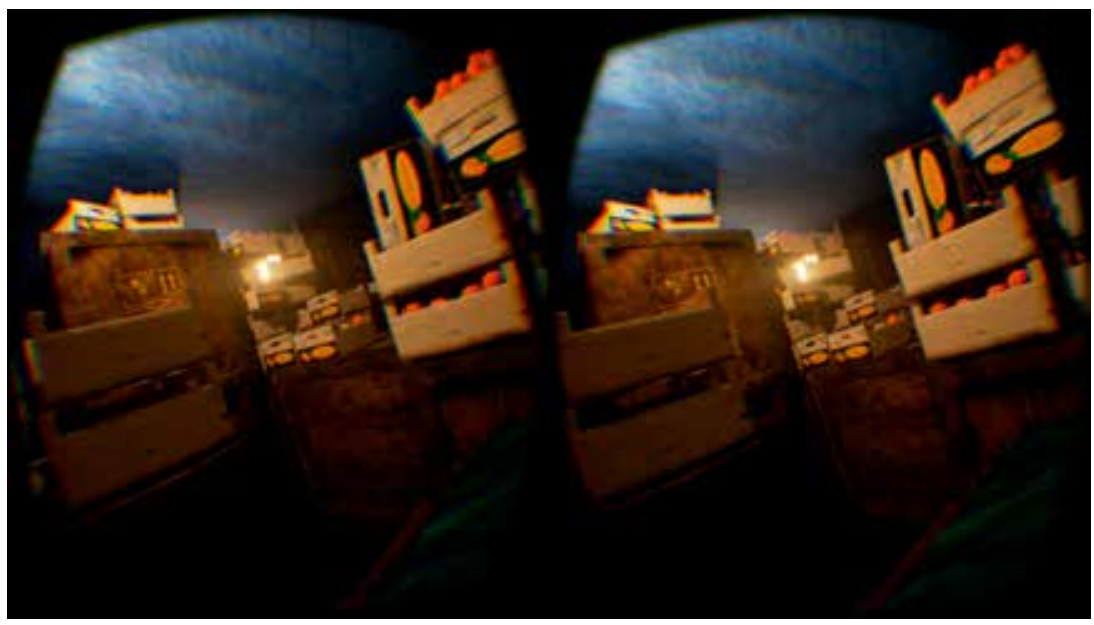

Figure 6.7

inspected, but nothing suspicious is found. When the door closes again several mandarins fall down (both in the virtual and physical world), alarming the patrol, who consequently inspects the truck a second time (see Figures 6.6 and 6.7).

This time, however, depending on whether the player is quiet and holding his/her breath, the system evaluates whether the protagonist is able to avoid detection or not. For this study, both endings are made nearly identical, fading the environment to gray, presenting the result of whether the player was caught or not, and ending with the auto-diegetic voice wishing a better future for those in a similar situation (see Figure 6.8). 


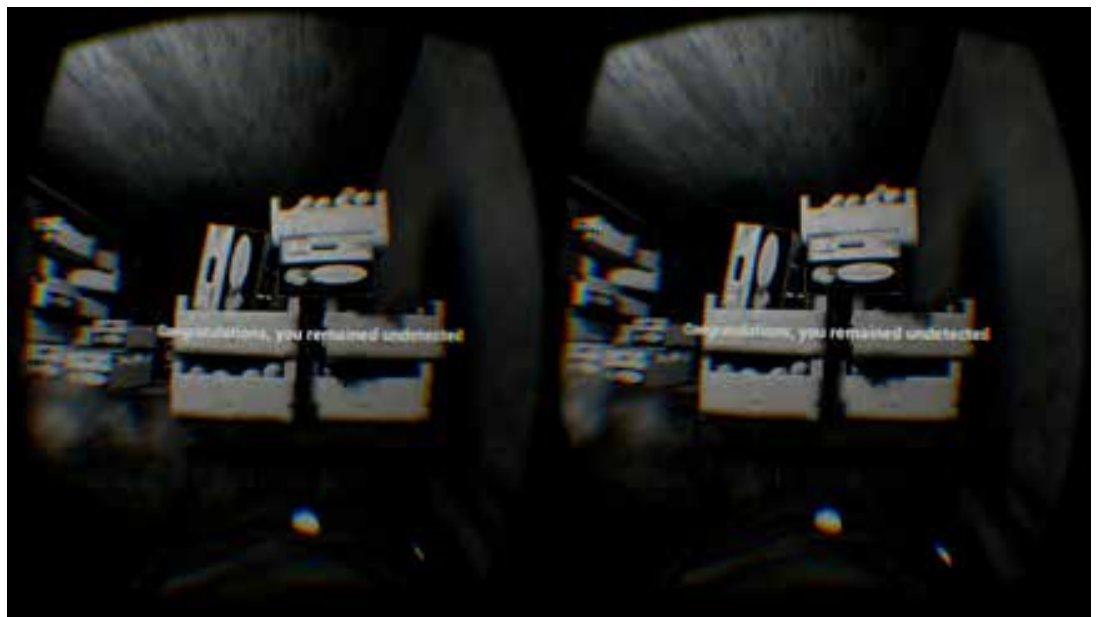

Figure 6.8

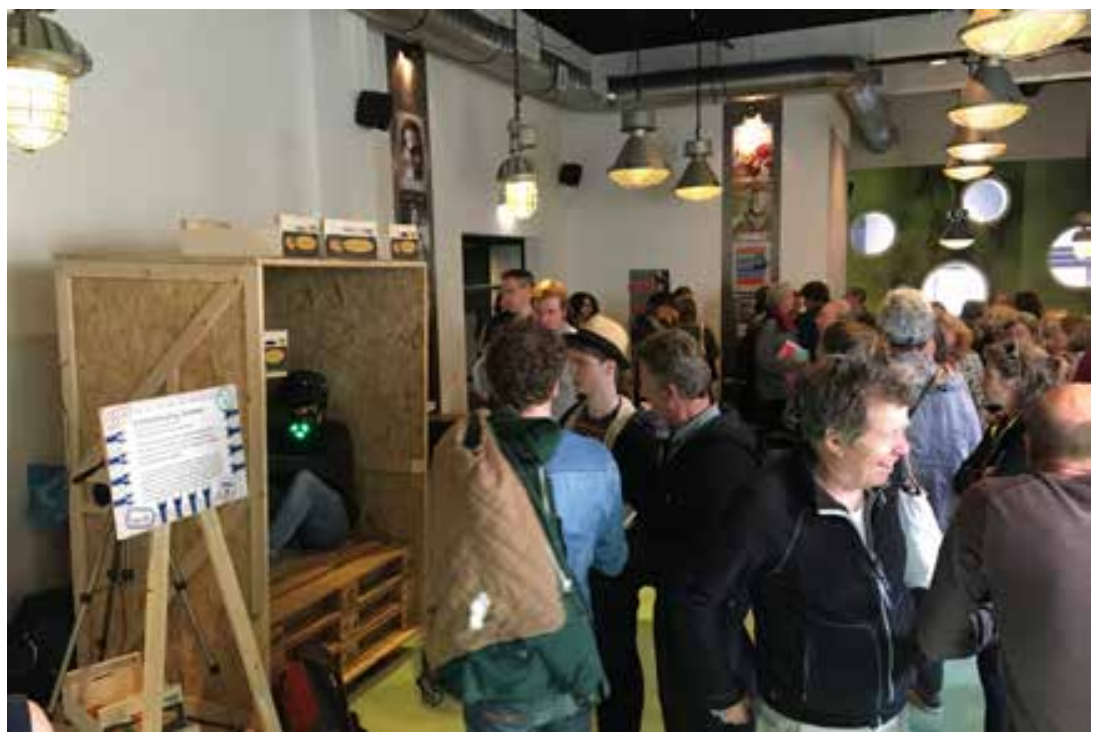

Figure 6.9

\section{Study}

ABTJ was deployed at three public events in Western Europe for a total of six days, between October and December of 2015 (see Figure 6.9). The objective was to probe reactions vis-à-vis empathy, embodiment, and persuasive appeals to inform the future design of empathic and persuasive experiences using system-immersion. 
We used an open-ended face-to-face interview protocol (Creswell, 2014) combined with a photo elicitation interview protocol in which participants were shown a diverse set of photo portraits of refugees and asked to choose one. In a short open-ended interview, they were asked to envision and tell a short narrative from the point of view of the character they selected, including events, contexts, thoughts, and feelings (Harper, 2002). Interviews were conducted either in English or in Dutch. The interview script aimed to obtain a better understanding of a participant's attitudes and empathy (or lack thereof) towards non-European migrants.

\section{Recruitment, grouping, and interview protocol}

Inclusion in this study required participants to be over 18 years old and to agree to be recorded during the interview. All participants included in the study were from Western European countries. We randomly created two groups: prospective participants were approached either when waiting in line before experiencing ABTJ (Group 1), or immediately afterwards (Group 2). Given the limitations of field studies (Presser et al., 2004), we were cautious not to prime participants before playing ABTJ and therefore decided to only interview participants either before (Group 1) or after (Group 2) playing ABTJ. Group 1 is our baseline group to assess the attitude towards refugees of people who had not experienced ABTJ. Group 2 actually played ABTJ. Their responses enabled us to qualitatively evaluate ABTJ's effects. A total of 70 people participated in the study. Group 1 had a total of 32 participants (13 females, 19 males) and Group 2 had 38 participants ( 13 females, 25 males).

After participants from Group 1 selected one of the six fictional characters (after being asked 'Whose journey do you want to experience?'), they were prompted to elaborate on the character's story, feelings, thoughts, and hopes (see Figure 6.10). The interview for Group 2 was identical, with modifications only to tenses (e.g., asking 'Whose journey did you just experience?'). We specifically inquired how the character would think and feel as a method to understand participants' self-expressed attitude from the perspective of a refugee. Their attitudes were collected both at the cognitive level of beliefs and the affective level of feelings.

We do acknowledge that a field setting might include greater distraction for participants, but it does offer a more natural setting as well, with greater realism compared to lab studies (Presser et al., 2004). Since participants were completely isolated from the environment during the experience, we found that the advantages of a field setting outweighed the disadvantages. Additionally, with a field setup we aimed for more accurate insights into 


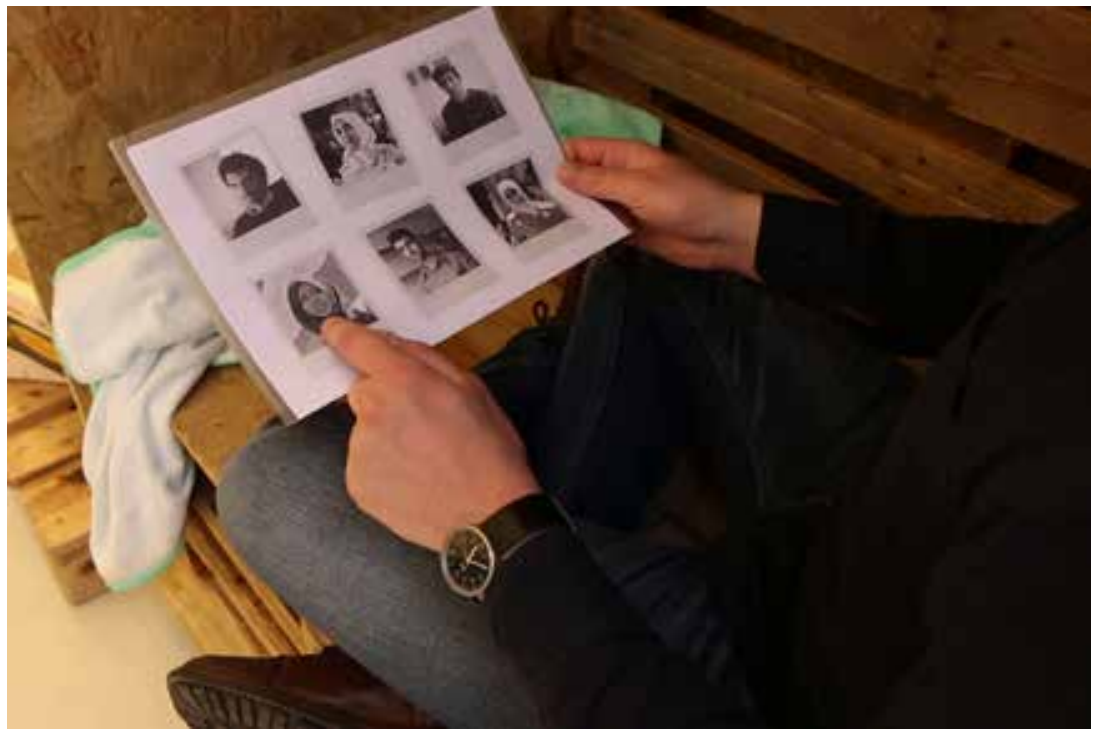

Figure 6.10

the future real-world application of empathy appeals (e.g., for NGOs and charities that promote their work at public events).

The open-ended interviews with the participants provided the empirical data for this study that were analyzed in a qualitative manner. Two coders categorized the data following Braun and Clarke's thematic analysis (2006). The interpretation of the data was also informed by narratological (Ryan, 2001) and pragmatist approaches (McCarthy \& Wright, 2004).

\section{Results}

The qualitative analysis resulted in four distinct themes: socially shared narrative schemas, post-hoc narrative interpolations, emotional markers, and embodied feelings. In this section, we will use the themes as lenses to present our participants' engagement with ABTJ and the consequences for their empathy with respect to refugees.

\section{Socially shared narrative schemas}

The topic of migration from Africa and the Middle East and of refugees escaping war-torn countries have been significantly present in Western media in the past decades, often becoming a polarizing topic in social and cultural discourses. Expressly leaving aside the political implications 
of these subjects, as a first step in our study we probed for spontaneous narratives about migrants from a group of our participants that had not yet interacted with ABTJ. With this first coding scheme, we teased out quotes from participants who produced coherent narratives about the experience of being a migrant without having interacted with ABTJ. Participants in Group 1 (interviewed before interacting with ABTJ) were shown a diverse set of photo portraits and asked to choose one. In a short open-ended interview, they were asked to envision and tell a short narrative from the point of view of the character they selected, including events, contexts, thoughts, and feelings. The participants themselves acknowledged the influence of mass media—as Peter (\#33) noted: 'This is of course [...] borrowed from the news, so I don't really have an idea about her on a personal level...'.

We observed some recurring narrative structures across these data-stories that, despite containing negative elements, were also about determination, willpower, and the fight for a better future. Emma (\#37) proposed: 'It looks like she is holding something. I think she is like a person who has demonstrated against something in her country, and uh, and therefore she is kind of strong, will power and because of that, as a consequence, she has to flee her country. She might have some children, and a husband. Maybe her husband is dead, and uh, her children might already be somewhere and she is protesting and wants to change something in the country she comes from.' In addition, Kevin (\#79) elaborated: 'I think he is about the same age as I am, so I think he is a student and I think he is looking for the best future [...]. He will seek this better future possibly in Europe or somewhere else where it's safe $[. .$.$] .'$

Whereas some participants also produced very short and generic descriptions, the examples proposed here show some articulated (albeit stereotypical) ones. There seems to be a recurring narrative schema at work here, arguably ingrained in some mainstream media representation of migrants and refugees. Thematic nuclei in these archetypical structures include the escape from a war-torn country to avoid violence or persecution; embarking on a long, difficult, and stressful journey trying to reach a safe haven; worries about what the future might bring; and having to leave family behind in the process. Let us now return to the embedded narrative programmed into ABTJ: for the purpose of our study, that storyline was designed to be as generic as possible, without deviations from a canon of refugee stories. It mentions the protagonist's brother's abduction, the decision to flee the country, a dangerous journey by sea and a ride in the back of truck, hiding in a small space between crates-all elements that fit into the socially shared narrative schema outline above. 


\section{Narrative interpolation}

Narrative interpolation, that is, the creation of a story-like account of firstperson experiences, occurred generally in relation to parts that clearly refer to the diegetic world represented in ABTJ but at the same time do not exist in the embedded narrative. Following the same protocol of Group 1, participants in Group 2 (interviewed after interacting with ABTJ) were shown photo portraits, asked to choose one, and told that the person depicted was also the one they enacted during the experience. Then, they were asked to tell a short narrative from the point of view of the selected character. Mark (\#47) gave the following interpretation which was not literally and immediately connected to the embedded narrative: 'You make yourself very, very small, very insignificant, you sit in a corner behind boxes, your window is very small.' Daniel (\#31) related how he would feel in the same situation: 'Well, I guess that I felt how anxious it is to endure the journey, and constantly on the lookout to avoid being discovered, be sent back, or murdered [...] Especially when it [the truck] was stopped [...] And when the door really opened, it started to feel quite tense. It was the moment I was also pointed out to hold my breath when I thought, oh yeah, then I was suddenly totally pulled into the experience, oh yes, this is how it is, quite exciting what's happening here. [...] I really thought, oh this is the moment that I should really remain quiet because otherwise I might soon be discovered.'

These quotes demonstrate how our participants interpolated the narrative they experienced by adding details not originally present in the embedded script. Neither the narrating voice of ABTJ nor the on-screen action depicts the protagonist making himself/herself 'very, very small' (Mark) or 'being constantly on the lookout' or '[starting] to feel quite tense' (Daniel). And yet these interpolations do make sense within the overall narrative, and the two participants were arguably using them as a means to interpret and convey their own personal experience with ABTJ.

\section{Emotional markers}

Our participants used a broad variety of linguistic markers to indicate emotions within the narrative interpolations of their ABTJ experience. To examine them in more detail, we made a first broad pass coding emotional statements utilizing Power and Dalgleish's factor model for correlated basic emotions (2008). The following quotes present some examples of emotional markers we teased out from within parts that are clearly narrative interpolations. On the one hand, we observe a broad palette of markers ranging from 
simple to ones that are more complex. For example, Emily (\#12) reported: '[it was] very exciting, tense, especially, yes, you especially are very vigilant, and pay attention. I did not quite know where [the character] came from.' Frank (\#4) mentioned an ambiguous condition: 'Um. She was sad but also happy. She was the only survivor of a ship which sunk. And then she was really just very anxious, and especially when she was almost discovered.' Other participants similarly used the words 'anxious' and 'tense' (Benjamin) or 'scared' (Brian, Anna, and Sarah), 'afraid', and 'nervous' (Arthur). In addition to this, we also observed other more nuanced expressions that convey an emotional connotation coupled with other components that are rooted in physical and/or narrative contexts, such as 'But the [interesting] experience is especially the part when you are locked up in that truck and that you are in her skin. Smell helps, and I felt that I was there, found that it works well' (Linda) or 'you feel trapped' (Paul).

\section{Embodied feelings}

Throughout the narrative interpolations produced by our participants, we observed an abundance of expressions pointing to one's own body, to body parts, and to bodily characteristics. This prompted us to apply another specific coding to tease out how our participants leveraged embodied terms and expressions to convey specific feelings, emotions, and perceptions more accurately.

A first recurring code involving embodiment is the sense of being physically constricted, almost to the point of claustrophobia. Susan (\#4) mentioned: 'If you are sitting there [note: hidden in a truck] for a whole day it should be awful, I never really realized, I never, it feels quite small, claustrophobic. [...] I didn't really feel fear, it was more desperation.' Others talked about being 'locked up [...] and [...] in her skin' (Linda), 'your contact with the outside world is minimal. Uh, yeah, and you feel trapped' (Paul), and 'you realize how lonely one must feel [...] this confined perspective' (Lily).

Different participants elaborated on being prompted by the installation to control their breathing, such as 'It was the moment I was also pointed out to hold my breath when I thought, oh yeah, I was suddenly totally pulled into the experience, oh yes, this is how it is, quite exciting what's happening here' (Daniel). But breathing was also pointed out to be something on which the device and the embedded story have an indirect effect: for example, Emily referred to the scene in which the protagonist is getting submerged in water and reports that '[in] the water, you feel some kind of short[ness] of breath'-it should be mentioned that the device worn by users was not 
actively restricting their airflow in any way. The emphasis that participants placed on bodily feelings and on physical sensations in their recounting of the experience is significant. It suggests how a first-person mixed-reality experience may provide players with a repertoire of embodied feelingseven those not explicitly mentioned in the embedded narrative-over which they may elaborate.

The four themes and their related codes and observations that emerged from our exploratory study are partially surprising. We found evidence of participants clearly situating their experience in the contemporary massmedia discourse (Theme 1: socially shared narrative schemas) and yet being willing to interpreting freely and adding to the embedded narrative (Theme 2: narrative interpolation). This suggests not only a promising level of engagement but also the use of the immersive experience as an activator, some kind of a catalyst that users leverage to tell original stories about migrants. The third and fourth themes (emotional markers and embodied feelings) are interesting for their distribution, as they often co-occur in our dataset. This suggests that emotions and physical stimuli-real or simulated-may mutually influence and reinforce each other. We will now extrapolate three design opportunities and related concepts supported from our RtD process, deployment, and analyses that we offer to the design community as an input for future work.

\section{Design opportunities}

Following our observations and analysis, we can discern three different game design opportunities to stimulate empathy arousal in mixed reality. These are visceral engagement, moments of reflection, and affective appeals.

\section{Visceral engagement}

The concept of viscerality has already been explored in HCI design, as exemplified by Norman (2005) who describes the visceral level of an artifact as its look, feel, sound, and, more generally, its material components as they support and orient its intended functions. He argues that humans 'are exquisitely attuned to receive powerful emotional signals from the environment that get interpreted automatically at the visceral level' (Norman, 2005). Benford et al. (2012) and Byrne, Marshall, \& Mueller (2016) have already begun exploring aesthetic experiences produced by stimuli acting on users' bodily core, such as breathing, sweating (Benford et al., 2012), and 
vertigo (Byrne et al., 2016). Indeed, the data we collected demonstrate how important the bodily, or visceral, experience was. This is clearly still a much underexplored design space, whose study has been made timely by the rapid diffusion of immersive, tangible, and wearable technologies. The vertigo caused by the fear of heights, the uneasiness of gazing into a stranger's eyes, the sense of weightlessness and breathlessness in a rollercoaster are good examples of the opportunity space that can be realized with contemporary accessible technology. In ABTJ, two moments of visceral engagement stood out, notably, the part where the user is submerged in water and the part where the player sits between crates, looking outside, while the truck is moving. Both parts provide multi-sensory experiences that go beyond the audio-visual (the proprioceptive feelings of breathing and of being constricted between crates), that have an inward directionality (inhalation, pressure), and are uncomfortable - if not threatening - to the user's avatar. For example, a participant said she experienced a shortness of breath when visually submersed in water, even though the mask did not restrict airflow. The other moment, when sitting in the truck, participants presented experiences that relate to feeling trapped, cramped, and claustrophobic, some explaining that this made them feel very small and insignificant. Being able to create these intense moments provides a compelling case for using system-immersion, and in particular multi-sensory/embodied mixed-reality experiences, to explore complex feelings that are otherwise difficult to convey.

Although visceral engagement remains an underexplored area, we propose that one can stimulate visceral reactions by taking away players' agency of bodily freedom, creating a sense of confinement through physical space and objects, or reacting to biofeedback during gameplay, for example analyzing the players' breathing to determine game progression. Discomfort and suspense are another aspect to consider.

\section{A moment of reflection}

Mixed-reality experiences offer the possibility of sensing and feeling not only the experience of being somewhere else but also the experience of inhabiting someone else's mind. Temporarily inhabiting other people's perspectives, sensing what they sense, and potentially feeling what they feel would seem to address most characteristics required for an empathic experience (Wiseman, 1996). However, this raises a fundamental question: when we adopt someone else's point of view through system-immersion, with whom are we empathizing? With the person whose mind we temporarily 
inhabit? With other people entering our field of view? Clouds Over Sidra (Arora et al., 2015), whose gameplay has been described as 'you're sitting there in her room, $[. .$.$] when you look down, you're sitting on the same$ ground that she's sitting on' (Milk, 2015, para.13), clearly favors this second opportunity. We wonder whether there might be more potential in systemimmersion than what we are using now: can players empathize with their avatars? With ABTJ, an essentially solitary experience, we have explored this specific opportunity. And yet, an issue surfaces: if empathizing is the process by which one understands nonjudgmentally the experiences, the feelings, and the difficulties of another person, in particular in the states of empathic concern (i.e., 'feeling for another person who is in need') or imagine-other perspective (i.e., 'imagining how another person thinks or feels given his/her situation.') (Batson \& Ahmad, 2009), then this is rendered more complicated by a first-person perspective that does not immediately provide the participants with someone to empathize with other than with oneself. Or, in Bob's words, 'I think I played as myself.' This process would require reflection and introspection in order to allow users to separate themselves from the character whom they are asked to empathize with. However, reflecting in games is non-trivial, because creating a rich experience leads to a flow paradox in which being more involved with gameplay can lead to less critical reflection (Squire, 2005). Does this mean that it is unlikely for a person to have an immersive and interactive first-person empathic experience that fosters empathy towards another? On the contrary, as a future design opportunity, we point out the empathic relationship with one's own avatar as a still understudied possibility to examine. As a prerequisite, we emphasize the usefulness of allowing participants a moment of reflection upon finishing the game. We argue that this would give them the chance to use the material they experienced to empathize with the other. In our study, this chance seemed actively evoked through the post-questionnaire. In lieu of a formal debriefing, we also found that some participants spontaneously used the two minutes of ABTJ when the protagonist just sits in the moving truck without any told narrative or challenges as an opportunity to reflect. Some participants reported that this moment offered a chance for them to drift away in their thoughts, almost like a mindful experience. This might have actually also presented a moment of reflection and deeper processing: a moment in which participants had the time to relate their virtual experiences to someone else's experiences in reality, realizing how lonely and claustrophobic such a journey would feel for a refugee. We underline how the majority of narrative expansions and reported visceral reactions took place in that specific moment. We therefore 
call on designers to create pockets of downtime in which people cannot (or-more preferably — do not wish to) do anything else than 'experience' (Kors, van der Spek, Ferri, \& Schouten, 2016).

\section{Affective appeals}

About a decade ago, Bogost argued that games have unique persuasive powers mainly due to their capacity to make arguments through their rules and procedures (Bogost, 2007). In line with scholars who have pointed out that games can also use other dimensions to shape attitudes (De la Hera, 2019), the rapid diffusion of system-immersion might bring an additional — more emotionally engaging — aspect that is particularly suitable for games, which is the experience of a fully mediated presence. Our analysis of ABTJ suggests multiple ways in which designers can use mixed reality to strengthen the affective appeal of their game, in particular through embodied multi-sensory experiences. For future designs, we would draw attention to the use of additional factors beyond immersive audiovisuals to increase presence, including the use of scents as a means of pulling players into the experience. Furthermore, affective appeals supported by system-immersion might particularly benefit from leveraging a sense of fear or anxiety as ways to set up a type of persuasive argument, for example by appealing to the experience of claustrophobia. However, with highly immersive experiences, frightening experiences can become overwhelming and perhaps cause distress. We therefore urge designers to be careful when leveraging system-immersion to evoke negative emotions.

\section{Conclusions}

With the recent rise of system-immersion (virtual and mixed reality), many questions have surfaced on the potential use of system-immersion to influence how we think and feel about reality, with in particular the captivating potential to foster empathy by having players inhabit another person's perspective. But despite the strong interest from industry, where virtual reality is already labeled as the 'ultimate empathy machine' (Milk, 2015, para.6), little research has actually focused on the arousal of empathy through immersive experiences and its subsequent value for persuasion. Following a Research through Design approach, we created A Breathtaking Journey, a multi-sensory mixed-reality game that provides the player with a first-person perspective of a refugee's journey. A qualitative study was 
conducted to tease out empathy-arousing characteristics and to chart this novel design space. We observed reactions coherent with the characteristics of empathic responses and formulated three design opportunities-stimulating visceral reactions, introducing moments of reflection, and leveraging affective appeals - for further applications and research in this field.

The first design opportunity relates to the stimulation of viscerally engaging experiences. Our study and related literature point to a design space characterized by visceral feelings that are insuppressible, intense, and difficult to label but are felt deep inside. These feelings are difficult to mediate through legacy media and seem to require an elevated sense of presence. The second opportunity space relates to a moment of reflection. System-immersion can support the temporary inhabiting of another person's perspective, sensing what they sense and feeling what they feel. Such a first-person perspective, however, complicates other-oriented empathic states, as it does not immediately provide the player with someone to empathize with other than with oneself. Our study points out a way to overcome this issue by introducing a moment of reflection through the inclusion of downtime to temporarily slow down the pace of the experience and offer players a mindful moment to acknowledge the other person they are inhabiting. The third opportunity relates to the affective appeals of system-immersion. In our study, we found that participants primarily embrace emotions rather than logical argument, which is a clear indication that system-immersion is able to convey complex emotional experiences such as loneliness and insignificance quite effectively.

There is clearly much more research and design work to do regarding empathy arousal in games. We see a still untapped potential for playful persuasive interaction, which includes games that could benefit from the exemplary prototype of ABTJ and the three design opportunities presented in this chapter. In this vein, we call for game designers to consider the emerging opportunities of empathy as a persuasive appeal in the design of persuasive games.

\section{References}

11 bit studios (2014). This War of Mine [Digital Game].

Antonisse, J., \& Johnson, D. (2013). Hush [Online Game].

Arora, G., Pousman, B., \& Milk, C. (2015). Clouds Over Sidra [VR Film].

Bartelson, E. (2013). Making Violence Meaningful Through Narrative in Spec

Ops: The Line. Retrieved 20 July 2019 from http://ctrl500.com/game-design/ making-violence-meaningful-through-narrative-in-spec-ops-the-line. 
Batson, C.D., \& Ahmad, N.Y. (2009). Using Empathy to Improve Intergroup Attitudes and Relations. Social Issues and Policy Review, 3(1), 141-177. doi:10.1111/j.17512409.2009.01013.x.

Belman, J., \& Flanagan, M. (2010). Designing Games to Foster Empathy. International Journal of Cognitive Technology, 14(2), 11. Retrieved 9 May 2018 from https:// tiltfactor.org/wp-content/uploads2/cog-tech-si-g4g-article-1-belman-andflanagan-designing-games-to-foster-empathy.pdf.

Benford, S., Greenhalgh, C., Giannachi, G., Walker, B., Marshall, J., \& Rodden, T. (2012). Uncomfortable Interactions. Proceedings of the SIGCHI Conference on Human Factors in Computing Systems CHI '12, 2005-2014. doi:10.1145/ 2207676.2208347 .

Bogost, I. (2007). Persuasive Games: The Expressive Power of Videogames. Cambridge, MA: MIT Press.

Boltz, L.O., Henriksen, D., \& Mishra, P. (2015). Rethinking Technology \& Creativity in the 21st Century: Empathy through Gaming-Perspective Taking in a Complex World. TechTrends, 59(6), 3-8. doi:10.1007/s11528-015-0895-1.

Braun, V., \& Clarke, V. (2006). Using Thematic Analysis in Psychology. Qual. Res. Psychol., 3(2), 77-101. doi:10.1191/1478088706qpo63oa.

Brown, J., Gerling, K., Dickinson, P., \& Kirman, B. (2015). Dead Fun: Uncomfortable Interactions in a Virtual Reality Game for Coffins. Proceedings of the $2015 \mathrm{An}$ nual Symposium on Computer-Human Interaction in Play CHI PLAY' $15,475-480$. doi:10.1145/2793107.2810307.

Byrne, R., Marshall, J., \& Mueller, F. F. (2016). Designing the Vertigo Experience: Vertigo as a Design Resource for Digital Bodily Play. Proceedings of the TEI ' 6 : Tenth International Conference on Tangible, Embedded, and Embodied Interaction TEI '76, 296-303. doi:10.1145/2839462.2839465.

Carpenter, J.M., \& Green, M.C. (2012). Flying with Icarus: Narrative Transportation and the Persuasiveness of Entertainment. In L.J. Shrum (ed.), The Psychology of entertainment media, pp. 169-194. New York, NY: Routledge.

Creswell, J. (2014). Research design: Qualitative, quantitative, and mixed methods approaches. California: SAGE.

De la Hera, T. (2019). Digital Gaming and the Advertising Landscape. Amsterdam: Amsterdam University Press.

Se la Peña, N., Weil, P., Llobera, J., Giannopoulos, E., Pomés, A., Spanlang, B., Slater, M. (2010). Immersive Journalism: Immersive Virtual Reality for the First-Person Experience of News. Presence: Teleoperators and Virtual Environments, 19(4), 291-301. doi:10.1162/PRES_a_00005.

De Smale, S., Kors, M., \& Sandovar, A. (2017). The Case of This War of Mine: A Production Studies Perspective on Moral Game Design. Games and Culture, 14(4), 387-409, doi:10.1177/1555412017725996. 
Dinh, H.Q., Walker, N., Hodges, L.F., Song, C., \& Kobayashi, A. (1999). Evaluating the Importance of Multi-Sensory Input on Memory and the Sense of Presence in Virtual Environments. Virtual Reality, 1999. Proceedings., IEEE, 222-228. doi:10.1109/VR.1999.756955.

Fiore, S., Wright, P., \& Edwards, A. (2005). A Pragmatist Aesthetics Approach to the Design of a Technological Artefact. Proceedings of the 4 th Decennial Conference on Critical Computing: Between Sense and Sensibility CC '05, 129-132. doi:10.1145/1094562.1094581.

Harper, D. (2002). Talking About Pictures: A Case for Photo Elicitation. Visual Studies, 17(1), 13-26. doi:10.1080/14725860220137345.

ImpactGames. (2007). PeaceMaker [Online Game].

Jenkins, H. (2004). Game Design as Narrative Architecture. Computer, 44(3), 118-130. Retrieved from http://www.madwomb.com/tutorials/gamedesign/ Theory_HenryJenkins_GameDesignNarrativeArchitecture.pdf.

Kors, M., Van der Spek, E., \& Schouten, B. (2015). A Foundation for the Persuasive Gameplay Experience. Proceedings of the 1oth Foundations of Digital Games Conference. Retrieved 14 July 2019 from https://dblp.org/rec/conf/fdg/KorsSS15.html. , van der Spek, E.D., Ferri, G., \& Schouten, B.A.M. (2016). Occasionally a Dull Moment. Designing for Introspection and Retrospection in Persuasive Games. DiGRA 2016 Workshop:Morality Play: The Design of Games for Moral Engagement. Lankoski, P. (2007). Goals, affects, and empathy in games. In Sageng, J., Fossheim, H. \& Larsen, T. (eds.), The Philosophy of Computer Games. New York, NY: Springer. Lucero, A., Karapanos, E., Arrasvuori, J., \& Korhonen, H. (2014). Playful or Gameful?: Creating Delightful User Experiences. Interactions, 21(3), 34-39. doi:10.1145/2590973.

Marshall, J., Walker, B., Benford, S., Tomlinson, G., Rennick Egglestone, S., Reeves, S., Longhurst, J. (2011). The Gas Mask: A Probe for Exploring Fearsome Interactions. CHI' 1 Extended Abstracts on Human Factors in Computing Systems CHI EA '11, 127-136. doi:10.1145/1979742.19796o9.

McCarthy, J., \& Wright, P. (2004). Technology As Experience. Interactions, 11(5), 42-43. doi:10.1145/1015530.1015549.

Milk, C. (2015). How Virtual Reality Can Create the Ultimate Empathy Machine. TED Talks. Retrieved 15 May 2019 from https://www.ted.com/talks/ chris_milk_how_virtual_reality_can_create_the_ultimate_empathy_machine/ transcript.

Norman, D.A. (2005). Emotional Design: Why We Love (Or Hate) Everyday Things. California: Nielsen Norman Group.

Peng, W., Lee, M., \& Heeter, C. (2010). The Effects of a Serious Game on RoleTaking and Willingness to Help.Journal of Communication, 6o(4), 723-742. doi: 10.1111/j.1460-2466.2010.01511.x. 
Power, M., \& Dalgleish, T. (2008). Cognition and Emotion: From Order to Disorder (2nd ed.). Brighton: Psychology Press.

Presser, S., Rothgeb, J.M., Couper, M.P., Lessler, J.T., Martin, E., Martin, J., \& Singer, E. (2004). Methods for Testing and Evaluating Survey Questionnaires. Hoboken, NJ: John Wiley \& Sons.

Ryan, M.-L. (2001). Narrative As Virtual Reality: Immersion and Interactivity in Literature and Electronic Media. Baltimore, MD: Johns Hopkins University Press.

Schouten, B.A.M., Deen, M., \& Bekker, M.M. (2010). Playful Identity in Game Design and Open-Ended Play. Proceedings of the Homo Ludens Conference. Retrieved 28 October 2018 from https://www.jstor.org/stable/j.ctt14brqd4.8.

Squire, K.D. (2005). Educating the Fighter: Buttonmashing, Seeing, Being. On The Horizon - The Strategic Planning Resource for Education Professionals, 13(2), 75-88. doi:10.1108/10748120510608106.

Wiseman, T. (1996). A Concept Analysis of Empathy.Journal of Advanced Nursery, 23(6), 1162-1167. doi: 10.1046/j.1365-2648.1996.12213.x.

Zimmerman, J., Forlizzi, J., \& Evenson, S. (2007). Research Through Design As a Method for Interaction Design Research in HCI. Proceedings of the SIGCHI Conference on Human Factors in Computing Systems CHI 'o7, 493-502. doi:10.1145/1240624.1240704.

\section{About the authors}

Martijn Kors is a doctoral candidate at the Department of Industrial Design of the Eindhoven University of Technology in Eindhoven, the Netherlands. His design-oriented research focuses on studying the intersections between persuasive game production, system-immersion, and the employment of empathy-arousing appeals to shape how players think and feel about issues in reality.

Gabriele Ferri is Associate Professor in Urban Interaction Design at the Amsterdam University of Applied Sciences as well as senior researcher at the lectorate in Play \& Civic Media at the same institution. His research interests include values in design and design ethics, speculative design as a tool for empowerment, qualitative research methods in interaction design, and critical uses of game design.

Erik D. van der Spek is Assistant Professor of Game and Play Design at the Eindhoven University of Technology where he leads the ID Play Lab. His research interests lie in the design of games and play for entertainment, 
learning, vitality, and empathy. He also founded the game design consultancy firm Game Descience in 2013.

Cas Ketel graduated in 2015 from the Department of Industrial Design at the Eindhoven University of Technology with a virtual reality refugee experience called A Breathtaking Journey. He is a professional designer who develops virtual reality (VR) and augmented reality (AR) products ranging from training tools and interactive game experiences to visualizations and events.

Ben Schouten is an artist/designer as well as a Full Professor in playful interaction at the Department of Industrial Design of the Eindhoven University of Technology. His research focuses on play and participatory design for social innovations and citizen empowerment through bottom-up approaches. He has co-edited five volumes and conference proceedings and has co-authored more than 100 publications. 


\title{
7. Macro, Micro, and Meta-Persuasive Play to Change Society
}

\author{
Lindsay D. Grace
}

\begin{abstract}
This chapter examines persuasive games through the dominant arguments made about the value of such designed play. Beyond the who, what, and where, there is the why. Why do researchers and practitioners want to persuade people through games? Why are games the right-or potentially wrong - medium for delivering persuasive messages? Why has public discourse come to need games as a vehicle for communicating and argumentation? Why has the design of such play grown into an increasingly media-rich environment that is seemingly adrift, unable to decant the real from its opposite?
\end{abstract}

Keywords: macro-persuasions; micro-persuasions; meta-persuasions; gamification; industry; play rhetoric

\section{Introduction}

This chapter examines persuasive games through the dominant arguments made about the value of such designed play. Beyond the who, what, and where, there is the why. Why do researchers and practitioners want to persuade people through games? Why are games the right —or potentially wrong - medium for delivering persuasive messages? Why has public discourse come to need games as a vehicle for communicating and argumentation? Why has the design of such play grown into an increasingly media-rich environment that is seemingly adrift, unable to decant the real from the unreal?

These questions are not the mere product of diegetic examination. Instead, they are drawn from the daily operations of directing a game studio at

Hera, T. Dela, J. Jansz, J. Raessens, B. Schouten, Persuasive Gaming in Context. Amsterdam: Amsterdam University Press, 2021 DOI 10.5117/9789463728805_CHO7 
the apex of the increasingly complicated political theater of Washington, DC, its interplay with democracy, and the dissemination of information. These questions are informed by contracted work and practiced research in purpose-driven games. This work is carried out for a variety of professional clients that include a number of the Smithsonian Museums, the World Bank, the International Finance Corporation (IFC), Education Testing Services (ETS), the U.S. National Institutes of Mental Health (NIMH), and several radio, television, and news organizations. The questions also relate to work to improve journalism through game design (Grace \& Farley, 2016) and projects for public and private media organizations.

In 2013, the American University Game Lab was founded with the mission to research, teach, and practice persuasive play. There are now several definitions of persuasive play and persuasive games. Bogost's version is the most commonly used (Bogost, 2007), but I prefer a marketing-inspired definition of persuasive play as games and other engagement strategies designed to change a player's interests, activities, or opinions. This definition converts the lifestyle segmentation used by demographers and market researchers (Plummer, 1974) toward games. It also carries a longer running tradition of assessment and efficacy analysis informed by more than 30 years of demographic and psychographic research. In the array of demographer tools, for instance, there are methods for understanding the current state of people's interests, activities, and opinions (Demby, 2011). Assessing the efficacy of any persuasive play engagement can proceed from gauging the pre-persuasive play and postpersuasive play state of those three attributes. Admittedly, these definitions are pragmatic and lack the academic inspiration of Bogost's seminal work.

I propose that there are three broad categories of persuasive play that are worthy not only of research but as foci for industrial practice. These are: macro-persuasions, micro-persuasions. and meta-persuasions. Macropersuasions are the efforts across gaming made about games and their relationship to society. Micro-persuasions are the games themselves that aim to employ persuasive play. Meta-persuasions, meanwhile, are the efforts, whether playful or nefarious, that act upon non-play systems as though they were systems. Meta-persuasions are the least obvious of the three, originating partly as the byproduct of macro and micro-persuasions, in part due to the absence of critical examination, and most obviously as the application of playful thinking to non-playful contexts. If purposeful games, persuasive games, or the much-debated serious games are applications of games in non-game contexts, meta-persuasions are their complement. Meta-persuasion is the application of play in non-play contexts, and it works against big data by producing big bad data (aka poorly constructed or fake 
data). It also works against the democratic function of real news by producing fake news or playing devil's advocate where no such advocate is required.

The characteristics of persuasive gaming preface each of these efforts. There is, for example, procedural rhetoric in macro and meta-persuasive levels and micro-persuasive games. The results of these persuasions do yield a byproduct, namely the unintended persuasion. Through a combination of case-study reports and position setting, this chapter outlines the proof, character, and exemplars of each of these persuasions.

The chapter focuses on macro-persuasion as a case study in how largescale efforts to change the interests, activities, and opinions of game players and non-players have been executed over the past decade. These efforts directly affect all of the persuasions. Macro-persuasions demonstrate a kind of application of persuasive play design in non-game contexts. They are not an application of gamification but instead a chorus of efforts designed to elevate the status and efficacy of games in general society.

The goals of this chapter are to help others in the persuasive games space examine these practices to refine their own work and develop appropriate strategies for combating the unintended results of such work, and to provide a topographic view of how such strategies might be applied elsewhere.

\section{The macro-persuasion}

There are several ways to frame persuasive games. At the macro level, they can be framed as a whole. This whole is comprised of the creative product of games, the practice of playing games, and the communities that orbit games. In this framework, games are a quantifiable, distinct, and discrete set. To make sense of this, games must be viewed as an artifact, and viewing them in this way requires framing them distinctly from one or more of the following lenses:

- Games are the artifact of game design and development; or

- Games are a cultural artifact of leisure; or

- Games are the creative artifact of contemporary expression; or

- Games are the artifact with which players interact.

A game as an artifact is supported by these or any of the other ways in which games can be described as a product not of a commercial system but of humanity. Being an artifact of humanity or humanness focuses its value and the claims thereto. 
The macro-persuasions are centered on making claims about games in general. Often, such persuasions contain arguments made for the inherent value - or lack of value - of games. This includes organizations that aim to legitimize the cultural capital and significance of games, game-makers, and their players. In the past decade, a chorus has grown to persuade the world that games are more than 'mere' entertainment, with supporters typically championing the value of games as cultural artifacts (Greenfield, 1994) and as a means to support and form a community (Squire, 2011), foster citizen participation (De Lange, 2015), express creativity (Jenkins, 2005), or practice desired skills (Gee, 2004).

This is not a new persuasive aim, but it is one that persists and for which the scale has grown. The hallmarks of this effort include everything from the Games for Change Festival (http://www.gamesforchange.org/), the many serious games conferences that take place, and the myriad of conventions, events, and other gatherings that aim to argue in favor of the value of the medium. They include the Institute of Play Inc. (https://www.instituteofplay. org/), the Institute for Play (http://www.nifplay.org/), the Higher Education Video Game Alliance (HEVGA), and others. These organizations and their efforts have proliferated in the years since Bogost first wrote his book, Persuasive Games (2007).

The impact of these organizations' efforts is arguably less significant than the effect of their mere existence. Regardless of how many awards are given, how many papers are published, or how many people convene to support the organizational efforts, the ability to hoist the banner of ten-year-old organizations and large conference attendances is clearly noteworthy. It is capable, though, of obscuring the relatively limited identifiable successes within persuasive play.

The mere fact that the Games for Change Festival, for example, has maintained audiences of at least 300 for more than a decade clearly emphasizes significant and persistent support for games of this type. The support of major private foundations, industry partners, and the public only further enhances the claims of the potential for such games. In the grand scheme of macro-persuasion, it means little that serious games, for example, continue to lack definition or widespread adoption. It is, instead, such a rhetoric-rich environment that much of the evidence is built more on the idea than the reality, which is a theme that has overshadowed a variety of major gatherings but has never dampened spirits or enthusiasm. Like all game players, we are unsure whether what we are doing is working, but we are working to do it all the same. Similar to learning how a game works, some action is often better than no action. Of course, the evidence of the more than 140 research 
studies published on such work demonstrates positive behavior change and outcomes (Boyle et al., 2016), meaning that there are grounds for optimism.

\section{Increasing the cultural capital of games}

Whether or not games have risen from the often-disposable world of popular culture to the more elite space of cultural contribution is arguable. We know, for example, that games have some cultural capital by being recognized at museums (Grace, 2017) and other respected institutions that offer markers of cultural worth. What is perhaps more important is to understand how this increase in reputation has occurred and at what cost.

There were few game-art exhibits of international note in 2007, but in 2017 there were 22 large-scale events offering games as culturally valuable and not just as industrial products of historical novelty. The growth can be attributed to the past success and an increased appetite for the genre. The rise in geek culture (King et al., 2003), the continued value of technical fields (aka STEM in contemporary parlance, which refers to science, technology, engineering, and mathematics), and a prolonged period of emerging adulthood that increasingly extends beyond adolescence (Arnett, 2002) are all plausible explanations for what seems to be a perceived increase in value for games as culturally respected artifacts.

Likewise, the rise of the gamer as an identity seems to indicate a status change, if not a fundamental argument in itself. Players have migrated toward player identities. Where once someone may have been an avid Space Invaders player, being a Minecraft (Duncan, 2011) player or firstperson shooter player comes with a community (Xu et al., 2011). Beyond the support of the Internet, player communities come with identities, affinity spaces, and privileges (Corneliussen, 2008). That these communities are privately managed, sometimes to the financial benefit of game-makers, is less important than the reality that such communities exist. The Game Developer's Conference, for example, which is the largest gathering of its kind, has supported a community management summit for years (http:// www.gdconf.com/conference/community.html), at which I have admittedly spoken.

Macro-persuasive procedural rhetoric is evidenced in the ways in which these groups aim to gain the cultural capital they seek. Most macro-persuasive play aims to mimic predecessors. The argument for games being an important and substantial industry once had a benchmark - more revenue than the movie industry (the reportedly outgoing dominant popular media 
industry). Nevertheless, once that benchmark was passed (Nath, 2016), games had to stand on their own and establish new standards of their own (Entertainment Software Association, 2015). The scale of the often-researched World of War Craft community, for example, offers the benefits of size and weight. At the game's popular height, these communities authored 297,496 pages of content (Wikia, 2017) and have been linked to positive social support (Longman et al., 2006) but have also shouldered the blame for a variety of game-related disasters, including child neglect.

Macro-persuasions not only come from federal, private, or public organizations; they also come from abhorrent, self-organized communities rallied around a few mantras. In the case of Gamer Gate (Parkin, 2014) and its widespread attacks, the macro-persuasion centered on some version of preserving games for gamers. The language of purity for games and real gamers sounds eerily similar to nativist and racist groups and is clearly anti-feminist (Chess \& Shaw, 2015). From the perspective of the supporters of Gamer Gate, games were a piece of their identity that needed to be protected, preserved, and defended from those who were seemingly attacking it (Salter, 2017).

Their strategies for winning this supposed culture war was a translation of that which they perceived from the outside as threatening. They produced pseudo-intellectual videos, outlining the errors in research produced by those they felt were attacking them, despite their own history of abuse (Salter, 2017). They also initiated investigations online, engaging in a somewhat television-like drama inspired by connecting disparate dots. At other times, their efforts wove intricate plots of espionage, funding, and conspiracy that were befitting of some of the most interesting games ever produced. They even rallied to participate in the academic processes of the organization at the center of much of their angst, the Digital Games Research Association (DiGRA). The community had planned to submit articles to the DiGRA annual conference, review papers, or even organize their own events.

The strategies for these individuals were primarily borrowed, i.e., they were strategies that bolstered the rhetoric of collusion in games or of an oppressed group of gamers who are having social justice games heft upon them unwillingly. In some ways, they followed Cialdini's weapons of influence (Cialdini, 1987), employing what was possible for a relatively unfunded group.

The persuasion comes from a few common mechanics for both ends of these persuasive game strategies. First, align games with something culturally valued. Second, mimic the characteristics of the signifiers for those culturally valued elements. Third, produce the evidence, through numbers and explanation, and then disseminate it to as wide an audience 
as possible, building a consensus first among those who already support your message.

These three steps can serve as a kind of playbook for effectively persuading society of the value of any enterprise. Although rather fundamental, this is a common strategy. It is, for example, why the logos and promotional elements of rising sports communities like World Quidditch and e-Sport's Major League Gaming look so eerily similar to Major League Baseball, the Olympics, or other well-regarded, culturally valued elements. True to the playbook, these formerly rising communities finish their rise with images and statistics to affirm their cultural value (i.e., 'If lots of people do it, it must be valuable'). This is the same logic that affirms the value of certain games conferences and e-sports.

If someone wants to employ macro-persuasions, the simplest strategy is to find an analogy to a respected cultural element, mimic that element, and then produce the evidence that there is a wide audience.

\section{The work of games, culture, and the rhetoric of production}

An important question to ask is whether games themselves have made the cultural rise or whether the labor in making them is really the element of rise. Unity, the makers of the software platform on which many game developers make their games, has seen far more financial success than the individual developers who subscribe to its services. The work of making games has continued to be attractive, despite the relatively low probability of it yielding either cultural or financial capital. Even for those games that are extremely valued, they rarely undergo the kinds of preservation efforts employed for other popular media (e.g., books and film). If the macro-persuasions have worked successfully and games have increased their cultural notoriety, why are there not more substantial efforts to preserve games?

While we argue that the work of making games is important, we rarely stop to ask about their preservation. This is in part because games have been, and continue to be, a disposable consumable, despite their rise toward the status of an artifact. We make games to feed our need to play. By analogy, the best games exist more like fine-dining experiences than high art. This is particularly evident in the ways we preserve games. To riff off of Bogost's keynote speech at the Games for Change Festival that talked about serious cheesecake, these games are treated like a decadent dessert we offer in two forms (Bogost, 2012): we either crystallize the game in its form, demonstrating it and isolating it from the larger space (like artificial food under glass and 
left in a window for display); or we offer it as a buffet to be consumed until players have gorged themselves until they are full. Game jams, for example, produce lots and lots of games, but their value is volume not quality.

Game jams are the bulk-value version of game manufacturing-lots of content with an emphasis on volume for cost purposes. Producing thousands of games in a single weekend offers the illusion of value. Admittedly, gamejam products are almost the inverse of bulk food. With bulk food, there is the occasional mistake. With game jams, there is the occasional success. This is, of course, completely fine, as the aim of a jam is not good product but good production. Jams are valued for their labor and their community, not for their process or the quality of their product.

The trick is that each of these efforts exists as a macro-persuasion. They each make distinct claims about games and their value to the society in which they exist. For several of these efforts that have blossomed in the last decade, there are simplified arguments to be witnessed in their practice. These are as follows:

\section{Game jams}

Game jams emphasize that their value is in the practice of making games. They often demonstrate how accessible game-making is and focus less on the final product. Much like the DIY community, the idea is not that each game is well-crafted but that it is self-crafted. Jammers argue that games can be personal, as an expression of the self, or that game-making is an achievement. They emphasize game value through origin over result.

\section{Games as craft}

The community of game-makers who champion games as craft is similar in spirit to game jamming but smaller in scale. These game-makers champion games as personal expression, as a medium for revolt, or as hand-hewn mass communication. This is most resonant in the work of Anna Anthropy and the workshops for Punk Arcade, but it is also expressed in commercial communities like Game Jolt or in tiny code contests.

\section{Games in galleries}

The value of games is, in part, that they are hand-hewn works in the material of the modern code. These coded artworks are worthy of sharing space with the historical work that we have all come to respect. 


\section{Games in culturally respected forms}

These offer ludic experiences or elements of the game community to make the claim that they belong there. These include the symphonic restyling of game soundtracks or theatrical performances informed by or based on games.

This list is not exhaustive but hints at the wider rhetorical efforts being made to legitimize digital play. These efforts each stand as a basis for their respective arguments about games but are neither unified nor concerted. There are, for example, few organizations that align all such macro-persuasions with a single mission. Instead, what is happening is that these efforts form a chorus that resounds with the heraldry of the value of games. If one feels the needs to defend game-playing or game-making, these provide the evidence that such work is not superfluous.

This raises an important question about the social context under which such a defense must be made: Why is it that so many people feel the need to defend games? Game-playing has existed for a long time, yet now the chorus and efforts to defend them continue to rise.

Is it perhaps that these macro-persuasions are the sociological precedent for legitimate cultural inclusion? Do all such popular efforts need their arguments to be made before they become accepted? And why, in a world where hyper-productivity is championed, do we feel the distinct and persistent need to defend play? Why must play be valuable if it is the opposite of the much-touted work? Why must play have purpose, and why must the organizations that support such play work to defend it? Lastly, why does it seem evident that we have been much more effective at making macro-persuasive arguments around the value of games and play than we have been at actually employing persuasive play?

\section{Games as industrial products}

On the other side of these efforts is the reality that the most substantial work in game-making has come from an industry that sees games very much as product, not artifact. They are more than a commodity because if that is all they are, they would lack the value that we seek to ascribe to them. This is, of course, the great tension between the academic games research community and some professional game-makers. Cultural artifacts are not commoditized. They are not counted in units sold; they are not localized, consumed, discarded, or recycled. In the Western view, culturally valued artifacts are not to be marketed, packaged, shipped, and sold. Yet games are 
all these things and more. For some, games are no different than consumer packaged goods, i.e., ideas come in at one end and then out come a pile of games.

Professional game-makers are not the only community to perceive games as such; there are consumers of games who view them in the same way. There are people seeking persuasive play who also see them like this. If the assumptions of an intellectual property focused on an ideology or technocracy are peeled from the polish of games, there are large communities who would argue that games are nothing more than products. In the most extreme scenario, games could be viewed as less than products and instead as byproducts. They are the byproducts of excesses of creative energy or the superfluous precipitate of idle time. These arguments are largely ignorant of the sociology and psychology of games, but they nevertheless persist.

In the end, it does not matter if the macro-persuasion is for or against games as an artifact or a product. The mere existence of the macro-persuasion indicates its value. Just as there may be games designed to argue for nonviolent resolution, there are also games that make the case for violence. Macro-persuasions for or against games as products or artifacts are merely evidence that the persuasions persist.

At times, the argument goes so far as to hoist a banner that reads 'games matter' in absolute defense of a medium and culture that seemingly argues against those who oppress it. In reality, games are no more oppressed than films and books, with the history of book-burning perhaps trumping the relatively limited censorship of games. Nevertheless, for game academics in particular, what is especially relevant is the need to make the macropersuasion in the pursuit of academic recognition, funding, and research support.

That games matter is baked into every political effort to do something more than just entertain with games. The macro-persuasion, then, is a cultural and political message that necessitates the construction of communities and institutions that support the value of play. These take the form of large-scale exhibitions, with aspirations to garner international respect and legitimacy and political organizations to support games. As with any large entity, the macro-persuasion is comprised of small micro-persuasive game efforts, including the individual game-makers and their efforts that populate these festivals, exhibits, and conferences.

Making macro-persuasions for the value of a product is much harder than doing so for the value of an artifact. So, we persist with games as artifacts. Yet of all the modern digital interactive media efforts, games have been the most successful in completing these efforts in macro-persuasion. Within the 
last ten years, games have completed a macro-persuasive cycle that affirms their cultural value, if not for the individual game then for the community of game-makers and the volume of games.

\section{Micro-persuasions}

On a smaller scale, there is the ever-growing collection of games that aim to persuade. These efforts include advergames, political games, games for change, advocacy games, editorial games, and others. In their sum, these games support the macro-persuasive rhetoric. Simply by their creation, they argue in support of the claim that games can have more than entertainment value. Their claims are evident in their own introductions and their own reasons for being. They argue that it is valuable to experience Native Alaskan culture (Never Alone) and that having a game about Fanta soda (Coca Cola, 2013), or associating the Burger King brand with a suite of mini-Xbox games persuades customers that the franchise is simultaneously hip and whimsical (King Games, 2006).

It is apt to call these games micro-persuasions, in part because most of them are noticeably smaller in their aspirations, budgets, and play length than their non-persuasive counterparts. For those who are unaware of the persuasive game domain, one of the first questions following any such presentation about them is: Why do these games not look like the ones on major consoles?

Of course, these games do not, in part, because the amount of effort and money committed to them is significantly smaller than their profit-making equivalents on game consoles and in stores. When a newspaper or private foundation invests in games, they lay down far less money than the world's leading game-makers.

It is these micro-persuasions that were at the heart of Bogost's original work and generally the notion most in the fore of many people's minds on the intersection of game design and persuasion. It is not uncommon to follow the basic line of thinking that lots of people play games and spend lots of time playing them. As a result, it would be great if someone could harness the power of such entertainment. This is the thinking that drives many into their first foray into micro-persuasive games. The arguments include meeting the audience where they are already engaged (Panic et al., 2013); creating more engaging experiences (Kors et al., 2015); telling better stories (Murray, 2017); or otherwise extending the power of games toward a specific purpose (Kahne et al., 2009). 
Micro-persuasions are the most documented and most apparent of the persuasive game design efforts. Readers are encouraged to review the published findings of others who have outlined the last ten years of persuasive play, specifically Grace (2012) and Humari et al. (2014). The prevalence of research into persuasive and purposeful play (Cater et al., 2014) is also noteworthy.

There are thousands of efforts to persuade through play. The literature demonstrating their success or failure is far less bountiful. In part, much like macro-persuasions, the value is in the making. Educators, for example, know that every first-year English major who is required to write a poem will not become Shakespeare. All the same, the work of trying to fit a verse into an iambic pentameter or to convey meaning in a sonnet is beneficial. It is the process that carries the education, not the final product or artifact. So, too, is the effort in persuasive play. While not every persuasive game is going to move the needle for efficacy, making them substantiates the third rule of macro-persuasions, namely proliferation.

As practitioners, there is a dilemma. It may be evident that practicing such design and implementation is productive, but so, too, is learning to draw, cook, or many other creative endeavors. The core questions should instead be: What is unique and productive when it comes to practicing making persuasive games? Does such work encourage critical thinking of system designs and meaning? Does it inspire more formal thinking about meaning in traditional entertainment? Answering yes to any of these questions engenders a sense of the value of such work and provides those who do it with a reason to continue doing so.

\section{Meta-persuasions, disinformation, and projected fiction as play}

Although much less of a distinct industry than, perhaps, its socio-technical and socio-political contemporaries, there are a variety of small games being played every day with a persuasive aim. These games include the 'fake news' profiteers, the internet trolls, and the champions of a variety of conspiracy theories. Of all the efforts in the persuasive games world, it is these individuals who have perhaps been most effective in terms of persuasive play. They have arguably elected national leaders, toppled viable candidates, and confused nearly every major population with arguments that, at times, even defy logic (Allcot, 2017). This is a combination of make believe, emulation, and fabrication.

This game is a game being played among the technological space that had been created to preserve thought (Leiner et al., 2009) and later evolved 
to become a force that affected democracy (Weare, 2002), sometimes negatively. With purveyors of fake news, for example, the internet functions as a playground in which not everyone understands the games that are being played or who is playing. Voluntary participation has persistently been essential to the definition of games. Games are played willingly, or they are not games. How, then, does fake news fit within such a paradigm? In part, it is because much of our unwitting support of fake news is contextualized within a game context. Fake news is shared via social media, which provides all the feedback systems of a conventional game. Players are encouraged to satisfy, to seek points in the form of likes, and to worry less about being right than being recognized. Fake news and rumor are nothing new, but the scale at which they can be disseminated has not only increased, the motivations for proliferating it have also expanded. Social media provides the ludic environment in which all players, whether producer or proliferator, are rewarded for their efforts. It is a successful gamification of sorts, but one less explicitly designed than resultant.

The results are clearly alarming. There are politicians who believe the number of supporters they have on social media but are unable to tell the difference between a bot and a personal account. There are full-time employees, working in conditions similar to the gold farms of the last decade (Heeks, 2009), who are seeking to produce profitable disinformation in the service of others (Bakir, 2017) or themselves (Sydell, 2016).

Each of these games is being played without the explicit consent of those being played with, but they are also lacking any referee or explicit mandate of opposition. As life goes, you can play a game and can be played with. As the ludic space of social media slips seamlessly into everyday experience, players seem to have lost their ability to identify when they are being played with. Social media images project a life that may not exist or a success when there is none (Hogan, 2010), which is another of the more mundane fake news efforts. Yet those who have engaged in projecting an idealized self on social media are, on a smaller scale, producing fake news. They, like the small armies of disinformation workers, are playing a game of lies with an audience that does not know it is being played with. How can someone who is playing their own game of a projected self be critical of better played and larger-scale games?

Meta-persuasive procedural rhetoric is evidenced in the ways in which these groups achieve their objectives. The meta-games are played with the same toys, blogs, websites, forums, and threads. These players operate within the elements of the game, but they employ them differently. They wreak the most havoc not when they make their own games but when they operate within contexts 
differently. By playing games within games, they wreck half-constructed forms of gamification. By analogy, they are like children playing tag in the middle of a hopscotch game. The disruption is apparent, but the reason is not. The Internet's fluidity and anonymity only expand the shadow of such play, making it seem larger and grander than it is. Yet these players are not master designers or players. They may have played tag through your hopscotch, but now they are playing cards in the middle of someone else's shuffleboard.

One admitted fundamental weakness in understanding meta-persuasive play in this frame is the tension of employed players. As we have come to accept play as voluntary from at least the time of Huizinga forward, the ambiguity of professional play persists. Purveyors of fake news, for example, do not typically view their work as consequential (Sydell, 2016). The fictions they created are just that-fictions. Profitable fictions exist in the way scriptwriters profit from their fiction and animators present worlds that do not really exist. By this framing, fake news production is not a diabolic effort to dismantle democracy; it is a playful way to pay the bills.

Historically, such play has a precedent in the creative play of Big Games (Lantz, 2006) and in the art work of everyone from Situationists (Debord, 1957) to ludic happenings in the 1960 (Berghaus, 1993). The disquieting truth is that fake news bloggers have created more of a stir on their laptops than artists in the 196os ever did on the street. The tools just seem to be more effective now.

More importantly, what these efforts demonstrate is a tendency toward persuasive play in non-game contexts. The games these individuals play have plagued and complicated the daily operations of legitimate news organizations (Waldrop, 2017). It is this efficacy, however detrimental, that demonstrates that persuasive play can work. As case studies, they provide evidence that such play works best when it employs the normal elements of operation-in short, playing within the rules of play but subverting them. In the vernacular, they game the system, and in so doing demonstrate a different way to play. From this perspective, they are similar to micro-persuasion efforts like Critical Gameplay (CriticalGameplay.com) and the work of Molleindustria (MolleIndustria.org). The main difference, as mentioned, is that the games of fake news and social media projections are not always apparent to those who are watching them being played.

\section{Conclusion}

Returning to the wider questions contained in this chapter's introduction, the why for persuasive play becomes apparent. Why do researchers and 
practitioners want to persuade people through games? This is probably because games permeate society at multiple levels, which is demonstrated in everything from political discourse to information manipulation and to the allocation of resources in the pursuit of knowledge. Games work at the macro-level of large-scale systems and institutions, the micro-level of individual play experiences, and the meta-levels between them. Admittedly, there are byproducts at every level that do not always work toward pro-social ends.

Why are games the right medium for delivering persuasive messages? It is likely that they are not the right or wrong medium, because they are less of a medium than a practice with which contemporary society continues to re-engage. Games are played, practiced, tweaked, and redesigned as part of a continued effort to attempt to explain and understand. Adopting the view of games as a medium focuses on the product or artifact of games, which ignores the greater part of persuasive play. Doing so obscures the macro-persuasions, the games within society that propel the medium to something more than a medium. It also misses the meta-persuasions, which employ ludic solutions to achieve their purpose. Whether that purpose is getting more 'likes' on social media or affecting political views, its results are shown in changes in interests, activities, and opinions. The preponderance of selfies with the aim of achieving fame on social media, for example, clearly demonstrates an activity change that outshines any such efforts of a micro-persuasive game. The national entrenchment or nativist sentiments occurring across the world could also likely be the product of a series of opinion-altering meta-persuasions.

Why has the public discourse come to need games as a vehicle for communicating and argumentation? Years ago, the obvious claim was the opportunity for games to explain complex systems, to afford simulation for deep investigations of inquiry-based learning, or simply to meet the experiential demands of changing demographics. Yet the reality is that the depth of engagement is losing out to the shallow experience of social media headlines and easy-to-share infographics. Instead, what persuasive play at all levels demonstrates is that humans operate in game contexts far more often than they may realize. The need for games is not new, but it is more easily identified because of this research into games. Psychology or cell biology have always existed, but our understanding and analysis of them have only occurred relatively recently in human history.

It is, then, more likely that there is no need to gamify because games have always existed. Therefore, persuasive play is less about finding ways to create new persuasive games but is instead a case of appropriately examining, 
identifying, and playing the games that already exist. This is the secret to the success of the great fake news authors and the projectors of social media myths.

Perhaps what is most important is not demonstrating that persuasive play can or cannot work. Instead, we may need to examine or accept the desire to make it work. Perhaps we are seeking something to fill a greater hole? More likely, the persistence of these macro, micro, and meta-persuasions signals an evolution, a revolution, or an apocalypse of media consumption and production habits. Perhaps in another 25 years we will find that we are incapable of understanding an argument without the machinations of play or that arguments presented without play are too simple and antiquated. Much like the death of classical oration, conventional argumentation without the interactive depth of play may seem archaic, clunky, and lacking contemporary nuance. A simple argument may become just that- too simple to be taken seriously.

\section{References}

Allcott, H. \& Gentzkow, M. (2017). Social Media and Fake News in the 2016 Election. Journal of Economic Perspectives, 31(2), 211-236. doi:10.3386/w23089.

Arnett, J.J. (2002). The Psychology of Globalization. American Psychologist, $57(10)$, 774. doi: 10.1037/0003-066X.57.10.774.

Bakir, V. \& McStay, A. (2017). Fake News and The Economy of Emotions: Problems, Causes, Solutions. Digital Journalism, 6(2), 154-175. https://doi.org/10.1080/2167 0811.2017.1345645.

Berghaus, G. (1993). Happenings in Europe in the '6os: Trends, events, and leading figures. TDR (1988-), 37(4), 157-168. doi: 10.2307/1146300.

Bogost, I. (2007). Persuasive Games: The Expressive Power of Videogames. Cambridge, MA: MIT Press.

—. (2012). G4C13 Keynote. Youtube. Retrieved 2 July 2018 from https://www. youtube.com/watch?v=GBduFJUdoog.

Boyle, E.A., Hainey, T., Connolly, T.M., Gray, G., Earp, J., Ott, M., Lim, T., Ninaus, M., Ribeiro, C., \& Pereira, J. (2016). An Update to the Systematic Literature Review of Empirical Evidence of the Impacts and Outcomes of Computer Games and Serious Games. Computers \& Education, 94 (March 2016), 178-192. https://doi. org/10.1016/j.compedu.2015.11.003.

Carter, M., Downs, J., Nansen, B., Harrop, M., \& Gibbs, M. (2014). Paradigms of Games Research in HCI: A Review of 10 Years of Research at CHI. In Proceedings of the First ACM SIGCHI Annual Symposium on Computer-Human Interaction in Play, 27-36. New York, NY: ACM Press. https://doi.org/10.1145/2658537.2658708. 
Chess, S., \& Shaw, A. (2015). A Conspiracy of Fishes, or, How We Learned to Stop Worrying About\# Gamergate and Embrace Hegemonic Masculinity.Journal of Broadcasting \& Electronic Media, 59(1), 208-220. https://doi.org/10.1080/08838 151.2014.999917.

Cialdini, R.B. (1987). Influence: The Psychology of Persuasion. New York, NY: Collins Business Essentials.

Coca Cola (2013). Fanta Fruit Slam 2. [Android Game].

Corneliussen, H. \& Rettberg, J.W. (eds.). (2008). Digital Culture, Play, and Identity: A World of Warcraft reader. Cambridge, MA: MIT Press.

De Lange, M. (2015). The Playful City: Using Play and Games to Foster Citizen Participation. In A. Skaržauskienè (ed.), Social Technologies and Collective Intelligence, pp. 426-434. Kaunas, Lithuania: Mykolas Romeris University.

Debord, G. (1957). The Situationists and the New Forms of Action in Politics and Art. On the Passage of a Few People Through a Rather Brief Moment in Time. In McDonough (ed.), The Situationist International. Cambridge, MA: MIT Press.

Demby, E. (2011). Psychographics and From Whence It Came. Decatur, Georgia: Marketing Classics Press.

Duncan, S.C. (2011). Minecraft, Beyond Construction and Survival. Well Played: A Journal on Video Games, Value and Meaning, 1(1), 1-22. https://doi.org/10.1184/ R1/10029221.v1.

Entertainment Software Association (2016). Sales, Demographic, and Usage Data: Essential Facts About the Computer and Video Game Industry. $E S A$. Retrieved 25 July 2018 from https://techraptor.net/gaming/news/ esa-releases-2016-essential-facts-report.

Gee, J.P. (2004). Learning by Design: Games as Learning Machines. E-Learning and Digital Media, 2(1), 5-16. https://doi.org/10.2304/elea.2005.2.1.5.

Grace, L. (2012). A Topographical Study of Persuasive Play in Digital Games. In Proceedings of the Academic Mindtrek (Tampere, Finland, 3-5 October 2012). New York, NY: ACM Press.

Grace, L.D. (2017). Heuristics from Curating and Exhibiting Game Art in the 21st Century. In ARTECH2017: Proceedings of the 8th International Conference on Digital Arts. https://doi.org/10.1145/3106548.3106607.

-., \& Farley, M. (2016). How Game Design Thinking Becomes Engagement Design. In Proceedings of the 2oth International Academic Mindtrek Conference, 281-286. New York, NY: ACM Press.

Greenfield, P.M. (1994). Video Games as Cultural Artifacts. Journal of applied developmental psychology, 15(1), 3-12. https://doi.org/10.1016/0193-3973(94)90003-5. Hamari, J., Koivisto, J., \& Pakkanen, T. (2014). Do Persuasive Technologies Persuade? A Review of Empirical Studies. In International Conference on Persuasive Technology, 118-136. Cham, Switzerland: Springer International Publishing AG. 
Heeks, R. (2009). Understanding "Gold Farming” and Real-Money Trading as The Intersection of Real and Virtual Economies. Journal for Virtual Worlds Research, 2(4). https://doi.org/10.4101/jvwr.v2i4.868.

Hogan, B. (2010). The Presentation of Self in the Age of Social Media: Distinguishing Performances and Exhibitions Online. Bulletin of Science, Technology \& Society, 3o(6), 377-386. https://doi.org/10.1177/0270467610385893.

Jenkins, H. (2005). Games, the New Lively Art. In Raessens, J. \& J. Goldstein (eds.), Handbook of Computer Game Studies, 175-189. Cambridge, MA: MIT Press.

Kahne, J., Middaugh, E., \& Evans, C. (2009). The Civic Potential of Video Games. Cambridge, MA: MIT Press.

King Games (2006). Sneak King [Xbox Game].

King, B., Borland, J., \& Stewart, R. (2003). Dungeons and Dreamers: The Rise of Computer Game Culture from Geek to Chic. New York, NY: McGraw-Hill/ Osborne. Kors, M., Van der Spek, E.D., \& Schouten, B.A. (2015). A Foundation for the Persuasive Gameplay Experience. In Proceedings of the Foundations of Digital Games. Retrieved 12 May 2018 from http://www.fdg2015.org/papers/fdg2015_paper_28. pdf.

Lantz, F. (2006). Big Games and the Porous Border Between the Real and the Mediated. Vodafone Receiver Magazine, 16. Retrieved 1 February 2017 from: http:// www.receiver.vodafone.com/16/articles/indexo7.html.

Leiner, B.M., Cerf, V.G., Clark, D.D., Kahn, R.E., Kleinrock, L., Lynch, D.C., Postel, J, Roberts, L.G., \& Wolff, S. (2009). A BriefHistory of the Internet. ACM SIGCOMM Computer Communication Review, 39(5), 22-31. http://doi.acm.org/10.1145/1629607.1629613.

Murray, J.H. (2017). Hamlet on the Holodeck: The Future of Narrative in Cyberspace. Cambridge, MA: MIT press.

Nath, T. (2016). Investing in Video Games: This Industry Pulls in More Revenue Than Movies, Music. Nasdaq. Retrieved 3 April 2019 from: http://www.nasdaq. com/article/investing-in-video-games-this-industry-pulls-in-more-revenuethan-movies-music-cm634585.

E-Line Media (2014). Never Alone [Digital Game].

Panic, K., Cauberghe, V., \& De Pelsmacker, P. (2013). Comparing TV Ads and Advergames Targeting Children: The Impact of Persuasion Knowledge on Behavioral Responses. Journal of Advertising, 42(2-3), 264-273. https://doi.org/10.1080/oo9 13367.2013.774605.

Parkin, S. (2014). Gamergate: A Scandal Erupts in the Video-Game Community. The New Yorker, 17. Retrieved 3 March 2018 from https://www.newyorker.com/tech/ annals-of-technology/gamergate-scandal-erupts-video-game-community.

Plummer, J.T. (1974). The Concept and Application of Lifestyle Segmentation. The Journal of Marketing, 38(1), 33-37. doi: 10.2307/1250164. 
Salter, M. (2017). From Geek Masculinity to Gamergate: The Technological Rationality of Online Abuse. Crime, Media, Culture, 14(2), 247-264. https://doi. org/10.1177/1741659017690893.

Squire, K. (2011). Video Games and Learning: Teaching and Participatory Culture in the Digital Age. New York, NY: Teachers College Press.

Sydell, L. (2016). We Tracked Down a Fake-News Creator in the Suburbs. Here's What We Learned. All Tech Considered. Retrieved 7 April 2018 from https://www.npr.org/sections/alltechconsidered/2016/11/23/503146770/ npr-finds-the-head-of-a-covert-fake-news-operation-in-the-suburbs.

Waldrop, M.M. (2017). News Feature: The Genuine Problem of Fake News. Proceedings of the National Academy of Sciences, 114(48), 12631-12634. https://doi. org/10.1073/pnas.1719005114.

Weare, C. (2002). The Internet and Democracy: The Causal Links Between Technology and Politics. International Journal of Public Administration, 25(5), 659-691. https://doi.org/10.1081/PAD-120003294.

Wikia (2017). World of Warcraft. Retrieved 8 March 2018 from http://wowwiki. wikia.com/wiki/Portal:Main, last accessed on 14/12/2018.

Xu, Y., Cao, X., Sellen, A., Herbrich, R., \& Graepel, T. (2011,). Sociable Killers: Understanding Social Relationships in an Online First-Person Shooter Game. In Proceedings of the ACM 2011 conference on Computer supported cooperative work, 197-206. New York, NY: ACM Press. https://doi.org/10.1081/PAD-120003294.

\section{About the author}

Lindsay Grace is the Knight Chair of Interactive Media at the University of Miami. He was founding director of the American University Game Lab and Studio where he piloted more half a million dollars in persuasive game design in three years. His game designs have received awards from the Games for Change Festival, Meaningful Play, and others. 



\title{
8. VilDu?! A Game for Sexually Abused Children: How Openness Facilitated a Clear Design Direction
}

\author{
Menno Deen' \& Eline Muijres
}

\begin{abstract}
This chapter narrates the design process of VilDu?!, a game, or therapeutic tool, for sexually abused children. The game was developed during the Lyst Summit game jam and proved an important milestone for the Games [4Therapy] Project. Lessons learned from the design experience and the game's implementation process in therapy sessions have contributed to a design approach for therapeutic games. This article will elaborate upon this by chronologically describing the initial challenges of the project, the design process and insights gained from developing VilDu?!, and how the game is now used in therapy sessions.
\end{abstract}

Keywords: games for therapy; games for change; research-through-design; game jams

\section{Introduction}

This chapter discusses the design process for VilDu?!, a game, or therapeutic tool, for sexually abused children. The game was developed during the Lyst Summit game jam and proved to be an important milestone for the Games [4Therapy] Project. Lessons learned from the design experience and the game's implementation process in therapy sessions have contributed to a design approach for therapeutic games.

1 This chapter has been written in first person by Menno Deen with the collaboration of Eline Muijres.

Hera, T. Dela, J. Jansz, J. Raessens, B. Schouten, Persuasive Gaming in Context. Amsterdam: Amsterdam University Press, 2021 DOI 10.5117/9789463728805_CHO8 
This chapter will elaborate on this by describing chronologically the initial challenges of the project, the design process, insights gained from developing VilDu?!, and how the game is now used in therapy sessions. It will become clear that three affordances (Norman, 1990) of games facilitate conversations and lower boundaries to talking about sexual abuse: focus, non-normativity, and ambiguity (Sutton-Smith, 1997). We will revisit these after discussing the design process.

\section{Background: Why make a game for sexually abused children?}

In 2014, Fontys University of Applied Sciences commenced a project called Games [4Therapy]. Its purpose was to study how games could enrich therapy practices and how they could be implemented. Its target audience was primarily teenage boys who have difficulty externalizing problems. In short, this means that, according to these teenagers, the problems and difficulties they face originate predominantly outside their sphere of influence. In layman's terms, 'it's always someone else's fault'. Problems are therefore 'externalized', resulting in behavior for which teenagers rarely take responsibility.

Teenagers who externalize problems are often treated for multiple psychological disorders. This comorbidity frustrates the design and validation of new interventions. As every case is unique and context-dependent, depicting a clear design goal is a challenge. What is more, the integration of new interventions - in this case games-into the overall structure of therapy sessions creates other difficulties. One of these concerns my inability to identify with clients: I did not understand either their perspective or their problems. Combined with the suggested comorbidity of psychological disorders, I failed to identify a core denominator to design for.

In order to remedy the situation, I decided that adhering to a researchthrough-design approach (Laurel, 2004) would help me to understand clients better and enable me to produce some games in the process. We therefore created student projects around the theme and organized various game jams. These fast-paced design sessions, where participants design a playable prototype within a specific time limit (Deen et al., 2014), appeared to be an ideal method for exploring the design process of therapeutic games. It appeared that other designers had the same problem: it was hard to identify the core denominator of a mental challenge, and so it was hard to design a game that targeted a particular aspect of it.

Among other events, we organized a Games [4Health] Jam on Mental Health (2015) and Games [4Research] Jams (2013-14) at two Computer Human 
Interaction conferences. These jams attracted designers and developers from the Netherlands and abroad, with a rich variety of games produced in just one weekend. However, none of the therapists seemed to be particularly interested in any of the prototypes developed. It was suggested by some of them that you could not expect game designers to fully understand the merits of a psychological challenge in a mere weekend, as these problems are multifaceted, complex, and intertwined. Designers were assumed to not have a full understanding of this and, therefore, it was believed that they could not design a useful game for therapy purposes.

However, in defense of the designers, it was somewhat difficult for the therapists to transfer their knowledge on the subject matter, nor did they seem able to present the designers with a clear design direction. Most of the therapists appeared to be reluctant to choose just one 'core' denominator for a mental challenge; they suggested that the comorbidity of mental illnesses created a certain complexity that made every case a unique problem that could not be 'fixed' with a single game. Additionally, the therapists often suggested that they were not in a position to design intervision, as they considered themselves to be unqualified in this respect. Most of them held a BA in psychology, while the design and validation of therapeutic interventions was deemed to 'clearly be the job' of someone holding a Master's.

Finally, the process was limited by the contrasting cultures of mental health professionals and game designers. Unaccustomed to the hierarchical relations and implicit politics in mental health institutions, I found it hard to communicate with therapists and managers. Finding the right tone of voice was very difficult. My interviews and questioning techniques were often considered to be aggressive and condescending, which did not help in our communications. Finishing my PhD appeared to soften this socio-cultural issue of trust and hierarchy somewhat, but the damage had already been done.

Frustrated with the lack of progress, I decided to create a situation that would remedy the challenges set out above. I found this in the game jam at the Lyst Summit (Jarnfelt \& Hasselager, 2014), which dealt with sex, love, and romance in games. Jarnfeldt and Hasselager invited me to speak on behalf of Games [4Diversity], and I presented a talk about our latest jam session on LGBTQ+ perspectives. Then, after the presentations, everyone took part in the Lyst game jam.

The Lyst game jam provided the perfect opportunity to remedy the challenges referred to above. I figured that bringing close friends to the jam would help me to overcome cultural differences and hierarchical politics. I also thought that working on a different mental challenge (other than the 
one defined in our project) would help me to define a core design direction that could be transferred to our project. I decided to create a therapeutic game for sexually abused children. This was within the theme of the Lyst Summit but, more importantly, my friends had already 'worked' on this kind of problem. Lastly, I believed I could make a positive contribution to an issue that was rather personal to me or, more precisely, to a friend.

I invited my close friend, Marcel (a psuedonym), to accompany me. He had been sexually abused in the past. We have known each other for a long time and have talked about his experiences at length. I believed his presence would enable me to understand the complexity and comorbidity of his problems. I also brought Frank Lips with me; he is another friend and therapist with extensive knowledge of therapy for families where intimate boundaries have been transgressed. As Lips and I are also good friends, there was no hierarchy to speak of. Furthermore, since all the people joined in the process of their own volition, and with a shared goal—-designing something that could help a victim of sexual abuse - there were no politics involved.

The goal set out above was shared with two developers, Tim Pelgrim and Paul Bierhaus. Having known both of these people during my time at Ranj Serious Games, I was certain I could rely on them to be open-minded and, more importantly, non-normative about divergent relationships. The non-normativity and respectful manner in which they treated both the subject and Marcel proved to be an important asset in the design process.

By focusing on a completely different mental challenge, by working with friends, and by traveling to Copenhagen to enroll in a game jam, I could literally distance myself from a rigid way of working. We felt safe and secure during the jam and, thanks to this experience, were able to open up to one another. Although the beers helped, I suspect that the privacy offered by our language (speaking Dutch in Denmark) and the inclusively designed Lyst Summit organized by Jarnfeldt and Harselager were more important when it came to our ability to get to the heart of the problem.

The open atmosphere in our group, the short duration of the jam sessions, and the privacy offered by our language presented us with a core denominator to design for. This core issue does not cover the full extent of the problems that sexually abused children face. Instead, it just offered us a clear design goal to develop a tool that could contribute (in a small way) to the healing process of such a traumatic experience.

In retrospect, the design-through-research approach gave us various insights into a possible strategy for designing therapeutic games. For one, it became clear that it was best for designers (and therapists) to avoid overcomplicating a mental problem; instead, it appeared to be helpful to 
'subtilize' the issue. I will explain this later, as well as the statement 'design for ambiguity', with both propositions building on one main value that the design team tried to uphold, namely designing a non-normative space.

It appears that if you want to design a space where clients feel comfortable enough to talk about difficult issues, then the design process itself should also be characterized as such.

\section{Method}

The research method on therapeutic games has strong connections to research-through-design approaches, where researchers work with domain experts, end-users, developers, and designers to co-create a prototype (Laurel, 2004). By producing a phenomenological narration of the design process, we can reflect on design decisions and suggest possible strategies for designing therapeutic games. This approach has an advantage over more theoretical and literature-based methods: it illuminates practical implementation issues that can only be identified in an actual design experience.

We organized various game jams to gain a quick insight into the practical implications of designing therapeutic games. Lessons learned from the Games [4Research] Jams held in CHI 2013 and CHI 2014 suggest that game jams can used for academic research. In the ACE conference paper, Deen, Nack, and Haggis (2015) suggest that a jam helps to narrow down the research question to a useable statement; create a clear focus and understanding of the practical implementations; share knowledge and inspire; iterate rapidly; create a safe environment for failures; and work outside one's comfort zone. Lastly, organizing a game jam as a conference workshop brings together different perspectives and enables easy access to play-testers, expert reviews, and peer discussions.

Reflecting on this design process and the implementation of the game in therapy sessions creates an insight into the value of our design approach. One limitation of this approach is that these insights are rather biased and prone to the subjective position of the designer. In order to stipulate that this chapter discusses anything but a personal design experience, I decided to write from a personal perspective. The use of the first person in this chapter refers to me, Dr. Menno van Pelt-Deen, highlighting my personal involvement and viewpoint in this reflection.

The next sections describe:

1. the problems we faced during the initial design process

2. how we found a clear design direction 
3. the first game

4. expert reviews and recognition

5. further development and iterations

6. the implementation of the game in therapy sessions

7. lessons learned from the design process.

The chapter concludes with recommendations for future research as well as a design strategy for therapeutic games that may facilitate conversations about tough subjects.

\section{The initial design process}

In April 2014, I traveled to Copenhagen together with Lips, Pelgrim, Bierhaus, and Marcel to participate in the Lyst game jam. After a full day's conference, the jam started around five o'clock in the evening, when individuals were grouped together and the first concept-development sessions started. Typically, these sessions are relatively short, normally taking one or two hours. The concept phase is brief by design in order to offer enough development time. This was not, however, the case for VilDu!?.

The design process of VilDu?! involved a great deal of preparation, discussion, and alcohol. When the group started to design, it immediately split into two, very distinct camps. We all wanted to create a game that was fun or, more precisely, comical and light-hearted. The designers wanted to produce a game that explored intimate boundaries in a safe way, while the other camp appeared to be more focused on teaching children to recognize unwanted intimate activities and educate them in boundary setting.

After four hours of futile brainstorming, play-testing, and heated discussions, Marcel stood up. He suggested narrating his experience from beginning to end. Everyone agreed, and so Marcel talked for two hours straight, spelling out his anger and every insecurity as well as joyful aspect of the abuse he experienced more than eighteen years ago.

Telling the full story took a significant toll on Marcel. He needed to open up, relive terrifying moments, fight feelings of guilt and shame, and put his story in an understandable, chronological order. He was clearly fatigued after this ordeal, but his effort did hit a mark: all the team members seemed to understand what it meant when boundaries were transgressed on such an intimate level.

Minutes after Marcel talked, one of the other team members spoke up, sharing a story in which his intimate boundaries were also transgressed. It 
did not take long before everyone in the group shared a similar experience. Indeed, it transpired that everyone on the team had had his/her boundaries transgressed in one way or another. And although their experiences did not involve a huge age difference, tapping into less traumatic events helped us to identify a core problem and thus a clear design direction.

While opening up to one another, we identified two commonalities. First, it appeared that setting boundaries was extraordinarily hard. None of us wanted to say 'no' during sex for one reason or another. And second, it proved to be difficult to find the right 'tone of voice'. Team members struggled with describing body parts and sexual activities that fitted the tone of the discussion. Sex appeared to be ambiguous in essence (exciting and relaxing, crossing and respecting boundaries at the same time). Our vocabulary thus fell short when it came to accurately describing these multiple interpretations of an activity.

We set ourselves the goal of helping children to say no (or at least getting across to the children that it was okay to say no), and we wanted to give them a non-verbal way to communicate. We therefore set out to create a game that helped them to speak without the need to talk.

\section{A clear direction and problem space}

It appeared that the team had found a clear direction and problem space. A literature study substantiated our assumptions. It appears that less than 10 percent of sexual violence is reported. This creates a problem for a significant part of our community, since more than a third of women and more than a tenth of men are subjected to sexual violence (van Berlo \& van Beek, 2015; Yancey, Hansen, \& Naufel, 2011) Even though many people have such experiences, they rarely talk about it or it takes a long time for victims to dare to speak up.

Yancey, Hansen, and Naufel suggest that the main inhibitors of disclosure are related to fear, shame, and self-blame. Additionally, the lack of language is a common barrier among sexually abused children (Hunter, 2010). It is hard to talk about abuse, which may hinder the development of coping strategies and, as a result, the healing process after the traumatic experience. This is why therapists try to create an atmosphere that conforms to the victim's preferred way of communication. This communicative practice should be safe and fitting for the child, and one of these practices is play.

Play provides children with the live, dynamic, and individual language that is indispensable for the expression of their subjective feelings and for 
which collective language alone is inadequate (Piaget, 1964). This is one of the reasons why play therapy can help victims of sexual abuse; play can lower barriers to describing experiences in non-verbal ways. This is especially true for non-directive child-centered play therapy (Hill, 2009; McMahon, 1992), where children are in control within some limits that are gently but firmly set. Most playful interventions in therapy sessions are analogue systems: they use pen and paper, physical role-play props, and sometimes a digital text processor. There are, though, surprisingly few digital interventions that facilitate conversations about sexual abuse.

I was particularly surprised by this because I consider digital interventions to be a rather strong medium for empowering people (Deen, 2015). Video games are particularly well equipped to create an autonomous, supportive learning environment that offers players the opportunity to explore, struggle, and then explore some more (Deen \& Schouten, 2014). Most digital interventions can, however, be characterized by their restrictive gameplay and one-directional communicative practices. They are not autonomy-supportive in any way and seldom empower children to develop their own values and norms when it comes to sex, love, and intimacy. Instead, they are restrictive, obstruct the actual content with metaphors, and/or are rather normative in that they suggest what is right and wrong without enabling children to depict that for themselves.

\section{Restrictive games}

The interactive movies of Can You Fix It? (IJsfontein, 2013a) portray various youngsters in their search for intimacy. The series covers all kinds of sexual boundary transgressions but lacks pedophilic relationships. Additionally, it does not directly invite players to talk about their experiences, although this nonetheless happens. Can You Fix It? is used in classroom settings and has some viral qualities. According to the developer, the episodes that situate naked breasts and a gay romance are viewed the most. This happened without any marketing effort, suggesting that interest in the game grew by word of mouth.

Another Dutch-language app by IJsfontein is called Beter in Bed (2013b) (which translates into 'perform better in the bedroom'). This contains a 'duo-test' in which players are prompted with questions about sex and intimacy. If their answers correspond, players have a 'match'. This duo-test resembles Game Oven's Friendstrap (2013), a multi-player game for the phone in which players are prompted with awkward conversation subjects. Some of the topics have a sexual connotation but do not necessarily offer players the 
means to explore intimacy in a different way; the game just offers prompts to talk about but does not change the communicative practice itself.

We wanted to create a game that changed the communicative practice, e.g., offering players the ability to create their own language, building on knowledge from open-ended play (T. Bekker \& Sturm, 2009; T. Bekker, Hopma, \& Sturm, 2010), where players can create their own rules and games. In other words, we wanted to create a playful environment that was less restrictive than the linearity of Can You Fix It? but offered more direction than Friendstrap.

\section{Metaphoric games}

The applications described above mainly target adolescents and adults. As such, they may not resonate well with children's beliefs and experiences. What is more, they are rather text-oriented, using culturally depicted phrases and words. We thought that this might be problematic because children often lack the vocabulary to talk about sex, love, and romance.

Some developers appear to remedy this vocabulary challenge by enabling communication through metaphors. For example, the Child Safety Research \& Innovation Center developed Sydney Safe-Seeker and the Incredible Journey Home (2008) to educate children to recognize sexual predators. Quite literally, the sexual predators in Sydney Safe-Seeker graphically represent animals that are known predators. Although metaphors are commonly used in psychotherapy to frame issues, we felt that the anthropomorphic characters of Sydney Safe-Seeker were a bridge too far, especially because they were so culturally (read: American) depicted in order to connect to non-Western children.

Additionally, we wondered if it was the child or the therapist who was actually afraid of being explicit. Lips assured us that therapists also experience problems finding the right words in therapy sessions. However, in his opinion, this is an important part of the healing process. Finding the terminology for body parts and sexual acts and doing so together with one's therapist is an integral part of the patient's process of coming to terms with the traumatic experience.

We decided that the game should be rather explicit and should not shy away from using words or imagery that could be considered offensive (to parents, therapists, or other grown-ups). It became an obvious design statement that we wanted the game to be clear about sex and offer children the opportunity to interact with a penis, vagina, tongue, and other body parts without being 'uptight' about this. 


\section{Normative games}

We identified less metaphoric prompts and thus more inspiration in the game Orbit (University of the Sunshine Coast, 2012). This game allows players to assist the crew of an orbiting station while at the same time being prompted with multiple-choice questions about intimacy. Although the game feels less metaphoric and may therefore be easier for a player to connect to his/ her personal circumstances, it still dictates how children should behave in particular scenarios. Some behavior is considered to be 'not right' or simply 'wrong'. These normative statements problematize an already difficult situation; instead of asking children how they value intimacy, the game dictates the norm.

This normativity is present in Can You Fix It? as well. Players watch the unfolding of a story about an intimate boundary transgression. At predefined points, players can hit the 'fix it' button and decide how they would like the story to continue. Will the boy ask what she means by 'top or bottom' or will he 'graciously' ignore her statement on a first date? Although players can decide what and when to interact, the stories and their premises are predefined by the maker of the website, the governmental health agency Sense. Although the movies often result in valuable debates, the website itself is normative.

The same is true for the duo game in the Beter in Bed app. While players are able to decide if they like a particular sexual activity, the app suggests that you only 'score' when both partners want the same thing. This is a less obvious normative statement, as it feels more like a game mechanic, but it still suggests that both individuals should enjoy the same things in a healthy relationship. This does not, however, hold true for many relationships and sexual endeavors.

We wanted as few normative statements as possible in our game. Again, taking inspiration from open-ended play, we figured that the game should feel like a 'sandbox game' in which players can create their own stories. A good example of a game that steers direction but does not necessarily depict strong norms and values is the Toca Life series, which offers children a digital dollhouse/sandbox within a specific theme.

In Toca Life: Hospital (Toca Boca, 2017), players can interact with various characters and things found in a generic hospital. The game does not shy away from portraying patients with broken limbs or undergoing radiography and organ donations. However, the game omits any textual markers: it is up the children to create their own story. I observed my niece playing the game. She was loudly talking to herself, creating one story after another while negotiating with the game. A similar interaction is found in the end 
result of VilDu?!, where we carefully construct an autonomy-supportive environment in which we tried to be as non-normative as possible.

\section{Our design rules: open, explicit, and non-normative}

In summary, we wanted our game to be less normative and less restricted in order to facilitate a conversation in a safe and open space.

With our game, we wanted to give children a voice without the need to talk. We wanted to put children in charge of their story, normative structure, and values associated with love, sex, and romance.

In order to understand how to present children with a voice, we studied existing ways in which people talk about sexually intimate acts in a less textual manner. We found inspiration in online sexting and pornographic play environments.

\section{Sexting, cybersex, and role-play environments}

Particular patches and worlds in Second Life (Linden Lab, 2003) have players explore their sexual fantasies in many ways. Explorations in gore, BDSM, and gay role-play appeared to be rather active in these virtual environments. It appeared that the virtual doll-play-like interactions lowered the boundaries for exploring intimate fantasies. In addition, it may be that the anonymity of these environments empowers users to explore less socio-culturally accepted fantasies.

Other examples were IMVU (IMVU Inc., 2009), Snapchat (sexting) (Spiegel, Murphy, \& Spiegel, 2011), World of Warcraft (sex in, for example, the GoldShire Inn; Blizzard Entertainment, 2004), ChatRoulette (Ternovskiy, 2009), and Habbo Hotel (Karjalainen \& Kyrölä, 200o). All these examples leverage the relative anonymity and connectedness of the internet (ChatRoulette, Snapchat) and use digital doll play to suggest sexual activity (IMVU, World of Warcraft, and Habbo Hotel). We were surprised that none of the digital environments had originally set out to do this but that particular aspects of the virtual space facilitated play.

\section{Emergent games}

Emergence occurs when more or less simple rules interact to give rise to behavior that was not specifically intended by the developer of a system (Kickmeier-Rust 
\& Albert, 2009). This is what Salen and Zimmerman (2003a, 2003b) have dubbed emergent gameplay. To some designers, and especially to me, this is the greatest value of games, namely offering players the opportunity to create their own play.

If we look at games that create rich emergent gameplay, the Toca Life series discussed above comes to mind. While creating one's own play, new stories and play-forms emerge. Moving a character forward on a horse in Toca Life Stable could mean galloping freely through nature or performing in a highly contested championship. The player decides what the interaction means, not the designer.

In the example above, interactions could mean two or more things at the same time. This is what Sutton-Smith (1997) calls the design for ambiguity. Sutton-Smith states that playful interactions can have various, sometimes dichotomous meanings. The example of giving one's own meaning to riding a horse is a pivotal value of play and emergence. In Toca Life, the series are culturally depicted and steered by the context designed by the game. Children then draw their own conclusions by interacting with the game.

In other words, children draw normative conclusions about the games. This is what Walton (1990) calls pretense games, where rules are implicitly governed. The pretense stipulations implicit within a framework define normatively appropriate and inappropriate moves (e.g., Walton, 1990). This is also clear in the pretense games of Habbo Hotel.

Habbo Hotel creates a framework of interaction and visual design that appears to encourage children to play and create new narratives, values, and forms of play, with players creating their own norms and values. In a personal interview (Dutch Game Garden, 2017), Nathalie Korsman (former Habbo Hotel community manager) has revealed that offering children the opportunity to create their own rules and regulations was a deliberate design decision. She also suggested that Habbo empowered children to create not only their own story but also their own society.

With more than 273 million avatars created since August 2012, and with an average of five million unique visitors monthly (Habbo, 2018), this framework appears to be highly successful in engaging children. This is unsurprising; children develop the ability to distinguish between normative social rules and institutional facts at an early age (Rakoczy, 2008). In doing so, they develop a sense of normativity that they can deduce from the context or object they interact with (Rakoczy, Warneken, \& Tomasello, 2008). We, as designers, do not have to decide what is right and wrong; children can do this themselves.

If we want to give children the opportunity to determine their own norms and values, we should try to limit 'placing' our versions in the game (e.g., the artifact children interact with). 
We wished to build upon these kinds of interactions, and we surmised that a certain degree of anonymity might lower the children's barriers to talk about tough subjects.

We decided that our game should present children with the means to express themselves in a safe and non-normative space. Inspired by pretense games and design-for-ambiguity, we set out to work.

\section{The first game}

We started by creating a paper prototype of the game we envisioned. This would help us to quickly determine if our interaction design would support the players' autonomy. We created characters with sticks and playing cards, and these characters were placed in a way that suggested that they were positioned on a tablet. By moving our hand over the character, the other player would perform a corresponding activity with his/her own character.

This vague interaction elicited a verbal response from both parties: 'What are you doing?'; 'I'm stroking your hair.; 'Okay, well, I'm touching your left leg now.' These conversations emerged almost naturally. It appeared that the crude interactions were in need of verbal explanation, as they could mean many things.

The stick figures were translated into an interface design for a tablet. In Figure 8.1, a first sketch of the game presents our initial thoughts on translating the paper prototype into a digital platform. We decided that the game needed a chat channel (as we saw in Habbo) and a thermometer that could be changed to suggest the emotional level of the player. Icons on the site could be tapped and used on the character.

Two characters were designed: a male and a female aged roughly 12 years old. This age was chosen because this is the time when most children enter puberty and become sexually active. The characters were situated from the front, and special attention was paid to designing a character that was culturally undefined. We decided that a mixed-heritage character would help to have more players identify with it.

Being game designers at heart, we figured that we needed to educate the players in the use of the icons. One way to do this is to scaffold the learning (Deen, 2015). This can be achieved by an 'unlocking mechanism'. In Figures 8.3 and 8.4, the unlocking mechanism is visible at the bottom of the screen. Over time, or after a certain number of interactions, players could unlock the icons, creating a full-fledged experience. However, if the 'stop button' 


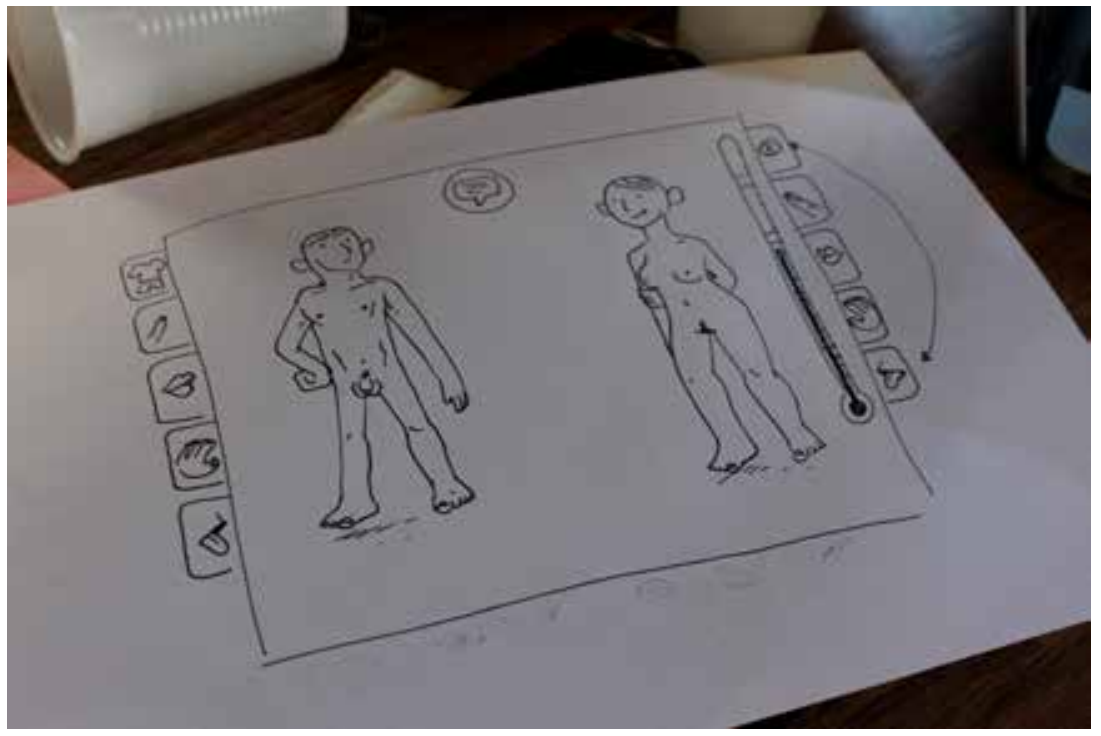

Figure 8.1: First sketch of VilDu?!
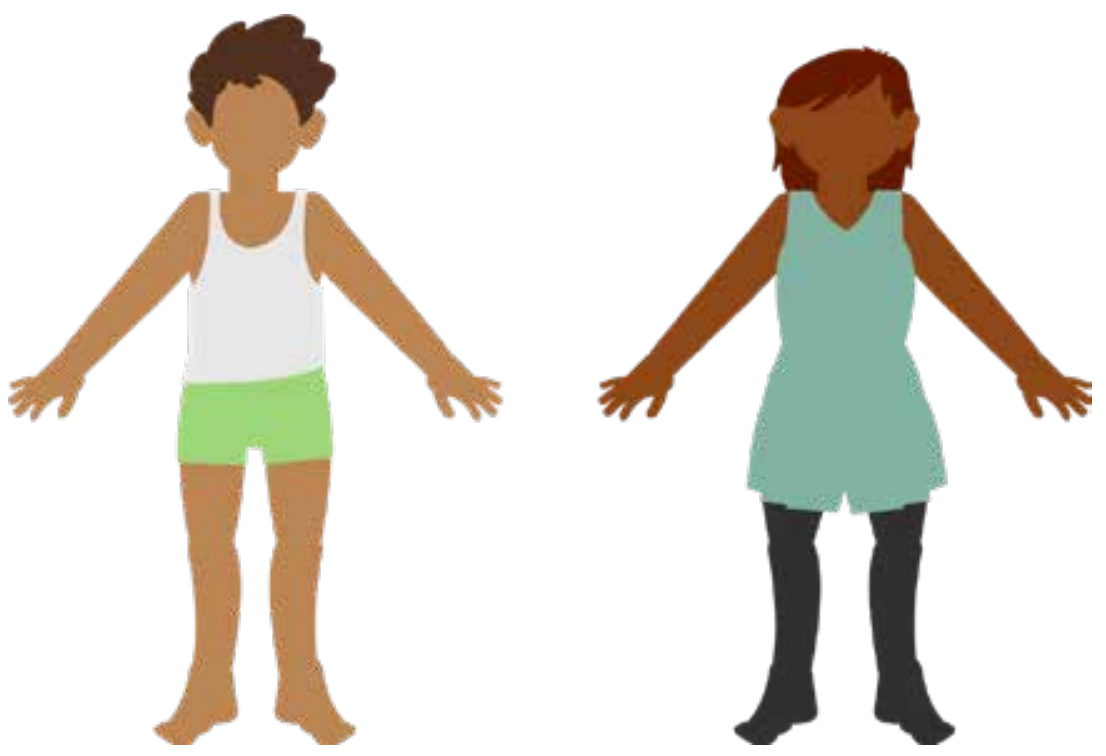

Figure 8.2: Initial design of two characters. 


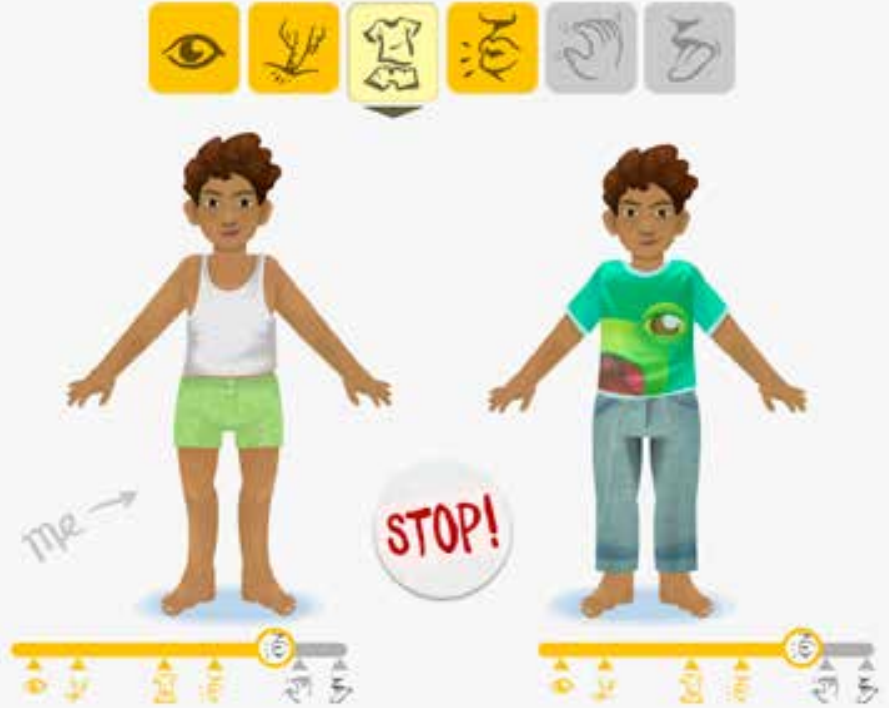

Figure 8.3: Final design of VilDu?! after the Lyst game jam.

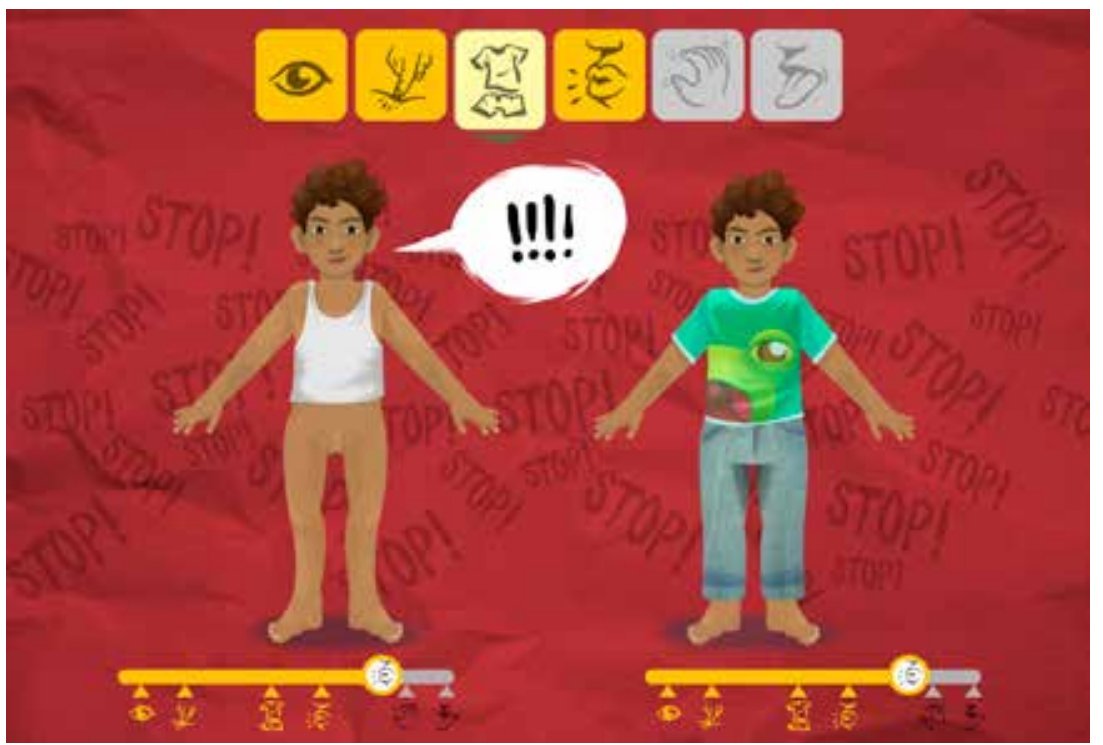

Figure 8.4: After hitting the stop button, unclocked acts could be locked. 
was hit, the screen flared red (see figure 8.4). This would set the other player a couple of steps back in terms of unlocking the icons.

We decided that saying stop does not always mean that intimate partners want the entire intimate act to end. They may just wish not to take part in a particular activity. It will become clear why this implicit norm (as designers, we depicted what 'no' meant) was deleted in the final version of the game. This iteration and some other design decisions were prompted by expert reviews. Notably, the working title VilDu?!, which is Danish for 'Would you?!' stuck and is still used as the title.

\section{Expert reviews}

After the game jam, we brought the game to the attention of Frank Lips' colleagues at De Rading. De Rading is a mental-health institution in the Netherlands that specializes in family therapy. This means that they take the whole system - family members, friends, housing, school, etc.-into account when deciding how they can help clients.

After winning an innovation competition set by KF Heinfonds, meetings were scheduled at De Rading to discuss game iterations. At first, the therapists wanted to add features to the game. Figure 8.5 shows the proposed additions of icons and 'unlock' features. The therapists wanted the ability to move characters closer to one another and expressed a wish to include a way to change the facial expressions (angry, happy, sad, afraid). The simple tool slowly transformed into what Tim Pelgrim began to call a 'flight cockpit', with all these bells and whistles making it hard to understand the game's purpose, interactions, and capabilities.

\section{Further development and iterations}

In the end, only the addition of more characters made it into the final version. We created four different age groups for budgetary reasons: we created characters for two genders for the age ranges 8-12, 15-20, and 25-45. As De Rading seldom deals with $5^{0}+$ females in sexual abuse cases, this last age range is only portrayed as male.

It took three more meetings with the therapists to bring the game to its core functionality, which can be seen in Figure 8.6.

The number of icons was reduced to an eye, a hand, clothing off, clothing on, a mouth, genitalia, and buttocks. The genitalia icon was particularly debated. In order to limit the amount of icons to basic needs, players could only choose the vagina if their character was a female. Similarly, the penis 

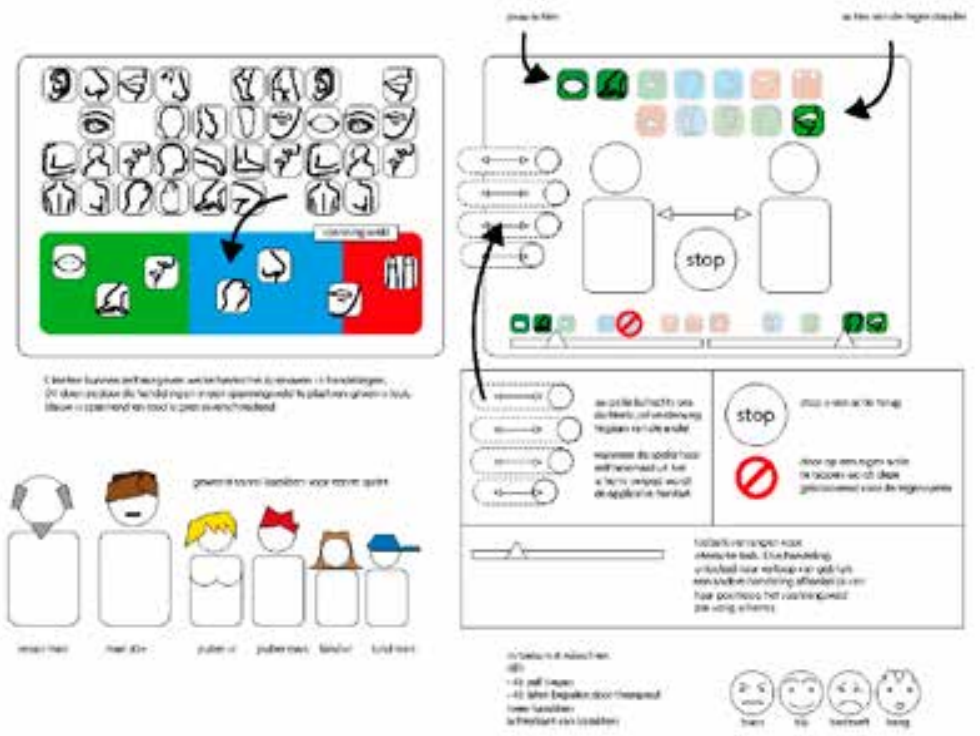

Figure 8.5: Additions to VilDu?! proposed by therapists.

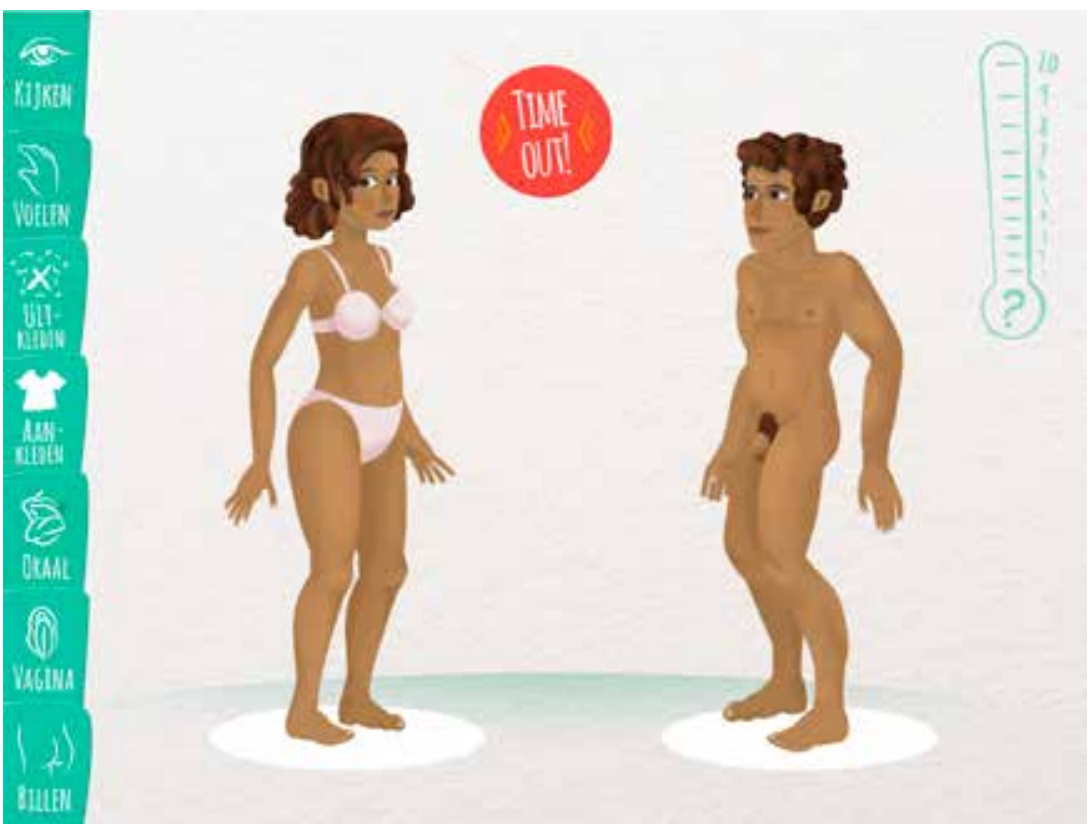

Figure 8.6: The final version of the game took away the captions under the icons. 

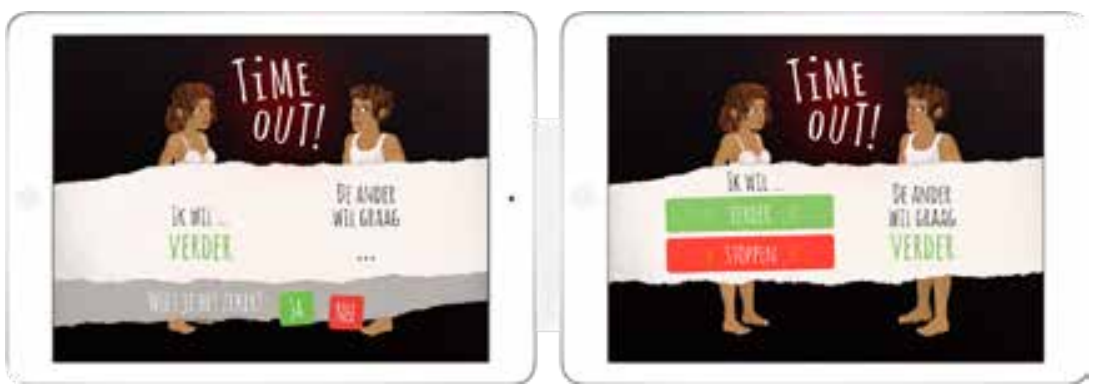

Figure 8.7: When hitting time-out, both screens would 'freeze'. Only if both players agree to carry on does the play continue.

was only available to male characters. This limited the interactions to a rather traditionally gendered approach. In the final version, both the penis and the vagina were available to all genders.

In order to make the game less normative, the unlock mechanism was deleted. This decision was taken on the advice of the therapists, who expressed concern about its use during a conversation. For example, what would happen to a conversation about a traumatic experience if clients needed to wait until an icon was unlocked? This would stall the conversation instead of improving it.

The 'stop' button appeared to become obsolete with the omission of the unlock bar. However, since we agreed that we should always be able to halt an intimate situation, the stop button was given a new purpose: by hitting this button, now called 'time-out', the screen freezes; only when both players agree to continue does the game return to its original state.

The thermometer was brought back, not as an interactive element but more as a visual means to initiate a conversation about the emotional state of the client. As such, the thermometer is just a picture on the screen that clients can use to depict their level of emotional experience.

In the final version, we got rid of the texts below the icons as well. Again, these texts suggested too much. If we wanted children to tell their own story, we should avoid directing them with textual phrases.

\section{First implementation of VilDu?! in therapy sessions}

When the game was in its final stages, Lips tried it in therapy sessions. An example is the case of an eight-year-old, learning-disabled girl who was sexually abused by her neighbor. In conversations, it became clear that she had not yet told the full story. When prompted, she clearly stated that 'not everything was said'. 


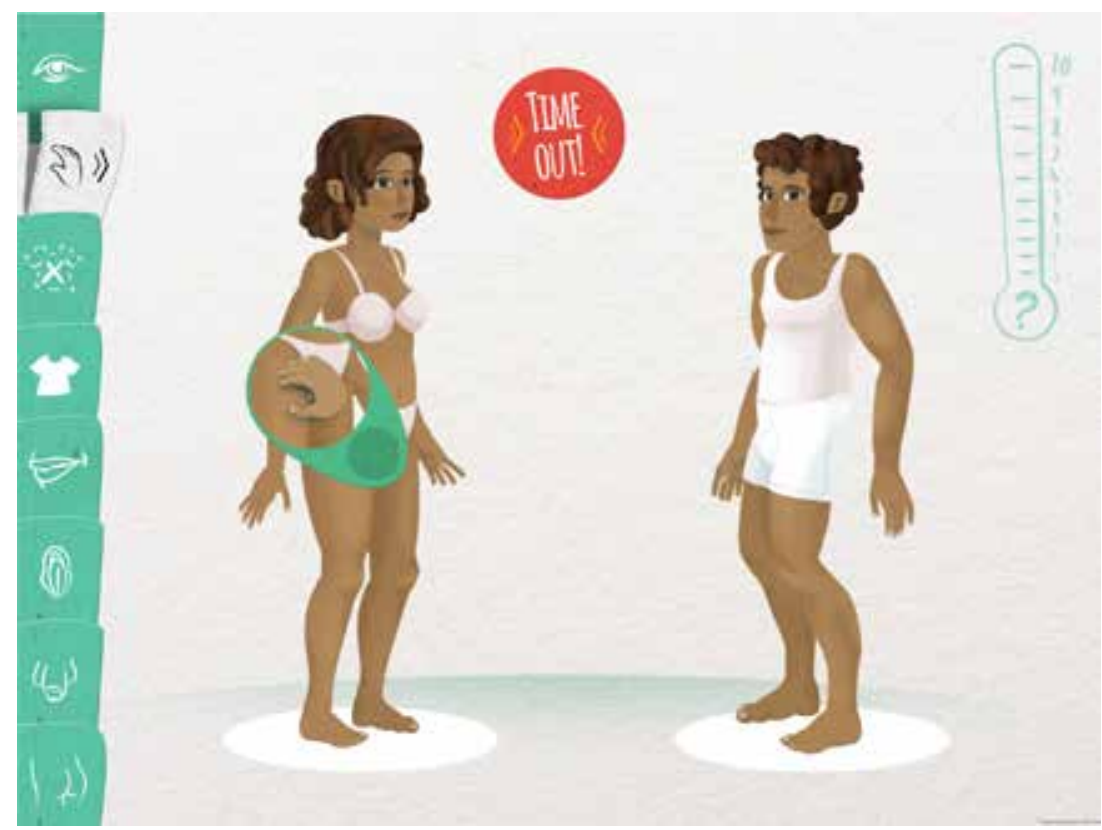

Figure 8.8

The therapist introduced her to VilDu?! in their third session. They played around with the game for a minute or two. She thought it was very funny to undress the older man, stating:

'Ha! Now you are naked!'

After playing around with the game, Lips returned to his initial question: 'What happened, what haven't you told us yet?'

She piped down, stared at the ground and said nothing.

The therapist asked: 'Could you maybe show it... with the tablet?'

The girl nodded. She undressed the older man, selected the hand from the game and said:

'Like this, with his fist...'

She moved the hand back and forward, suggesting that the man was masturbating. 
The girl did not know how to explain what had happened. Both her understanding and her vocabulary fell short. She literally did not have the words to describe masturbation. With VilDu?!, though, she could demonstrate it instead.

Traditionally, the therapist works on building trust between him/herself and the client. Lips needs to develop a shared vocabulary about sexual acts and intimacy. With VilDu?!, he accomplished in five minutes what would normally have taken three weeks.

In other examples, VilDu?! helped a Syrian girl to overcome the Dutch language barrier, while a 15-year-old boy shared information about his sexual relationship with his younger brother. Lips was struck by the speed and ease of these sessions. Where children typically slam shut and discontinue the conversation, VilDu?! appeared to help them to overcome their initial restraint.

Although Lips had various success stories, the adaptation of the app by colleagues and other professionals was limited. This changed when the game won a Dutch game award for Best Serious Game in November 2017. The recognition and appreciation of VilDu?! as an innovative and valuable game for therapy convinced the board of directors to invest more time and money into its implementation.

A team of professionals therefore actively started to look for ways to create more attention and capacity for VilDu?!. This resulted in a collaboration with Utrecht University, which will commence a literature study to formulate a research question in April 2019. The same institution will be applying for several grants for a validation study on the game. The University of Antwerp will perform research on the game as well, and various institutes that work with intellectually challenged clients have expressed an interest in using the app. Finally, the police force for sexual delinquents would like to explore how VilDu?! could help both victims and perpetrators.

Thus, four years after the initial development of VilDu?!, it appears the game will be tested, used, and validated in real-life settings, hopefully helping more victims of sexual abuse.

\section{Lessons learned from the design process}

VilDu?! was developed while working on a project called Games [4Therapy]. It presented us with various insights into the development of therapeutic games. One of the most important design lessons we learned is to focus on something small: Designers could look for something small and easy to relate to instead of problematizing the disorder as something difficult. 
The development of VilDu?! presented us with various insights into the development of persuasive therapeutic games.

In hindsight, we learned three lessons from our design experience:

A. Subtilize the problem: Identify (with) one aspect you can relate to.

B. Create a non-normative play space.

C. Design for ambiguity, which can stimulate conversation.

\section{A. Subtilize the problem}

By subtilizing the problem, I mean that, even though sexual abuse and rape may seem to be something that does not concern you, this is not necessarily the case. Everyone who has been in an intimate relationship has had his/ her boundaries transgressed in smaller or bigger ways. Tapping into those experiences will help in the design of something that connects to them. By subtilizing the problem, I mean making the traumatic experience of someone else smaller and easier to relate to. This helps you to understand the experience. More interestingly, it may also enrich the design process with your personal input.

\section{B. Create a non-normative play space}

VilDu?! is, in essence, a non-normative game. Everything is possible without us (as designers) having preconceptions about what is right or wrong. For example, players in VilDu?! can choose any character, from young to old, from male to female. They can engage in any type of relationship in sexual or intimate play. The game does not stipulate which relationship is most 'healthy'. Instead, it suggests that every sexual relationship is okay, as long as you are able to say no. As a result, the game does not demonize the players but may open up discussions about sex instead.

\section{Design for ambiguity}

Lastly, VilDu?! is a rather explicit game. You can undress characters until they are completely naked. The action icons are based on the physical parameters of the characters. Among other things, there is a vagina, a penis, a hand, a tongue, and a mouth. These are rather explicit. However, the use of the icons has various connotations. It is not the game that explains how to interpret play; it is the player who gives meaning to the interaction. A hand on an arm could mean stroking and caressing it. However, it can also be used to slap or pinch someone. This ambiguity invites players to express 
what they are doing, helping them in small steps to verbalize what happened or what they wanted to occur.

\section{Conclusion}

The design of VilDu!? was a rather enriching experience, not only for us but also for Marcel. For him, this was the very first time that he had told his story from start to end. As a result, he put things in chronological order. This presented him with a clarity he had never experienced before. Furthermore, the understanding of peers and friends helped him to slowly overcome his feelings of shame and guilt. Today, Marcel can handle his intimate relationships better than before.

I am grateful to have been able to facilitate this change and help Marcel to put his feelings into perspective. I am proud that, today, he really understands that saying no is not only okay but is the basis of a healthy intimate relationship.

\section{References}

Bekker, T., Hopma, E., \& Sturm, J. (2010). Creating Opportunities for Play: The Influence of Multimodal Feedback on Open-Ended Play. International Journal of Arts and Technology, 3(4), 325-340. doi:10.1504/IJART.2010.035825.

_., \& Sturm, J. (2009). Stimulating Physical and Social Activity Through Open-Ended Play. In Proceedings of the 8th International Conference on Interaction Design and Children, pp. 309-312. Presented at the Interact, Sweden. doi: 10.1145/1551788.1551869.

Blizzard Entertainment. (2004). World of Warcraft. [Online Game].

Child Safety Research \& Innovation Center. (2008). Sydney Safe-Seeker and the Incredible Journey Home. Retrieved 12 July 2018 from https://www.canada.ca/en/news/ archive/2004/10/sydney-safe-seeker-teaches-children-about-safety-awareness.html.

Deen, M. (2015). G.A.M.E. Games Autonomy Motivation \& Education: How AutonomySupportive Game Design May Improve Motivation to Learn. Eindhoven University of Technology \& Fontys ICT, Eindhoven. Retrieved 11 September 2018 from https://www.academia.edu/10178048/G.A.M.E._Games_Autonomy_Motivation_and_Education_How_autonomy-supportive_game_design_may_improve_motivation_to_learn.

Deen, M., Cercos, R., Chatman, A., Naseem, A., Bernhaupt, R., Fowler, A., Schouten, B., Mueller, F. (2014). Game jam: [4 research]. CHI EA '14: CHI' '4 Extended Abstracts 
on Human Factors in Computing Systems (pp. 25-28). New York, NY: ACM Press. https://doi.org/10.1145/2559206.2559225.

., Nack, F., Haggis, M., Nieuwdrop, E., Vosmeer, M., \& Schouten, B. (2015). Diversity Through Specificy: Design Lessons Learned from the Games [4Diversity] Jams. ACE 2015: Advances in Computer Entertainment Technology. Iskandar, Malaysia: ACM. doi: 10.1145/2832932.2832957.

_ ., \& Schouten, B.A.M. (2014). The Differences Between Problem-Based and Drill \& Practice Games on Motivations to Learn. International Journal of Gaming and Computer-Mediated Simulations (IJGCMS), 7(3). doi: 10.4018/IJGCMS.2015070103. Dutch Game Garden (2017). Q\&A with Nathalie Korsman. Dutch Game Garden. Retrieved 11 April 2018 from https://www.dutchgamegarden.nl/.

GameOven (2013). Friendstrap [Digital Game].

Habbo (2018, November 4). In Wikipedia. Retrieved 5 July 2018 from https:// en.wikipedia.org/w/index.php?title=Habbo\&oldid=867165594.

Hill, A. (2009). Factors Influencing the Degree and Pattern of Parental Involvement in Play Therapy for Sexually Abused Children. Journal of Child Sexual Abuse, 18(4), 455-474. https://doi.org/10.1080/10538710903035214.

Hunter, J.A. (2010). Prolonged Exposure Treatment of Chronic PTSD in Juvenile Sex Offenders: Promising Results from Two Case Studies. Child \& Youth Care Forum, 39(5), 367-384. https://doi.org/10.1007/s10566-010-9108-4.

IJsfontein. (2013a). Can You Fix It? [Digital Game].

- (2013b). Beter in Bed [Digital Game].

IMVU Inc. (2009). IMVU. PC: IMVU. Retrieved 4 July 2018 from http://en.wikipedia. org/wiki/IMVU.

Jarnfelt, P., \& Hasselager, A. (2014). Lyst-Romance, Love and Sex in Games. Retrieved 7 March 2018 from http://www.copenhagengamecollective.org/2014/o6/24/ lyst-a-conference-and-game-jam-celebrating-love-romance-and-sex-in-games/. Karjalainen, S., \& Kyrölä, A. (200o). Habbo.nl. [Online Game].

Kickmeier-Rust, M.D., \& Albert, D. (2009). Emergent Design: Serendipity in Digital Educational Games. In R. Shumaker (ed.), Virtual and Mixed Reality, Vol. 5622, pp. 206-215. Berlin, Heidelberg: Springer Berlin Heidelberg. doi:10.1007/978-3-642-02771-0_24.

Laurel, B. (2004). Design Research: Methods and Perspectives. Cambridge, MA: MIT Press.

Linden Lab. (2003). Second Life [Online Game].

McMahon, L. (1992). The Handbook of Play Therapy. London/New York: Routledge. Norman, D.A. (1990). The Design of Everyday Things. New York, NY: Basic Books.

Piaget, J. (1964). Part I: Cognitive Development in Children: Piaget Development and Learning. Journal of Research in Science Teaching, 2(3), 176-186. https://doi. org/10.1002/tea.366oo20306. 
Rakoczy, H. (2008). Taking Fiction Seriously: Young Children Understand the Normative Structure of Joint Pretence Games. Developmental Psychology, 44(4), 1195-1201. https://doi.org/10.1037/0012-1649-44.4.1195.

_., Warneken, F., \& Tomasello, M. (2008). The Sources of Normativity: Young Children's Awareness of the Normative Structure of Games. Developmental Psychology, 44(3), 875-881. https://doi.org/10.1037/oo12-1649.44.3.875.

Salen, K., \& Zimmerman, E. (2003a). Rules of Play: Game Design Fundamentals. Cambridge, MA: MIT Press. . (2003b). This is Not a Game: Play in Cultural Environments. Proceedings Digital Games Research Conference 2003, 4-6 November 2003, University of Utrecht. Retrieved 18 July 2018 from http://www.digra.org/digital-library/ publications/this-is-not-a-game-play-in-cultural-environments-2/.

Spiegel, E., Murphy, B., \& Spiegel, E. (2011). Snapchat [App].

Sutton-Smith, B. (1997). The Ambiguity of Play. Cambridge, MA: Harvard University Press.

Ternovskiy, A. (2009). Chatroulette. [Online Game].

Toca Boca. (2017). Toca Life: Hospital. [iOS Game].

University of the Sunshine Coast (2012). Orbit. [PC Game].

Van Berlo, W. \& Van Beek, I. (2015). Whitepaper Seksuele Grensoverschrijding en Seksueel Geweld. Utrecht: Rutgers Movisie.

Walton, K.L. (1990). Mimesis as Make-believe: On the Foundations of the Representational Arts. Cambridge, MA: Harvard University Press.

Yancey, C.T., Hansen, D.J., \& Naufel, K.Z. (2011). Heterogeneity of Individuals with a History of Child Sexual Abuse: An Examination of Children Presenting to Treatment. Journal of Child Sexual Abuse, 20(2), 111-127. doi:10.1080/10538712.2 011.554341 .

\section{About the authors}

Menno Deen is a designer/design researcher who explores game design in new and weird contexts. He is the co-founder of Lapp. By creating games with professionals, students, and non-game developers, Menno builds bridges between industries, disciplines, and people to foster the inclusion of new perspectives and domains into the game industry.

Eline Muijres is Board Member of the Games [4Diversity] Foundation and Co-founder of the Dutch game website Laadscherm.nl. Eline previously worked at Dutch Game Garden, Game Oven, and Monogon on Bounden, Jelly Reef, and Interloper, doing PR, marketing, and production. 


\title{
9. Designing for Persuasion through Embodied Experiences in Virtual Reality
}

\author{
Sun Joo Ahn
}

\begin{abstract}
Embodied experiences in virtual reality (VR) involves the reproduction of sufficiently realistic sensory information so that users are able to see, hear, and feel experiences as if they are going through them at the moment. A growing body of literature evinces that the effects of these virtual experiences carry over into the physical world to impact attitudes and behaviors in the physical world. Underlying mechanisms of embodied experiences that produce these outcomes are discussed in the context of media affordances or interactions between novel attributes of VR and user perceptions of them. Design implications to maximize persuasive effects are examined and illustrated with case studies. Finally, the limitations of embodied experiences are considered using the efficiency framework.
\end{abstract}

Keywords: virtual reality; embodied experiences; media affordances; persuasive effects

\section{Introduction}

Immersive virtual environments are digitally rendered spaces offering sensory-rich simulations that allow users to experience mediated events in the virtual world as they might in the physical world (Blascovich \& Bailenson, 2011). Commonly referred to as virtual reality (VR), these highly immersive systems use digital devices to deliver embodied experiences - the reproduction of sufficiently realistic sensory information so that users are able to see, hear, and feel experiences as if they are going through them

Hera, T. Dela, J. Jansz, J. Raessens, B. Schouten, Persuasive Gaming in Context. Amsterdam: Amsterdam University Press, 2021 DOI 10.5117/9789463728805_CHO9 
at that moment (Ahn, Bailenson, \& Park, 2014). The concept of VR is not new - in fact, the discussion and scholarship of virtual environments has a decades-long history (Lanier \& Biocca, 1992; Rheingold, 1992; Sutherland, 1968). However, the costs associated with VR were prohibitive in its earlier years, confining the technology to sophisticated research laboratories and high-tech facilities. With a number of large corporations developing proprietary devices for VR systems, consumers are now able to purchase VR devices at common retail or electronic stores at an affordable price point. Now that VR has the potential to become a ubiquitous, everyday technology, discussions on how VR experiences may alter and transform users' ways of thinking and behaving are timely and imperative.

\section{Using embodied experiences to impact attitudes and behaviors}

Embodied experiences in VR are uniquely positioned to impact attitudes and behaviors because of their ability to mimic direct experiences in physical worlds by providing users with a broad spectrum of simulated information. A growing body of literature demonstrates that embodying visceral experiences in virtual worlds can shift the way people think, feel, and behave, and that these effects persist and transfer into the physical world to influence attitudes and behaviors over time, after people have left the virtual worlds (Ahn, 2015; Ahn, Fox, Dale, \& Avant, 2015; Ahn, Bailenson, \& Le, 2013). A deeper understanding of why and how embodied experiences can impact users will yield opportunities to harness the persuasive powers of virtual worlds to promote desirable behaviors.

\section{The persuasive power of direct experiences}

Much of the excitement surrounding VR involves how realistically the mediated environment is able to deliver experiences 'just like' the physical world. Articles from both the academic and popular press often compare VR to traditional media platforms such as television or books and note the technology's ability to put users in simulations that make them seem as if they are really ducking bullets in a war zone, swimming with marine life in the sea, or exploring crevices on Mars. Why are people so interested in creating authentic and realistic experiences in VR, and how do these impact users?

Social scientists have noted for decades that direct experiences, wherein the person has first-hand contact with the event, seem to have meaningful 
effects on attitudes and behavior change compared to indirect experiences, wherein the person has secondhand contact with the event. As direct and indirect experiences are encountered in different formats, they are encoded in the mind differently and therefore affect attitudes and behaviors in different ways (Hamilton \& Thompson, 2007). As a result, individuals form stronger attitudes, feel more confident about the attitudes formed, and ultimately behave more consistently with those attitudes from direct than indirect experiences (Fazio \& Zanna, 1991; Wu \& Shaffer, 1987). Due to this confidence in the attitudes formed, individuals place greater weight on direct than indirect experiences when making decisions; personal experience of an event makes the information more salient and serves as a more significant point of reference than a description of the event (Hertwing et al., 2004; Rajecki, 1982). These findings suggest that, when designing for persuasion, experiences that closely mimic first-hand events may be more effective than secondhand depictions.

Direct experiences provide rich raw materials for constructing mental schemata that are to be later activated and recalled when individuals encounter or think about similar stimuli (Barsalou, 2009). This implies that, given sufficient perceived realism and authenticity, embodied experiences in VR may continue to influence attitudes and behaviors outside the virtual world when individuals encounter similar stimuli in the physical world. So, designing for persuasion in VR involves the consideration of sustained effects that last over time, which might be a more cost-effective approach to persuasion than using traditional media, which yields effects that dissipate relatively faster (Ahn, 2015; Ahn et al., 2014). The ability of embodied experiences to mimic direct experiences in the physical world becomes even more meaningful when taking into consideration VR's capacity to deliver experiences that are difficult or impossible to encounter in the physical world. For these events, embodied experiences in VR provide a useful platform to persuade people to consider risks or future negative consequences that would be difficult to communicate otherwise.

\section{Mechanisms of persuasion through embodied experiences: an affordance-based approach}

VR offers a wide range of features that distinguish the platform from traditional channels. However, features alone, without considering user perceptions and preferences, may not be enough to persuade. Consider, for instance, digital devices with features that are not well understood and are 
therefore rarely used by individuals: Although the feature exists, if the user doesn't take advantage of it, it fails to affect the user in any way. On the other hand, the potential for using devices in a certain way wouldn't exist at all without the features themselves. As such, exploring the 'relational structure' of affordances - the dynamic interaction between users and features mutually deciding the capabilities and limitations of technologies-is critical (Evans, Pearce, Vitak, \& Treem, 2017). How do these affordances promote or hinder persuasive outcomes through embodied experiences?

\section{Presence}

When VR uses digital devices to produce realistic sensory information, users may temporarily forget that they are in a mediated world and feel as if they have genuinely visited a different space (Lombard \& Ditton, 1997; Slater \& Wilbur, 1997). This feeling of 'being there' in the virtual world during an embodied experience is referred to as presence (Biocca, 1997). Although presence is often discussed in the context of VR, the ability of users to feel present in a believable illusion of mediated environments can be applied to other platforms as well, including video games (Tamborini \& Skalski, 2006), television (Kim \& Biocca, 1997), and books (Schubert \& Crusius, 2002).

Presence is applicable across different platforms because this affordance is the result of dynamic interactions between the modality features that support the delivery of sensory information and users' psychological willingness to mentally construct a believable illusion of authentic experiences (Lee, 2004). Conceptually, media features alone are insufficient to induce feelings of presence. So, Eva might feel that she was really walking on the surface of Mars in a virtual simulation, but Teresa might feel that the same simulation of Mars was not sufficiently realistic and will still feel physically situated in her own living room.

However, the reason that the concept of presence is commonly discussed in the context of VR is because its features facilitate the delivery of multiple layers of sensory information that envelop the user without him/her having to actively engage in mental imagery. By surrounding the user with multiple cues, such as visual, aural, and tactile information, VR may serve as the facilitator of presence, given equal individual capacity and the motivation to perceive presence. This is why the bulk of scholarly work on VR associates greater levels of perceived presence with higher levels of interactivity, richness, and immersion. As a consequence, more technologically sophisticated virtual environments are more conducive to higher perceptions of presence than virtual environments that provide fewer perceptual cues. 
Particularly relevant to embodied experiences is the dimension of spatial presence, which refers to the extent to which the user feels that the objects and events encountered in the mediated environment are authentic (Lee, 2004; Lombard \& Ditton, 1997). There are several measures that assess the perception of spatial presence (Schubert, Friedmann, \& Regenbrecht, 2001; Witmer \& Singer, 1998). Commonly used items gauge the extent to which users feel that they can reach out to touch and move objects in the mediated space (possible actions), or the extent to which they feel as if they are surrounded by their environment (self-location), which illustrates the focal points of the construct (Wirth et al., 2007). The bulk of the scholarship around spatial presence has focused on visual and aural cues, perhaps because these are the dominant sense modalities and are what drive our perceptions and understanding of the world that surrounds us. With the development of haptic controllers, studies of tactile cues are on the rise, but the roles of tactile, olfactory, and gustatory cues in the construction of spatial presence remain largely underexplored.

As the development of virtual worlds has historically been spearheaded by video game industries, discussions of spatial presence in virtual worlds have focused on the domains of user engagement or the enjoyment of the mediated content (Skalski, Tamborini, Shelton, Buncher, \& Lindmark, 2011; Vorderer, Klimmt \& Ritterfeld, 2004). However, features that construct embodied experiences with a high spatial presence go above and beyond mere entertainment, impacting user attitudes and behaviors in the physical world for some time after the virtual experience. If direct physical experiences wield greater influence for attitude and behavior change, virtual simulations that elicit a high spatial presence are able to more closely mimic first-hand experiences and may consequently serve as a more powerful tool of persuasion than media channels that provoke a relatively passive reception from users (Ahn et al., 2015; Kim \& Biocca, 1997; Lombard \& Snyder-Duch, 2001).

\section{Acceleration of time}

In the physical world, time is a structured and continued progression, forever ticking forward at a strict, predetermined pace. In the virtual world, time becomes more fluid. VR facilitates the ability to depict an accelerated progression of time that moves either forward or backward with the help of computer graphics and devices that replicate sensory information. VR can be used to depict incremental levels of change dynamically in a matter of 
minutes, allowing users to embody the changes over time in a way that's impossible in the physical world. For example, environmental damage happens over such an extended period of time that individuals rarely have the opportunity to experience the future negative outcomes of their present behaviors. In VR, however, users may see, hear, and feel the grave dangers of environmental damage in just minutes: experiencing deforestation taking place before their eyes (Ahn et al., 2014) or watching marine animals die as a result of an acidifying ocean (Ahn et al., 2016).

This affordance enables virtual simulations to be more potent and effective tools of persuasion than traditional media platforms. People often underestimate or overestimate the impact of future experiences, thinking of the future as an isolated event, independent of the past and present, and basing their forecasts of the future on successful plans and scenarios rather than on accurate past results (Kahneman \& Lovallo, 1993). Individuals are also likely to have an unrealistic level of optimism in conceptualizing distant future events (Weinstein, 1980). By portraying accelerated depictions of events dynamically, VR provides a means to concretely connect the past, present, and future so that causal relationships become lucid. When it becomes clear that present choices and behaviors lead to negative future consequences, it becomes easier to persuade individuals to change their present behaviors to avoid the virtually experienced negative consequences in the future because the accelerated time in the simulation is likely to elicit a sense of urgency.

\section{Shared experiences through perspective-taking}

Another unique affordance of embodied experiences in VR is the ability to share sensory-rich experiences from a first-person viewpoint. This provides the user with the opportunity to take the perspective of another entity (human or non-human), to see, hear, and feel as it would. Perspective-taking is the mental simulation of a situation that involves placing oneself in the shoes of another via imagination (Batson et al., 1997). In the physical world, perspective-taking is a cognitively effortful task, requiring individuals to invest substantial cognitive resources as they attempt to situate themselves in the position of another being. VR assists in reducing the burden of effort by surrounding the user with layers of sensory information from the perspective of the other entity. So, individuals may see the world through the eyes of a visually impaired person to gain first-hand experience of living with a disability. The affordance of shared experiences through 
perspective-taking may engender higher levels of attitude and behavior change because experiencing an event first-hand leads to high involvement, and involvement often serves as a predictor of persuasion (Petty \& Cacioppo, 1981). Empirical evidence supports this supposition: People who experienced the virtual simulation of living with a visual impairment (e.g., red-green color blindness) actively helped those with disabilities for twice as long as those who were asked to just imagine what it would be like to live with a disability (Ahn et al., 2013). The results also suggested that VR simulation might be more helpful for some than others, depending on individual differences in the motivation for engaging in perspective-taking.

\section{Case studies of persuading through embodied experiences: design applications}

Design elements may be leveraged in VR to heighten the impact of these affordances on persuasion. As embodied experiences differ in nature from traditional practices of media consumption, understanding how these affordances can be translated into design applications in VR is critical. Some case studies that illustrate how these designs may be integrated into the development of the virtual simulation for attitude and behavior change are presented next.

\section{Communicating risk}

Risk is defined as 'things, forces, or circumstances that pose danger to people or to what they value' (Stern \& Fineberg, 1996). The anticipation of, and the desire to avoid, negative consequences as a result of the risk event motivates behavior change for self-protection reasons (O'Connor, Yarnal, Dow, Jocoy, \& Carbone, 2005). Several challenges to risk communication pose difficulties in motivating individuals to change their present behaviors to avoid future negative consequences: People tend to underestimate the influence of situational variables at the time of prediction (Loewenstein, 1996). For example, someone might overestimate his/her will power when it comes to reducing their soft drink consumption immediately following exposure to information on the negative health consequences of sugarsweetened beverages, with the will power subsiding significantly when he/she comes across a vending machine on a hot day. Another challenge of risk communication is that the general public often fails to recognize 
issues when they are not directly or immediately observable. So, although ocean acidification reduces the $\mathrm{pH}$ of the earth's oceans and leads to a grave disruption of the entire marine system, individuals perceive the risk of this disruption to be low because they rarely have the opportunity to see personally the consequences of ocean acidification on marine life.

VR simulations can present changes concretely between the present and the future in mere minutes, and this virtual acceleration of time can help bridge psychological distances between present behaviors and future consequences (Ahn, 2015). The design implementation of accelerating time can involve a variety of techniques. One way to indicate the passing of time is to display a means to tell time, such as a calendar, clock, or the rising and setting of the sun, depicting the rapid progression of time through these objects. So, avatars that instantly and dynamically gain weight as a result of poor dietary choices have been used in virtual simulations to depict future negative health consequences - the moment a user makes an unhealthy food choice in the virtual world, the avatar can be designed to display noticeable changes in body shape and general health (Ahn, 2015; Fox, Bailenson, \& Binney, 2009).

Another way to visualize the acceleration of time is to set 'start' and 'end' points with a virtual human's face and demonstrate how it dynamically transitions from one point to another, for instance, by way of an aging face. FaceApp, a recently launched smartphone application, allows users to easily experience what their face might look like 30 years in the future by taking a selfie and using slider bars to 'age' the face.

Yet another design strategy to demonstrate the acceleration of time is to depict how the environment shifts over time-a dynamic, fast-forwarded rendering of change. So, in a simulation of ocean acidification, participants begin the virtual experience in a clear, underwater world with fish and other marine animals. In a matter of minutes, due to environmental pollution, the participants vividly experienced the marine animals dying and their own avatar bodies disintegrating in a rapidly acidifying body of water (Ahn et al., 2016).

Other design elements can heighten the perception of presence to engender risk perceptions effectively. Although direct experiences are more effective than indirect experiences in encouraging people to become involved with a risk issue, in most cases it would be illogical or even fatal to suggest that people should gain first-hand experience with the risk issue: Imagine suggesting that someone experience cancer or an earthquake. To simulate first-hand experiences of risk events, first-person perspectives that give users the viewpoint of the protagonist are often adopted. So, 
AccuWeather, a weather information provider, has designed a 360-degree video application that places users at the center of extreme weather events, such as tornados or thunderstorms. Allowing users to see, hear, and feel multiple elements of the simulation is likely to increase their perceptions of presence, leading to greater engagement and involvement with the risk issue at hand.

\section{Promoting behaviors}

Social science research has demonstrated that exposure to information alone does not change behavior; instead, personal experiences of prior success are one of the strongest drivers of behavior change (Bandura, 1977). In addition, studies have confirmed intrinsic ties between bodily actions and cognitive processes (Feldman \& Narayaman, 2004), wherein motor actions can encourage cognitive processing so that later, when the mind is just thinking about the situation, the body is more prepared to execute the learned actions.

These findings suggest that embodied experiences with opportunities for physical interactions with the mediated space may serve as drivers of behavior change. VR simulations can be designed so that users can simulate and practice recommended behaviors in the virtual world to gain the confidence required to carry out the same behaviors in the physical world. Some studies have demonstrated that playing prosocial video games and practicing prosocial behaviors lead to such behaviors in the physical world (Gentile et al., 2009; Greitemeyer \& Osswald, 2010). Exercising in the virtual world has also led to more exercising in the physical world (Fox \& Bailenson, 2009).

Presenting vicarious reinforcement in VR is another effective design approach for behavior change. Observing another person's success or failure is a strong impetus for behavior change (Bandura, 1977). Avatars and agents allow individuals to vividly observe the future consequences of present choices that may be difficult to observe in the physical world. For example, exercising behavior is often difficult to sustain over time due to delayed gratification-exercising, regardless of its intensity, is unable to produce immediately observable changes. In VR, avatars and agents can be designed so that the benefits of exercise are immediately observable through changes in physical appearance (e.g., becoming slimmer with more avatar movements) or the movements of the digital representations (e.g., the avatar is able to move faster and gains more agility). If the user's 
behavior is connected to a clearly observable positive or negative change in his/her avatar, the vicarious reinforcement through the avatar may serve as an impetus for behavior change in the physical world.

Finally, VR provides the capacity for users to implement naturalistic movements when interacting in the mediated environment. Users are able to look around the virtual world as they would the physical world and can reach out to grab objects in a similar way. Scholars have argued that using naturalistic controls to engage with the virtual world creates more complete and accurate mental models than traditional means of interacting with mediated worlds, such as button-presses (Tamborini \& Bowman, 2010). Mental models created from direct experiences are more concrete and detailed than those produced from indirect experiences or descriptions (Hamilton \& Thompson, 2007). Therefore, virtual experiences that closely mimic physical ones are likely to create concrete mental models that are rich in detail. When individuals are later exposed to situations similar to the one they experienced in the virtual world, the mental models are likely to become activated and impact their behaviors. And the more detailed these models are, the more likely they are to direct behavior change (Bandura, 2001; Barsalou, 2009).

\section{Experiencing brands}

VR offers opportunities to provide vivid and engaging brand experiences that take advertising theory and practice above and beyond the traditional delivery of branded messages that typically relies on unidirectional communication with audiences. Embodying a branded experience blurs earlier conceptualizations of the roles of the sender and receiver of the messagebecause the experience is highly interactive, consumers become actively engaged in the persuasion process and even take part in the construction of the message.

So, consider a branded experience from Kia, the automotive manufacturer, which uses VR to deliver a test-drive simulation to potential consumers. Consumers are now able to directly interact with the car without ever stepping inside a dealership, feeling the responsiveness and handling of the vehicle and even the vibration as the tires hit the road. Such branded experiences integrate the self in the mental model of the brand as consumers remember their own arms and hands touching and moving objects in the virtual world, thereby endorsing the brand or product. Self-endorsing, a novel advertising strategy afforded by VR wherein the self is actively integrated 
within a branded experience (Ahn \& Bailenson, 2011), favorably impacts brand preferences. Studies have demonstrated that virtually associating the self and the brand leads to positive attitudes and purchase intentions for the brand, even if the consumer has never seen it before (Ahn \& Bailenson, 2011).

Consequently, interactive design elements that explicitly involve the self within the branded message is more likely to engender favorable brand attitudes than a passive viewing experience. So, consider building in a mirror to allow consumers to view a reflection of their self-avatar as they try out a product in the virtual world. Alternatively, offer a personalized product in the virtual world, engraved with the consumer's name. Better yet, have the consumer experience the benefits of the product during the virtual experience by driving the car, wearing the dress, or sitting on the new sofa. The virtual interaction with the product and brand is likely to form strong associations between the self and the brand that transfer to the physical world.

On the other hand, virtual experiences that are novel and stimulating yet irrelevant to the brand and its image may not be as effective. An earlier study looking at the impact of the thematic relevance of advergames and brand attitudes made a similar point: Advergames should be designed so that the theme and content of the game are related to the sponsoring brand in order to elicit favorable brand attitudes as a result of engaging in game play (Wise, Bollks, Kim, Venkataraman, \& Myer, 2008). Following the same logic, when a virtual experience is novel and exciting but irrelevant to the brand, users are likely to enjoy the simulation, but the enjoyment may not lead to favorable brand outcomes. Regardless of the platform, all communication efforts should aim to be consistent with and relevant to the sponsoring brand and its image.

\section{Limitations of embodied experiences}

In communication scholarship and practice, media platforms that provide a rich array of sensory and content cues (e.g., VR) have been favored over 'leaner' platforms (e.g., text-based), with the anticipation being that richer media will lead to better quality communication. As a result, a high presence has often been considered to be a unilaterally positive outcome of media platforms. In contrast, little effort has been invested in exploring the potentially negative outcomes that may result from sensory saturation or information overload in identifying the boundary conditions of embodied experiences. 
Rather than a general, linear association between presence and performance, the efficiency framework takes on a more nuanced approach to understanding the influence of presence by recognizing that different media are appropriate for different tasks (Walther, 2011). For instance, multichannel platforms such as VR have been shown to be better than mental imagery or the imagination when it comes to adopting the perspective of another person (Ahn et al., 2013). Conversely, earlier work demonstrates that face-to-face or multi-channel interactions do not always outperform mediated, indirect communication with platforms that provide fewer cues (Mühlfelder et al., 1999).

VR may be efficient and effective for inducing presence, engaging the user, and rendering an experience enjoyable (Skalski et al., 2011), but this engagement may distract from the content or information provided, making it potentially less efficient for certain goals such as tending to specific facts or recalling the information presented. This presents an interesting paradox wherein the preferred and enjoyed communication platform may not always be the most efficient for a given task. So, embodied experiences in VR that envelop the user with multiple channels of sensory information may not be an optimal platform for information-dense simulations that are often used in educational contexts. Individuals have limited cognitive resources to allocate in the processing of information delivered via mediated environments (Lang, 200o), and multi-channel stimuli may overload users' cognitive capacities and limit the amount and quality of information decoded, stored, and recalled (Kim \& Biocca, 1997). Complex structural features of a message, such as sound effects and edits, increase the attention paid to process these features and, consequently, few resources are left when it comes to successfully encoding and later recalling the information (Lang, 2000). Indeed, some preliminary studies have demonstrated that presence in VR is associated with reduced cued recall (Ahn, Nowak, \& McGillicuddy, 2017; Bailey et al., 2012).

\section{Conclusion}

With the recent development of stand-alone VR systems and wireless headsets, this technology is poised to continue gaining in popularity and accessibility in the coming years. The affordances of embodied experiences, such as presence and the acceleration of time, espouse unique and unprecedented dynamics between users and system features that encourage attitude and behavior change. Therefore, when properly and carefully 
designed and implemented, the increased use of embodied experiences may be a valuable persuasion tool.

However, embodied experiences may not always lead to desired outcomes and should be designed and implemented after careful consideration of goals and intended as well as unintended consequences. In cases where the goal of the message is high-level information processing and recall, preliminary findings suggest that sensory-rich embodied experiences may deplete valuable cognitive resources and lead to cognitive overload. As a result, it's possible that, although users enjoy the experience of the virtual simulation, they fail to effectively process and retain the information provided. Conversely, in cases where the goal is to engage users and elicit attitude and behavior change through visceral experiences, embodied experiences may be more appropriate than traditional platforms. To enjoy the maximal potential for persuasion offered by embodied experiences in VR, theory-driven designs that carefully take into consideration the constraints of individual differences, situational contexts, and the costs and benefits of implementation are recommended.

Furthermore, the increased availability of VR systems has recently introduced a number of multi-user environments, such as Altspace VR, VR Chat, Rec Room, and High Fidelity. In these environments (i.e., social VR), large numbers of users are able to interact and connect in the same virtual space. Although many of the current embodied experiences offered in VR are solitary, humans are by nature social animals. The future of embodied experiences likely lies in multi-user environments, similar to how video gaming has become a social rather than a solitary activity. As more users gather and interact in these spaces over the years, many affordances of embodied experiences will become reexamined and redefined as virtual worlds gradually evolve into socially networked spaces.

\section{References}

Ahn, S.J. (2015). Incorporating Immersive Virtual Environments in Health Promotion Campaigns: A Construal-Level Theory Approach. Health Communication, 3o(6), 545-556. doi:10.108o/10410236.2013.869650.

\& \& Bailenson, J.N. (2011). Self-Endorsing Versus Other-Endorsing in Virtual Environments: The Effect on Brand Attitude and Purchase Intention. Journal of Advertising, 4o(2), 93-106. https://doi.org/10.2753/JOAoog1-3367400207.

— Bailenson, J.N, \& Park, D. (2014). Short- and Long-Term Effects of Embodied Experiences in Immersive Virtual Environments on Environmental Locus of 
Control and Behavior. Computers in Human Behavior, 39, 235-245. https://doi. org/10.1016/j.chb.2014.07.025.

, Bostick, J., Ogle, E., Nowak, K., McGillicuddy, K., \& Bailenson, J.N. (2016). Experiencing Nature: Embodying Animals in Immersive Virtual Environments Increases Inclusion of Nature in Self and Involvement with Nature. Journal of Computer-Mediated Communication, 21(6), 399-419. https://doi.org/10.1111/jcc4.12173.

_, Fox, J, Dale, K.R., \& Avant, J.A. (2015). Framing Virtual Experiences: Effects on Environmental Efficacy and Behavior Over Time. Communication Research, 42(6), 839-863. https://doi.org/10.1177/oog3650214534973.

, Le, A. M. T., \& Bailenson, J. N. (2013). The Effect of Embodied Experiences on Self-Other Merging, Attitude, and Helping Behavior. Media Psychology, 16(1), 7-38. https://doi.org/10.1080/15213269.2012.755877.

— Nowak, K., \& McGillicuddy, K. (2016). Processing Information as a Body of Coral: The Role of Spatial Presence on Learning in Immersive Virtual Environments. National Communication Association Conference. November 10-13, Philadelphia, PA.

Bailey, J., Bailenson, J.N., Won, A.S., Flora, J., \& Armel, K.C. (2012). Presence and Memory: Immersive Virtual Reality Effects on Cued Recall. Proceedings of the International Society for Presence Research Annual Conference. 24-25 October, Philadelphia, PA. Retrieved 5July 2017 from https://vhil.stanford.edu/mm/2012/ bailey-ispr-presence-memory.pdf.

Bandura, A. (2001). Social Cognitive Theory of Mass Communication. Media Psychology, 3(3), 265-299. https://doi.org/10.1207/S1532785XMEP0303_03

_. (1977). Self-Efficacy: Toward a Unifying Theory of Behavioral Change. Psychological Review, 84

(2), 191-215. https://doi.org/10.1037/0033-295X.84.2.191Barsalou, L.W. (2009). Simulation, situated conceptualization, and prediction. Philosophical Transactions of the Royal Society B, 364, 1281-1289.

Batson, C.D., Polycarpou, M.P., Harmon-Jones, E., Imhoff, H.J., Mitchener, E.C., Bednar, L.L., et al. (1997). Empathy and Attitudes: Can Feeling for a Member of a Stigmatized Group Improve Feelings Toward that Group? Journal of Personality and Social Psychology, 72(1), 105-118. Retrieved 9 March 2018 from https://psycnet. apa.org/buy/1997-02176-oog.

Biocca, F. (1997). The Cyborg's Dilemma: Progressive Embodiment in Virtual Environments. Journal of Computer-Mediated Communication, 3(2). https:// doi.org/10.1111/j.1083-6101.1997.tbooo7o.x.

Blascovich, J. \& Bailenson, J. (2011). Infinite Reality: Avatars, Eternal Life, New Worlds, and the Dawn of the Virtual Revolution. New York, NY: Harper Collins. Evans, S.K., Pearce, K.E., Vitak, J., \& Treem, J.W. (2017). Explicating Affordances: A Conceptual Framework for Understanding Affordances in Communication 
Research. Journal of Computer-Mediated Communication, 22(1), 35-52. https:// doi.org/10.1111/jcc4.12180.

Fazio, R.H., \& Zanna, M.P. (1981). Direct Experience and Attitude-Behavior Consistency. In L. Berkowitz (ed.). Advances in Experimental Social Psychology, 14, 161-202. New York, NY: Academic Press.

Feldman, J., \& Narayanan, S. (2004). Embodied Meaning in a Neural Theory of Language. Brain and Language, 89(2), 385-92. doi: 10.1016/Soo93-934X(03)00355-9. Gentile, D.A., Anderson, C.A., Yukawa, S., Ihori, N., Saleem, M., et al. (2009). The Effects of Prosocial Video Games on Prosocial Behaviors: International Evidence from Correlational, Longitudinal, and Experimental Studies. Personality and Social Psychology Bulletin, 35(6), 752-763. doi: 10.1177/0146167209333045.

Greitemeyer, T. \& Osswald, S. (2010). Effects of Prosocial Video Games on Prosocial Behavior. Journal of Personality and Social Psychology, 35(6), 211-221. 10.1177/0146167209333045.

Hamilton, R.W., \& Thompson, D.V. (2007). Is There a Substitute for Direct Experience? Comparing Consumers' Preferences After Direct and Indirect Product Experiences. Journal of Consumer Research, 34, 546-555. https://doi.org/10.1086/520073.

Hartmann, T., Wirth, W., Vorderer, P., Klimmt, C., Schramm, H., Böcking, S. (2015). Spatial Presence Theory: State of the Art and Challenges Ahead. In Lombard, M., Biocca, F., Freeman, J., IJsselsteijn, W., \& Schaevitz, R. (eds.) Immersed in Media. Cham, Switzerland: Springer International Publishing AG.

Hertwing, R., Barron, G., Weber, E.U., \& Erev, I. (2004). Decisions from Experience and the Effect of Rare Events. Psychological Science, 15, 534-539. https://doi. org/10.1111/j.0956-7976.2004.00715.x.

Kim, T. \& Biocca, F. (1997). Telepresence Via Television: Two Dimensions of Telepresence May Have Different Connections to Memory and Persuasion.Journal of Computer-Mediated Communication, 3 (2). https://doi.org/10.1111/j.1083-6101.1997. tbooo73.x.

Lanier, J., \& Biocca, F. (1992). An Insider's View of the Future of Virtual Reality. Journal of Communication, 42, 150-172. https://doi.org/10.1111/j.1460-2466.1992. tboo816.x.

Lang, A. (2000). The Limited Capacity Model of Mediated Message Processing. Journal of Communication, 5o(1), 46-70. https://doi.org/10.1111/j.146o-2466.2000. tbo2833.x.

Loewenstein, G. (1996). Out of Control: Visceral Influences on Behavior. Organizational Behavior and Human Decision Processes, 65(3), 272-292. https:// doi.org/10.1006/obhd.1996.0028.

Lombard, M., \& Ditton, T. (1997). At the Heart of it All: The Concept of Presence. Journal of Computer-Mediated Communication, 3 (2). https://doi. org/10.1111/j.1083-6101.1997.tbooo72.x. 
— Snyder-Duch, J. (2001). Interactive Advertising and Presence: A Framework. Journal of Interactive Advertising, 1(2), 56-65. https://doi.org/10.1080/152 52019.2001.10722051.

Mühlfelder, M., Klein, U., Simon, S., \& Luczak, H. (1999). Teams Without Trust? Investigations in The Influence of Video-Mediated Communication on the Origin of Trust Among Cooperating Persons. Behavior \& Information Technology, 18(5), 349-36o. https://doi.org/10.1080/014492999118931.

O'Connor, R.E., Yarnal, B., Dow, K., Jocoy, C.L., \& Carbone, G.J. (2005). Feeling at Risk Matters: Water Managers and the Decision to Use Forecasts. Risk Analysis, 25(5), 1265-1275. 10.1111/j.1539-6924.2005.00675.x.

Petty, R.E., \& Cacioppo, J.T. (1979). Issue Involvement Can Increase or Decrease Persuasion by Enhancing Message-Relevant Cognitive Responses. Journal of Personality and Social Psychology, 37, 1915-1926. 10.1037/0022-3514-37.10.1915.

Rajecki, D.W. (1982). Attitudes: Themes and Advances. Sunderland, MA: Sinauer Associates.

Rheingold, H. (1992). Virtual Reality. New York, NY: Simon \& Schuster.

Rizzo, A., Buckwalter, G., Forbell, E., Reist, C., Difede, J., Rothbaum, B.O., Lange, B., Koenig, S., \& Talbot, T. (2013). Virtual Reality Applications to Address The Wounds Of War. Psychiatric Annals, 43(3), 123-138. https://doi. org/10.3928/00485713-20130306-08.

Rosenberg, R.S., Baughman, S.L., \& Bailenson, J.N. (2013). Using Superpowers in Virtual Reality to Encourage Prosocial Behavior. PLoS ONE 8(1): e55003. https:// doi.org/10.1371/journal.pone.0055003.

Schubert, T., \& Crusius, J. (2002). Five Theses on the Book Problem: Presence in Books, Film And VR. In F. R. Gouveia \& F. Biocca (eds.), PRESENCE 2002 - Proceed-

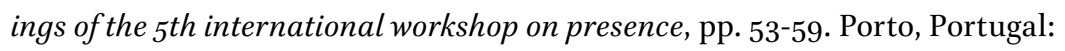
Universidad Fernando Pessoa.

— Friedmann, F., \& Regenbrecht, H. (2001). The Experience of Presence: Factor Analytic Insights. Presence: Teleoperators and Virtual Environments, 1o(3), 266-281. https://doi.org/10.1162/105474601300343603.

Skalski, P., Tamborini, R., Shelton, A., Buncher, M., \& Lindmark, P. (2011). Mapping the Road to Fun: Natural Video Game Controllers, Presence, and Game Enjoyment. New Media \& Society, 13 (2), 224-242. https://doi.org/10.1177/1461444810370949.

Slater, M., \& Wilbur, S. (1997). A framework for immersive virtual environments (FIVE): Speculations on the role of presence in virtual environments. Presence: Teleoperators and Virtual Environments, 6, 603-616.

Stern, P.C. \& Fineberg, H.V. (eds.) (1996). Understanding Risk: Informing Decisions in a Democratic Society. Washington, DC: National Academy Press.

Sutherland, I. (1968). A Head-Mounted Three-Dimensional Display. Proceedings of the AFIPS Fall Joint Computer Conference, 757-764. Washington, D. C.: Thompson Books. 
Tamborini, R., \& Bowman, N.D. (2010). Presence in Video Games. In Campanella, C. \& D. Skalski (eds.), Immersed in Media: Telepresence in Everyday Life, 87-109. London: Routledge.

Tamborini, R. \& Skalski, P. (2006). The Role of Presence in the Experience of Electronic Games. In P. Vorderer and Bryant, J. (eds.), Playing Video Games: Motives, Responses, and Consequences, pp. 263-281. Abingdon: Routledge Taylor \& Francis Group.

Vorderer, P., Klimmt, C., Ritterfeld, U. (2004). Enjoyment: At the Heart of Media Entertainment. Communication Theory, 14(1), 388-408. https://doi. org/10.1111/j.1468-2885.2004.tboo321.x.

Walther, J.B. (2011). Visual Cues in Computer-Mediated Communication: Sometimes Less is More. In Kappas, A., \& Krämer, N.C. (eds.), Face-to-Face Communication over the Internet, pp. 17-38. Cambridge/New York: Cambridge University Press.

Wirth, W., Hartmann, T., Böcking, S., Vorderer, P., Klimmt, C., \& Schramm, H. (2007). A Process Model of the Formation of Spatial Presence Experiences. Media Psychology, 9(3), 493-525. https://doi.org/10.1080/15213260701283079.

Wise, K., Bolls, P.D., Kim, H., Venkataraman, A., \& Moyer, R. (2008). Enjoyment of Advergames and Brand Attitudes. Journal of Interactive Advertising, 9, 27-36. https://doi.org/10.1080/15252019.2008.10722145.

Witmer, B.G., \& Singer, M.J. (1998). Measuring Presence in Virtual Environments: A Presence Questionnaire. Presence: Teleoperators and Virtual Environments, $7(3)$, 225-240. doi:10.1162/105474698565686.

Wu, C., \& Shaffer, D.R. (1987). Susceptibility to Persuasive Appeals as a Function of Source Credibility and Prior Experience with the Attitude Object. Journal of Personality and Social Psychology, 52(4), 677-688. https://doi. org/10.1037/0022-3514.52.4.677.

\section{About the author}

Sun Joo Ahn is founding director of the Games and Virtual Environments Lab in the Grady College of Journalism and Mass Communication at the University of Georgia. Her research examines how social media, video/ internet games, and immersive virtual environments influence user attitudes and behaviors. She is exploring how digital technology can be used to encourage better food choices, more physical activity, and better STEM (science, technology, engineering, and math) learning in children. 



\section{Part III}

Assessing the Effectiveness of Persuasive Games 



\title{
Introduction to Part III
}

\author{
Jeroen Jansz \& Ruud Jacobs
}

The third part of this volume on persuasive gaming is dedicated to validation, a process that assesses the extent to which a specific game succeeds in realizing the intended outcome. For persuasive games, this outcome by definition consists of a change in attitude and/or behavior. Our notion of validation builds upon the theoretical considerations regarding persuasive gaming as discussed in Part I and the criteria for designing persuasive games that were covered in Part II. The chapters included in Part III show that validation research is a small but promising field within game studies. The chapters also show that validation research employed different methods from the social sciences and humanities to determine the effects and impact of persuasive games.

The tone of Chapter 10- The Present of Persuasion: Escalating Persuasive Game Effects Research' — by Ruud Jacobs and Jeroen Jansz is optimistic: The results of previous laboratory and survey research show that many persuasive games have a small but noticeable influence on how players think about the topic addressed in the game. But not all games have been successful. In some studies, it was established that the games were outperformed by other media such as video or print in realizing the intended persuasive outcome. Jacobs and Jansz argue that validation research must evolve to be able to investigate specific game elements because that would lead to results that can be incorporated in future game design as well as generating a deeper media-psychological understanding of game-based messages.

In Chapter 11, 'Designing for Transfer', Mette Wichmand presents an indepth case study of the World Bank's game Urgent Evoke. The game simulates collaborative efforts to tackle large-scale societal issues. It aims for players to transfer the ideas and experiences of working together within the game to the real-world context, spurring them on to become social innovators after their time with the game. Wichmand focused on the winners and links her results to a discussion of how the understanding of successful transfer strategies can inspire future game designs and strengthen the transfer process.

The persuasive game Against All Odds, developed by the United Nations Refugee Agency (UNHCR), is the topic of Chapter 12. In 'Striving "Against All Odds" to Reduce Prejudice towards Immigrants and Refugees', Chad Wertley and Jordan Soliz report their research on the value of using this game 
to change attitudes toward migrant populations-specifically, refugees. They conducted their research in an intergroup setting and found that the effectiveness of Against All Odds in reducing prejudice was limited, although the results supported the notion that persuasive games as a medium do facilitate attitude change. Wertley and Soliz conclude their chapter by stating that more nuanced research into the affordances of persuasive games is required, in particular about how those affordances can best be implemented to achieve the desired goal.

The final chapter in Part III is by Yu-Hao Lee and his colleagues. In 'Mitigating Bias and Improving Professional Decision-Making through Persuasive Training Games', they focus on using games to train people in high-stakes professions such as law enforcement, intelligence analysis, and medicine. They designed three persuasive games that aimed to help professionals to recognize their cognitive biases, improve their knowledge about decision-making biases, and learn ways of mitigating bias. The games consistently outperformed the more static instructional videos in terms of learning, enjoyment, motivation, and engagement. Of particular interest, the bias-mitigation effects of these persuasive games lasted longer than the comparison instructional videos.

The chapters included in this final part of the volume attest to the fact that persuasive game validation research is still developing. We hope this collection helps to highlight the value of bringing together researchers from different topic areas to elevate validation efforts and fortify the medium's recognition as persuasive communication. 


\title{
10. The Present of Persuasion: Escalating Research into Persuasive Game Effects
}

\author{
Ruud Jacobs \& Jeroen Jansz
}

\begin{abstract}
The process of validating persuasive games involves demonstrating that such games are changing or reinforcing specific sets of attitudes in their players. The first wave of validation efforts consisted of simple effect studies in which a full game was compared to other persuasive media or straightforward control conditions. While this led to the conclusion that some persuasive games did indeed 'work', it did not afford generalizations on the viability of gaming as a persuasive medium. We describe these first efforts before showing how subsequent studies are evolving from determining the effects of individual games to testing player-oriented experiential models accounting for multiple persuasive mechanisms. Our conclusions draw on psychological and media-psychological theories of persuasion to offer a roadmap to validating persuasive games.
\end{abstract}

Keywords: validation; effects; attitudes; persuasion; players

Many types of persuasive games have been discussed in the chapters of this volume. Some of these digital games are small and text-based and played in a browser window, while others are multisensory immersive simulations designed to envelop the player completely. Despite their differences, they share a common goal of intending to change attitudes in players: The idea behind their development has been to affect players' beliefs, opinions, and knowledge on topics that are separate from the game itself(De la Hera, 2017; Jacobs, 2016). Naturally, this has led to research (e.g., Gerling, Mandryk, Birk, Miller, \& Orji, 2014; Gustafsson, Bång, \& Svahn, 2009; Peng, Lee, \& Heeter, 2010; Ruggiero, 2015) aiming to establish if these games actually achieve this goal: Do persuasive games really change players' attitudes? Results so far

Hera, T. Dela, J. Jansz, J. Raessens, B. Schouten, Persuasive Gaming in Context. Amsterdam: Amsterdam University Press, 2021 DOI 10.5117/9789463728805_CH10 
bode well for the medium; with a few exceptions, many of the persuasive games that have been tested have small but noticeable influences on how players think about an assortment of topics. These changes are mostly tested in the short term (e.g., Peng et al., 2010), showing that players think differently directly after playing than they did before doing so, although some investigators also examined changes in the longer term (e.g., Ruggiero, 2015). Despite the promising start, there is a long way to go before the viability of games as a persuasive medium can be established conclusively. In this chapter, we will summarize the advances made in persuasive game effect studies thus far and describe the direction that research is - and should be-heading in the near future. We will start by introducing the concept of an effect study and how this has been applied to persuasive games.

\section{Beggars can be choosers: total effects of persuasive games}

Digital games are different from books in terms of how they are perceived by the general public. As an abstract concept, a book is thought to stimulate the imagination, improve language skills, or even support coping in times of tragedy (Koopman, 2011). Digital games, however, are generally seen as playful devices offering nothing but entertainment. What is more, digital entertainment games have been accused of making their players violent (Ivory \& Kalyanaraman, 2009), presumably in the same way that television was thought to 'rot the brain'. Currently, this negative perception is weakening, probably because of the growing familiarity with different game genres, including non-violent ones, and the penetration of all kinds of digital games in all levels of society (Bourgonjon, Valcke, Soetaert, de Wever, \& Schellens, 2011; Kneer, Jacobs, \& Ferguson, 2018). Thankfully, interest in the effects of games beyond their promotion of violent behaviors has grown steadily over the years, and a budding knowledge base has been formed for effects ranging from positive and general (Granic, Lobel, \& Engels, 2013) to title- and topic-specific learning outcomes (DeSmet et al., 2014).

Despite being about as old as games themselves, persuasive games have until recently received comparatively little attention. Indeed, many of the classic games of yesteryear have messages that are simply not acknowledged by many of their players (Flanagan, 2009). This is perhaps why testing the effects of such games is a relatively new phenomenon; the first published forays into the attitudinal effects of persuasive games are less than a decade old at the time of writing (Alhabash \& Wise, 2012; Barthel, 2013; Peng et al., 2010, among others). These studies attempt to answer a very basic question 
that spawned from the early resentment and trivialization of digital games: Do persuasive games even work? As newcomers to (mediated) interventions that require complex design decisions and can, in some instances, demand exponential budgets, persuasive games, much like other serious games, needed to be proven to work to be taken seriously (Siriaraya, Visch, Vermeeren, \& Bas, 2018). The first wave of effect studies therefore had the mission to identify the effects of games in toto, often by running participants through the game as a whole or several key moments in it (Van 't Riet, Meeuwes, Van der Voorden, \& Jansz, 2018), or by letting them play for as long as they wanted (Jacobs, 2016). In such studies, the games were off-the-shelf or ready-made experiences, such as Darfur is Dying (Ruiz, York, Stein, Keating, \& Santiago, 2006) and My Cotton Picking Life (Rawlings, 2012). The results of these studies indicate that, in most cases, persuasive games really do affect the way their players think, both in the short term (Kampf \& Cuhadar, 2015; Peng et al., 2010) and weeks after play has finished (DeSmet et al., 2018; Ruggiero, 2015). As with any kind of mediated intervention, other studies reported a lack of effects or effects confined to specific game elements (Soekarjo \& Van Oostendorp, 2015; Van 't Riet et al., 2018).

The researchers studying whether persuasive games 'worked' based their claims on empirical, quantitative testing of the attitudes held by players. In most cases, this involved asking players to agree or disagree with written statements that indicate a specific stance toward an issue. This kind of self-reported attitude testing is highly flexible when compared to more objective measures like the implicit association test (used by Gerling, Birk, \& Mandryk, 2014; Gutierrez et al., 2014; and Shaw, Crosby, \& Porter, 2014), as it allows the researcher to match the measurement to the specific topic. Persuasive games deal with diverse topics and even vary greatly in what they focus on within a topic area (Jacobs, Jansz, \& De la Hera, 2017); indeed, in all but a few cases, it is not particularly fruitful to try to gauge the effects of games with more general measurements. In cases where there is not already a testable criterion or an attitudinal benchmark that the game is meant to achieve, we encourage researchers planning to test a game to determine what its attitude goal state is (Jacobs, 2017). The attitude goal state is linked to the game's design, describing the attitudes that a game is built to convey to its players. While a game could theoretically have widespread attitudinal and broader effects, a persuasive game can only be validated in terms of how its intended attitudinal effects are actually realized in players. If the attitude goal state cannot be obtained from the game's designers, it can be reverse-engineered through play-tests. This involves exploring the game, analyzing what it shows and tells the player, and also determining 
the kind of decisions it enables the player to make and how it responds to those decisions. In addition, expert testing with designers can contribute to understanding some of the more complex features of the game's design, although such testing is not necessary to approximate the attitude goal state in relation to its realization in actual play.

After the attitude goal state is established, researchers can try to match existing attitude scales to this state, as was done with the 'Attitudes toward the Homeless Inventory' in the game Spent (Ruggiero, 2015) and the 'Justification of Verbal and Coercive Tactics' scale (Slep, Cascardi, Avery-Leaf, \& O'Leary, 2001) for games on dating violence in our own work (Jacobs, 2017, Chapter 6). In many other cases, however, the game's message is idiosyncratic, making it difficult for it to be reliably covered by any one attitude scale. In cases like this, it is best to design a proprietary scale with several subscales, deriving items from other validated scales whenever possible. In the case of My Cotton Picking Life (Rawlings, 2012), the attitude goal state consisted of attitudes with regard to modern-day slavery and how players can be empowered to curb it, as well as harsh working conditions among cotton pickers in Uzbekistan (Jacobs et al., 2017). Testing the game's effects in comparison to a YouTube video on the same topic, we found that the game and video proffered roughly the same message when it came to slavery and empowerment but that the game was better at conveying the intensity, severity, and endless nature of the actual labor involved in picking cotton. If a unidimensional scale had been used, this effect might have been overlooked. Of course, proprietary scales are generally less reliable than validated scales, which makes it necessary to enforce rigorous standards (Ruggiero, 2015). Scales should be factor-analyzed, tested for reliability, and shared whenever the study is reported to enable other researchers to replicate the study or simply to see whether the scale does indeed match the attitude goal state.

The conscious and targeted application of games as persuasive experiences is part of an emerging tradition of using digital games for purposes beyond entertainment (De la Hera, 2017). Given the novelty of this approach, many persuasive games compete with interventions that make use of older, more established media. This can be seen in the design of effect studies: persuasive games are often compared with persuasive texts (Gutierrez et al., 2014; Peng et al., 2010; Ruggiero, 2015; Soekarjo \& van Oostendorp, 2015), videos (Jacobs, 2016), or a combination of these (Steinemann, Mekler, \& Opwis, 2015; Van 't Riet et al., 2018). Studies that replace the traditional no-treatment control group with this kind of alternative stimuli can only determine the discriminant validity of such games, meaning that they can only prove 
whether a game works better than other media rather than establishing the absolute effect of a game by itself. Moreover, the aforementioned unique messages that are expressed through the full experience of a persuasive game are more often than not different from those found in other media, warranting a careful matching of competing stimuli for any effect study (Jacobs, 2016). Studies with a no-treatment control (e.g., Barthel, 2013; DeSmet et al., 2018), or those that employ both separately (Jacobs, 2017, Chapter 6; Ruggiero, 2015) are arguably better suited to answering the question of whether persuasive games work, while studies with alternative interventions present a more realistic picture of the added benefit that using a game might have in a world where individuals are flooded with persuasive attempts daily throughout their lives.

The results of studies into games as complete experiences have so far yielded important insights, though their primary contribution has been to provide varying levels of proof that games do indeed work as tools of deliberate persuasion. Apart from supporting conclusions to use or not use games in specific campaigns, this research serves the purposes of those making games in general by conferring legitimacy to the medium as a whole. Unfortunately, basic effect studies do not necessarily help designers make more persuasive games - results on a game as a whole are not generalizable to other games with different designs, nor do they allow researchers to find the underlying mechanisms of the persuasive impact of games. This is why researchers have started identifying elements within games that determine their impact.

\section{Inspecting the gift horse: comparing the effects of persuasive games}

Knowing that some games persuade while others do not is not productive for individual designers or other stakeholders of games that have not yet been validated. As demonstrated by Van 't Riet et al. (2018) and in Chapter 12 of this volume, Against All Odds (UNHCR, 2006) failed to produce lasting change when compared to reading a text or watching a video. This could mean any of three things: 1) that the game was not persuasive because of flaws in its design; 2) that each of the studies performed only let participants play small parts of the game and so the persuasive effect was diluted; or 3) that the players in different settings did not appreciate the use of games to discuss this highly contentious issue. While there will always be confounds even in the most rigorous designs, meaning that the second and third possibilities 
can never be wholly discounted, a few researchers have worked to determine the first possibility more conclusively by comparing different versions of a persuasive game.

One of the more comprehensively explored avenues within differentiated designs of persuasive games was charted by Rita Orji and colleagues (Orji, Mandryk, Vassileva, \& Gerling, 2013; Orji, Nacke, \& Di Marco, 2017; Orji, Vassileva, \& Mandryk, 2014). Espousing a view of human-centered design, she proposed matching the gameplay and experience of persuasive games to the personalities of their players; for example, players scoring high on an extraversion scale would enjoy games that allow them to personalize their experience more than those scoring high on a neuroticism scale (Orji et al., 2017). In cases where a target group shares a definable personality trait, this kind of knowledge can be a boon. However, most persuasive games are meant to communicate an issue to an audience that is as wide as possible (i.e., with varied personalities and interests). In this case, the personality-oriented approach might end up needlessly constricting the player base. Moreover, the evidence provided in these studies is based on self-reported perceptions of the impact of persuasive mechanisms. The insights delivered by Orji et al. (2017) are therefore most interesting for designers hoping to cast a wide net, appealing to the largest number of players, although more tangible evidence is needed to determine which kind of game is most effective. Moreover, any relationships need to be established on a causal level. Rogers and Weber's investigation (2018) of the effect of controversy in persuasive games on a willingness to donate, for example, could not determine whether the controversial aspects of the games played were actually causing changes in behavior.

We applied a model to categorize the persuasive mechanisms employed in games (De la Hera, 2019) to a selection of freely available online persuasive games (Jacobs et al., 2017). The findings helped constrain the search for which broad-stroke design decisions we should compare; although persuasive games are unique and deal with a wide range of issues in their own unique ways, they tend to rely on narratives or procedural rhetoric (Bogost, 2007; see also this volume) to bear most — though certainly not all—of the persuasive heft. Following up on this distinction, we sought out two persuasive games that offered the same message but focused either on narrative persuasion or procedural rhetoric (Jacobs, 2017, Chapter 6). The two games were Power and Control (Sain, 2011) and Another Chance (Another Kind, 2015), which were published by Jennifer Ann's Group (Jennifer Ann's Group, n.d.) to combat teen dating violence. Both games were judged on the same criteria and so dealt with the same topic. Whereas Power and Control focused on 
reflecting the issue of abuse in relationships through its approach-and-avoid gameplay, Another Chance played much like a 16-bit adventure game and instead tried to persuade players with its narrative of a girl realizing that her boyfriend's behaviors are abusive. Interestingly, despite the fact that Another Chance took around three times as long to play as Power and Control, the two games were equally effective in changing attitudes when compared to a control game. This study was not an attempt to control for every aspect of a game but a first comparison of different games with the same message. Supported by scales that measured whether players felt persuaded by the storyline and whether the gameplay reflected the real-world situation it depicted-a linking process termed cognitive identification by Williams and Williams (2007) —we concluded that, when properly implemented, narrative and procedural forms of persuasion might end up having effects that are so similar as to be considered equal.

More direct effect studies that tweak certain aspects of a game while controlling all others have been published, although they are rare. In these cases, researchers would need to either cooperate with designers who are working iteratively (as explained by Van Broeckhoven, Vlieghe, \& De Troyer, 2015) or simply design and manipulate the games themselves. One of the first instances of the latter was the game Birthday Party (Gerling, Mandryk, et al., 2014), which was developed to change attitudes toward people with disabilities. By asking participants to play with either a special wheelchairbased control system or a traditional controller, Gerling and colleagues found that the way players interact with a game-outside of the gameplay itself-can moderate the game's attitudinal effects. A very recent study examined whether news games - those that are concerned with a current event that often have strong persuasive elements - would benefit from a certain graphical style over others ( $\mathrm{Lin} \& \mathrm{Wu}, 2018)$. When comparing a more cartoonish presentation to a style that is considered to be more adult, no differences emerged in the knowledge gained by participants. However, the more professional presentation style led to a greater appreciation of the game, which in turn affected behavior (in particular, donation behavior). Clearly, individual aspects of a game experience can influence elements of the experience in such a way as to add to or detract from a game's persuasiveness.

One of the principal factors of persuasive games that is perhaps most worthy of study is the procedural rhetoric that they offer (Bogost, 2007). This concept is, however, hard to conceptualize, let alone operationalize. As it amounts to letting the system and rule-sets in a game speak for themselves, procedural rhetoric is dependent on games' interactivity. Seeing interactivity as vital to games, Peng et al. (2010) manipulated it in their early study. 
They found that playing the game Darfur is Dying - as opposed to simply watching pre-recorded footage of it-led players to be more willing to help refugees. Surprisingly, though, they also found that levels of enjoyment were greater for non-interactive conditions than interactive ones. Another study on the same game by Steinemann et al. (2015) also found a difference between interactive and non-interactive conditions, with the interactivity making players more appreciative of the game's story. Clearly, then, the manipulation of interactivity has knock-on effects on players' experiences apart from procedural rhetoric.

Working with game researcher Stefan Werning, we sought to alter the procedural rhetoric of a game without affecting its interactivity (Jacobs, 2017, Chapter 7 ). This was done in a way that was similar to how more traditional persuasion research is performed, namely by presenting weaker or stronger arguments. The game My Cotton Picking Life (Rawlings, 2012) amounts to a scripted representation of picking cotton in a field as a boring, endless task. It was redesigned to allow players to finish their daily picking quota in a manner of seconds. Care was taken to hold the game's story constant - the game would just go to a new day once players finished their quota-and retain its original atmosphere, visuals, and sense of tactility. The change in feedback whenever the pick buttons were clicked was, in this sense, solely responsible for the strongly diminished attitude change among players of the new 'easy' mode. Since procedural rhetoric is the main element that sets games apart from other types of intervention, this conclusion can be quite informative for anyone planning to develop their intervention as a persuasive game. These experiences persuade players in a way that no other medium or interpersonal interaction does. At the same time, much work is left to be done before we can say that procedural rhetoric is stronger than other types of persuasion, as such a conclusion would imply a systematic comparison of different rhetorical strategies within the same persuasive game.

\section{Tearing open the black box: the mechanics of game-based persuasion}

We now know that games do have a persuasive impact in plenty of instances, and some work has been published to determine what elements in a game's design can cause it to be effective. However, persuasive games are such rich, multimodal experiences that theoretical arguments for how these games work on an individual level are quite scarce. Thankfully, 
with the benefit of an established field of persuasion research (Perloff, 2014), investigators did not need to reinvent the wheel when it came to predicting the mechanisms of attitude change from persuasive games. The theory of planned behavior (Ajzen, 1991) made it possible to focus on attitude change over behavioral change, which is comparatively harder to gauge (DeSmet et al., 2018). More specific theories, such as stages of change (Slater, 1999), identification (Walz, 2004), and perspective taking (Gutierrez et al., 2014), have already been applied to game-based interventions. However, the current body of research on the effects of persuasive games is lacking support from psychological mechanisms, as many of the attempts to apply such mechanisms to games thus far have not involved adapting them to the unique experience of playing a game. So, even though games do indeed have narratives, they are not always linear storylines with spots of gameplay in between. Their interactive nature often causes these narratives to have to be able to bend to the will of the player, leading to different outcomes. In Depression Quest (Quinn, 2013), the narrative is even intertwined with the procedural rhetoric, as narrative options are diminished as the protagonist's depressive state worsens. Steinemann et al. (2017) have shown the importance of linking narratives to interactivity, but their study results in new questions such as: How would players react when their agency in narratives is threatened? Or: How would they respond to being presented with a situation that has no positive endings (see also Ruggiero \& Becker, 2015)?

Researchers need to identify how advances in game studies can help connect the effects of games with theories of attitude change. In our research, we have attempted to link the elaboration likelihood model (Petty \& Cacioppo, 1986) - which describes how our ability and motivation to consider a persuasive message can influence how we are persuaded by arguments and other elements of a message - to the changes in procedural rhetoric described in the previous section (Jacobs, 2017, Chapter 7). Aside from changing the gameplay, two further conditions were created. In the first, players were exposed to much the same version of My Cotton Picking Life as originally created (Rawlings, 2012), although diegetic music was added in the form of a radio playing a local song. In the second condition, several additional aural and visual stimuli were added, which were carefully selected to not interfere with the messaging of the game: flocks of birds flew over the play area; white noise was overlaid on the game screen; the on-screen buttons were animated; and the music was played at a higher volume with more distortion. These changes were meant to increase the players' cognitive load (Vyvey \& Núñez Castellar, 2016) which, according 
to the elaboration likelihood model, should have caused them to be able to focus less on the central arguments presented by the game. This study was intended to shed light on how players elaborate on non-verbal, gameplaybased messages, hypothesizing that this type of argument is as reliant on elaboration as traditional, verbally delivered arguments. Unfortunately, the manipulation did not make the players report higher cognitive loads. This meant that, in our case, there were virtually no differences between conditions with a low and high cognitive load. We hope that this study can be replicated with a different game, while improving on the manipulation of the cognitive load. The possibilities for such a manipulation abound (e.g., presenting time-limits, increasing the difficulty, further increasing the visual and aural effects, forcing players to do a secondary task at the same time), although each manipulation can introduce confounds to the concurrent procedural rhetoric manipulation. Meeting this challenge and finding evidence to support either a traditional mechanism of elaboration or even that the procedural rhetoric is absorbed on a different level than the verbal rhetoric would certainly underline the validity of games as a persuasive medium.

There are more open questions when it comes to the mechanisms of persuasive games' effects. Principally, we believe that there is a near-universal misconception, both in industry and academia, that the most important thing for any serious game to be is fun to play (Jacobs, 2017, Chapter 8). This is a non-sequitur that does not exist in any other medium and is likely the result of the position that games are for children — and worse, for children who can only be tempted with the promise of vacuous entertainment. Seen in this way, the primacy of enjoyment is a vestigial element in the perception of games - much like external perceptions of why we play violent games (Kneer et al., 2018) — that is set to wane over time. In our research, we found that there are other elements to the experience of a persuasive game that are measurable and offer far better predictive validity of the appreciation of these games. Just as we would not watch Schindler's List (Spielberg, 1993) to have a fun time, we would not experience abuse in a game by the Jennifer Ann's Group (Crecente, 2014) with the expectation that the gameplay would make us smile. Instead, these experiences offer us the chance to experience meta-emotions like eudaimonia and promote our intellectual, virtuous growth as individuals (Oliver \& Bartsch, 2010). Translating this into a short scale for persuasive games, we found that eudaimonic appreciation was related to the attitudinal effects of different persuasive games, while the relationship with hedonic enjoyment was much more sporadic (Jacobs, 2017, Chapter 8). 


\section{Conclusion}

Over the past few years, researchers have taken the first steps toward validating persuasive games. This chapter aimed to present the results of this emerging tradition. It also showed that much is still unknown about how players relate to persuasive games, why they play them, and how such games change players' attitudes. Researchers need to escalate their investigations beyond direct comparisons of full persuasive games to control stimuli. This is a daunting task, as persuasive games offer an even wider variety than non-interactive audiovisual stimuli, let alone text. There is a deep psychological entanglement of the players, the game, and the context in which they play that is rife with insights waiting to be uncovered. Future investigations should include personality characteristics that directly relate to persuasion — such as the need for cognition (Cacioppo \& Petty, 1982) — and link them to the effects of different persuasive dimensions (De la Hera, 2015) as well as where and in what company a game is played (De Grove, van Looy, Neys, \& Jansz, 2012). The full picture that such studies would generate can help tie models of persuasion into what makes games unique. Most importantly, however, it can help game designers make more involving games that allow players to reach their own well-supported conclusions on any relevant issue.

\section{References}

Ajzen, I. (1991). The Theory of Planned Behavior. Organizational Behavior and Human Decision Processes, 5o(2), 179-211. https://doi.org/10.1016/0749-5978(91)90020-T.

Alhabash, S., \& Wise, K. (2012). Peacemaker: Changing Students' Attitudes Toward Palestinians and Israelis Through Video Game Play. International Journal of Communication, 6, 356-380. Retreived 9 June 2018 from https://ijoc.org/index. php/ijoc/article/view/1056.

Another Kind. (2015). Another Chance [Online Game].Jennifer Ann's Group. Atlanta, GA. Retrieved 4 March 2018 from https://jagga.me/anotherchance.

Barthel, M.L. (2013). President for a Day: Video Games as Youth Civic Education. Information, Communication \& Society, 16(1), 28-42. https://doi.org/10.1080/136 9118X.2011.627176.

Bogost, I. (2007). Persuasive Games. Cambridge, MA: MIT Press.

Bourgonjon, J., Valcke, M., Soetaert, R., De Wever, B., \& Schellens, T. (2011). Parental Acceptance of Digital Game-Based Learning. Computers \& Education, 57(1), 1434-1444. https://doi.org/10.1016/j.compedu.2010.12.012. 
Cacioppo, J.T., \& Petty, R.E. (1982). The Need for Cognition. Journal of Personality and Social Psychology, 42(1), 116-131. https://doi.org/10.1037/0022-3514-42.1.116.

Crecente, D. (2014). Gaming Against Violence: A Grassroots Approach to Teen Dating Violence. Games for Health Journal, 3(4), 198-201. https://doi.org/doi:10.1089/ g4h.2014.0010.

De Grove, F., Van Looy, J., Neys, J., \& Jansz, J. (2012). Playing in School or at Home? An Exploration of the Effects of Context on Educational Game Experience. Electronic Journal of E-Learning, $10(2), 199-208$. Retreived 5June 2018 from https:// eric.ed.gov/?id=EJ985422.

De la Hera, T. (2019). Digital Games and the Advertising Landscape. Amsterdam: Amsterdam University Press.

- (2015). A Theoretical Model for the Study of Persuasive Communication through Digital Games. In J.M. Parreno, C.R. Mafe, \& L. Scribner (eds.), Engaging Consumers through Branded Entertainment and Convergent Media, pp. 74-88. Hershey, Pennsylvania: IGI Global. https://doi.org/10.4018/978-1-4666-8342-6.

—. (2017). Persuasive Gaming: Identifying the Different Types Of Persuasion Through Games. International Journal of Serious Games, 4(1), 31-39. https://doi. org/10.17083/ijsg.v4i1.140.

DeSmet, A., et al. (2018). The Efficacy of the Friendly Attac Serious Digital Game to Promote Prosocial Bystander Behavior in Cyberbullying Among Young Adolescents: A Cluster-Randomized Controlled Trial. Computers in Human Behavior, 78, 336-347. https://doi.org/10.1016/j.chb.2017.10.011.

. (2014). A Meta-Analysis of Serious Digital Games for Healthy Lifestyle Promotion. Preventive Medicine, 69, 95-107. https://doi.org/10.1016/j. ypmed.2014.08.026.

Flanagan, M. (2009). Critical Play. Radical Game Design. Cambridge, MA: MIT Press. Gerling, K.M., Birk, M.V., \& Mandryk, R.L. (2014). Combining Explicit and Implicit Measures to Study the Effects of Persuasive Games. In: CHI 2014 Workshop "Games User Research on Mixed Methods and Reporting Results”, 17 April 2014, Toronto, Canada. Retreived 7 June 2018 from http://eprints.lincoln.ac.uk/id/eprint/13693/. — , Mandryk, R.L., Birk, M.V., Miller, M., \& Orji, R. (2014). The Effects of Embodied Persuasive Games on Player Attitudes Toward People Using Wheelchairs. Proceedings of the $32 n d$ Annual ACM Conference on Human Factors in Computing Systems - CHI'14, pp. 3413-3422. https://doi.org/10.1145/2556288.2556962.

Granic, I., Lobel, A., \& Engels, R.C.M.E. (2013). The Benefits of Playing Video Games. American Psychologist, 69(1), 66-78. https://doi.org/10.1037/aoo34857.

Gustafsson, A., Bång, M., \& Svahn, M. (2009). Power Explorer: A Casual Game Style for Encouraging Long Term Behavior Change Among Teenagers. ACE 'og Proceedings of the International Conference on Advances in Computer Entertainment Technology, pp. 182-189. https://doi.org/10.1145/1690388.1690419. 
Gutierrez, B., Kaatz, A., Chu, S., Ramirez, D., Samson-Samuel, C., \& Carnes, M. (2014). "Fair Play": A Videogame Designed to Address Implicit Race Bias Through Active Perspective Taking. Games for Health Journal, 3(6), 371-378. https://doi. org/10.1089/g4h.2013.0071.

Ivory, J.D., \& Kalyanaraman, S. (2009). Video Games Make People Violent-Well, Maybe Not That Game: Effects of Content and Person Abstraction on Perceptions of Violent Video Games' Effects and Support of Censorship. Communication Reports, 22(1), 1-12. https://doi.org/10.1080/08934210902798536.

Jacobs, R.S. (2016). Play to Win Over: Effects of Persuasive Games. Psychology of Popular Media Culture. https://doi.org/10.1037/ppmoooo124.

_. (2017). Playing to Win Over: Validating Persuasive Games [PhD Thesis]. ERMeCC-Erasmus Research Center for Media Communication and Culture. Retrieved from http://hdl.handle.net/1765/102769.

— Jansz, J., \& De la Hera, T. (2017). The Key Features of Persuasive Games: A Model and Case Analysis. In R. Kowert \& T. Quandt (eds.), New Perspectives on the Social Aspects of Digital Gaming: Multiplayer 2, pp. 153-171. Oxford: Routledge. Jennifer Ann's Group. (n.d.). JAG Ga.me: Emotional Health And Wellness Games For Teens, Parents, And Teachers. Retrieved 5 May 2018 from https://jagga.me/. Kampf, R., \& Cuhadar, E. (2015). Do Computer Games Enhance Learning About Conflicts? A Cross-National Inquiry into Proximate and Distant Scenarios in Global Conflicts. Computers in Human Behavior, 52 (Supplement C), 541-549. https://doi.org/10.1016/j.chb.2014.08.008.

Kneer, J., Jacobs, R., \& Ferguson, C.J. (2018). You Could Have Just Asked: The Perception of Motivations to Play Violent Video Games. Studies in Media and Communication, 6(2), 1. https://doi.org/10.11114/smc.v6i2.3389.

Koopman, E. (2011). Predictors of Insight And Catharsis Among Readers Who Use Literature as a Coping Strategy. Scientific Study of Literature, 1(2), 241-259. https:// doi.org/10.1075/ssol.1.2.04koo.

Lin, J.-H., \& Wu, D.-Y. (2018). Are Newsgames Children's Games? Examining The Effects of Graphic Realism and Issue Proximity on Players' Willingness to Forward and Donate and The Underlying Mechanisms. Submitted for publication.

Oliver, M.B., \& Bartsch, A. (2010). Appreciation as Audience Response: Exploring Entertainment Gratifications Beyond Hedonism. Human Communication Research, 36(1), 53-81. https://doi.org/10.1111/j.1468-2958.2009.01368.x.

Orji, R., Mandryk, R.L., Vassileva, J., \& Gerling, K.M. (2013). Tailoring Persuasive Health Games to Gamer Type. Proceedings of the SIGCHI Conference on Human Factors in Computing Systems - CHI '13, pp. 2467-2476. https://doi. org/10.1145/2470654.2481341.

— Nacke, L.E., \& Di Marco, C. (2017). Towards Personality-Driven Persuasive Health Games and Gamified Systems. In Proceedings of the 2017 CHI Conference 
on Human Factors in Computing Systems - CHI '17, pp. 1015-1027. New York, NY: ACM Press. https://doi.org/10.1145/3025453.3025577.

__— Vassileva, J., \& Mandryk, R.L. (2014). Modeling the Efficacy of Persuasive Strategies for Different Gamer Types in Serious Games for Health. User Modeling and User-Adapted Interaction, 24(5), 453-498. https://doi.org/10.1007/ s11257-014-9149-8.

Peng, W., Lee, M., \& Heeter, C. (2010). The Effects of a Serious Game on Role-Taking and Willingness to Help.Journal of Communication, 6o(4), 723-742. https://doi. org/10.1111/j.1460-2466.2010.01511.x.

Perloff, R. (2014). The Dynamics of Persuasion: Communication and Attitudes in the Twenty-First Century (5th ed.). New York, NY: Routledge.

Petty, R.E., \& Cacioppo, J.T. (1986). The Elaboration Likelihood Model of Persuasion. Advances in Experimental Social Psychology, 19, 123-205. https://doi.org/10.1016/ Soo65-2601(08)60214-2.

Quinn, Z. (2013). Depression Quest [Online Game]. Retrieved 19 July 2018, from http://www.depressionquest.com/.

Rawlings, T. (2012). My Cotton Picking Life [Online Game]. Bristol, UK: GameTheNews. Retrieved from http:/gamethenews.net/index.php/my-cotton-picking-life/. Rogers, K., \& Weber, M. (2018). Shock Tactics: Perceived Controversy in Molleindustria Persuasive Games. In Persuasive 2018: Persuasive Technology, pp. 193-199. https://doi.org/10.1007/978-3-319-78978-1_16.

Ruggiero, D. (2015). The Effect of Playing a Persuasive Game on Attitude and Affective Learning. Computers in Human Behavior, 45, 213-221. https://doi.org/10.1016/j. chb.2014.11.062.

—, \& Becker, K. (2015). Games You Can't Win. The Computer Games Journal, 4(3-4), 169-186. https://doi.org/10.1007/s40869-015-0013-9.

Ruiz, S., York, A., Stein, M., Keating, N., \& Santiago, K. (2006). Darfur Is Dying [Online Game]. New York, USA: MTV Networks. Retrieved from http://www. darfurisdying.com/.

Sain, J. (2011). Power and Control [Online Game].Jennifer Ann's Group. Atlanta, GA. Retrieved from http://jenniferann.org/2011-game-third-place.htm.

Shaw, J., Crosby, K., \& Porter, S. (2014). The Impact of a Video Game on Criminal Thinking: Implicit and Explicit Measures. Simulation \& Gaming, 45(6), $786-804$. https://doi.org/10.1177/1046878115574018.

Siriaraya, P., Visch, V., Vermeeren, A., \& Bas, M. (2018). A Cookbook Method for Persuasive Game Design. International Journal of Serious Games, 5(1). https:// doi.org/10.17083/ijsg.v5i1.159.

Slater, M.D. (1999). Integrating Application of Media Effects, Persuasion, and Behavior Change Theories to Communication Campaigns: A Stages-Of-Change 
Framework. Health Communication, (November 2013), 37-41. https://doi. org/10.1207/S15327027HC1104.

Slep, A.M., Cascardi, M., Avery-Leaf, S., \& O’Leary, K.D. (2001). Two New Measures of Attitudes About the Acceptability of Teen Dating Aggression. Psychological Assessment, 13(3), 306-318. https://doi.org/10.1037/1040-3590.13.3.306.

Soekarjo, M. \& van Oostendorp, H. (2015). Measuring Effectiveness of Persuasive Games Using an Informative Control Condition. International Journal of Serious Games, 2(2), 37-56. https://doi.org/10.17083/ijsg.v2i2.74.

Spielberg, S. (1993). Schindler's List. United States: Universal Pictures.

Steinemann, S.T., Iten, G.H., Opwis, K., Forde, S.F., Frasseck, L., \& Mekler, E.D. (2017). Interactive Narratives Affecting Social Change: A Closer Look at the Relationship Between Interactivity and Prosocial Behavior. Journal of Media Psychology, 29(1), 54-66. https://doi.org/10.1027/1864-1105/a000211.

Steinemann, S.T., Mekler, E.D., \& Opwis, K. (2015). Increasing Donating Behavior Through a Game for Change: The Role of Interactivity and Appreciation. In Proceedings of the 2015 Annual Symposium on Computer-Human Interaction in Play. New York, NY: ACM Press. https://doi.org/10.1145/2793107.2793125.

UNHCR. (2006). Against All Odds. Retrieved from http://www.playagainstallodds. ca/.

Van 't Riet, J., Meeuwes, A.C., Van der Voorden, L., \& Jansz, J. (2018). Investigating the Effects of a Persuasive Digital Game on Immersion, Identification, and Willingness to Help. Basic and Applied Social Psychology, 1-15. https://doi.org/1 0.1080/01973533.2018.1459301.

Van Broeckhoven, F., Vlieghe, J., \& De Troyer, O. (2015). Mapping between Pedagogical Design Strategies and Serious Game Narratives. In 2015 th International Conference on Games and Virtual Worlds for Serious Applications (VS-Games), pp. 1-8. IEEE. https://doi.org/10.1109/VS-GAMES.2015.7295780.

Vyvey, T., \& Núñez Castellar, E.P. (2016). Loaded with Fun? An Experimental Study into Enjoyment And Cognitive Load as Determinants of In-Game Advertising Retention. Retrieved 15 March 2018 from https://biblio.ugent.be/ publication/8060307.

Walz, S.P. (2004). Delightful Identification \& Persuasion: Toward an Analytical and Applied Rhetoric of Digital Games. Works and Days, 22(43/44), 185-200. Retrieved 1 July 2018 from http://worksanddays.net/2004/File21.Walz_File21.Walz.pdf.

Williams, R.H., \& Williams, A.J. (2007). In Pursuit of Peace: Attitudinal and Behavioral Change with Simulations and Multiple Identification Theory. Simulation \& Gaming, 38(4), 453-471. https://doi.org/10.1177/1046878107300675. 


\section{About the authors}

Ruud Jacobs researched the validation of persuasive games at the Erasmus University Rotterdam as part of the research project Persuasive Gaming. From Theory-Based Design to Validation and Back (NWO). He now works as an Assistant Professor of communication and technology at the University of Twente, investigating why people play serious games.

Jeroen Jansz is Full Professor of Communication and Media at Erasmus University Rotterdam. He holds the Chair of Communication and Media in the Department of Media \& Communication. His research aims to understand and explain the changing relations between media consumers and media producers with a specific focus on digital gaming. 


\title{
11. Designing for Transfer
}

\author{
Mette Wichmand
}

\begin{abstract}
How can a game design support the transfer of human resources from an in-game world to the physical world? A possible answer can be found in the in-depth case study of the World Bank's game Urgent Evoke presented in this chapter. Urgent Evoke is a game designed to empower players to become post-game social innovators, and this chapter shows how the winners' interaction with and sense-making of the game enabled a successful transfer to and implementation of their ideas in the physical world. The analysis is based on activity theory and leads to a discussion of how an understanding of the successful transfer strategies of the winners can inspire future game designs and strengthen the transfer process.
\end{abstract}

Keywords: game design; transfer; empowerment; social innovation; activity theory

\section{Background}

The gaming industry has diversified its means of expression over the years. Today, digital games can be played alone; with a friend; or with thousands of strangers; simultaneously or not; via a computer, tablet, or mobile phone; at home or on the go. A game can be filled with words or may offer a simple ${ }_{2} \mathrm{D}$ interface or a $3 \mathrm{D}$ immersive virtual world. It can let you play with reality as you know it or take you to new or historical environments or even to a fantasy world for you to explore. It can take a minute to play or as many hours and days as you choose. The possibilities are therefore endless, and this has led to games being produced, used, and seen not only as tools for entertainment but also as persuasive mediums that 'like the protected books, plays, and movies that preceded them [...] communicate ideas - and even social messages - through many familiar devices (such as characters,

Hera, T. Dela, J. Jansz, J. Raessens, B. Schouten, Persuasive Gaming in Context. Amsterdam: Amsterdam University Press, 2021 DOI 10.5117/9789463728805_CH11 
dialogue, plot, and music) and through features distinctive to the medium (such as the player's interaction with the virtual world)' (Brown, Governor of California, et al. v. Entertainment Merchants Association et al., Supreme Court of the United States, 27 June 2011).

The World Bank's social innovation game Urgent Evoke is a state-of-the-art example of a persuasive game. What's interesting about a game like this is that it not only aims to communicate ideas and social messages, it also makes the claim that playing the game will enable players to transfer the knowledge, ideas, and other resources found and constructed in the game to the physical world, enabling them to become post-game social innovators capable of wrestling with some of the world's most serious problems, e.g., poverty and hunger. If and how this transfer is enabled by the game design and the players' use of the game is the core issue in this chapter.

\section{Urgent Evoke}

Urgent Evoke (UE) is an online social network game that's free to play via a computer, tablet, or smartphone. The game was developed by the World Bank Institute and game designer Jane McGonigal. Its aim, as noted above, is to generate post-game, player-driven social innovation.

UE was played for the first time in 2010 over a ten-week period. The game was open to players of all ages and could be played anywhere in the world. Today, the original game can still be found online but is now only available to high school teachers as an educational tool.

UE revolves around a narrative told in the form of a graphic novel. A new chapter was released each week. The narrative driving the game tells the story of a secret international network of agents with innovative superpowers capable of solving some of the world's most serious problems (hunger, armed conflict, environmental challenges, etc.). Players take on the role of agents in the network and are given a new mission each week. The missions are connected to the serious problems presented in the ten chapters of the graphic novel. The tasks are formulated to help players reflect upon and strengthen their personal capacity as a social innovator.

To win the game, players had to complete ten missions and ten quests and turn in an Evokation. An Evokation is a detailed and innovative plan of how the player will tackle a self-chosen challenge in the physical world after the game has ended. The World Bank evaluated the Evokations, and the plans found to be the most promising were rewarded with a mix of seed money, mentorships by respected social innovators and entrepreneurs, and 
an opportunity for the winners to attract crowd funding via the Global Giving Challenge (www.globalgiving.org/evoke). A subset of winners was also invited to a post-game EVOKE summit held in Washington, DC.

Along with the winners, players who completed all ten missions and quests were recognized for their engagement and received World Bank Institute certificates stating that they are certified social innovators. Their names are mentioned on the UE website as 'Certified EVOKE Social Innovators - Class of 2010'. Players who completed one or more missions and quests were recognized on the UE website as one of 'the EVOKE class of 2010 graduates'.

During the initial ten-week run-time in 2010, UE attracted unprecedented public attention for a serious game: 171,958 different individuals made 286,219 visits to the game; 19,386 people registered as players; 6,618 people completed at least one mission or quest; 142 players completed all ten missions and quests; 73 people submitted Evokations; and 32 Evokations were awarded by the World Bank (Gaible \& Dabla, 2010). The game's intention to empower the players was built into and communicated through all parts of its design.

One of the ways it did this can be found in the designers' blurring of the line between the in-game world and the physical world. UE offered its players a safe, artificial space where the rules of the physical world were, in some ways, vaporized. Inside this 'magic circle' (Huizinga, 1955), it was possible to play the role of a social innovator with superpowers and come up with bold, innovative ideas without being afraid that millions are being lost or people are dying of hunger if your idea turned out to be more creative than sustainable. Yet the magic circle of UE was also designed to give players a sense of reality. Castronova (2005) talks about how the magic circle of a game can be perforated, which, as a result, enables the online and offline realities to mix. In UE, the design was made to open up for players' social fantasies by including them in the make-believe of the narrative. At the same time, the design was also intended to provide players with the experience of being a social innovator. This was achieved by letting them 'play' with real world problems, asking them to use their real name, telling them that this is 'for real', and asking them to not only learn and imagine but also act throughout the game and produce a detailed plan for post-game action. In that sense, the game prepared players for a future as a social innovator while also offering a safe space to practice.

Another way that UE was designed for empowerment is that the game offered a goal-oriented structure or process where actionable next steps paved the way to success, and tokens were on offer (badges, power points, and awards) for completed missions. Players were also offered a great deal of 
freedom to shape their own game, as the game had very few explicit rules and the severity of the problems dealt with ensured a high level of uncertainty. This means that there was no one right answer to the problems, just an infinite number of possible solutions. The narrative, along with the missions and quests, was designed to ensure that the players knew what to do next. As the game developed and the missions and quests became tougher, the players could also experience themselves becoming increasingly proficient. In other words, the players began to grow and develop as social innovators.

A third and very central way in which UE's design supported player empowerment was the manner in which the game facilitated the forming of a social network among its players. This was done through the narrative of the game, which told players that social innovation requires teamwork; through the complexity of the missions, which led players to collaborate; and by facilitating player communication via the discussion forum and players' personal pages. As the game developed and players started to befriend each other, a network emerged that was transferrable to the physical world and enabled them to share ideas, knowledge, and other resources.

\section{Methodology and methods}

The data presented in this chapter stem from an in-depth single case study of UE. The study is based on a mixed-method approach, and the methods used are:

- non-participatory observations on the UE site after the game ended;

- a document analysis of an evaluation report on UE, written by the Natoma Group for the World Bank;

- open and semi-structured qualitative interviews conducted via email and Skype with twelve winners of UE as well as one face-to-face interview with Robert Hawkins, senior advisor at the World Bank and executive producer of the game;

- a social network analysis of the players' in-game social network, which was conducted using the NodeXL software.

The aim of the case study was to produce a rich, context-dependent description of a game that provides a nuanced insight into how the game design enabled its players to become post-game social innovators. The ontological starting point for this research is that it is impossible to capture one 'true' social reality because the social world only shows itself through representations or interpretations and because no one experiences or interprets things 
in the same way. It is therefore necessary to operate with a pluralistic concept of reality (Denzin, 2012; Greene, 2012; Pearce, 2012). The epistemological consequence of this pluralism is that the knowledge created about the social world should be seen as an interpretation, i.e., a construction, or one story among several possible stories. In such a constructivist, ontological, and epistemological framework, the aim of the researcher is not to produce 'truth' but to bring 'breadth, complexity, richness, and depth to any inquiry' (Denzin, 2012). Such an imperative calls for a methodology that makes room for multiple voices, differences, and dialogue. The methods chosen for the case study are qualitative and quantitative. Together, they represent different ways of looking at the same phenomenon and different voices (Denzin, 2012).

In the analysis, the participants are given pseudonyms when they are quoted. This is not so much to hide their real identity but to underline that the analysis should be seen as the author's interpretation and not necessarily as the opinions of the respondents.

\section{Theory}

The analysis is based on the third generation of activity theory (3GAT) (Engeström, 1999; Engeström, 2001, 2008; Engeström, Engeström \& Kärkkäinen, 1995; Engeström, Miettinen \& Punamäki, 1999; Engeström \& Sannino, 2010). AT is a social-learning theory based on the idea that the interplay between humans and technologies or mediating artifacts creates a dialectic relationship that drives cognitive, emotional, and material development.

AT should be seen as an evolving 'philosophical and cross-disciplinary framework for studying different forms of human practices as development processes, both individual and social levels interlinked at the same time' (Kuutti, 1996).

The first generation of AT (1GAT) was produced by Vygotsky (1896-1934). His idea was that activities are the key to understanding human development and that activities involve the use of mediating artifacts. The second generation of AT (2GAT) builds on the work of Vygotsky's student, Leont'ev. Leont'ev added a social aspect — community — to the theory, as he thought that human activities and learning always takes place in communities where there is a division of labor among the participants and the interaction is guided by a set of social rules. Engeström's 3 GAT provides the theory with a network perspective-connecting different activity systems to each other. The connectedness enables a discussion of how learning and development in one system can be transferred to another activity system. 


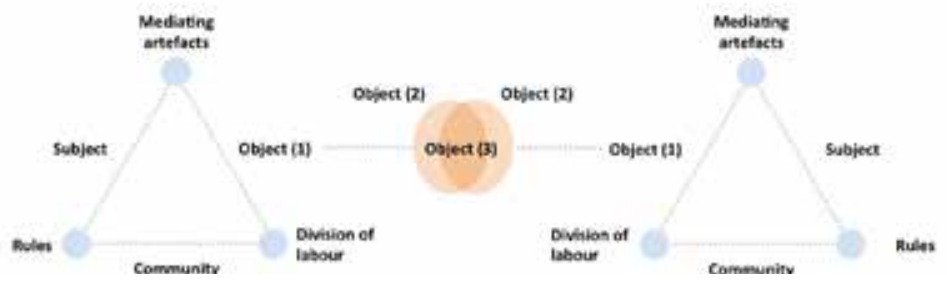

Figure 11.1: Reproduction of a 3GAT model from Engeström (2001:136).

Engeström's 3GAT can be visualized in the following way:

Engeström's 3GAT is a learning theory, but it could also be seen as an innovation theory because the focus is on the meeting of at least two activity systems where a new third and shared object is created through negotiations between the systems. This negotiation process of a new third object could be seen as a process of innovation-or at least as a process that has the potential for innovation. The new third object is placed outside and in between what could be called 'the original activity systems' and is shaped by the previous learning and goals of the activity systems involved. The third object stands in a dialectical relationship with the 'original' activity systems because it reflects back on the systems involved and influences them. With Engeström, activity systems are embedded in larger structures where new objects are created and mutual influence takes place.

Engeström's 3GAT model is interesting in relation to UE and this chapter because it enables a discussion of how the game permitted a transfer of resources from the game as an activity system to the players' post-game activity systems in the physical world. UE's goal was to empower the winners in a way that enabled them to implement their ideas in the physical world after the game ended. From a 3 GAT perspective, this means that the winners should be able to identify another activity system and be willing to negotiate a new third and shared object based on their Evokations.

\section{Analysis}

The following analysis focuses on the moment of transit and the post-game situation, where the winners have to transfer the resources from the game to the physical world and start implementing their ideas. The analysis will focus on the winners whose transfer process succeeded, but before doing so, the context of the transfer situation will be sketched using $3 \mathrm{GAT}$. 


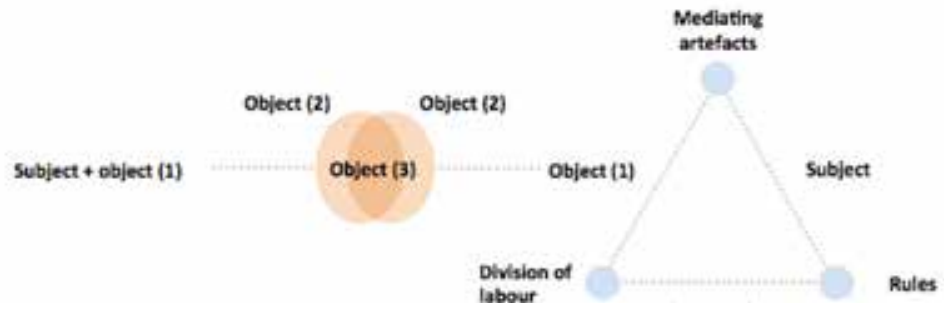

Figure 11.2: Visualization of an activity theory understanding of the post-game situation of the winners of Urgent Evoke.

\section{Game over}

When UE ended, all the players, including the winners, had to leave the game. This meant that the winners were supposed to start implementing their Evokations without having access to a platform that could host their community and facilitate their collective activities. The winners were therefore left in a vulnerable situation without the structure, resources, and community of the game at the precise moment when they were expected to act as social innovators in the physical world. An activity theory-inspired drawing of the situation could look like this:

Engeström's original model depicts a meeting between two activity systems. However, in the case of UE, the winners were left to their own devices when it came to trying to negotiate a new third object with an entire activity system. This could be understood as a moment of disempowerment, and the inequality makes it understandable that some of the winners did not manage to transfer their Evokation to the physical world.

The following analysis builds on the interviews conducted with the winners of UE. Thirty-two players won the game. Of them, eighteen have been traced and interviewed. Ten of the eighteen succeeded in turning their ideas into reality to varying extents. Of the ten successes, three that illustrate how the game design supported the players' transfers have been chosen for this chapter: the stories of Axel, William, and Jonas.

\section{Transferring self-confidence}

Axel describes himself as an introverted white man from Zimbabwe who has always had the feeling that he should change the world for the better but didn't dare say it out loud. In UE, Axel met like-minded people and it suddenly felt okay to state his desire to become a social innovator. At the end of the game, Axel decided to turn in an Evokation. As he is a programmer, his 

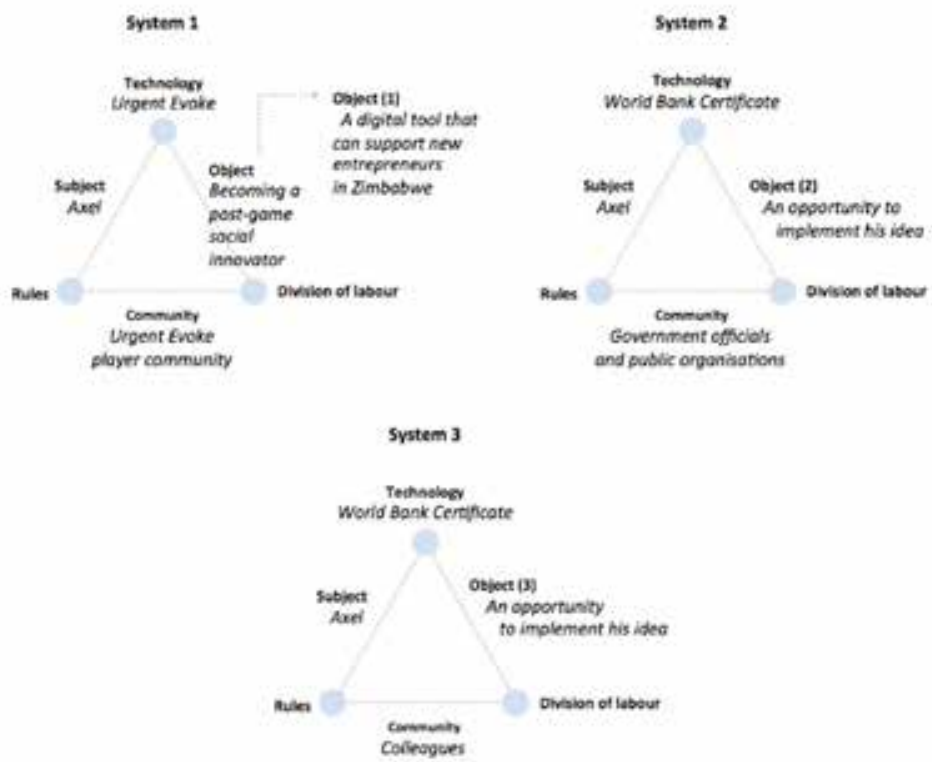

Figure 11.3: Visualization of Axel's in-game and post-game work.

idea was to produce an IT platform that could help people build their own business by creating an eco-system online where they can learn, collaborate, and build a new social network.

Post-game, Axel visited an ICT conference run by a ministry in Zimbabwe. Here, he told the other participants that he was an accredited World Bank social innovator. Mentioning this accreditation gave him access to the minister and a chance to present his Evokation. Today, Axel works for the government implementing his Evokation in a renegotiated form, where the aim is to create a digital platform where prisoners can get the support needed for them to develop business ideas and a way out of a life of crime. There is no doubt in Axel's mind that he would not be doing what he is today if it were not for UE:

[...] personally I found it [the game] builds up your confidence thatyou do have a voice and you can actually make a change. I don't think I would have had the confidence to approach the prisons and do the work projects that I'm doing now if I hadn't been through Evoke. (Axel)

Visualizing Axel's in-game and post-game work produces the following:

From a 3 GAT perspective, Axel made a successful transfer from the game to the physical world because he was capable of identifying another activity system (the prisons) that had an interest in his idea and because 
he managed to become a member of the prison activity system. He brought his object with him, which he modified to fit this system. UE empowered him by providing him with a safe space where he could practice his voice and develop the self-confidence needed to say out loud that he was capable of creating social change. Furthermore, Axel brought with him the World Bank certificate stating that he is a social innovator. Even though it had been earned in a game, this had a recognizable value outside the game and was a key to a new activity system.

\section{Transferring the object to new activity systems}

William is African and his Evokation is about starting a demo-farm where local farmers can try out new technologies and build up new knowledge that can develop and strengthen local agriculture. As well as playing UE, William is also busy with his education and knows that he can't work full time on his Evokation. He therefore had to pass it on to another activity system. However, as the game has ended, he can't find a system that has the resources or the structure needed to implement his idea. William's first challenge was therefore to raise money through the Global Giving competition:

You should have a good network to be able to get funding, to get people to donate to your cause, especially in the initiate stage, [...] I didn't have [this] at the time, so I tried to use Twitter and Facebook [...] asking people to donate to the cause [...] I think overall I got about [...] seven hundred something dollars. But then Istill had to get the extra one thousand from the World Bank [...] so I was good. Many people, well not many people but some people, managed to get to the next round [...] That's something I didn't manage to do because of my network at the time [....] (William)

William struggled to raise money for his project, just like other players, and he succeeded not because he had a large social network that he could draw on but because he used alternative technologies like Facebook and Twitter that allowed him to reach people outside his personal network.

I looked at it as, you know, in a way it was about fundraising and also trying to sellyour idea to people and see how people would respond to that. I had many random donors, which was really interesting to see; people just donating to a cause and I didn't even know them. I had some friends donating, and it was also really interesting to see that friends could really believe in my cause. It's also like a very rare thing to get people here to donate to an online cause [...] and also a lesson that I learned from that is I've been able to do many drives, like fundraising drives locally, but all my experience has been drawn from what I learned from the game. (William) 
For William, UE was more a learning experience than an entertainment technology, and the learning that began in the game can be transferred to the physical world. In William's view, there is no boundary between the in-game and out-game situation. Instead, there is a common thread running from William's experiences in the game to what he learned afterwards, because these experiences continued to form the basis for more learning post-game. He seemed surprised about what he was able to achieve, and his positive experience of winning the game seemed to be reinforced by him getting both strangers and friends to donate online to his project.

After the Global Giving contest, William knew that he didn't have enough money to start the demo-farm described in his Evokation. Instead, he approached a women's farming group in his local environment. He wanted to see what they would do if they got the thousand dollars he had raised. Together, they came up with an idea of micro-loans for local female farmers. The women weren't as organized as William would have liked them to be, but together they developed a structure to assess whether a loan applicant was eligible or not.

They had a group, which was, I would say, not very active in terms of coming together, planning together, cooperating, and what I wanted to achieve with my project was to see a women's group that really works together [....] From my childhood I learned that money is something that brings people together, but it can also separate them. So [...] being able to give people these small loans would bring them together, because every time someone wanted the money, they would come to the group and say, 'Look, I want to grow beans', and they would say, 'How much beans do you want to grow?', and they would say, 'Probably if you give me enough to buy ten kilos, I would be happy', and they would say, 'Doyou have the land?', and they say, 'Yes', and then they would say, 'Okay, first we come and inspect your land then we would...'. So eventually, it would turn out that they get more info and then in a way they keep following up from time to time and for me that was something that I really wanted to see and it's exactly how they are taking it on. (William)

Looking at William's story with 3 GAT, it's clear that he's aware that he needs an activity system to implement his Evokation successfully. He has chosen to collaborate with a network he already knows. He was also willing to let go of his original idea and instead negotiate with the women about how the funding he raised could best be put to use. Through the negotiations, William and the women managed to create a new shared third object - providing micro-loans to female farmers. What's more, during the negotiations, the women's network began to resemble an activity system with an object, rules, and a division of labor. 

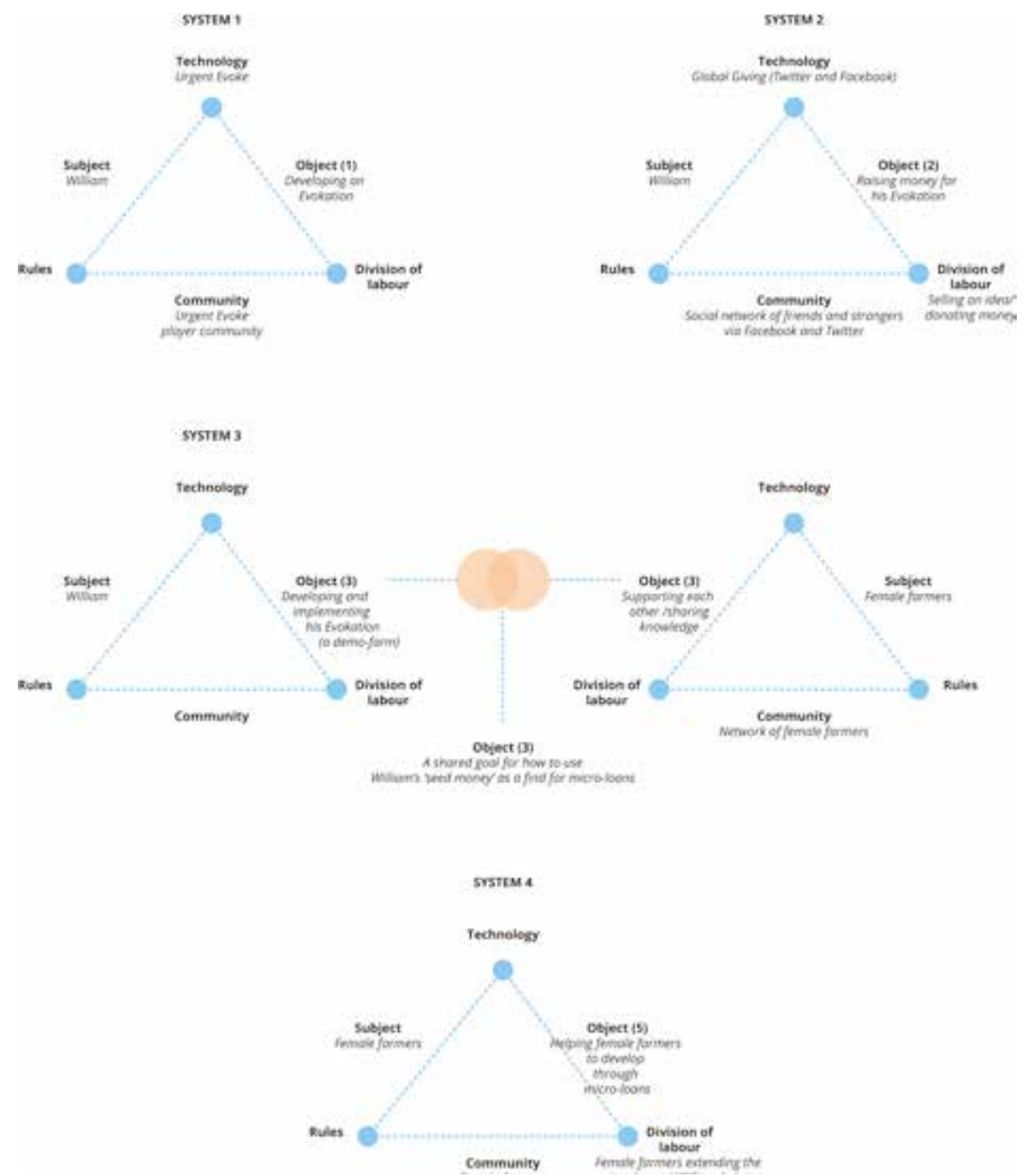

Figure 11.4: Visualization of William's in-game and post-game work.

The following sketch shows William's in-game and post-game situations. Again, no negotiation took place between the two activity systems postgame, but William managed to construct a new activity system around a new third object.

As well as helping him become aware of his own capacity as a social innovator and fundraiser, William gained from the game an understanding of the structures and level of collaboration needed for a social network like that of the women farmers to be able to function as the learning community that he would have liked his demo-farm to be. 


\section{Continuing the development process}

Jonas's story starts with him and some other players deciding to work together on the implementation of their ideas from the game. This could be seen as an attempt to create a new activity system based on the old player community, but the collaboration did not last that long:

I guess because we [...] didn't see the immediate feedback that we got in the game, like 'you did this - great!' and you know, like points and people encouraging you. It was like everyone lost momentum and then we [...] lost coordination and plus this, there wasn't a very clear vision of what needed to be done. (Jonas)

Jonas describes how the group lost momentum because the instant feedback from other players and the clear goal structure of the game were no longer there. Yet losing momentum with the group of former players didn't stop Jonas. He kept working on his own Evokation, which was focused on urban gardening. He experimented on his own rooftop. After some time practicing, he began helping friends to build their own rooftop gardens. He used his offline social network to build a community around his idea and, through this network, disseminated his vision and know-how.

After some time, Jonas got a new job and had to move to another part of the country. Moving meant a change of scenery, and Jonas found himself in a situation where he had to relearn his newly established urban farming skills and build a new social network:

$[. .$.$] the weather's very different from here, so everything changes; I had to$ re-learn a lot of the things I'd learned. But it was good in another sense because I met people there and I learned how to grow this there in that weather and stuff like that (Jonas).

Jonas learned how to farm in new weather conditions, and he also managed to build a new community. He started to dream bigger dreams based on these positive experiences:

I have another project right now, [...] and I think it's learning a lot from these experiences and it's expanding a lot; it's not going to be only about urban farming; it's going to be about the May Cube Movements, you know, like in a broad sense like farmers, and people doing urban farming, and people doing ${ }_{3} D$ printing, and people doing crafts, you know, trying to learn from this lesson and try to keep going. (Jonas)

Since UE, Jonas has gone through several learning experiences, a process he described in the following way: 

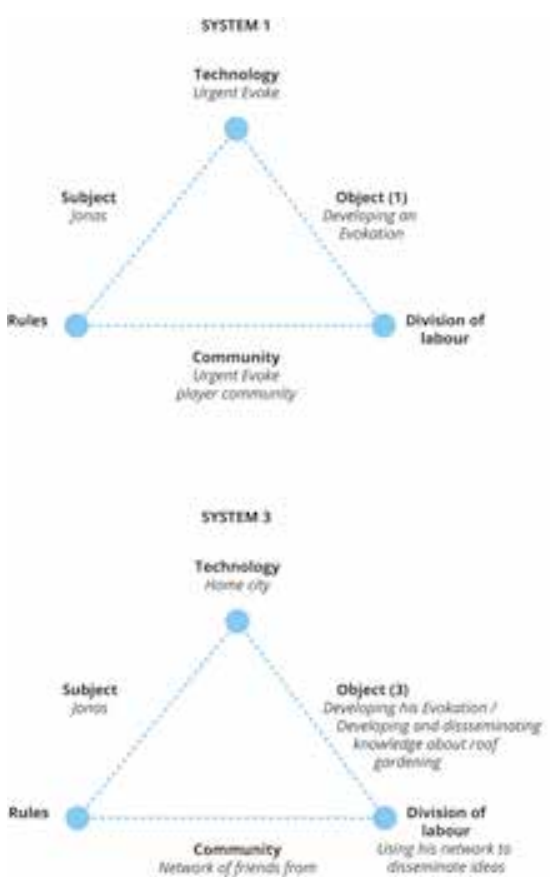
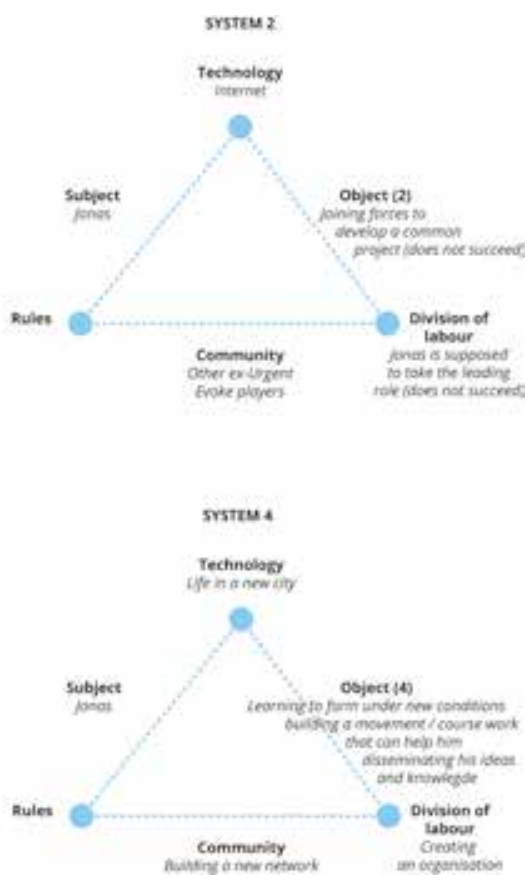

Figure 11.5: Visualization of Jonas's in-game and post-game work.

I see it as three steps; first, I learned a lot in Urgent Evoke; second, I learned a lot with my own project thanks to Urgent Evoke and then, hopefully, I will take all those lessons into this project. (Jonas)

As with many of his game colleagues, Jonas's way of handling the implementation of his Evokation also calls for changes in Engeström's model:

From a 3 GAT perspective, Jonas's process indicates that he transferred elements that he met through the UE game to his own post-game process. He was aware that he needed to build up both the core skills required to be an urban gardener and a social network to help him disseminate his knowledge and ideas. UE provided him with a way to handle his own developing process as a social innovator, which was an approach that allowed him to expand his confidence, skills, and ideas step by step:

So it [the game] has taken me from how I saw myself there [...] to someone that can have a larger impact. [...] I think that's so nice, [...] I haven't said this out loud, but I'm thinking about it, and it's such a nice change [...] in my own perception of myself. So now I do believe I'm a social innovator [...] Maybe I knew before that I had an impact on a smaller scale [...] but not in such a large scale and with subjects that aren't necessarily related to what I went to school for. (Jonas) 


\section{Conclusion}

At the start of this chapter, Figure 8.1 depicts Engeström's third generation activity theory (3GAT). As noted then, the model provided a framework for understanding how players could move from an in-game to a post-game situation and in so doing conduct a successful transfer of knowledge and resources from the game to the physical world.

Yet, as the analysis has shown, the post-game reality of the players did not mirror the theoretical model because the empowering structure of the game vanished when it came to an end. This meant that the players lost the activity system that had supported their development as social innovators at the time when they should have been starting to perform this role offline. Designing the game to end at this crucial point in the players' development process created a situation of disempowerment and made it even more difficult for the winners to conduct the intended transfer of resources and ideas from the game to the physical world. Despite this, however, the analysis has also shown that some of the winners still managed to implement their Evokation post-game, e.g., Jonas's urban gardening project, William's network of female farmers, and Axel's digital platform for prisoners with business ideas.

What is common to the winners who succeeded post-game is that even though UE as an activity system ceased to exist, the players felt that they had received attention and social recognition from the player community during the game, which gave them the courage and desire to see themselves as post-game social innovators, to believe in their own voice and ideas, and to continue developing their ideas and themselves post-game.

The winners interviewed not only gained self-confidence and a learning capacity from the game but also ideas, a social network, a World Bank certificate stating that they are certified social innovators, seed money and money from the Global Giving crowd-funding competition, and maybe also a personal mentor. All these are social and material resources that supported their post-game endeavours.

Together, these mental, social, and material resources that were transferred from the game to the physical world played an important role in the empowerment of the winners. Yet the analysis highlights one other very important thing that the players seem to have taken with them from the game, namely an understanding of how social innovation needs to be embedded in an activity system in order to take off. Looking at the winners' interviews and their post-game strategies, it's possible to recognize in all of them a systemic understanding of human learning and development. This 
can be seen in Jonas's way of building his project in stages, starting with friends in his home town and ending with a network of total strangers in a new city, and in William and Axel's ways of identifying existing networks that could adopt their idea or within which they could develop their idea further.

In conclusion, it's clear that UE teaches us that, in order for a game design to facilitate the transfer of resources from the online to the offline world and thereby empower players to become post-game social innovators, it's not enough to produce a design that provides players with access to a mix of mental, social, and material resources; the game must also be designed to be an exemplary learning environment where the design itself works as a form of meta-communication that shows the players what social structures and systemic elements are required to enable citizen-driven social innovation. In addition, UE highlighted the need to recognize the game as an activity system equal to an activity system in the physical world. This means that games should not be designed to end at the precise moment the players are being asked to transfer their ideas to the physical world. Instead, we should think of games as infinite platforms to which players can return and reconnect with the resources made available to them throughout the game. Indeed, by keeping UE alive, the design would have provided its winners with support in the difficult transfer process.

\section{References}

Castronova, E. (2005). Synthetic Worlds: The Business and Culture of Online Games. Chicago, IL: University of Chicago Press.

Clark, V.L.P., \& Creswell, J.W. (2007). Designing and Conducting Mixed Methods Research. Thousand Oaks, CA: Sage Publications.

Connolly, T.M., Boyle, E.A., MacArthur, E., Hainey, T., \& Boyle, J.M. (2012). A Systematic Literature Review of Empirical Evidence on Computer Games and Serious Games. Computers \& Education, 59(2), 661-686. doi:10.1016/j.compedu.2012.03.004.

Costikyan, G. (2002). I Have No Words \& I Must Design: Toward a Critical Vocabulary for Games. In F. Mäyrä (ed.), Computer Games and Digital Cultures Conference Proceedings, pp. 9-35. Tampere, Finland: Tampere University Press.

Denzin, N.K. (2012). Triangulation 2.o. Journal of Mixed Methods Research, 6(2), 8o-88. https://doi.org/10.1177/1558689812437186.

Dervin, B. (1998). Sense-Making Theory and Practice: An Overview of User Interests in Knowledge Seeking and Use. Journal of Knowledge Management, 2(2), 36-46. https://doi.org/10.1108/13673279810249369. 
Donovan, T., \& Garriott, R. (2010). Replay: The History of Video Games. Lewes, England: Yellow Ant.

Engeström, Y. (1987). Learning by Expanding: An Activity-theoretical Approach to Developmental Research. Helsinki: Orienta-Konsultit Oy.

- (1999). Communication, Discourse and Activity. Communication Review, 3(1-2), 165-185. https://doi.org/10.1080/10714429909368577

- (2001). Expansive Learning at Work: Toward an Activity Theoretical Reconceptualization. Journal of Education and Work, 14(1), 133-156. doi: $10.1080 / 13639080020028747$.

. (2008). From Teams to Knots: Activity-Theoretical Studies of Collaboration and Learning at Work. New York, NY: Cambridge University Press.

—, Engeström, R., \& Kärkkäinen, M. (1995). Polycontextuality and Boundary Crossing in Expert Cognition: Learning and Problem Solving in Complex Work Activities. Learning and Instruction, 5(4), 319-336. https:/doi. org/10.1016/o959-4752(95)ooo21-6.

— Miettinen, R., \& Punamäki, R.L. (eds.) (1999). Perspectives on Activity Theory. Cambridge: Cambridge University Press.

— \& Sannino, A. (2010). Studies of Expansive Learning: Foundations, Findings and Future Challenges. Educational Research Review, 5(1), 1-24. https://doi. org/10.1016/j.edurev.2009.12.002.

Gaible, E. \& Dabla, A. (2010). EVOKE Project Evaluation. Retrieved 11 June 2018 from www.siteresources.worldbank.org/EDUCATION/Resources/ProjectEvokeevaluation-final-16oct11.pdf.

Greene, J.C. (2012). Engaging Critical Issues in Social Inquiry by Mixing Methods. American Behavioral Scientist, 56(6), 755-773. https://doi. org/10.1177/0002764211433794.

Huizinga, J. (1955). Homo Ludens: A Study of the Play-element in Culture (2014 ed.). Eastford, CT: Martino Fine Books.

Kuutti, K. (1996). Activity theory as a potential framework for human-computer interaction research. In B.A. Nardi (ed.). Context and consciousness: Activity theory and human-computer interaction, pp.17-44. Cambridge, MA: MIT Press.

Law, J., \& Urry, J. (2004). Enacting the Social. Economy and Society, 33(3), 390-410. https://doi.org/10.1080/0308514042000225716.

Mertens, D.M. (2012). What Comes First? The Paradigm of the Approach? Journal of Mixed Methods Research, 6(4), 255-257. https://doi.org/10.1177/ 1558689812461574 .

Yin, R.K. (2009). Case Study Research: Design and Methods. Thousand Oaks, CA: Sage Publishing. 


\section{About the author}

Mette Wichmand is an Assistant Professor in the Department of Communication and Arts at Roskilde University in Denmark. Mette's research is concerned with how to build and sustain communities online with the purpose of generating civic engagement and social change. She is currently researching how social network games can empower players to become post-game social innovators. 



\title{
12. Striving 'Against All Odds' to Reduce Prejudice toward Immigrants and Refugees
}

\author{
Chad Michael Wertley \& Jordan Soliz
}

\begin{abstract}
The recent public and political response to immigration and refugee asylum around the world reveals that prejudice toward migrants remains a preeminent societal problem. In response to the growing political unrest towards migrants, The United Nations Refugee Agency (UNHCR) developed the web-based video game, Against All Odds, which has players take on the role of a refugee and experience the struggles they go through. Thus, the purpose of the current study is to investigate the potential of intergroup contact using a video game (i.e., Against All Odds) in changing attitudes toward migrant populations - specifically, refugees. In addition, this study explores four potential affordances of the media in the contact space that may mediate the change.
\end{abstract}

Keywords: intergroup; contact space; prejudice; refugees; video games

Imagine that, while peacefully protesting government policies, you are detained by law enforcement and interrogated on your allegiance to the government. You answer affirmatively to avoid imprisonment - or worsedeath. Upon release, you decide that fleeing your homeland is the best option to assure survival. You quickly pack necessities and sneak past guards in the dark of night. Your trip through the countryside is wrought with danger, as others like you are smuggled across the border in a dilapidated truck. Escaping your homeland is just the start of your struggle, as you are now a stranger in an unfamiliar land. You must find somewhere to stay, learn the language and culture, and get a job-all in a place where people view you

Hera, T. Dela, J. Jansz, J. Raessens, B. Schouten, Persuasive Gaming in Context. Amsterdam: Amsterdam University Press, 2021 DOI 10.5117/9789463728805_CH12 


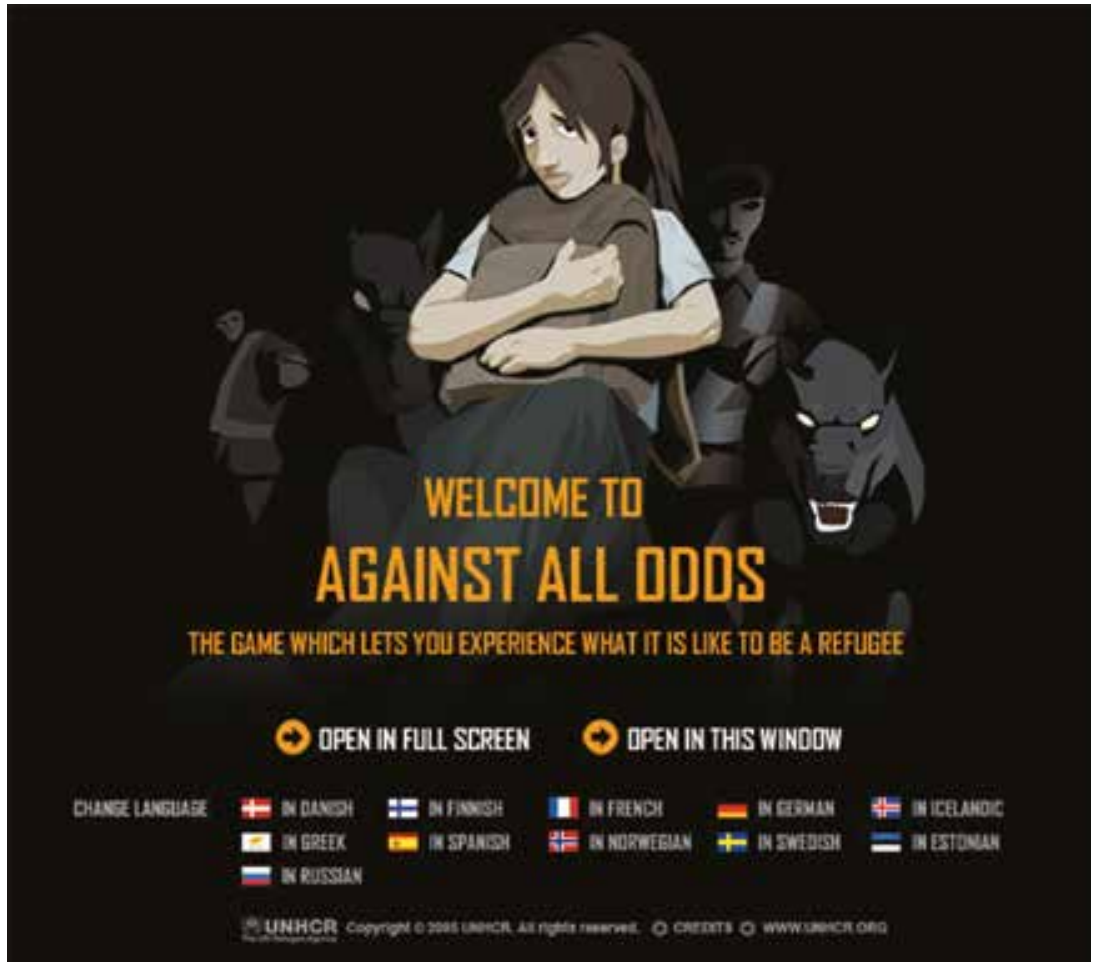

Figure 12.1

suspiciously. These are the lived experiences from 'people you see every day, but you don't really see them' at all (UNHCR, 2006). This is your journey as a refugee in the persuasive video game Against All Odds (see Figure 12.1).

The recent public and political response to immigration and refugee asylum around the world reveals that prejudice toward migrants remains a preeminent societal problem. Intolerance of immigrants and refugees is a recurrent issue, as they are often perceived as economic and cultural threats to a country's well-being (Bansak, Hainmueller \& Hangartner, 2016; Fuchs, 1990; Schweitzer, Perkoulidis, Krome, Ludlow, \& Ryan, 2005). In response to the growing political unrest toward migrants, the United Nations Refugee Agency (UNHCR) developed the web-based video game, Against All Odds, which has players take on the role of a refugee and experience the struggles described above. The aim of the game is to positively influence people's attitudes and beliefs about the refugee crisis through this simulated contact.

An extensive body of research supports the notion that positive interactions between members of disparate groups can reduce prejudicial attitudes and behaviors (Pettigrew \& Tropp, 2006). Video games have the potential to 
be a viable method to facilitate positive contact with 'the other' (Gentile et al., 2009; Greitemeyer \& Mugge, 2014), as it is a very adaptive medium when it comes to conveying social cues and enabling interactivity, both of which are vital elements in facilitating positive contact (Amichai-Hamburger \& McKenna, 2006). While the literature on the effects of gameplay on attitude change toward negatively 'othered' groups is growing, the extant research has inconsistent results and too often does not examine the drivers of the change. The purpose of the current study is therefore to investigate the potential of intergroup contact using a video game (i.e., Against All Odds) when it comes to changing attitudes toward migrant populations-specifically, refugees. In addition, this study explores four potential affordances of the media in the contact space that may mediate the change.

\section{Theoretical background}

\section{Intergroup theory}

As the root of this conflict resides in the social construction of an 'us' and 'them' (i.e., nationalists/migrants), viewing the conflict through an intergroup perspective provides unique insight into both the formation and resolution of group bias. Intergroup theory expounds the processes and functions of categorizing the self and others into social ingroups and outgroups. While categorization is not inherently problematic, one concerning effect is outgroup discrimination. This appears to occur intrinsically, as the mere perception of belonging to a group activates group bias (Tajfel, 1970).

As part of their social identity theory, Tajfel and Turner (1979) propose that ingroup favoritism and outgroup discrimination are rooted in selfconcept. People strive to achieve a positive social identity, so they favorably compare themselves to a relevant outgroup. If, however, one's social identity is threatened by such an outgroup, then the ingroup members are motivated to behave in ways that enhance their positive distinctiveness over the outgroup. Some common reasons for perceiving another group as threatening include competition over scarce resources, changes to cultural values and norms, and negative stereotypes of the other (Stephan \& Stephan, 2000). Despite the relative ease of perceiving other groups (especially migrants) as threatening, prejudice should not be viewed as an inevitable conclusion.

Allport (1954) contended in his highly influential 'contact hypothesis' that negative bias can be reduced, provided that contact occurs under the right conditions. Positive contact can be achieved when members of both 
groups interact in a situation where they have equal status, cooperate to achieve a common goal, and are supported by a third, authoritative party. The contact hypothesis has since been developed into theoretical propositions (Brown \& Hewstone, 2005; Hodson \& Hewstone, 2013; Pettigrew, 1998), and the main argument that positive intergroup contact can reduce prejudice has received strong empirical support (Pettigrew \& Tropp, 2006).

\section{Intergroup contact in video games}

Video games appear to be well positioned to provide a space that is conducive to positive intergroup contact. To successfully play a game, the player must accomplish the goals set forth by the game's narrative. For the player to win, the avatar (i.e., 'the other') must also win. The game objectives for the avatar are also the player's objectives. This should facilitate the conditions of pursuing a common goal and cooperation, while the narrative structure and gameplay mechanics provide institutional support for continued gameplay.

The following notable studies lay the groundwork on the potential of persuasive games to affect attitudes and behaviors toward 'the other'. Peng, Lee, and Heeter (2010) explored the effect that playing the persuasive video game Darfur is Dying had on participants' willingness to help. Those who played the video game expressed a greater willingness to help than those who either read an informationally comparable text or watched a video of the gameplay. Using the game Peacemaker, in which a person makes decisions to maintain peace between Israel and Palestine, Alhabash and Wise (2012) found that both implicit and explicit bias were reduced when participants played as the group opposite to their a priori attitudes, but the game had no effect on bias if the participants played as their favored group. Gutierrez et al. (2014) also measured implicit bias, finding that playing the game Fair Play reduced racial bias but only for those players who rated high on empathy toward the game character. Additional research has also shown mixed results on the effectiveness of games when it comes to influencing attitudes toward the poor (Ruggiero, 2015, analyzing Poverty is Not a Game), civic engagement on poverty issues (Neys, Van Looy, De Grove \& Jansz, 2012, analyzing Spent), and reducing racial prejudice (Behm-Morawitz, Pennell \& Speno, 2016, analyzing The Sims Social).

These studies demonstrate the potential of video gameplay to positively affect prosocial attitudes and behaviors; however, the extant research lacks a systematic approach to identifying the process through which change occurs. Understanding the factors that drive attitude and behavior 
change in a game environment may help to explain the mixed results above as well as provide insight into how to develop more effective persuasive games.

\section{The contact space framework}

In the 'contact space framework' (CSF), Harwood (2010) contends that differences in contact results may be due to differences in the mediation potential of the contact environment (i.e., the contact space). The CSF provides two dimensions consisting of both a structural and psychological characteristic. Each of the four CSF components and the potential effects they have on prejudice change are summarized below.

Self-involvement: Self-involvement concerns the level of active participation one has during the contact situation. Active participation induces greater and more persistent attitude and behavior change than passive observation or reading (Janis \& King, 1954; Watts, 1967). Unlike in static media such as books and movies, video games allow for active participation, as the player is influencing what is happening in the game world (Klimmt, 2009).

H1: The greater an individual's self-involvement, the greater the reduction in prejudice.

H2: Individuals perceive themselves as more actively involved when playing a video game than when they watch a video or read a text.

Identification: Identification occurs when an individual imagines him/herself as another (Cohen, 2001). Through this process, individuals experience the events as if they actually happened to them, resulting in empathy and perspective-taking. Identification may more readily occur in video games, since they require the player to perform the roles of the other, merging the self with the avatar (Klimmt, Hefner \& Vorderer, 2009). Performing the roles of others may lead to a greater sense of identification and thus greater attitude change.

$\mathrm{H}_{3}$ : The greater an individual's level of identification, the greater the reduction in prejudice.

$\mathrm{H}_{4}$ : Individuals identify more with a mediated 'other' when playing a video game than when watching a video or reading a text. 
Richness: Richness is conceptualized as the total number of communication cues available during the interaction and the potential of those cues to afford immediate feedback (Daft \& Lengel, 1984). As the richness of the communicative environment increases, the ambiguity of the message is reduced, increasing shared meaning and understanding. Given this assumption, the richer the environment, the greater the potential reduction in prejudice. Video games can be very rich environments, offering realistic representations of the physical world, stimulating all the senses, and providing immediate feedback on a player's actions (Klimmt, 2009), potentially making such games a richer setting than video or text.

$\mathrm{H}_{5}$ : The greater the perceived richness of the experience, the greater the reduction in prejudice.

H6: Individuals perceive that playing a video game provides a richer experience than watching a video or reading a text.

Presence: The phenomenon of presence occurs when an individual feels that a mediated other is psychologically and physically close (Lombard \& Ditton, 1997). These feelings of closeness increase one's emotional responses (Lombard \& Ditton, 1997), and have been found to be positively correlated with players' emotional reactions toward characters in video games (Ravaja et al., 2004). Presence is directly related to richness, as the extent of the communication cues influence the feeling of closeness toward another. Given this relationship, video games should induce a greater sense of presence than video or text.

$\mathrm{H}_{7}$ : The greater an individual's sense of presence, the greater the reduction in prejudice.

H8: Individuals perceive a greater sense of presence when playing a video game than they do when watching a video or reading a text.

The preceding hypotheses presume that the richness of the contact environment, self-involvement during the contact, and perceptions of identification and presence with the other are linked to prejudice change, meaning that the greater each of the components, then the greater the prejudice change. Furthermore, these hypotheses argue that these dimensions will be greater in video games compared to video and text. Therefore, we suggest the following:

H9: Prejudice reduction is greater for individuals who play a video game than for those who either watch a video or read a text. 


\section{Method}

\section{Participants}

Participants were recruited at a large Midwestern university in the United States through a call for volunteers. Respondents signed up for a lab time and were compensated with a course credit in conjunction with their instructor. The sample consisted of 197 students (99 male, 98 female) ranging in age from 19 to $33(M=20.9, S D=2.11)$. The majority of the participants reported identifying their race/ethnicity as Caucasian (73.6\%), followed by Asian (14.2\%), African-American (4.6\%), Hispanic (4.1\%), and Other (3.0\%).

\section{Procedure}

To examine the research questions and hypotheses, a one-factor betweensubjects experimental design was developed consisting of three treatment conditions (i.e., text, video, and video game), along with a control group. To reduce external distractions, the study was performed in a lab. The participants completed the entire study using an online survey program. The first part of the study consisted of a pre-test questionnaire consisting of three prejudice measures and demographic questions. Next, the participants were randomly assigned a condition by the survey software. They were then asked to spend 15 minutes completing the assigned task. Time was controlled by disabling the ability to continue with the study after the 15 minutes had expired. Upon completion of the task, the participants answered the post-test questionnaire, which consisted of the same prejudice measures as well as measures for each contact space component.

\section{Conditions}

All the materials for each of the three treatment conditions came from the UNHCR website (http://www.unhcr.org). Using materials created by the same source allows for a parallel structure between the conditions, as the themes addressed within each of these materials are similar. Regardless of which treatment the participants were assigned, they experienced refugee stories addressing the challenges of leaving one's home and starting a new life in a foreign country. The story format of these materials enables the audience to 'live' the refugee's experience. For the control condition, the participants were asked to play the puzzle game Sudoku as the non-related task. 


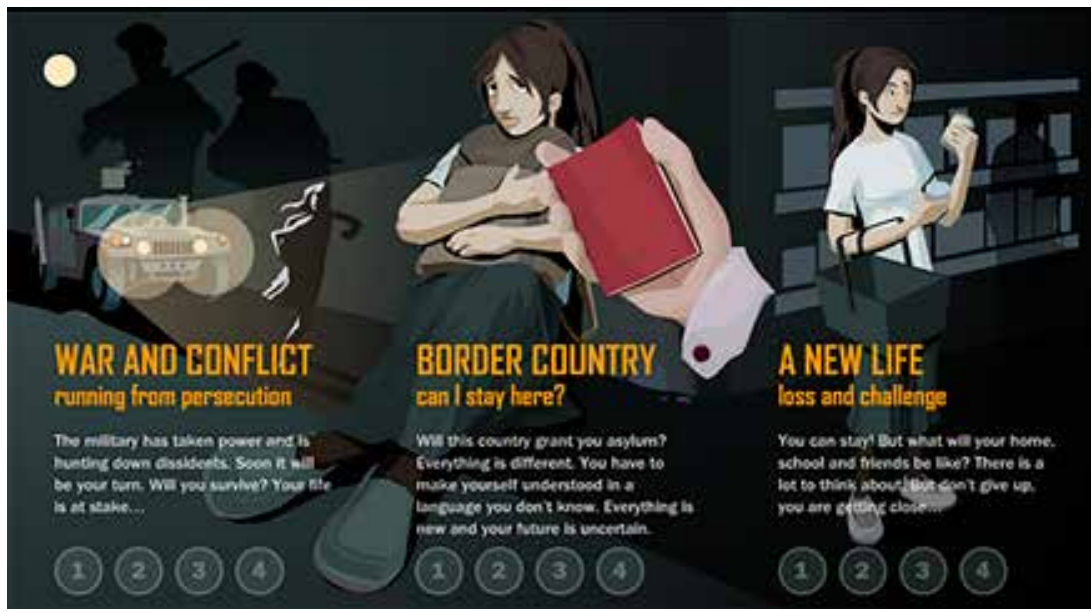

Figure 12.2

The participants assigned to the text condition read pre-selected stories from the UNHCR 'Stories' webpage (http://www.unhcr.org/en-us/stories. $\mathrm{html})$. The texts were written by UNHCR staff writers from interviews with a refugee and feature a photo of him/her. Those assigned to the video condition watched pre-selected videos from the UNHCR YouTube channel, 'USA for UNHCR' (https://www.youtube.com/c/USAforUNHCR). The video features a refugee telling their story, along with footage from his/her home country. For the video game condition, the participants were asked to play the online video game Against All Odds (www.playagainstallodds.ca). In Against All Odds, the player takes on the role of a refugee and plays through twelve episodes (see Figure 12.2) depicting persecution and flight from one's native country to seeking asylum and starting a new life in a foreign country, as detailed in the chapter's introduction. The gameplay is point-and-click, and each episode uses either a first-person or third-person perspective.

\section{Measures}

Prejudice measures: Prejudice was assessed using three measures in order to explore different facets of prejudice: general feelings, evaluative attitudes, and altruistic actions. For all measures, higher scores indicate more positive attitudes or behaviors (i.e., less prejudice). Haddock, Zanna, and Esses' 'feelings thermometer' (1993) was used to assess general feelings. The feelings thermometer is a one-item measure in which the participant moves a slider to a number ranging from o (extremely unfavorable) to 100 (extremely 
favorable) that best represents their general feelings toward immigrants and refugees.

Evaluative attitudes were measured using the 11-item 'Prejudicial Attitudes Scale' (Stephan, Ybarra, Martinez, Schwarzwald \& Tur-Kaspa, 1998). The participants rated the items on a five-point Likert scale $(1=$ strongly disagree, 5 = strongly agree) across a variety of dimensions (e.g., sympathy, dislike; $\alpha=.86$ and .90 for the pre- and post-test).

Finally, a scale was developed to assess altruistic intentions. The participants indicated their likelihood of performing various behaviors in support of refugees and immigrants by rating four items along a five-point Likert scale ( 1 = strongly disagree, 5 = strongly agree; e.g., 'I am interested in volunteering for immigrant and refugee charity organizations', 'I would support a one cent sales tax increase to support immigrant and refugee relief programs in my community'). An EFA using a principal component analysis with Varimax rotation revealed a one-factor solution: Eigenvalue $=2.616$, $65.406 \%$ of variance explained ( $\alpha=.82$ and .84 for the pre- and post-test).

Contact space measures: Self-involvement was assessed by adapting the 'involvement' factor from Kumar and Benbasat's 'para-social presence questionnaire' (2002). The involvement factor explores how the level of interactivity the user has with the medium affects positive attitudes toward the interaction. It includes eight items on a five-point Likert scale ( 1 = strongly disagree, 5 = strongly agree; e.g., 'I had physical reactions [eg. made a sound or facial expression] because of what I experienced in the media environment'; $\alpha=.87$ ).

Identification was assessed with Peng's adaptation (2008) of Cohen's 'Identification Scale' (2001). The participants rated eleven statements on a five-point Likert scale ( 1 = strongly disagree, 5 = strongly agree; e.g., 'I feel I understand the events in a way similar to how the character understood the events'; $\alpha=.86$ ).

Richness of the media experience was determined using the 'social richness' factor from Lombard et al.'s 'temple presence inventory' (2000). Users are asked to assess the effect that the medium itself had on their experience. The measure consists of seven semantic pairs on a five-point scale (e.g., 'distant - close'; $\alpha=.81$ ).

Presence was measured using the 'social presence - actor within a medium' factor from Lombard et al.'s temple presence inventory. The scale assesses the degree to which an individual feels as if the mediated 'other' is physically and personally present during the encounter. The scale consists of six items on a five-point Likert scale $(1=$ never, $5=$ very often; e.g., 'How often did it feel as if the characters were talking directly to you?' $\alpha=.81$ ). 


\section{Results}

\section{Prejudice change by condition}

Prior to our analysis, initial equivalence was assessed to confirm that attitudes and beliefs toward immigrants and refugees were similar among the randomly assigned groups. The results indicated that there were no significant differences between the groups prior to the manipulation conditions (Wilks' $\left.\lambda=.97, F(9,465)=.73, p=.683, \eta^{2}=.01\right)$

$\mathrm{H} 9$ predicted that prejudice toward immigrants and refugees would decrease more for individuals playing a video game than for those reading a text or watching a video. To assess whether the mediated contact improved the disposition toward refugees, a doubly multivariate analysis of variance on the three prejudice measures over time by condition was performed. The between-subjects factor was condition, and the within-subjects factor was prejudice measured over time. The results of the MANOVA revealed a significant multivariate time-by-condition interaction effect, (Wilks' $\left.\lambda=.78, F(9,460)=5.41, p<.001, \eta^{2}=.08\right)$. The effects of the presentation mode on prejudice was examined further by conducting a 4 (condition) $\mathrm{x} 2$ (time) ANOVA for each prejudice measure, with follow-up pairwise comparisons of the pre- and post-test scores using Fisher's LSD procedure $(p=.05)$. Descriptive statistics are provided in Table 1.

The pre- and post-test comparisons of each prejudice measure yielded the same results: a significant main effect for time (general feelings $F(1,191)$ $=72.59, p<.001 ;$ evaluative attitudes $F(1,193)=47.25, p<.001 ;$ altruistic intentions $F(1,193)=36.27, p<.001)$. The participants experienced a positive change (i.e., less prejudice) after the manipulation, while no significant change was reported in the control condition. Next, interaction effects were analyzed to discover if there were differences between conditions over time.

The general feelings measure had a significant time-by-condition interaction, $(F(3,191)=7.50, p<.001)$. Conducting a simple effect analysis using Fisher's LSD procedure $($ LSD $m m d=2.92)$ revealed the pattern that all three treatment groups had significantly more positive feelings toward immigrants and refugees than the control group following the manipulation task. However, both the text and video conditions had significantly higher scores than the video game condition (see Table 1). Hy is therefore not supported for the general feelings measure.

For the evaluative attitudes, the results indicated a significant time-bycondition interaction, $(F(3,193)=10.25, p<.001)$. Again, the participants in 
Table 1: Pre- and Post-Test Means (Standard Deviations) of Prejudice Scores by Condition

\begin{tabular}{llrccc}
\hline $\begin{array}{l}\text { Prejudice } \\
\text { Measure }\end{array}$ & Condition & $\mathrm{N}$ & General Feelings & $\begin{array}{l}\text { Evaluative } \\
\text { Attitudes }\end{array}$ & $\begin{array}{l}\text { Altruistic } \\
\text { Intentions }\end{array}$ \\
\hline Pre-Test & Video game & 50 & $67.26(19.73)$ & $3.72(.51)$ & $2.81(.78)$ \\
& Text & 47 & $67.32(21.62)$ & $3.76(.56)$ & $3.06(.91)$ \\
& Video & 47 & $69.62(19.04)$ & $3.79(.55)$ & $3.06(.78)$ \\
& Control & 51 & $66.06(20.62)$ & $3.62(.50)$ & $2.90(.86)$ \\
& Total & 195 & $67.53(20.16)$ & $3.72(.53)$ & $2.95(.84)$ \\
Post-Test & Video game & 50 & $72.84(19.06)$ & $3.85(.59)$ & $3.10(.82)$ \\
& Text & 47 & $76.45(19.79)$ & $4.10(.64)$ & $3.34(.89)$ \\
& Video & 47 & $79.00(14.66)$ & $4.05(.52)$ & $3.40(.83)$ \\
& Control & 51 & $66.92(20.99)$ & $3.59(.53)$ & $2.79(.89)$ \\
& Total & 195 & $73.65(19.24)$ & $3.89(.60)$ & $3.15(.88)$ \\
\hline
\end{tabular}

Note: Higher scores indicate more positive perceptions and evaluations.

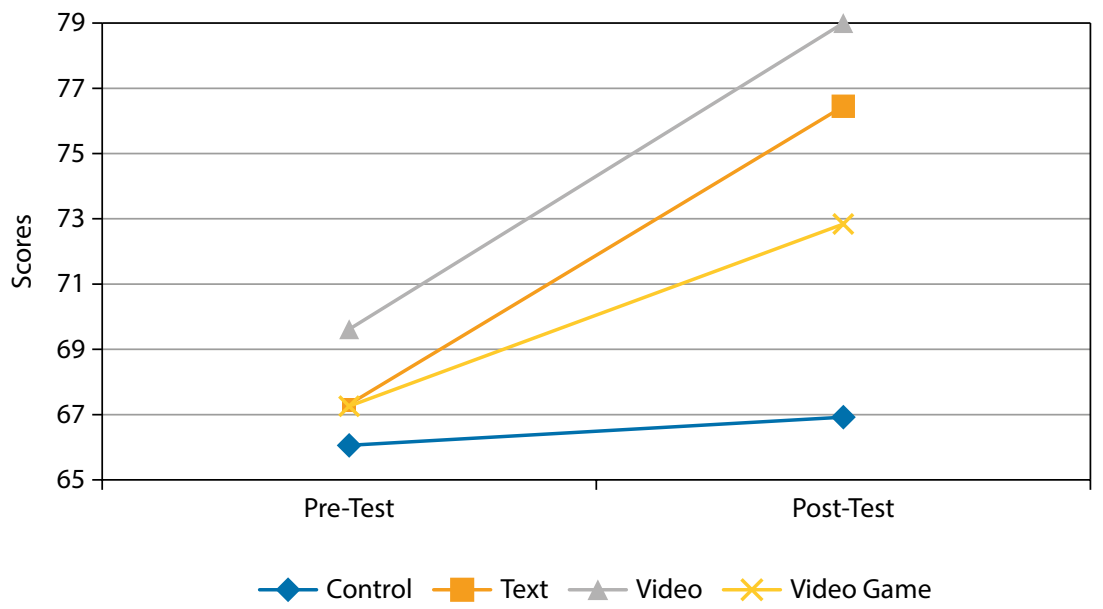

Figure 12.3: Pre- and post-test general feelings scores by condition.

all three treatment conditions experienced a significant positive change in prejudicial attitudes, with no significant change in the control condition. Exploring the post-test differences, the video-game condition had significantly lower prejudicial attitude scores than both the text and video conditions (see Figure 12.3). Hg is therefore not supported for evaluative attitudes.

Identical results occurred with the altruistic intentions measure, as there was a significant time-by-condition interaction, $(F(3,193)=9.84, p<.001)$, with the interaction pattern revealing a significant positive change in 


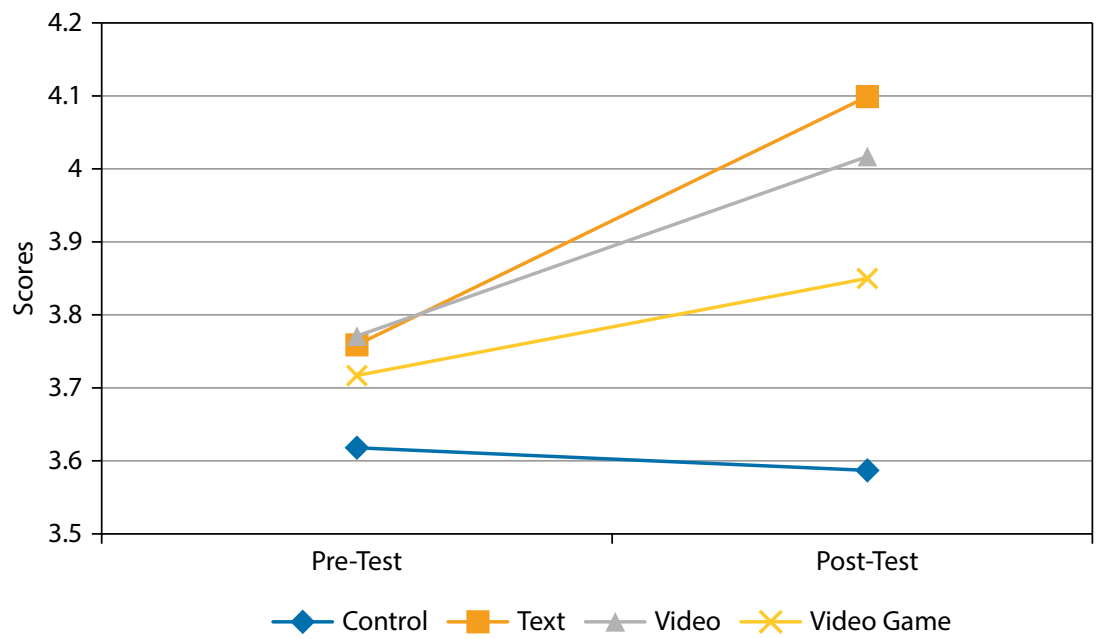

Figure 12.4: Pre- and post-test evaluative attitudes scores by condition.

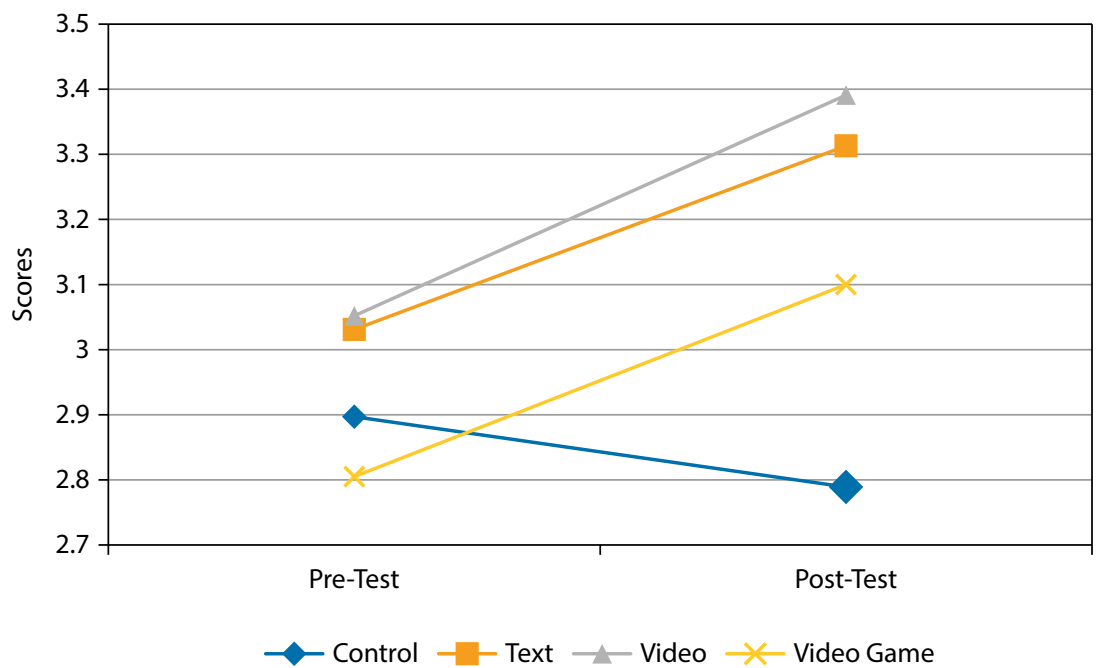

Figure 12.5: Pre- and post-test altruistic intentions scores by condition.

behavioral intentions for the participants in the treatment conditions and a non-significant change for those in the control condition (see Figure 12.4). As in the previous two results, the video-game condition had significantly lower pro-social behavior scores than both the text and video conditions. $\mathrm{Hg}$ is therefore not supported for altruistic intentions.

In summary, while all three conditions significantly reduced prejudice, the video-game condition decreased significantly less than both the text and video conditions for all three prejudice measures. As a result, Hy is not supported. 


\section{Relationship between the CSF and prejudice change}

To test $\mathrm{H}_{1}, \mathrm{H}_{3}, \mathrm{H}_{5}$, and $\mathrm{H6}$, bivariate correlation analyses were conducted between each CSF characteristic and the change in prejudice scores for each prejudice measure (see Table 2). Prejudice change was calculated by subtracting the pre-test scores from the post-test scores. $\mathrm{H}_{1}, \mathrm{H}_{3}$, and $\mathrm{H}_{5}$ were supported in that higher perceptions of self-involvement, identification, and richness were associated with positive attitude change across all three prejudice measures. $\mathrm{H}_{7}$ was not supported, as presence was not associated with attitude change.

\section{Table 2: Pearson Correlations between the Contact Space Framework Characteristics and Prejudice Change}

\begin{tabular}{lccc}
\hline $\begin{array}{l}\text { Contact Space Framework } \\
\text { Characteristic }\end{array}$ & General Feelings & Evaluative Attitudes & Altruistic Intentions \\
\hline Self-Involvement & $.34^{* *}$ & $.51^{* *}$ & $.30^{* *}$ \\
Identification & $.30^{* *}$ & $.40^{* *}$ & $.19^{*}$ \\
Richness & $.35^{* *}$ & $.38^{* *}$ & $.25^{* *}$ \\
Presence & .10 & .10 & .10 \\
\hline
\end{tabular}

Note: * Correlation is significant at the 0.05 level

** Correlation is significant at the 0.01 level

\section{Perceptions of the CSF characteristics across contact modes}

We conducted a one-way MANOVA to address $\mathrm{H}_{2}, \mathrm{H}_{4}, \mathrm{H} 6$, and $\mathrm{H} 8$, with the four CSF components as the dependent variables and the three manipulation conditions as the fixed factor. The MANOVA revealed a significant multivariate main effect for condition (Wilks' $\lambda=.72, F(8,280)=6.24, p<.001, \eta^{2}=$ .15). Descriptive statistics are provided in Table 3 , and follow-up univariate tests were conducted. For self-involvement, there were no significant mean differences in feelings of involvement among the three media conditions $\left(F(2,143)=1.23, p=.295, \eta^{2}=.02\right) . \mathrm{H} 2$ is therefore not supported. There were significant mean differences in identification $(F(2,143)=6.12, p=.003$, $\left.\eta^{2}=.08\right)$, with individuals who either read the text or watched the video identifying more with the 'other' than those who played the video game (LSD mmd $=.23$ ). $\mathrm{H}_{4}$ was therefore not supported. Similar results were found for richness $\left(F(2,143)=12.23, p<.001, \eta^{2}=.15\right)$ in that individuals who either read the text or watched the video perceived those modes as richer environments than those who played the video game (LSD mmd =.25). $\mathrm{H6}$ is 
therefore not supported. Finally, there were no significant mean differences in feelings of presence among the three media conditions $(F(2,143)=2.84$, $p=.062, \eta^{2}=.04$ ), meaning that $\mathrm{H} 8$ is not supported.

Table 3: Means and Standard Deviations of the Contact Space Framework Characteristics by Condition

\begin{tabular}{llrrr}
\hline $\begin{array}{l}\text { Contact Space Framework } \\
\text { Characteristic }\end{array}$ & Condition & N & M & \multicolumn{2}{c}{ SD } \\
\hline Self-Involvement & Video game & 50 & 3.61 & 0.74 \\
& Text & 48 & 3.79 & 0.58 \\
& Video & 48 & 3.78 & 0.58 \\
& Total & 146 & 3.72 & 0.64 \\
Identification & Video game & 50 & $3.46 \mathrm{a}$ & 0.67 \\
& Text & 48 & $3.78 \mathrm{~b}$ & 0.53 \\
& Video & 48 & $3.81 \mathrm{~b}$ & 0.46 \\
& Total & 146 & 3.68 & 0.58 \\
Richness & Video game & 50 & $3.25 \mathrm{a}$ & 0.72 \\
& Text & 48 & $3.81 \mathrm{~b}$ & 0.54 \\
& Video & 48 & $3.74 \mathrm{~b}$ & 0.57 \\
Presence & Total & 146 & 3.59 & 0.66 \\
& Video game & 50 & 2.71 & 0.81 \\
& Text & 48 & 2.69 & 0.84 \\
& Video & 48 & 2.37 & 0.75 \\
& Total & 146 & 2.59 & 0.81 \\
\hline
\end{tabular}

Note: Higher scores indicate higher perceptions of the characteristic.

Differing subscripts indicate significant difference at the 0.05 level.

\section{Discussion}

The purpose of this study was to examine the potential of a persuasive video game to reduce prejudicial attitudes toward refugees. Encouragingly, the participants who played Against All Odds experienced a reduction in prejudicial attitudes, providing credence to the persuasive capabilities of video games. However, this reduction was less pronounced than the one in those who read the text or watched the video. This is similar to the results of Van 't Riet and colleagues' study (2018) of Against All Odds, which also found text and video to be more persuasive than the game. However, they found no significant effect on video gameplay and a change in willingness to help, whereas the present study found a significant—albeit small—change in prejudicial attitudes. While the results speak to the promise of persuasive 
gaming effects, the integration of Harwood's contact space framework provides additional insight into how aspects of the medium may facilitate change.

First, self-involvement was a significant driver in the prejudice change but did not significantly differ among the three conditions. This result was counter to the hypothesis that self-involvement would be higher with the video game. This should caution us about viewing active participation as the user's ability to influence the events of the encounter. This conflates 'interactivity' with 'self-involvement', fostering the commonly held notion that video game play is more active, while reading/watching are more passive. We can expend a great deal of mental and emotional energy actively reading, watching, or playing media, or we can passively consume all three. Future research should explore which factors contribute to active participation in persuasive video games.

Second, the findings demonstrate that identification is linked to positive attitude change. The participants reported identifying with the refugees in all three conditions but did so more with refugees in the text and video than in the video game. This result was also contrary to our prediction. This is surprising, as the qualities of video games appear to be able to enhance identification; in such games, one has a monadic relationship with the game, as the player assumes the perspective of the avatar to accomplish the game's objectives (Klimmt et al., 2009). Van Looy and colleagues (2012) may provide insight into this discrepancy. Players identify with the avatar, the social group, and/or the game. Given the conditions of the experiment, it is likely that these dimensions did not have time to develop, as the players did not socialize with anyone about the game or did not have the opportunity to explore the game in more depth. Most importantly, the common factor in all three dimensions is the player's use of gaming to escape reality, and playing a game about the refugee crisis defeats this purpose.

As expected, the results indicate that the richer the participants perceived the environment to be, the greater the change in prejudice; however, the video game was viewed as less rich than the video and text. This is counterintuitive, as richness is conceived as the number of communication cues available during the contact, and the video game has more of these than the text or video. This implies that richness should be expanded beyond the number of senses activated by communication cues and that the effects of available cues should be examined. This is because too many cues can have a deleterious effect (Pavlovic \& Markovic, 2011), while a lack of cues can enhance emotions (Walther, 1996). The strategic use of available cues may therefore be more important than the number used. 
Finally, contrary to our expectations, presence was not significantly related to a change in prejudice. While we should be cautious about disregarding presence as a facilitator of persuasiveness, the findings do provide an opportunity to question its efficacy. Feeling that the other person is 'with you' may not be capturing what it is about the contact environment that facilitates attitude change. Transportation may be a more appropriate construct. Transportation focuses on the extent to which an individual is absorbed-or immersed — into another's narrative (Gerrig, 1993). The more one feels transported into a narrative, the less the narrative is discounted and the more favorably the protagonist is evaluated (Green \& Brock, 2000).

\section{Limitations}

Despite video games becoming more ubiquitous in society, the average person has vastly more everyday experience of reading text and watching video. This lack of literacy in video games may have affected the results: Those who are less competent in playing video games may have expended more effort in figuring out the game mechanics, reducing their ability to attend to its persuasive message. This could especially be true with Against All Odds, as the controls and objectives in some episodes were not always clear, making them frustrating to play.

A second limitation was the time constraint: Fifteen minutes was not enough time to complete the game but was long enough to complete the video and text. A lack of a full story could lead to a reduction in narrative coherence (Fisher, 1987), diminishing the empathy and perspective-taking effects of the game's narrative (Bochner, Ellis \& Tillman-Healy, 200o). Compounding matters, differences in gaming abilities among the participants could have led to differences in how far they progressed through the game. Variations in video-game literacy and progression through the game narrative may account for differences in the extent of prejudice change.

\section{Conclusion}

Overall, this study extends the research on the persuasive capabilities of video games in an intergroup contact scenario and illuminates specific media attributes in fostering attitude change. While the effectiveness of this particular game was limited, the study does support the notion that video games can be a viable option for facilitating attitude change. However, 
caution should be applied in attributing any effects with a specific medium. Some persuasive games will be effective, and others will not. The results reveal the need for more nuanced research into the affordances of video games as a medium, and how those affordances can best be implemented to achieve the desired goal. Certain attributes of video games may be more conducive to influencing behavior, and certain narratives may lend themselves better to being told through video games.

\section{References}

Alhabash, S.E., \& Wise, K. (2012). PeaceMaker: Changing Students' Attitudes Toward Palestinians and Israelis Through Video Game Play. International Journal of Communication, 6, 356-380. Retrieved 11 July 2018 from https://ijoc.org/index.php/ijoc/ article/view/1056.

Allport, G.W. (1954). The Nature of Prejudice. Cambridge, MA: Addison-Wesley.

Amichai-Hamburger, Y., \& McKenna, K.Y. (2006). The Contact Hypothesis Reconsidered: Interacting Via the Internet.Journal of Computer-Mediated Communication, 11, 825-843. https://doi.org/10.1111/j.1083-6101.2006.00037.x.

Bansak, K., Hainmueller, J., \& Hangartner, D. (2016). How Economic, Humanitarian, and Religious Concerns Shape European Attitudes Toward Asylum Seekers. Science, 354(6309), 217-222. doi: 10.1126/science.aag2147.

Behm-Morawitz, E., Pennell, H., \& Speno, A.G. (2016). The Effects of Virtual Racial Embodiment in a Gaming App on Reducing Prejudice. Communication Monographs, 83(3), 396-418. https://doi.org/10.1080/03637751.2015.1128556.

Bochner, A.P., Ellis, C., \& Tillman-Healy, L.M. (2000). Relationships as Stories: Accounts, Storied Lives, Evocative Narratives. In K. Dindia \& S. Duck (eds.), Communication and Personal Relationships (pp. 13-29). New York, NY: John Wiley \& Sons.

Brown, R., \& Hewstone, M. (2005). An Integrative Theory of Intergroup Contact. Advances in Experimental Social Psychology, 37, 255-343. https://doi.org/10.1016/ Soo65-2601(05)37005-5.

Cohen, J. (2001). Defining Identification: A Theoretical Look at The Identification of Audiences with Media Characters. Mass Communication \& Society, 4, 245-264. doi:10.1207/S15327825MCSo403_o1.

Daft, R.L., \& Lengel, R.H. (1984). Information Richness: A New Approach to Managerial Behavior and Organizational Design. In L.L. Cummings \& B.M. Staw (eds.), Research in Organizational Behavior, pp. 191-233. Homewood, IL: JAI Press.

Fisher, W.R. (1987). Human Communication as Narration: Toward a Philosophy of Reason, Value, and Action. Columbia, SC: University of South Carolina Press. 
Fuchs, L.H. (1990). The American Kaleidoscope: Race, Ethnicity, and the Civic Culture. Hanover, NH: Wesleyan University Press.

Gentile, D.A. et al. (2009). The Effects of Prosocial Video Games on Prosocial Behaviors: International Evidence from Correlational, Longitudinal, and Experimental Studies. Personality and Social Psychology Bulletin, 35, 752-763. doi: 10.1177/0146167209333045.

Gerrig, R.J. (1993). Experiencing Narrative Worlds. New Haven, CT: Yale University Press.

Green, M.C., \& Brock, T.C. (200o). The Role of Transportation in the Persuasiveness of Public Narratives. Journal of Personality and Social Psychology, 79, 701-721. doi:10.1037/0022-3514.79.5·701.

Greitemeyer, T., \& Mügge, D.O. (2014). Video Games do Affect Social Outcomes: A Meta-Analytic Review of the Effects of Violent and Prosocial Video Game Play. Personality and Social Psychology Bulletin, 4o(5), 578-589. doi:10.1177/0146167213520459.

Gutierrez, B., Kaatz, A., Chu, S., Ramirez, D., Samson-Samuel, C., \& Carnes, M. (2014). "Fair Play": A Videogame Designed to Address Implicit Race Bias Through Active Perspective Taking. Games for Health Journal, 3(6), 371-378. https://doi. org/10.1089/g4h.2013.0071.

Haddock, G., Zanna, M.P., \& Esses, V.M. (1993). Assessing the Structure of Prejudicial Attitudes: The Case of Attitudes Toward Homosexuals. Journal of Personality and Social Psychology, 65, 1105-1118. https://doi.org/10.1037/ 0022-3514.65.6.1105.

Harwood, J. (2010). The Contact Space: A Novel Framework for Intergroup Contact Research. Journal of Language and Social Psychology, 29, 147-177. 10.1177/0261927Xo9359520

Hodson, G., \& Hewstone, M. (eds.). (2013). Advances in Intergroup Contact. London: Psychology Press.

Janis, I.L., \& King, B.T. (1954). The Influence of Role Playing on Opinion Change. The Journal of Abnormal and Social Psychology, 49, 211-218. https://doi.org/10.1037/ hoo56957.

Klimmt, C. (2009). Serious games and social change: Why they (Should) Work. In U. Ritterfeld, M. Cody, \& P. Vorderer (eds.), Serious Games: Mechanisms and Effects (pp. 248-270). New York, NY: Routledge.

Klimmt, C., Hefner, D., \& Vorderer, P. (2009). The Video Game Experience as “True” Identification: A Theory of Enjoyable Alterations of Players' Self-Perception. Communication Theory, 19, 351-373. doi:10.1111/j.1468-2885.2009.01347.x.

Kumar, N., \& Benbasat, I. (2002). Para-Social Presence and Communication Capabilities of a Web Site: A Theoretical Perspective. E-Service Journal, 1, 5-24. doi:10.1353/esj.2002.0012. 
Lombard, M., \& Ditton, T. (1997). At the Heart of it All: The Concept of Presence. Journal of Computer-Mediated Communication, 3(2). https://doi. org/10.1111/j.1083-6101.1997.tbooo72.x.

___ et al. (200oh). Measuring Presence: A Literature-Based Approach to the Development of a Standardized Paper-And-Pencil Instrument. In Third International Workshop on Presence, Delft, the Netherlands, 240. Retrieved 7 July 2018 from http://citeseerx.ist.psu.edu/viewdoc/summary?doi=10.1.1.132.4737.

Neys, J., Van Looy, J., De Grove, F., \& Jansz, J. (2012). Poverty is Not a Game: Behavioral Changes and Long-Term Effects After Playing PING. Proceedings from The $62^{\text {nd }}$ Annual Conference of the International Communication Association. Phoenix, AR, USA. Retrieved 6 March 2018 from https://lib.ugent.be/nl/catalog/pugo1: 3103404 ? $\mathrm{i}=1 \& \mathrm{q}=$ Poverty+Is+Not+a+Game $\% 3 \mathrm{~A}+$ Behavioral + Changes+and+Lon g+Term+Effects+After+Playing+PING.

Pavlović, I., \& Marković, S. (2011). The Effect of Music Background on the Emotional Appraisal of Film Sequences. Psihologija, 44, 71-91. doi: 10.2298/PSI1101071P.

Peng, W. (2008). The Mediational Role of Identification in the Relationship Between Experience Mode and Self-Efficacy: Enactive Role-Playing Versus Passive Observation. Cyberpsychology \& Behavior, 11, 649-652. doi: 10.1089/cpb.2007.0229.

— Lee, M., \& Heeter, C. (2010). The Effects of a Serious Game on Role-Taking and Willingness to Help. Journal of Communication, 6o, 723-742. https://doi. org/10.1111/j.1460-2466.2010.01511.x.

Pettigrew, T.F. (1998). Intergroup Contact Theory. Annual Review of Psychology, 49, 65-85. https://doi.org/10.1146/annurev.psych.49.1.65

— , \& Tropp, L.R. (2006). A Meta-Analytic Test of Intergroup Contact Theory. Journal of Personality and Social Psychology, 9o, 751-783. doi:10.1037/0022-3514.90.5.751.

Ravaja, N., Salminen, M., Holopainen, J., Saari, T., Laarni, J., \& Järvinen, A. (2004). Emotional Response Patterns and Sense of Presence During Video Games: Potential Criterion Variables for Game Design. Proceedings from The Third Nordic Conference on Human-computer Interaction. Tampere, Finland, October 23-27, 2004. doi:10.1145/1028014.1028068.

Ruggiero, D. (2015). The Effect of a Persuasive Social Impact Game on Affective Learning and Attitude. Computers in Human Behavior, 45, 213-221. doi: 10.1016/j. chb.2014.11.062.

Schweitzer, R., Perkoulidis, S., Krome, S., Ludlow, C., \& Ryan, M. (2005). Attitudes Towards Refugees: The Dark Side of Prejudice in Australia. Australian Journal of Psychology, 57, 170-179. doi:10.1080/00049530500125199.

Stephan, W.G., \& Stephan, C.W. (200o). An Integrated Threat Theory of Prejudice. In S. Oskamp (Ed.), Reducing Prejudice and Discrimination (23-45), Mahwah, NJ: Lawrence Earlbaum Associates, Inc. 
Stephan, W.G., Ybarra, O., Martinez, C., Schwarzwald, J. \& Tur-Kaspa, M. (1998). Prejudice Toward Immigrants to Spain and Israel: An Integrated Threat Theory Analysis. Journal of Cross-Cultural Psychology, 29, 559-576. https://doi. org/10.1177/0022022198294004.

Tajfel, H. (1970). Experiments in Intergroup Discrimination. Scientific American, 223, 96-102. Retrieved 3 May 2018 from https://www.jstor.org/stable/2492766

— \& Turner, J.C. (1979). An Integrative Theory of Intergroup Conflict. In W. G. Austin, \& S. Worchel (eds.), The Social Psychology of Intergroup Relations, pp. 33-47. Monterey, CA: Brooks/Cole.

UNHCR (2006). Against All Odds. [Digital Game].

Van Looy, J., Courtois, C., De Vocht, M., \& De Marez, L. (2012). Player Identification in Online Games: Validation of a Scale for Measuring Identification in Mmogs. Media Psychology, 15(2), 197-221. doi:10.108o/15213269.2012.674917.

Van't Riet, J., Meeuwes, A.C., van der Voorden, L. \& Jansz, J. (2018) Investigating the Effects of a Persuasive Digital Game on Immersion, Identification, and Willingness to Help. Basic and Applied Social Psychology, 40(4), 180-194, doi: 10.1080/01973533.2018.1459301.

Walther, J.B. (1996). Computer-Mediated Communication: Impersonal, Interpersonal, and Hyperpersonal Interaction. Communication Research, 23, 3-43. https:// doi.org/10.1177/009365096023001001.

Watts, W.A. (1967). Relative Persistence of Opinion Change Induced by Active Compared to Passive Participation. Journal of Personality and Social Psychology, 5(1), 4-15. https://doi.org/10.1037/hoo21196.

\section{About the authors}

Chad Wertley is an Assistant Professor of Communication at Robert Morris University. His research focuses on how social identity influences communication within mediated contexts. Some of his notable research projects have explored the potential of video games as sites for positive intergroup contact and the use of communicative aggression to signal membership and status within a community.

Jordan Soliz is Professor of Intergroup and Family Communication at the University of Nebraska-Lincoln. His current projects focus on communication in multiethnic-racial families and interfaith families with a goal toward understanding communicative dynamics associated with individual well-being and relational-family solidarity. He also investigates outcomes of intergroup contact and intergroup dialogue that minimize negative outgroup attitudes (e.g., racism, ageism) and socialization processes. 


\title{
13. Mitigating Bias and Improving Professional Decision-Making through Persuasive Training Games
}

\author{
Yu-Hao Lee, Norah E. Dunbar, Claude H. Miller, Elena \\ Bessarabova, Matthew Jensen, Scott Wilson, Javier Elizondo, \\ Judee Burgoon, \& Joseph Valacich
}

\begin{abstract}
Making accurate, unbiased decisions is critical in high-stakes professions such as law enforcement, intelligence analysis, and medicine, since the decisions can have severe consequences. In this chapter, we discuss what makes persuasive games effective for training professionals to recognize their cognitive biases, improve their knowledge about decision-making biases, and learn ways of mitigating bias. We describe our experience designing three games for professional training in cognitive biases and deception detection. This chapter focuses on the combination of decisionmaking, education, and game theories that drives our design. This is then followed by a discussion of our experiments and measurements for testing the effectiveness of our designs.
\end{abstract}

Keywords: cognitive bias; decision-making; training; deception

\section{Introduction}

Many professions require the processing of information from multiple sources and the making of rapid decisions under pressure. Making accurate decisions is especially crucial in high-stakes settings such as law enforcement, intelligence analysis, and medicine. Since high-stakes decisions often have grave consequences involving life and death situations, one would expect professionals in these fields to make unbiased decisions by

Hera, T. Dela, J. Jansz, J. Raessens, B. Schouten, Persuasive Gaming in Context. Amsterdam: Amsterdam University Press, 2021 DOI 10.5117/9789463728805_CH13 
considering all available information, treating evidence fairly, and rationally weighing all the relevant costs and benefits. Research has shown, however, that due to the rapid-response nature of these kinds of judgments, decisionmakers often rely on heuristics, especially when in unfamiliar situations that require careful, deliberative processing (Hicks \& Kluemper, 2011; Heuer, 1999).

Heuristics are mental shortcuts, or rules of thumb, that simplify decisionmaking by reducing the amount of information required for processing (Tversky \& Kahneman, 1974; Chaiken, 1980). Most of the time, heuristics help professionals to make efficient judgments that are reasonably accurate. However, in unfamiliar situations, where careful, deliberative evaluations are required, relying on heuristics can lead to systematic cognitive biases and significant adverse consequences. Studies have shown, for example, how medical professionals often make premature diagnoses as a result of various cognitive biases (Graber, Franklin, \& Gordon, 2005). Bias can also cloud judges and jurors' memories of factual details during legal trials (Levinson, 2007).

Heuristics and cognitive biases are difficult to avoid because most people are unaware of their own biases (Pronin, Lin, \& Ross, 2002). Indeed, even when reminded and cautioned about their overuse of heuristics, people often lack the motivation to inhibit them due to the convenience they bring to the decision-making process (De Neys, Vartanian, \& Goel, 2008). Currently, organizational workshops and static, non-interactive instructional materials (e.g., videos and handouts) are the most common training formats for educating professionals about their cognitive biases. However, meta-analyses of studies on de-biasing training, especially in deception detection, indicate that the effects of traditional approaches are only small to moderate at best, depending on how the training is conducted (Driskell, 2012; Hauch et al., 2016). Decision-making training programs are more effective when they are relevant to real-world scenarios, teach diagnostic cues, allow the practicing of decision-making, and incorporate immediate feedback (Vrij, 2008; Frank \& Feeley, 2003).

We argue that digital games are effective training tools for mitigating bias and improving professional decision-making because of their ability to simulate real-world problems while providing tailored, individual feedback to players (Dunbar et al., 2018; Dunbar et al., 2014; Lee et al., 2016; Bessarabova et al., 2016). Below we list some reasons for adopting digital games in professional training.

(1) The simulation of real-life scenarios: Digital games can simulate highstakes decision scenarios without resulting in actual life-and-death consequences. Games also allow for the simulation of rare or expensive scenarios, 
so that professionals can practice making decisions under extreme and challenging conditions. It would be very costly and dangerous for surgeons to practice on actual patients during early training or for law-enforcement recruits to engage with dangerous criminals. A game simulation, however, can offer such challenges within a safe and controlled environment.

(2) Systemic thinking: Digital games are especially good at facilitating complex and dynamic systems-thinking (Squire, 2006). Games allow players to build mental models of interacting factors by visualizing the variables, manipulating the factors, and observing the changes. Thus, a game like SimCity allows players to learn the relationship between budgets and policies and to understand how each decision can cause a chain reaction that affects further outcomes based on players' decisions.

(3) Personal feedback: Digital games can give immediate feedback to players about their performance and decisions (Azevedo \& Bernard, 1995). They can also provide formative, corrective feedback following key decisions that are taken or not taken. This gives players information about potential outcomes, enabling them to reconsider their decisions and make adjustments within a dynamic, ongoing, interactive process.

(4) Autonomy: While not all persuasive games are played voluntarily, almost all of them involve choice and provide some level of autonomy and agency for players (Ryan, Rigby, \& Przybylski, 2006). The freedom to make choices that have an effect on the environment-and on others with whom one is interacting within a video game - can be a powerful source of enjoyment (Klimmt, Hartmann, \& Frey, 2007), further stimulating intrinsic motivation.

(5) Community-building: Many approaches to training involve teambuilding games to facilitate trust, foster cohesion, and create a community of like-minded individuals sharing similar goals. Digital games can create strong senses of community and identity, with people coming together to discuss issues and problems experienced within a game (Gee, 2007). Games are fundamentally creative problems to be solved. To unravel and resolve in-game problems, players work together to actively process information in the simulated environment, learn about its rules, and pick up on relevant skills as they progress.

Overall, the procedural nature of digital games allows immediate and individualized corrective feedback about a player's progress and performance (Bogost, 2007), providing an opportunity to assess and alter decisions (Billings, 2010). Moreover, because games incorporate designs that support and encourage self-determination-such as competence, autonomy, and relatedness - they tend to be more intrinsically motivating, 
resulting in the greater internalization of relevant training materials (Tamborini et al., 2010; Ryan, Rigby, \& Przybylski, 2006). Taken together, the features of persuasive games offer the potential for bias mitigation and decision-making training.

Based on our team's experience of designing and testing three different persuasive games developed for the law enforcement and intelligence communities, this chapter focuses on the benefits of using digital games to train professionals to avoid biases and heuristics in order to improve their decision-making. We start the chapter with a description of the games that our teams developed, followed by a discussion of the measurements and theory-driven designs that made the exploration of the effects of persuasive game possible. We conclude with a description of our research findings that together point to the effectiveness of employing persuasive digital games for bias mitigation and deception-detection training.

\section{Persuasive games}

\section{MACBETH}

The first bias persuasive training game we developed was called MACBETH (Mitigating Analyst Cognitive Bias by Eliminating Task Heuristics) and was funded by the Sirius game program of IARPA (Intelligence Advanced Research Projects Activity). The goals of the research were to test the effectiveness of digital games for teaching intelligence analysts about cognitive biases and to improve their decision-making processes. MACBETH addressed three types of bias: confirmation bias (i.e., the tendency to select or interpret information that confirms one's values, attitudes, or beliefs); fundamental attribution error (i.e., the tendency to attribute other people's behavior to dispositions and to underemphasize situational factors); and bias blind spot (i.e., the inability to see one's own biases while being aware of those in others).

MACBETH was designed as a turn-based strategy game in which players take on the role of an intelligence analyst attempting to prevent a potential terrorist attack. The nine scenario narratives and game contexts were designed to mirror the problems that intelligence analysts face in real life and to increase skill transfer from the game to their actual work. In the game, a short description of cognitive biases was provided in the introduction. To prevent a terrorist attack, players had to gather intelligence from multiple sources to generate their hypotheses about 


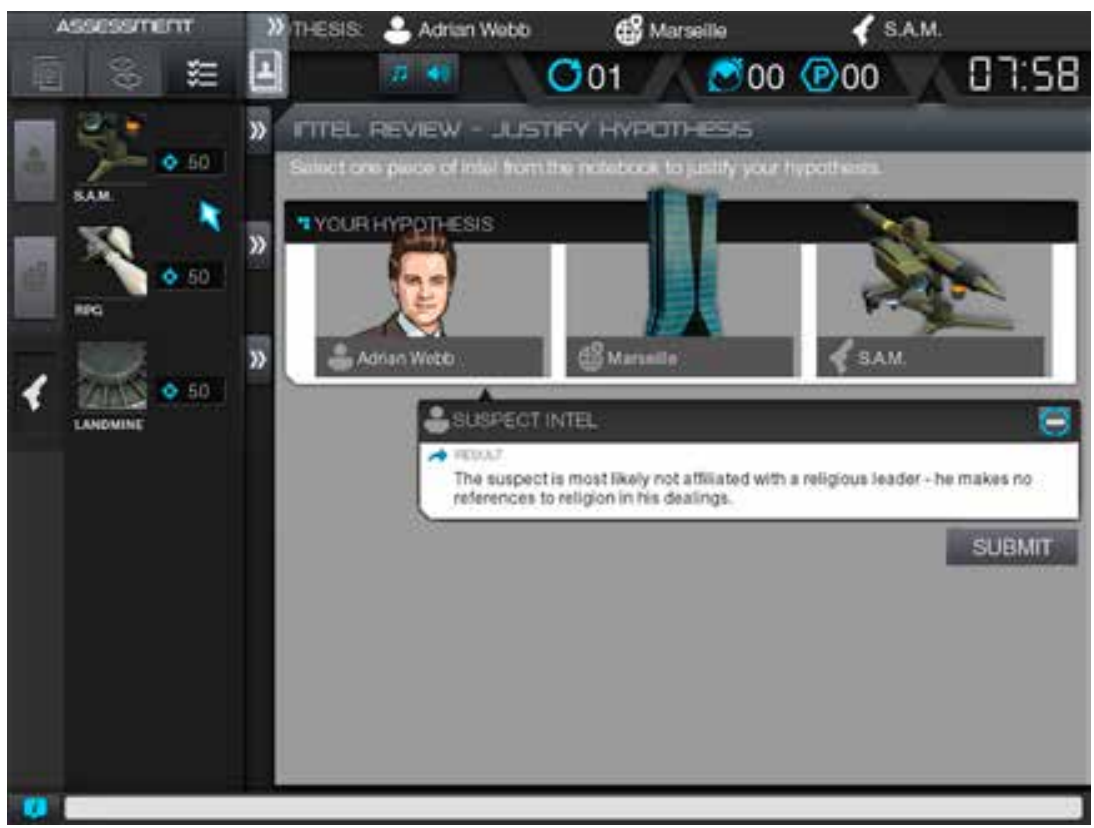

Figure 13.1

the suspect, the location, and the means of the attack. To win the game, players had to distinguish between biased and unbiased intelligence and rely on the latter to make their decisions. See Figure 13.1 for a screenshot of MACBETH.

To reduce confirmation bias, MACBETH provided players with feedback encouraging them to delay forming hypotheses while searching for more disconfirming information that would be helpful for formulating alternative hypotheses. To reduce fundamental attribution errors, a mini-game was developed wherein players read through old case files that described historical figures, some of whom were actual criminals and terrorists. To succeed, players needed to select information that was diagnostic when it came to correctly identifying historical characters as threats, with points awarded for choosing situational clues over dispositional versions (see Dunbar et al., 2014 for more details). To reduce bias blind spots, the goal was to demonstrate to players that they are just as susceptible to bias as everyone else. The presence of bias was communicated both implicitly by taking points off for biased decisions (and in some cases through losing a particular level within the game) and more explicitly by giving players feedback about the different types of bias they exhibited as they occurred (see Bessarabova et al., 2016 for more details). 


\section{MACBETH 2}

The second game, MACBETH 2 (also funded by IARPA, with supplemental funding from the University of Oklahoma), was designed to address three different types of cognitive bias: anchoring bias (i.e., the tendency to overemphasize the first piece of information received by anchoring subsequent judgments to it, leading to overestimates or underestimates); projection bias (i.e., the tendency to project oneself onto others when making judgments); and representativeness bias (i.e., the tendency to ignore statistical probabilities in favor of contextual cues). Representative bias can be further divided into four subtypes: stereotyping (i.e., overgeneralizing attributes based on group characteristics); insensitivity to sample size (i.e., overgeneralization from small samples); base-rate fallacy (i.e., disregarding the probability of outcomes and focusing on descriptive information to estimate probabilities); and gambler's fallacy (i.e., the misconception of chance, or the tendency to expect a sequence of random events to be non-random, such as when expecting a higher chance of winning after a series of losses).

In an attempt to counteract these biases, the design of MACBETH 2 was similar to MACBETH but with a few changes. First, players were tasked with instructing a field agent to move around in a virtual environment to gather information about a series of international contraband trafficking cases. Next, players were asked to determine if the information the agent gathered was biased or not. In addition to making judgments about the information, players also had to identify which kind of bias was involved in the field agent's various decisions. Once a certain amount of information was collected, the player took the intelligence into consideration and assessed whether the suspect being investigated in each scenario was a threat or not. See Figure 13.2 for a screenshot of MACBETH 2.

We used a multiple-choice question format for anchoring bias mitigation. In the game, players were exposed to the field agent's estimates (i.e., an anchor) that were unrelated to the decision being made, and they needed to produce an estimate without being influenced by the irrelevant anchor. To facilitate this, we used a consider-the-opposite strategy developed by Mussweiler, Strack, and Pfeiffer (2000) to train players on anchoring bias. Before the players made the estimate, they received a multiple-choice question, providing one answer in which the anchor was relevant to the estimate and three suggesting that the anchor was irrelevant to the targeted estimate. The goal was to prompt players to consider alternative explanations and separate the anchor from their estimates. 


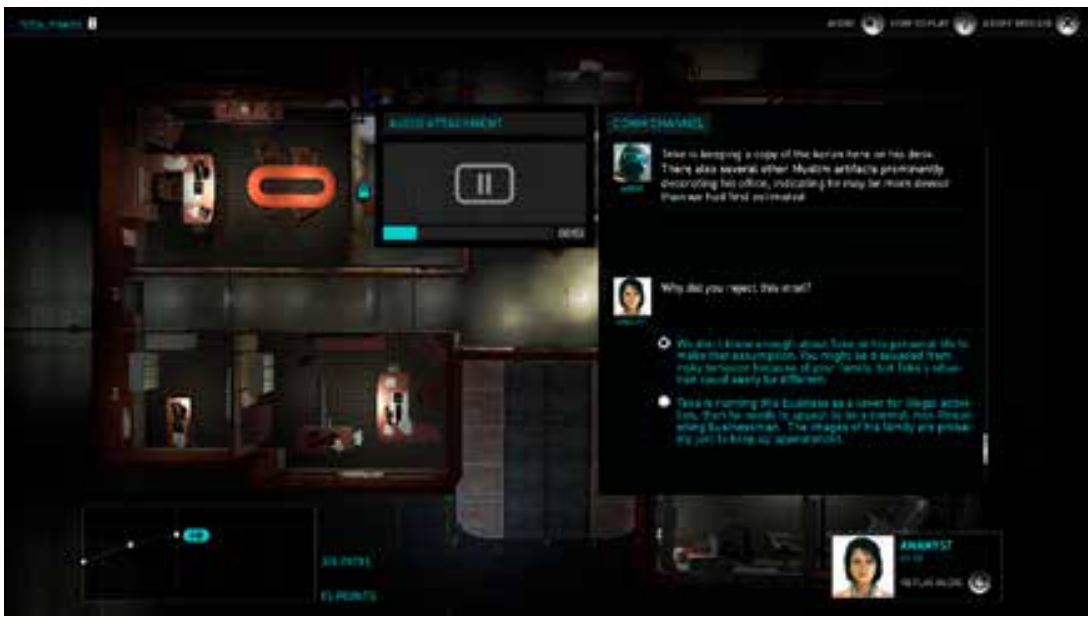

Figure 13.2

We employed two separate strategies to mitigate representativeness bias. The first addressed stereotyping and base-rate fallacy. The game asked players to read feedback about biased intelligence in which various descriptions were included along with base-rate statistics relevant to how the information might be biased. The players were encouraged to take the base rate into consideration instead of relying solely on the representative descriptions. The second strategy addressed the gambler's fallacy and insensitivity to sample size. The game provided players with two similar examples of a situation, after which the field agent offered his or her assessment of the pattern observed from the previous scenarios. Players could choose to reject the agent's assessment based on the small sample sizes observed, thereby considering chance rates instead of the proffered patterns (see Lee et al., 2016 for more details).

\section{VERITAS}

Our third game for professional training is called VERITAS (Veracity Education and Reactance Instruction through Technology and Applied Skills) and was funded by a grant from the National Science Foundation's Cyberlearning and Future Learning Technologies program. The goal was to design and test a game to teach deception-detection skills to law enforcement officers. In real-world decision-making, deception is very difficult to detect, and professionals are known to rely on stereotypes and biases that are not based on reliable cues (Vrij, 2008). We trained players to identify clusters of verbal and non-verbal cues that indicated tension, uncertainty, and the 


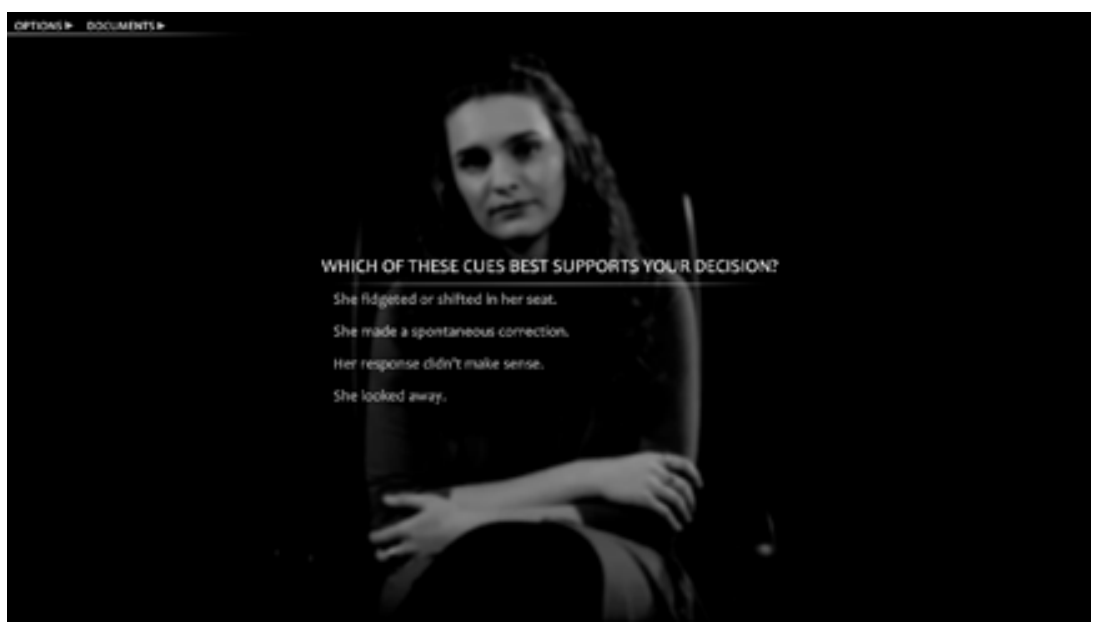

Figure 13.3

high cognitive load that is more likely to be associated with deception than truthfulness (Dunbar et al., 2018).

In VERITAS, two actors played characters in two game scenarios: a job interview and a workplace theft investigation. The purpose of using real actors rather than computer-generated characters was to enhance the training effects by more accurately depicting the verbal and non-verbal truth and deception cues while avoiding uncanny valley effects (i.e., a dip in the player's affinity for a computer-generated character due to its eeriness). In the game, players take on the role of the interviewer and select questions to ask the interviewees from a series of options. See Figure 13.3 for a screenshot of VERITAS.

The game reacts to player questions using a dialog tree that answers with pre-recorded responses performed by the actors. For each answer, players are asked to assess whether the statement is truthful or deceptive and to report their degree of confidence in each judgment. The players must also indicate which cues they relied on to form their judgments. The training begins with a brief instructional video in the game that teaches players what cues to look for instead of relying on stereotypes or myths about deception. The first half of Scenario 1 serves as a baseline assessment of the player's deception-detection skills. The player is given performance feedback midway 
through this scenario. Afterwards, the players receive formative feedback about their judgments, explaining why they were correct or incorrect and what cues they should have focused on or ignored.

\section{Theory-driven design and measurements}

A key to designing games for professional training is to ensure that the learning effects can transfer to real-world scenarios and last beyond the immediate training session. Other considerations involve testing whether the game can be as effective as traditional instructional methods like lectures and videos. To increase intrinsic motivation within the game, it is also important to determine how best to reduce possible negative reactions (i.e., psychological reactance) that arise due to potential controlling influences within the training environment. Theories of learning, bias mitigation, and social influence were incorporated into each game to achieve these goals. These theory-based approaches are detailed further below.

To improve knowledge and skill transfer, early theories of learning highlight the importance of including common features within the training to facilitate transfer by helping learners make associations between the features (Thorndike \& Woodworth, 1901). More recent theories regarding skill learning argue that training mechanics should help learners to build mental models that are transferable to real-life scenarios. The training should also feature a certain amount of variability so that pertinent knowledge and skills are not tied to specific scenarios but can be generalized to new and changing circumstances (Burke \& Hutchins, 2007). We designed our games' contexts based on these concepts in order to mirror the challenges within real-life scenarios faced by professionals each day. In MACBETH and MACBETH 2, players adopt the role of an intelligence analyst, whereas in VERITAS they assume the role of an interviewer evaluating an interviewee or a potential suspect in a crime. In terms of game mechanics, since the focus is on decisionmaking rather than navigating virtual environments, a turn-based puzzle/ strategy format was chosen so that players could more carefully focus on managing a significant amount of information during the decision-making process. In all three games, multiple scenarios were designed to vary in terms of their context and the way various forms of information (including biased information) were presented. The variability helps players to learn not to over-rely on one piece of information but to construct a general mental model about the biases and deception that considers as much of the information and cues as possible in relation to each other. 
We embedded behavioral measures in all three of our games to assess short- and long-term effects and to identify progress during the learning process. Behavioral measures such as how many times the players performed a particular action, the sequence of their actions, and the duration of activities allowed us to track how players were interacting within the games and what choices they made. These data gave us a better understanding of which features were used more often and which were under-utilized. The behavioral data also allowed us to track player performance unobtrusively, to see if players were gradually improving during the training process, and to determine how long they spent in the game.

To assess the immediate and long-term effects of the games, we developed measurements of each bias based on previous literature. We administered the tests immediately after our study participants played the game and then again four to eight weeks later. As one of the goals was to measure transfer, the measurements were designed to be in a format that differed from the one used in the game. Thus, the players in MACBETH 2 learned how to avoid anchoring bias by considering alternative explanations and were prompted by the game to consider the possibility that the anchor was irrelevant to the target estimate. Meanwhile, for the post-game measures, and following experimental manipulations by Tversky and Kahneman (1974), we described an irrelevant number such as 'the tallest bridge in the United Kingdom suspends 2,431 feet above the ground' and asked participants to estimate how many candies there were in a picture of a candy jar (see Adame, 2016 for more details). In other words, the immediate and long-term measures did not share the same format as the training, enabling us to capture how well players can transfer the skills learned in the game to a similar context.

The third goal of our studies was to determine how the effects of the digital game training compared to traditional instructional videos. For the MACBETH studies, the comparison video was provided by the project's funding agency. The instructional video featured a series of short skits followed by an instructor figure explaining the types of bias displayed in them and providing tips on how to avoid them. This was produced independently from the design and content that we developed for our game. Although we were required by the funder to use the instructional video as a comparison group, we were concerned that the instructional content in the game and the video was not identical, which made it difficult to ascertain whether our results were due to differences in the medium (video vs. video game) or in the educational content. Nonetheless, even with this limitation, our results indicate the superiority of the long-term effects of a video game over an instructional video. 
To increase correspondence between the game and the comparison group, we created our comparison videos in the MACBETH 2 and VERITAS projects from an animated PowerPoint lecture for the experiments. The instructional video content and de-biasing instructions were created to match the same content and instructions taught in the game. By comparing the game to an instructional video with similar training content, we were able to identify media features and mechanics that made video-game-based training more effective than an instructional video.

The final issue in need of resolution involved obviating the adverse psychological effects associated with resistance to training. As people are unaware of their own biases (Pronin, Lin, \& Ross, 2002), convincing professionals they are just as susceptible to bias as everyone else requires careful message design to reduce their reactance to training while also motivating them to play the game. Our reasoning is grounded in psychological reactance theory, which posits that explicit persuasive messages can pose threats to the message recipients' perceived behavioral freedoms, resulting in reactance, wherein they seek to reestablish their autonomy by rejecting the message, derogating the source, and exhibiting behaviors in the direction opposite to those advocated (i.e., boomerang effects; Brehm \& Brehm, 1981). We tested different levels of explicit (controlling) vs. implicit (autonomy-supportive) language within various aspects of the game to avoid reactance while also balancing the effectiveness of the games' instructions.

\section{Summary of findings}

We conducted eight studies with more than 2,00o participants between 2013 and 2017 across four universities in the United States (University of Oklahoma, University of Arizona, University of California-Santa Barbara, and University of Florida). This summary describes the factors we examined and their effectiveness in increasing bias-relevant knowledge and bias mitigation.

\section{Feedback timing and source}

One of the unique affordances of digital game-based learning is that digital games can provide feedback to players based on their performances. Many studies have examined the role of feedback in learning (e.g., Kluger \& DeNisi, 1996; Goldberg \& Cannon-Bowers, 2015; Shute, 2008; Hattie \& Timperley, 2007). We assessed the effects of feedback timing (immediate vs. delayed) and 
feedback source (non-player character vs. other players) on bias knowledge and bias mitigation.

Studies have shown that while providing immediate feedback during game-play can increase its salience and allow players to adjust their decisions, it can also disrupt the game and break the flow (Attali \& van der Kleij, 2017). As for the feedback source, studies have shown that explaining one's reasoning to someone else can reduce bias (Green, 1990); sometimes having other people question one's decision-making process can also make people more aware of their biases (Silverman, 1992).

We therefore designed two versions of MACBETH. In the single-player version, the players received feedback from computer-controlled non-player characters, whereas in the multi-player game, the players formed their judgments by working with another player and examining each other's proffered hypotheses. To our surprise, relative to the multi-player version, the single-player game in which players received feedback from a non-player (AI) character was significantly more effective at mitigating confirmation bias. This may be because playing with another player slowed down the pace of the game, and waiting for other players to respond interrupted the natural flow (see Dunbar et al., 2017, for more details). However, no significant differences between a single-player and a multi-player game were found for the knowledge or mitigation of fundamental attribution errors (Dunbar et al., 2017) or bias blind spots (Bessarabova et al., 2016).

One of our central hypotheses posited that providing players with immediate feedback would be more effective in mitigating bias and increasing knowledge about the issue than delayed feedback, presumably because immediate feedback enables players to think about their decisions right after they are made, allowing for immediate adjustments to their actions. However, there were no significant differences in bias mitigation between immediate versus delayed feedback (Dunbar et al., 2014; Bessarabova et al., 2016); in fact, our qualitative interviews suggested that some players found the immediate feedback annoying and perceived it as an interruption of their game-play.

\section{Repetition and duration}

An intrinsically motivating digital game is likely to be played for a longer duration, engaging players in the training materials for extended periods of time. Digital games can also be played repeatedly, which is important for experiential learning, as it gives learners frequent opportunities to experiment with different choices and compare the outcomes of their 
decisions (Kolb \& Kolb, 2005). We compared different durations (30 minutes vs. 60 minutes) of game-play and repetition (once vs. multiple times) for all of our studies involving cognitive biases.

Across our studies (with a few exceptions), we found that playing for a longer duration led to greater bias mitigation, more improved bias knowledge, and greater deception-detection skills. Repetition also significantly improved the effects of training. Increased duration and repetition had the effect of increasing exposure to the bias-related and deception-detection knowledge, providing players with more opportunities to practice their bias-mitigation and deception-detection skills. More importantly, repeated play is an essential requirement for a game-based training platform, given that learning game controls and often complex game mechanics is a prerequisite for engaging in the training; it is only after players learn to operate and navigate a game that cognitive resources can be freed up to focus on the training content presented (Lee \& Heeter, 2017).

\section{Implicit vs. explicit training}

In comparison to classroom learning, which is often explicit in its presentation of material to learners, digital games can incorporate implicit instructions by demonstrating the interworking of a complex system through gameplay (Bogost, 2007; Ciavarro, Dobson, \& Goodman, 2008). We created two versions of MACBETH to compare the differences between implicit and explicit video-game learning. In the explicit training condition, learning was attained through quizzes that appear throughout the game, testing players about their knowledge of biases. The implicit version did not have quizzes; instead, learning was embedded in the game mechanics and feedback.

Overall, our findings suggest that the implicit and explicit training conditions did not differ significantly in terms of effectiveness. However, the explicit training did increase bias knowledge (confirmation bias, bias blind spots, and fundamental attribution errors) immediately after playing the game, although these effects tended to fade over time (Dunbar et al., 2014).

\section{Instructional video comparison}

Interactive digital games have several advantages over traditional training videos. For the most part, the former allow players to actively make decisions and observe the immediate consequences of their actions, which is especially important when the training goal is to foster awareness of one's biases and modify decision-making processes accordingly. Video 
games can also provide a variety of simulated situations for players to practice their decision-making skills using multiple scenarios to promote skill transfer.

As mentioned above, in order to capture the differences between various educational methods, most of our experiments compared the effects of the game to an instructional video. Across the studies, we found our digital games were equally or more effective than the instructional videos in increasing bias knowledge and reducing biased decisions immediately after training. However, the effects of the instructional video quickly diminished, whereas the effects of the games did not, even after four to eight weeks. Players also reported higher enjoyment, motivation, and cognitive absorption while playing the games compared to watching instruction videos (Dunbar et al., 2014, 2017; Lee et al., 2016). The fact that the bias mitigation effects did not fade after eight weeks is remarkable, indicating that video games can be effective in the long term for decision-making training.

\section{Reactance to training}

When people feel like someone is trying to restrict their behavioral freedom or threaten their self-identity, they may react negatively and try to restore their threatened freedom (Brehm \& Brehm, 1981). We compared the use of controlling language to autonomy-supportive language within our VERITAS experiments. Surprisingly, the manipulations did not appear to make a significant difference for the undergraduate student sample (Dunbar et al., 2018). However, the effects of reactance were more pronounced and significant for the law enforcement officer sample. The game-based training was more effective on police officers when the game used autonomysupportive language within the instruction compared to more controlling language (Miller et al., 2018). One explanation for the difference between the students' responses and the law enforcement officers' responses may be because the latter group is not used to taking orders from university researchers. Another explanation is that, unlike police officers, students may not perceive themselves to be experts in detecting deception. In contrast, police officers may find their self-perceived authority threatened and could experience psychological reactance when the game explicitly instructs them to engage in deception training. If this is the case, they should be likely to respond more favorably toward autonomy-supportive instructions that frame the training as a way of improving their deceptiondetection skills. 


\section{Conclusion}

To persuade is to modify or reinforce people's attitudes, beliefs, and behaviors. Although training games are not always designed with persuasion in mind, we argue that most such games contain elements of social influence and persuasion. By offering players an opportunity to observe and examine their personal knowledge and skills, digital training games create the potential for self-persuasion (Zimbardo 1965; Bem, 1965; Aronson, 1999). In this way, digital games can expose gaps in one's understanding of a subject or skills within a non-threatening environment, thereby creating cognitive dissonance and uncertainty that can motivate players to investigate further, practice more, and improve their relevant skill sets. Such an approach might offer exceptionally strong motivation for professionals who may be overconfident in their ability to perform their work and thus undermotivated to receive further training. Studies have shown that roleplaying and perspective-taking can be effective for self-persuasion because they encourage players to actively come up with arguments in support of certain positions. As such arguments are self-generated, the effects of self-persuasion may thus be stronger and longer-lasting (Zimbardo, 1965; Peng, Lee, \& Heeter, 2010).

Our research shows that persuasive digital games offer an effective means for training bias mitigation, deception-detection, and improved decision-making. The interactive digital games that we tested consistently outperformed the more static instructional videos in terms of learning, enjoyment, motivation, and engagement. Of particular interest is the finding that the bias-mitigation effects of these serious digital games lasted longer than the comparison instructional videos. Informed by theories of psychology and social influence, the digital games reviewed here applied an iterative design approach that, through empirical evidence, helped to identify which methods were effective and which needed modification and improvement. Future persuasive game designs can benefit from this approach of incorporating theory into game design and using behavioral data and user feedback to improve the effects of the games.

\section{References}

Adame, B.J. (2016). Training in the Mitigation of Anchoring Bias: A Test of the Consider-The-Opposite Strategy. Learning and Motivation, 53, 36-48. https://doi. org/10.1016/j.lmot.2015.11.002. 
Aronson, E. (1999). The Power of Self-Persuasion. American Psychologist, 54(11), 875-884. https://doi.org/10.1037/hoo88188.

Attali, Y., \& van der Kleij. F. (2017). Effects of Feedback Elaboration and Feedback Timing During Computer-Based Practice in Mathematics Problem Solving. Computers \& Education, 110, 154-169. https://doi.org/10.1016/j.compedu.2017.03.012. Azevedo, R., \& Bernard, R.M. (1995). A Meta-Analysis of the Effects of Feedback in Computer-Based Instruction. Journal of Educational Computing Research, 13(2), 111-127. doi:10.3102/0034654314564881.

Bem, D.J. (1965). An Experimental Analysis of Self-Persuasion.Journal of Experimental Social Psychology, 1(3), 199-218. https://doi.org/10.1016/o022-1031(65)90026-o. Bessarabova, E. et al. (2016). Mitigating Bias Blind Spot Via a Serious Video Game. Computers in Human Behavior, 62, 452-466. https://doi.org/10.1016/j.chb.2016.03.089. Billings, D.R. (2010). Adaptive Feedback In Simulation-Based Training [Unpublished Doctoral Dissertation], University of Central Florida, Orlando, Florida.

Bogost, I. (2007). Persuasive Games: The Expressive Power of Videogames. Cambridge, MA: MIT Press.

Brehm, S.S., \& Brehm, J.W. (1981). Psychological Reactance: A Theory of Freedom and Control. New York, NY: Academic Press.

Burke, L.A., \& Hutchins, H.M. (2007). Training Transfer: An Integrative Literature Review. Human Resource Development Review, 6(3), 263-296. https://doi. org/10.1177/1534484307303035.

Chaiken, S. (1980). Heuristic Versus Systematic Information Processing and the Use of Source Versus Message Cues in Persuasion. Journal of Personality and Social Psychology, 39(5), 752-766. https://doi.org/10.1037/0022-3514.39·5·752.

Ciavarro, C., Dobson, M., \& Goodman, D. (2008). Implicit Learning as a Design Strategy for Learning Games. Computers in Human Behavior, 24(6), 2862-2872. doi:10.1016/j.chb.2008.04.011.

De Neys, W., Vartanian, O., \& Goel, V.. (2008). Smarter Than We Think When Our Brains Detect that We are Biased. Psychological Science, 19(5), 483-489. https:// doi.org/10.1111/j.1467-9280.2008.02113.x.

Driskell, J.E. (2012). Effectiveness of Deception Detection Training: A Meta-Analysis. Psychology, Crime \& Law, 18(8), 713-731. doi:10.1080/1068316X.2010.535820.

Dunbar, N.E., Jensen, M.L., Miller, C.H., Bessarabova, E., Lee, Y.H., Wilson, S.N., Elizondo, J., Adame, B.J., Valacich, J., \& Straub, S. (2017). Mitigation of Cognitive Bias with a Serious Game: Two Experiments Testing Feedback Timing and Source. International Journal of Game-Based Learning (IJGBL) 7(4), 86-100. doi:10.4018/ ijgbl.2017100105.

- et al. (2014). Implicit and Explicit Training in the Mitigation of Cognitive Bias Through the Use of a Serious Game. Computers in Human Behavior, 37, 307-318. https://doi.org/10.1016/j.chb.2014.04.053. 
et al. (2018). Reliable Deception Cues Training in an Interactive Video Game. Computers in Human Behavior, 85, 74-85. https://doi.org/10.1016/j.chb.2018.03.027 Frank, M.G., \& Feeley, T.H. (2003). To Catch a Liar: Challenges for Research in Lie Detection Training. Journal of Applied Communication Research, 31(1), 58-75. https://doi.org/10.1080/00909880305377.

Gee, J.P. (2007). What Video Games Have to Teach Us About Learning and Literacy. London: Macmillan Publishers.

Goldberg, B., \& Cannon-Bowers, J. (2015). Feedback Source Modality Effects on Training Outcomes in a Serious Game: Pedagogical Agents Make a Difference. Computers in Human Behavior, 52, 1-11. https://doi.org/10.1016/j.chb.2015.05.008. Graber, M.L., Franklin, N., \& Gordon, R. (2005). Diagnostic Error in Internal Medicine. Archives of Internal Medicine, 165(13), 1493-1499. doi:10.1001/archinte.165.13.1493. Green, D.W. (1990). Confirmation Bias, Problem-Solving and Cognitive Models. Advances in Psychology, 68, 553-562. https://doi.org/10.1016/So166-4115(o8)61342-4. Hattie, J., \& Timperley, H. (2007). The Power of Feedback. Review of Educational Research, 77(1), 81-112. https://doi.org/10.3102/003465430298487.

Hauch, V., Sporer, S.L., S Michael, S.W., \& Meissner, C.A. (2016). Does Training Improve the Detection of Deception? A Meta-Analysis. Communication Research 43(3), 283-343. doi:10.1177/0093650214534974.

Heuer, R.J. (1999). Psychology of Intelligence Analysis. Washington, DC: United States Government Printing Office.

Hicks, E.P., \& Kluemper, G.T. (2011). Heuristic Reasoning and Cognitive Biases: Are They Hindrances to Judgments and Decision Making in Orthodontics?. American Journal of Orthodontics and Dentofacial Orthopedics, 139(3), 297-304. https://doi.org/10.1016/j.ajodo.2010.05.018.

Klimmt, C., Hartmann, T., \& Frey, A. (2007). Effectance and Control as Determinants of Video Game Enjoyment. Cyberpsychology \& Behavior, $10(6), 845-848$. https:// doi.org/10.1089/cpb.2007.9942.

Kluger, A.N., \& DeNisi, A. (1996). The effects of Feedback Interventions on Performance: A Historical Review, a Meta-Analysis, and a Preliminary Feedback Intervention Theory. PsychologicalBulletin, 119(2), 254-284. https://doi.org/10.1037/0033-2909.119.2.254.

Kolb, A.Y., \& Kolb, D.A. (2005). Learning Styles and Learning Spaces: Enhancing Experiential Learning in Higher Education. Academy of management learning \& education, 4(2), 193-212. https://doi.org/10.5465/amle.2005.17268566.

Lee, Y.H. et al. (2016). Training Anchoring and Representativeness Bias Mitigation Through a Digital Game. Simulation \& Gaming, 47(6), 751-779. https://doi. org/10.1177/1046878116662955.

, \& Heeter, C. (2017). The Effects of Cognitive Capacity and Gaming Expertise on Attention and Comprehension. Journal of Computer Assisted Learning 33 (5):473-485. doi: 10.1111/jcal.12193. 
Levinson, J.D. (2007). Forgotten Racial Equality: Implicit Bias, Decision-Making and Misremembering. Duke Law Journal, 57(2), 345-424. Retrieved 11 June 2019 https://papers.ssrn.com/sol3/papers.cfm?abstract_id=927547.

Miller, C.H. et al. (2018). Training Law Enforcement Officers to Identify Reliable Deception Cues with an Interactive Digital Game. The 51st Hawaii International Conference on System Sciences, Kona, Hawaii.

Mussweiler, T., Strack, F., \& Pfeiffer, T. (2000). Overcoming the Inevitable Anchoring Effect: Considering the Opposite Compensates for Selective Accessibility. Personality and Social Psychology Bulletin, 26(9), 1142-115o. https://doi. org/10.1177/01461672002611010.

Peng, W., Lee, M., \& Heeter, C. (2010). The Effects of a Serious Game on Role-Taking and Willingness to Help.Journal of Communication 6o(4), 723-742. https://doi. org/10.1111/j.1460-2466.2010.01511.x.

Pronin, E., Lin, D., \& Ross, L. (2002). The Bias Blind Spot: Perceptions of Bias in Self Versus Others. Personality and Social Psychology Bulletin, 28(3), 369-381. doi: 10.1177/0146167202286008.

Ryan, R.M., Rigby, C.S., \& Przybylski, A. (2006). The Motivational Pull of Video Games: A Self-Determination Theory Approach. Motivation and Emotion, 30(4), 344-36o. https://doi.org/10.1007/s11031-006-9051-8.

Shute, V.J. (2008). Focus on Formative Feedback. Review of Educational Research, 78(1), 153-189. https://doi.org/10.3102/0034654307313795.

Silverman, B.G. (1992). Modeling and Critiquing The Confirmation Bias In Human Reasoning. IEEE Transactions on Systems, Man and Cybernetics, 22(5), 972-982. doi:10.1109/21.179837.

Squire, K. (2006). From Content to Context: Videogames as Designed Experience. Educational Researcher, 35(8), 19-29. https://doi.org/10.3102/ o013189X035008019.

Tamborini, R., Bowman, N.D., Eden, A., Grizzard, M., \& Organ, A. (2010). Defining Media Enjoyment as the Satisfaction of Intrinsic Needs. Journal of communication, 6o(4), 758-777. https://doi.org/10.1111/j.146o-2466.2010.01513.x.

Thorndike, E.L., \& Woodworth, R.S. (1901). The Influence of Improvement in One Mental Function Upon the Efficiency Of Other Functions. Psychological review, 8(3), 247- 261. https://doi.org/10.1037/hoo74898.

Tversky, A., \& Kahneman, D. (1974). Judgment under Uncertainty: Heuristics and Biases. Science, 185(4157), 1124-1131. https://doi.org/10.1126/science.185.4157.1124. Vrij, A. (2008). Detecting Lies and Deceit: Pitfalls and Opportunities. Hoboken, NJ: John Wiley \& Sons.

Zimbardo, P.G. (1965). The Effect of Effort and Improvisation on Self-Persuasion Produced by Role-Playing. Journal of Experimental Social Psychology, 1(2), 103-120. https://doi.org/10.1016/0022-1031(65)90039-9. 


\section{About the authors}

Yu-Hao Lee's research focuses on information processing in interactive and immersive media such as video games, virtual reality, and augmented reality. He has been involved in several design-based research projects that incorporate psychology theories to promote better decision-making and engagement funded by the National Science Foundation, the UNHCR, the IARPA, and the Online News Association.

Norah E. Dunbar is Professor of Communication at UC Santa Barbara. She has received over $\$ 13$ million in research funding from agencies such as the Intelligence Advanced Research Projects Activity and the National Science Foundation. She has published over 65 peer-reviewed journal articles and book chapters and has presented over 100 papers at national and international conferences.

Claude H. Miller is Professor and Director of Graduate Studies at the University of Oklahoma. Dr. Miller's work investigates human affective responses to influence messages in various contexts by applying emotion, motivation, and social influence theories, particularly to mass-mediated message designs targeting adolescent, elderly, and minority populations.

Elena Bessarabova is Associate Professor at the University of Oklahoma. Her research focus is in the areas of social influence and cross-cultural communication. Within social influence, she is interested in resistance processes. Specifically, Bessarabova studies resistance processes associated with psychological reactance, inoculation, and bias mitigation.

Matthew Jensen is Associate Professor of Management Information Systems at the University of Oklahoma. His research interests include computer-aided decision-making, knowledge management, human-computer interaction, and computer-mediated communication. He is an active member of an interdisciplinary team investigating how people attribute credibility in mediated interactions and how people filter and evaluate information they find online.

Scott Wilson is Director for Innovative Learning at the K2o Center for Educational and Community Renewal at the University of Oklahoma. Scott coordinates a number of research, development, and technical assistance teams that support students, teachers, and learning communities as they 
work to access learning tools, resources, and supports that help learners realize their full potential.

Javier Elizondo works at the K2o Center at the University of Oklahoma as an educational video game producer where in collaboration with an extraordinary team of game developers, instructional designers, and subject matter experts he has participated in the creation of 14 games in the last six years.

Judee Burgoon is Professor of Communication, Family Studies, and Human Development at the University of Arizona. She is the director of research for the Center for the Management of Information and site director for the National Science Foundation-sponsored Center for Identification Technology Research.

Joseph Valacich is Eller Professor of Management Information Systems at the University of Arizona. He is a fellow of the Association for Information Systems (AIS) and is a co-founder, chairman, and chief science officer of Neuro-ID, Inc. He is a prolific scholar, publishing more than 250 scholarly articles in numerous prestigious journals and conferences. 


\section{Index}

$360^{\circ}$ film $\quad 98$

$3_{3} \mathrm{D} \quad 64,101,201$

A Breathtaking Journey $\quad 15,114$

Abt, Clark 8, 29

Accuweather 171

active participation $\quad 46,223,233$

Activity Theory 201, 205

Third Generation Activity Theory (3GAT) 214

advertising 34,173

aesthetics 14, 42, 45, 96, 111. See also MDA

Model

affective appeals $\quad 95,110,114$

affordances $140,166,169,175,221,235,249$

Against All Odds $67,190,235$

agency $51,53,58,61,111,193,241$

algorithms 12, 44, 51

alterity $\quad 5^{1}$

Amnesty International $\quad 14,99$

analogue $\quad 52,146$

Ancient Greece 10

anemometer 101

Animal Crossing 35

anonymity 132,151

Another Chance 191

anthropomorphic 147

antithetical 75

appropriation $\quad 42,5^{\circ}$

archetypical 107

Aristotle 10

Artificial Intelligence $\quad 43,25^{\circ}$

attitude

attitude change $57,74,193,223,235$

Attitude Goal State 16,188

attitude scale 188

attitudinal effects $188,191,194$

counter-attitudinal 74,83

player attitude. See player

self-reported attitude testing $\quad 187$

Augmented Reality 42, 52, 97

automation

automated future $\quad 42,53$

automated system $\quad 5^{1}$

capitalistic automation 54

soft automation $45,50,5^{2}, 53$

autonomy 242,249

autonomy-supportive $149,249,25^{2}$

autotelic 50,53

avatar $14,35,112,150,172,223$

self-avatar 173

Awkward Moment 84

Azeroth 48 behavior

behavioral data. See data

behavioral tracking $\quad 5^{2}$

behavior change $73,76,123,165,172,175$,

$$
223
$$

systemic behavior 30

violent behavior $\quad 186$

believable illusion $\quad 166$

Beter in Bed 148

bias

bias blind spot $74,243,251$

bias mitigation 247,253

cognitive bias $75,240,244,251$

gender bias 79

group bias 221. See also intergroup

racial bias 222

social bias 79,81

Big Games 132

Birthday Party 191

blogs 31,131

Bogost, Ian 11, 18, 29, 6o, 64, 68, 120, 125, 129

bot 131

Breathless 98

Buffalo The Name Dropping Game 82

Burke, Kenneth 10

Buy Partisan 52

byproduct $14,121,128,133$

Cambridge Analytica $\quad 31$

Can You Fix It? 148

capitalism 53

casual revolution 8

change the world $9,37,208$

character $35,44,51,75,78,83,109,112,156$, $160,224,246,250$

ChatRoulette 149

Cicero 10

citizen participation $\quad 14,122$

climate change 16,62

cloud computing $\quad 5^{2}$

Clouds Over Sidra $\quad 98,112$

cognitive absorption $\quad 252$

cognitive load 194, 246

communication

mass communication $\quad 126$

meta-communication $\quad 215$

persuasive communication. See persuasive

risk communication 171

unidirectional communication $\quad 172$

comorbidity 142

connectedness 149,205

conspiracy theories 130

contact space $223,225,233$

Contact Hypothesis 222

Contact Space Framework $\quad$ 223, 233 


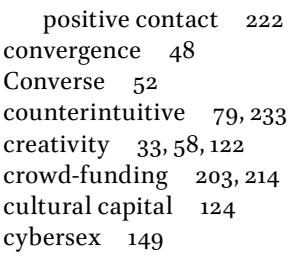

Darfur is Dying $187,192,222$ data

behavioral data 248,253

big bad data 121

big data 43,120

data-based campaign $\quad 31$

Facebook data 31

raw data 48

tracked data 47

deception-detection $\quad 242,247,253$

decision-making $\quad 242,250,253$

De la Hera, Teresa $\quad 11,15,61$

delayed revelation 83 . See also obfuscating

democracy 120,132

Depression Quest 193

design

co-design 15

design for ambiguity $143,151,160$

designing for persuasion. See persuasion

design philosophy $\quad 30,74$

emotional design $5^{0}$

Human Centered Design 190

playful design $11,42,49$

research-through-design (RtD) 95, 100, $110,113,140,143$

stealth design 73

values in design 75

Deterding, Sebastian $\quad 61,66$

dialectic 206

Die With Me $5^{2}$

digital games. See games

digital world 47

discourse

mass-media discourse 110

political discourse $\quad 31,133$

public discourse 119,133

utopian discourse 9

dispositif 11

dispositional version 243

distancing 75,85 . See also Embedded Design

Model

dynamics $13,80,166,175$. See also MDA Model dystopian 43

education $44,95,130,239$

educational games. See games

edutainment 38

playable education 38

effectiveness $\quad 222,234,239,242,249,251$

effect of controversy 190 effects of persuasive games. See persuasive games

effect studies $\quad 191$

efficiency framework 163,174

elaboration 18,194

Elaboration Likelihood Model $\quad$ 10, 194

Embedded Design Model 87

embodiment 104,110

emergence $15^{\circ}$

emotions $60,66,86,97,100,110,114,233$

emotional design. See design

emotional response 12,224

meta-emotion 194

empathy $14,62,66,106,114,223,234$

empowerment $188,204,214$

enculturation 74

engagement $31,44,54,62,96,110,120,133,167$,

$171,174,253$

visceral engagement $\quad 95,111$

Engeström, Yrjö 207, 214

enjoyment $75,83,167,173,192,194,241,253$

entertainment games. See games

entrepreneurs 37,202

ethical implications $\quad 41$

eudaimonia 194

Evokation 203, 214. See also Urgent Evoke

experience

brand experience 173

direct experience $165,167,172$

embodied experience $\quad 15,171,175$. See also embodiment

experience-taking 83

indirect experience $\quad 165,172$

transformative experience 75

visceral experience $111,164,175$

FaceApp 170

Facebook 31, 77, 209

Fair Play 222

fake news 14, 121, 134

Fatworld 35

feedback

biofeedback 111

delayed feedback $25^{\circ}$

immediate feedback $224,241,25^{\circ}$

forum 131, 204

framing devices 75,81

Friendstrap 147

functionalistic 97

game-based

game-based interventions $11,75,193$

game-based learning $44,25^{\circ}$

game-based persuasion. See persuasive games

game-based training $\quad 25^{2}$

game design $9,15,34,67,87,110,202,204,207$, 215,253

gameplay 


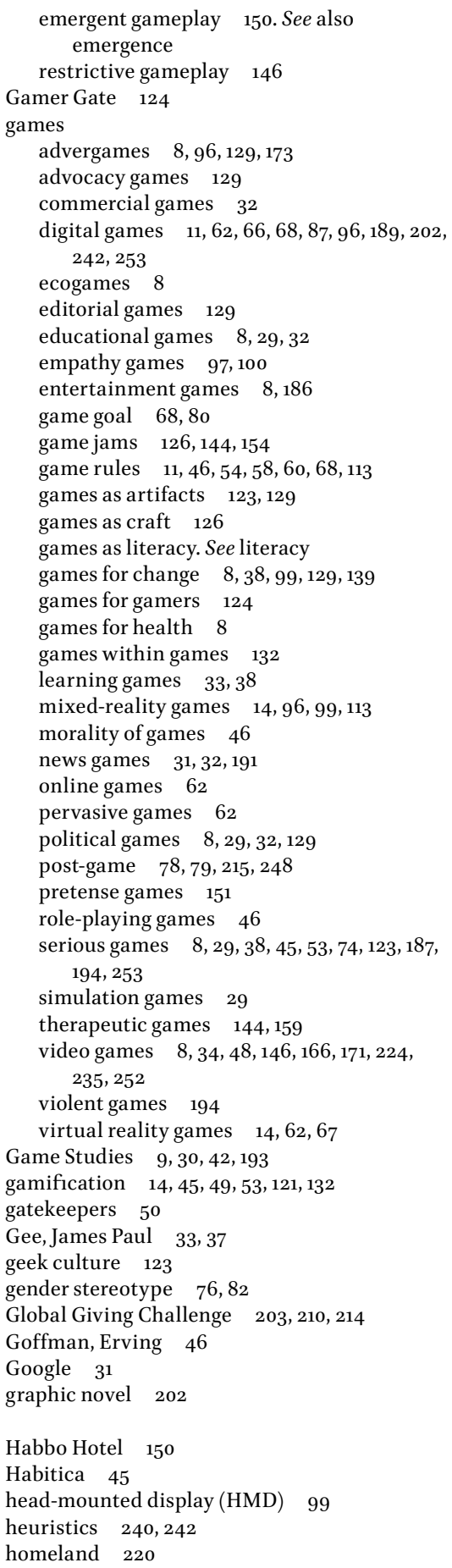

Huizinga, Johan 46, 132

Human Computer Interaction $\quad 98,110$

human rights 14, 99

Hush 99

hyper-productivity 127

Hypochondriapp $5^{2}$

hypotheticality 84

identification 193, 224. See also Contact Space Framework cognitive identification 191

identity

player identity. See player

social identity complexity 81

Social Identity Theory 221

true identity 83

IKEA Place 42

image recognition 43

imagery $10,147,166,174$

imagination $168,174,186$

immersive journalism 97

Immersive Virtual Environments $\quad$ 15, 164 . See also virtual reality

IMVU 149

information

disinformation 131

information and communication technologies 47,208

information manipulation 133

information overload 173

philosophy of information 42

sensory information $164,169,174$

Insight Heart $\quad 5^{2}$

intergroup 222, 234

intermediaries 44

intermixing 8o. See also Embedded Design

Model

internalization 13,242

Internet $123,132,149$

interpretation 205

intrinsic motivation $\quad 241,247$

involvement 171

self-involvement $\quad 224,233$. See also

Contact Space Framework

iPhone 31

Johnson, Steven $\quad 37$

Juul, Jesper 8

know-how $\quad 212$

labor $33,44,188$

division of labor $\quad 205,210$

labor displacement $\quad 44,53$

language

collective language $\quad 146$

controlling language $\quad 252$

framing language 82

individual language $\quad 146$ 
effects of persuasive games $9,18,33,59$, $163,187,194,242$

game-based persuasion $\quad 10,15,192$ micro-persuasive games 121, 129. See also micro-persuasion

persuasive game design $\quad 14,62,130$

persuasive-games theorist 37

persuasive gaming $9,18,121$

persuasiveness $11,18,191,234$

phenomenology 97

post-phenomenology 42,47 . See also re-ontologization

Plague Inc. 64

Plato 10

play

ambiguity of play 132

feel like play $\quad 42,5^{0,54}$

gender-swapping play 83

open-ended play 148

persuasive play $14,44,122,134$

play-centrism 11

play rhetoric. See rhetoric

play therapy 146

playthings $42,45,5^{1}$

purposeful play 130

repeated play 251

role-play $146,149,253$

role-playing games. See games

values at play 75

playable future $\quad 42,53$

player

active player 14

multi-player $146,25^{\circ}$

non-player $121,25^{\circ}$

player attitude $\quad 17,61,66,73,78,79,186,195$

player community $123,212,214$

player freedom $60,74,111,204,241$.

See also reactance

player identity $75,83,124$

player prior knowledge $66,68,83$

player resistance to persuasion 66,68 .

See also reactance

single-player $25^{0}$

playful apps 41

playfulness $49,52,97$

PlayStation $\quad 31$

pluralism 205

politics

political discourse. See discourse

political games. See games

politics of nutrition 35

simulated politics 35

virtualpolitik 34

Poverty is Not a Game 17,222

Power and Control $\quad 17,191$

POX Save the People 84

prejudice $74,82,224,234$

presence $15,100,114,167,171,175,224,234$.

See also Contact Space Framework pretense games. See games

priming 86

procedurality

proceduralism 11

procedural persuasion $\quad 65,68$

procedural representation $\quad 36$

procedural rhetoric $11,18,33,38,58,64$, $68,121,123,131,194$

procedural rhetoric. See procedurality

protagonist $82,96,103,110,112,234$

prototype $114,143,151$

psychological defenses 75

psychological distance 84

racist 124

reactance $74,247,249,25^{2}$

realism 105,165

refugee $14,62,67,74,107,112,192,221$

refugee asylum 220

refugee crisis 220,233

refugee journey 96,113

re-ontologization $\quad 5^{1}$

replayability 75

representation

procedural representation.

See procedurality

rule-based representation $\quad 9,57,68$

rhetoric 10

play rhetoric 119

procedural rhetoric. See procedurality

rhetorical register 33,36

verbal rhetoric 30,194

visual rhetoric 10,30

richness 224, 233. See also Contact Space

Framework

Riot Games 86

rules

experience based on rules

49

explicit rules 204

game rules. See games

rule-based representation.

See representation

social rules 150,205

safe space $80,84,203,209$

salience 250

Second Life 149

seed-money $\quad 202,214$

self concept 221. See also Social Identity

Theory

self-endorsing 173

separateness 46

serious games. See games

service

playable service $\quad 5^{1}$

self-service $\quad 45,53$

service apps 42

service sector 43

service specialist 44 


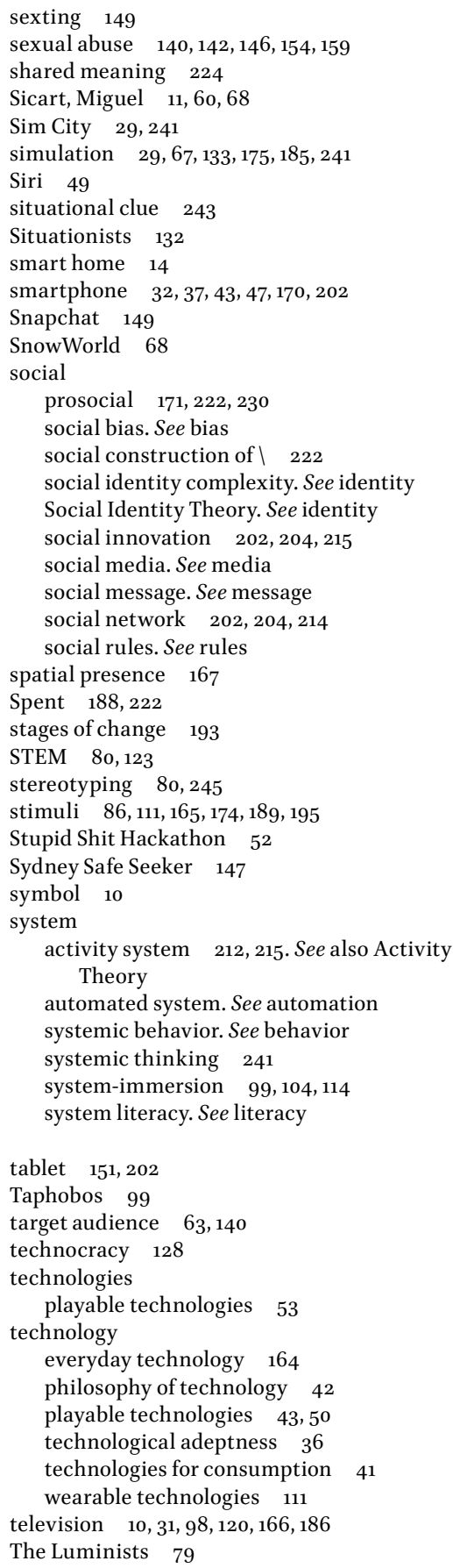

the other 224

therapeutic games. See games

The Sims Social 222

The United Nations Refugee Agency 67,220 , 226

The Wasteland 48

third object 207, 211. See also Activity Theory

This War of Mine 97

Toca Life 150

tokens 203

training $17,242,253$

transfer $202,209,215,242,248,252$

transportation 85,234

Twitter 31, 209

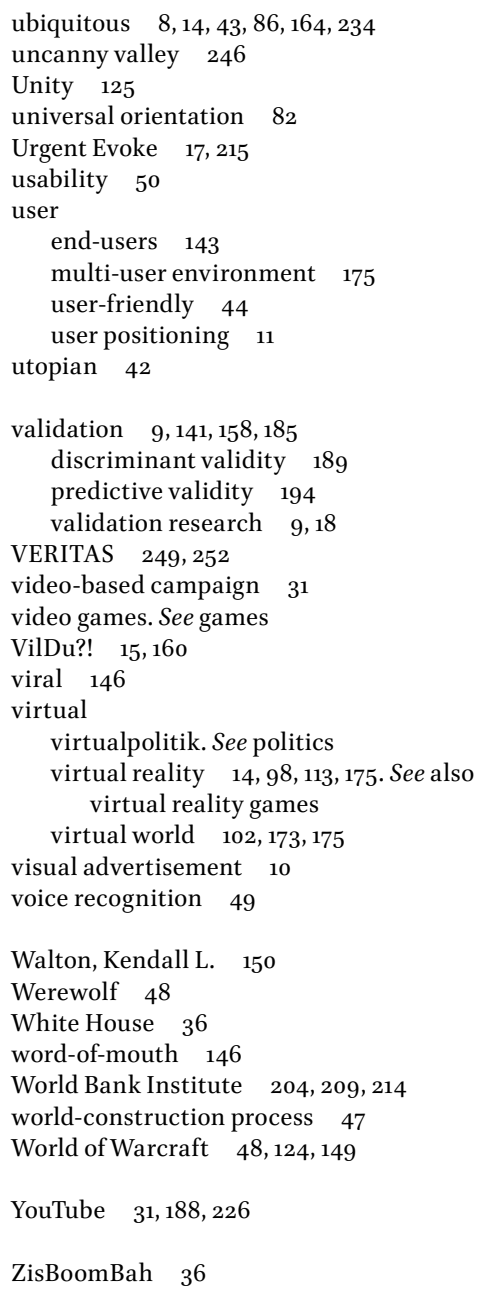


The rapid developments in new communication technologies have facilitated the popularization of digital games, which has translated into an exponential growth of the game industry in recent decades. The ubiquitous presence of digital games has resulted in an expansion of the applications of these games from mere entertainment purposes to a great variety of serious purposes. In this edited volume, we narrow the scope of attention by focusing on what game theorist Ian Bogost has called 'persuasive games', that is, gaming practices that combine the dissemination of information with attempts to engage players in particular attitudes and behaviors.

This volume offers a multifaceted reflection on persuasive gaming, that is, on the process of these particular games being played by players. The purpose is to better understand when and how digital games can be used for persuasion by further exploring persuasive games and some other kinds of persuasive playful interaction as well. The book critically integrates what has been accomplished in separate research traditions to offer a multidisciplinary approach to understanding persuasive gaming that is closely linked to developments in the industry by including the exploration of relevant case studies.

Teresa de la Hera is Assistant Professor of Persuasive Gaming at Erasmus University Rotterdam, where she is member of the Research Cluster Gaming Matters.

Jeroen Jansz is Full Professor of Communication and Media at Erasmus University Rotterdam. He holds the Chair of Communication and Media in the Department of Media \& Communication.

Joost Raessens is chair and Full Professor of Media Theory at Utrecht University, and scientific director of the Utrecht Center for Game Research (gameresearch.nl).

Ben Schouten is an artist/designer as well as a Full Professor in playful interaction at the department of industrial design of the Eindhoven University of Technology. 